\title{
Precipitation in the Waikato River Catchment
}

\author{
Stacey Maree Dravitzki
}

Meteorology

2009

\author{
A Research Project submitted to \\ the School of Geography, Environment and Earth Sciences, \\ Victoria University of Wellington, \\ as fulfilment for a PhD, 2009
}

School of Geography, Environment and Earth Sciences Victoria University of Wellington 
Dravitzki, 2009 


\section{ABSTRACT}

Observational data and numerical models were used to investigate precipitation in and around the Waikato River catchment. This economically important catchment relies on a dependable precipitation supply for agriculture and hydroelectric generation, with stations generally receiving 2,000 $\pm 300 \mathrm{~mm}$ of precipitation annually.

Long-term and inter-annual variability of total and extreme precipitation were examined using up to 100 years of observational data. Precipitation volumes within the catchment were represented by a five-day smoothed, area-averaged time series, and extreme events were defined as exceeding the 95th percentile. Atmospheric circulation oscillations correlated with the frequency of light precipitation but not with the probability of occurrence or with the magnitude of heavy precipitation events. Also no significant linear variations in precipitation (either annual totals or extreme precipitation characteristics) were found over this period, although temperature increased by $1.15 \pm 0.45^{\circ}$

A total of 63 heavy precipitation events were identified between 1996 and 2001 . An analysis of the prevailing synoptic conditions reveal that heavy precipitation was associated with the passage of cold fronts of cyclones with minima at both 500 and $1000 \mathrm{mb}$ heights. Extended periods of enhanced baroclinicity (succession of cyclones) or blocking anticyclones east of New Zealand have led to flooding in the Waikato catchment. Storm tracking showed that $10 \%$ of cyclones originating in the Tasman Sea result in heavy precipitation in the catchment.

The accuracy and value of the GFS global precipitation forecasts $\leq 180$ hours were investigated. Depending on forecast lag, the global models correctly predicted the presence of precipitation in $70-80 \%$ of forecasts, but the magnitude and distribution were often inaccurate. The probability of receiving precipitation is increased when 
more members of a lagged ensemble predict it. Forecasts with lags shorter than $\sim 96$ hours were appropriate to use as boundary constraints for mesoscale modelling.

The ability and limitations of mesoscale models to simulate the spatial distribution of precipitation were examined through high-resolution WRF simulations of three heavy precipitation events, and ten different model settings were compared for the January 2006 event. The model consistently under-predicted precipitation. The timing and location of convective precipitation, which accounted for $50 \%$ of the precipitation during two events, was physically unconstrained but regional totals were comparable to observations.

A continuous two-year numerical simulation was run to provide a precipitation climatology for data-sparse areas. The simulation gave good spatial representation of precipitation and other meteorological variables but tended to under estimate the magnitude of heavy precipitation and over-estimate light precipitation. 


\section{ACKNOWLEDGEMENTS}

This $\mathrm{PhD}$ thesis would not have been completed if I did not have the support of many people.

The most important person in this process was the primary supervisor Dr. James McGregor. Not only for the supervision and tough lessons along the way, but for organising the continual financial support. I need to thank/apologise to Dr. McGregor and Prof. Euan Smith for the reading and advice this project has required. I would also like to thank the three examiners who provided excellent feedback on this work, and my wonderful proof-readers.

This project was principally funded through an industry scholarship provided by Mighty River Power Limited. Representatives from Mighty River Power also provided guidance to the overall direction of the project and an understanding of the electricity industry in New Zealand.

Further financial support came from the Jim Ansell, and the Edward and Isobel Kidson Scholarships. I was also awarded a Postgraduate Study Abroad Award to work with Dr. Mark Falvey at the Universidad de Chile for three months.

Data and meteorological maps used in this project were provided through the National Climate Database administered through the National Institute of Water and Atmosphere, and the New Zealand Meteorological Service Ltd. Additional data was downloaded from the National Center for Environmental Prediction, the Institute for the study of the Atmosphere and Ocean, Earth System Research Laboratory.

Dr. Mark Falvey and Dr. Bethanna Jackson have provided further academic advice. Dr. Mark Henderson, Ralph Wahlirch and Jordan Wilson provided computing help. I would like to thank the other students within the school, especially my office mates, Frances and Deb, for advice, coffees, rants and friendship.

I would never have got to the end of this thesis if it were not for the continual love and support from Mike, my family, Kirsty, Clare, and Elizabeth and my other friends. You helped me retain a bit of perspective and sanity and provided many fantastic diversions. 
Dravitzki, 2009 


\section{CONTENTS}

Abstract $\quad$ iii

Acknowledgments $\quad$ iv

Table of contents $\quad$ v

List of figures $\quad$ ix

List of tables $\quad$ XV

\section{Chapters}

1 Introduction 1

1.1 Objectives of thesis . . . . . . . . . . . . . . . . . . . 1

2 Background 3

2.1 The Waikato River and catchment . . . . . . . . . . . . . . . 4

2.1.1 Weather and climate . . . . . . . . . . . . 7

2.1.2 Floods and river management . . . . . . . . . . . . . . . 8

2.2 Precipitation . . . . . . . . . . . . . . . . . . 10

3 Climate $\quad 15$

3.1 Data . . . . . . . . . . . . . . . . . . . . 16

3.2 Climate . . . . . . . . . . . . . . . . . . 17

3.3 Regional time series . . . . . . . . . . . . . . . . . . . 18

3.3 .1 Temperature . . . . . . . . . . . . . . . 18

3.3 .2 Precipitation . . . . . . . . . . . . . . . 20

3.4 Long-term variations . . . . . . . . . . . . . . . . . . . . . . 29

3.4 .1 Annual precipitation totals . . . . . . . . . . . . . . . 29

3.4 .2 Annual temperature means . . . . . . . . . . . . . . . . . 32 
3.4.3 Long-term variations in extreme precipitation . . . . . . . . . 33

3.4.4 Long-term variations in drought events . . . . . . . . . . . . 34

3.5 Climate oscillations . . . . . . . . . . . . . . . . . . 36

3.6 Discussion . . . . . . . . . . . . . . . . . . . . . . 38

4 Recent events $\quad 45$

4.1 Data . . . . . . . . . . . . . . . . . . 46

4.2 Recent extreme precipitation events . . . . . . . . . . . . 46

4.3 Case studies . . . . . . . . . . . . . . . . . . . . . . 53

4.3.1 July 1998 - a complex succession of cyclones . . . . . . . . 54

4.3.2 February 2004 - coincident ex-tropical and mid-latitude cyclone 58

4.3.3 January 2006 — subtropical and Southern Ocean cyclones . . . 60

4.3.4 Common features . . . . . . . . . . . . . . . . . . . . . 64

4.4 Storm tracks . . . . . . . . . . . . . . . . . . . 66

4.5 Discussion . . . . . . . . . . . . . . . . . . . 71

5 Global forecasts of regional precipitation $\quad 73$

5.1 Data . . . . . . . . . . . . . . . . . . . . 74

5.2 Categorical accuracy . . . . . . . . . . . . . . . . . . . 79

5.3 Time series accuracy $\ldots \ldots \ldots \ldots$. . . . . . . . . . . . . 85

5.4 Error distribution . . . . . . . . . . . . . . . . . 87

5.5 Ensemble forecasting . . . . . . . . . . . . . . . . . 91

5.5.1 Range of errors . . . . . . . . . . . . . . . 94

5.6 Probability of precipitation . . . . . . . . . . . . . 96

5.7 Discussion . . . . . . . . . . . . . . . . . . . 104

$\begin{array}{lll}6 & \text { Mesoscale simulations } & 107\end{array}$

6.1 The Weather Research and Forecasting model . . . . . . . . . . . . . . 109

6.1.1 Control model settings and parameters . . . . . . . . . . . . . . 110

6.2 Case studies . . . . . . . . . . . . . . . . . . . . . . . . . . . . 114

6.2 .1 October $2005 \ldots \ldots \ldots \ldots$

6.2 .2 January $2006 \ldots \ldots \ldots$

6.2 .3 October $2006 \ldots \ldots \ldots \ldots$

6.3 Parameter sensitivity . . . . . . . . . . . . . . . . . . . 144

6.3 .1 Topography . . . . . . . . . . . . . . . . 146

6.3 .2 Microphysics . . . . . . . . . . . . . . . 156 
6.3.3 Cumulus parameterisation ................. 162

6.3.4 Model resolution ... . . . . . . . . . . . . 165

6.4 Discussion . . . . . . . . . . . . . . . . . . . . 171

7 Dynamic downscaling $\quad \mathbf{1 7 5}$

7.1 Model set up . . . . . . . . . . . . . . . 176

7.2 Data comparison . . . . . . . . . . . . . . . . . 179

7.2.1 Daily simulation comparison . . . . . . . . . . . . . 182

7.2.2 Case study comparison . . . . . . . . . . . . . . 187

7.3 Climatology . . . . . . . . . . . . . . . . . . . 191

7.4 Subcatchment time series . . . . . . . . . . . . . . . 197

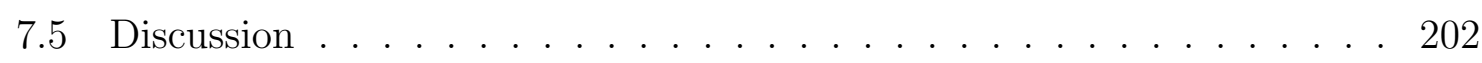

8 Conclusions 205

8.1 Climatology . . . . . . . . . . . . . . . . 206

8.2 Precipitation forecasting . . . . . . . . . . . . . . . 208

8.3 Further work . . . . . . . . . . . . . . . . . . . . . . . . . . 210

8.4 Final summary . . . . . . . . . . . . . . . . . . . . . . . 212

$\begin{array}{ll}\text { References } & 213\end{array}$

\section{Appendices}

$\begin{array}{ll}\text { A Climate stations } & 223\end{array}$

$\begin{array}{ll}\text { B Statistics } & 231\end{array}$

B.1 Event definitions . . . . . . . . . . . . . . . . . . . . . 231

B.2 Trend calculation (including confidence calculation) . . . . . . . . 231

B.3 Cluster analysis . . . . . . . . . . . . . . . . 232

B.4 Forecast verification . . . . . . . . . . . . . . . . . 233

B.4.1 Definitions . . . . . . . . . . . . . . . 233

B.4.2 Contingency tables . . . . . . . . . . . . . . 233

B.4.3 Time series validation . . . . . . . . . . . . . . . 234

$\begin{array}{ll}\text { C WRF control files } & 237\end{array}$ 


\section{FIGURES}

2.1 Southwest Pacific map . . . . . . . . . . . . . . . . . . 4

2.2 The central North Island of New Zealand . . . . . . . . . . . . . . . 5

2.3 Vertical profile of the Waikato River hydroelectric scheme . . . . . . . . 6

2.4 Spatial and temporal variability of precipitation . . . . . . . . . . . 10

3.1 The location of rain gauges explicitly mentioned in Chapter $3 \ldots 17$

3.2 Mean total annual precipitation map . . . . . . . . . . . . . . . . 18

3.3 Mean temperature map . . . . . . . . . . . . . . . . . . . . . . . . . . . 19

3.4 The stations that met the completeness criteria to be included in the regional time series . . . . . . . . . . . . . . . . 21

3.5 Variations in daily precipitation observed around the Waikato River catchment . . . . . . . . . . . . . . . 2 23

3.6 Smoothing daily regional precipitation time series . . . . . . . . . . . 24

3.7 The regional precipitation time series . . . . . . . . . . . . . 25

3.8 Distribution of daily precipitation totals . . . . . . . . . . . . . 26

3.9 Regional total annual precipitation trend . . . . . . . . . . . . . . 30

3.10 Total annual precipitation trends for selected rain gauges . . . . . . . . 31

3.11 Mean annual temperature time series . . . . . . . . . . . . . . . . 32

3.12 The occurrence frequency of extreme events . . . . . . . . . . . . 33

3.13 Changes in event characteristics . . . . . . . . . . . . . . . . . . 34

3.14 The frequency of drought occurrence . . . . . . . . . . . . . . . . 35

3.15 Changes in drought characteristics . . . . . . . . . . . . . . . . . 35

3.16 Comparing large-scale circulation indices to the magnitude of extreme

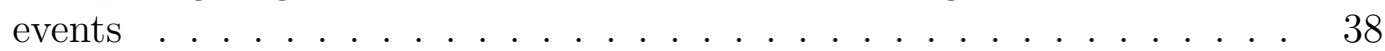

3.17 Comparing large-scale circulation indices values during extreme events . $\quad 39$

3.18 Probability relationships between extreme event occurrence and largescale circulation indices . . . . . . . . . . . . . . . . . . . 40

4.1 Seasonal characteristics of extreme precipitation events . . . . . . . . 47

4.2 'Origin' classification boundaries for features . . . . . . . . . . . . . . . 49 
4.3 The regional area-averaged time series for the event of July 1998 . . . . 54

4.4 MetService analysis maps for the event of July 1998 . . . . . . . . . . 55

4.5 Geopotential height maps for the event of July 1998 . . . . . . . . . . 56

4.6 Daily precipitation distribution maps for the event of July 1998 . . . . 57

4.7 The regional area-averaged time series for the event of February 2004 • 58

4.8 MetService analysis maps for the event of February 2004 . . . . . . . . 59

4.9 Geopotential height maps for the event of February 2004 . . . . . . . . 60

4.10 Daily precipitation distribution maps for the event of February 2004 . . 61

4.11 The regional area-averaged time series for the event of January 2006 . . 62

4.12 Geopotential height maps for the event of January 2006 . . . . . . . . . 62

4.13 Daily precipitation distribution maps for the event of January 2006 . . 63

4.14 Storm tracks coinciding with heavy precipitation . . . . . . . . . . 69

4.15 Storm track frequency . . . . . . . . . . . . . . . . . . . . 70

5.1 Locations of hourly observations and GFS grid point data . . . . . . 75

5.2 Interpolation of data sets for comparison . . . . . . . . . . . . 78

5.3 Value of area averaging . . . . . . . . . . . . . . . 80

5.4 Categorical hit rate and bias . . . . . . . . . . . . . . . 82

5.5 Categorical error analysis . . . . . . . . . . . . . . . . 83

5.6 Time series error analysis . . . . . . . . . . . . . . 86

5.7 Distribution of forecast errors _ . . . . . . . . . . . . . . . . . . 88

5.8 Statistical accuracy measures for individual stations . . . . . . . . . . . 89

5.9 Statistical accuracy measures for individual stations . . . . . . . . . . . 90

5.10 Complete precipitation runs issued for the month of January 2006 . . . 92

5.11 Ensemble forecast charts valid at 1800 UTC January 24, 2006 . . . . . 93

5.12 Mean and spread of precipitation forecasts at same valid time . . . . . 94

5.13 The spread of model runs correctly identifying precipitation _ . . . . . 95

5.14 Probability distribution functions for the 48-hour lag forecast . . . . . . 98

5.15 Subsequent forecast conditional probability functions . . . . . . . . . . 99

$5.16 \log$ scale of Figure $5.15 \ldots \ldots$

5.17 Precipitation observation conditional probability functions . . . . . . . 101

5.18 Log scale of Figure 5.17 . . . . . . . . . . . . . . . . 102

5.19 Comparison of conditional probability functions for specified precipitation amounts . . . . . . . . . . . . . . . 103

5.20 Conditional probability of exceeding a threshold depending on frequency of previous exceedence . . . . . . . . . . . . . . . . . . 104 
6.1 Outer domain for high-resolution simulations . . . . . . . . . . . . . . . 112

6.2 Middle domain for high-resolution simulations . . . . . . . . . . . . . . 112

6.3 Inner domain for high-resolution simulations . . . . . . . . . . . . . . . 113

6.4 Vertical sigma levels for high-resolution simulations . . . . . . . . . . . 114

6.5 Data locations mentioned in this chapter . . . . . . . . . . . . . 116

6.6 Daily precipitation time series for the October 2005 event . . . . . . . . 117

6.7 MetService analysis maps for the October 2005 event . . . . . . . . . . 118

6.8 Satellite images for the October 2005 event . . . . . . . . . . . . . . . . 119

6.9 Hourly observations for the October 2005 event . . . . . . . . . . . 120

6.10 GFS predictions for the October 2005 event . . . . . . . . . . . . . 122

6.11 WRF simulation of the October 2005 event . . . . . . . . . . . . . . 123

6.12 Accumulated precipitation over the October 2005 event . . . . . . . . 125

6.13 Hourly modelled precipitation time series for the October 2005 event . 126

6.14 Daily precipitation time series for the January 2006 event . . . . . . . . 127

6.15 MetService analysis maps for the January 2006 event . . . . . . . . . . 128

6.16 Satellite images for the January 2006 event . . . . . . . . . . . . . . 129

6.17 Hourly observations for the January 2006 event . . . . . . . . . . . . . 130

6.18 GFS predictions for the January 2006 event . . . . . . . . . . . . . . 131

6.19 WRF simulation of the January 2006 event . . . . . . . . . . . . . . . 132

6.20 Accumulated precipitation over the January 2006 event . . . . . . . . . 133

6.21 Hourly modelled precipitation time series for the January 2006 event . 134

6.22 Daily precipitation time series for the October 2006 event . . . . . . . 136

6.23 MetService analysis maps for the October 2006 event . . . . . . . . . 137

6.24 Satellite images for the October 2006 event . . . . . . . . . . . . . . . . 138

6.25 Hourly observations for the October 2006 event . . . . . . . . . . . . 139

6.26 GFS predictions for the October 2006 event . . . . . . . . . . . . . 140

6.27 WRF simulation of the October 2006 event . . . . . . . . . . . . . . . 141

6.28 Accumulated precipitation over the October 2006 event . . . . . . . . . 142

6.29 Hourly modelled precipitation time series for the October 2006 event . 143

6.30 Time series comparison for each of the topographic simulations . . . . . 147

6.31 The hourly time series rmse for the topographic simulations . . . . . . 149

6.32 Six-hour precipitation accumulation during the frontal period for the topographical simulations . . . . . . . . . . . . . . . . 150

6.33 Twenty-four-hour precipitation accumulation during the convective period for the topographical simulations . . . . . . . . . . . . . . 151 
6.34 Settings for the vertical precipitation profiles.

6.35 Vertical cross-section (cyan in Figure 6.34) of q_rate and relative wind speeds at 0300 UTC 24 January 2006.

6.36 Vertical cross-section (green in Figure 6.34) of q_rate and relative wind speeds at 0600 UTC 25 January 2006.

6.37 Time series comparison for each of the microphysics simulations . . . 157

6.38 The rmse for individual hourly precipitation over the event for each of the four microphysics schemes . . . . . . . . . . . . . . .

6.39 Six-hour precipitation accumulation during the frontal period for the microphysics simulations . . . . . . . . . . . . . . 160

6.40 Twenty-four-hour precipitation accumulation during the convective period for the microphysics simulations . . . . . . . . . . . . . 161

6.41 Time series comparison for each of the cumulus simulation . . . . . . 163

6.42 The rmse for individual hourly precipitation over the event for the $\mathrm{Cu}-$ mulus simulation . . . . . . . . . . . . . . . . . 164

6.43 Twenty-four-hour precipitation accumulation during the convective period for the cumulus simulations . . . . . . . . . . . . . . . . 164

6.44 Time series comparison for each of the domain resolution simulations . 167

6.45 The rmse for individual hourly precipitation over the event for each of the domain resolution simulations . . . . . . . . . . . . . . . . 168

6.46 Six-hour precipitation accumulation during the frontal period for the domain resolution simulations . . . . . . . . . . . . . .

6.47 Twenty-four-hour precipitation accumulation during the convective period for the domain resolution simulations . . . . . . . . . . . 170

7.1 Domain boundaries for the WRF dynamic downscaling simulation. . . 178

7.2 Grid-squares representing each subcatchment . . . . . . . . . . . . . . . 179

7.3 Comparison of the 2006 total accumulated precipitation for New Zealand 180

7.4 Comparison of the 2006 total accumulated precipitation for the Waikato River catchment . . . . . . . . . . . . . . . . . . . . 181

7.5 Mean error of downscaled data in New Zealand . . . . . . . . . . . . . 183

7.6 Mean error of downscaled data in the Waikato River catchment . . . . 184

7.7 Rmse of downscaled data in New Zealand . . . . . . . . . . . . . . . . . 185

7.8 Rmse of downscaled data in the Waikato River catchment . . . . . . 186

7.9 Mean error vs. rmse at New Zealand rain gauge locations . . . . . . . . 186

7.10 Mean absolute percentage error of downscaled data in New Zealand . . 187

7.11 Mean absolute percentage error of downscaled data in the Waikato River catchment . . . . . . . . . . . . . . . . . . . 188 
7.12 Hourly precipitation time series for the October 2005 event . . . . . . . 189

7.13 Hourly precipitation time series for the January 2006 event . . . . . . . 190

7.14 Hourly precipitation time series for the October 2006 event . . . . . . . 191

7.15 Average total annual precipitation in New Zealand . . . . . . . . . . . 193

7.16 Average total annual precipitation in the Waikato River catchment . . 194

7.17 Average total summer precipitation in the Waikato River catchment . . 195

7.18 Average total autumn precipitation in the Waikato River catchment . . 195

7.19 Average total winter precipitation in the Waikato River catchment . . . 196

7.20 Average total spring precipitation in the Waikato River catchment . . . 197

7.21 Simulated daily precipitation time series in each subcatchment . . . . . 198

7.22 Histogram of daily precipitation values in each subcatchment . . . . . . 200 
xvi

Dravitzki, 2009 


\section{TABLES}

4.1 The 63 extreme precipitation events between 1996 and 2007 . . . . . 50

4.2 Summary of features observed in extreme events . . . . . . . . . . . 52

5.1 Precipitation categories and their natural occurrence probabilities . . 81

5.2 Full categorical contingency table example for 6-hour lag forecast . . . 81

5.3 Contingency table example for six-hour lag forecast being over $20 \mathrm{~mm}$. 84

6.1 The WRF settings for each high-resolution Control run simulation . 111

6.2 Alternative high-resolution WRF simulations of January 2006 event . . 145

6.3 Precipitation summary during each event . . . . . . . . . . . . . . 146

6.4 Domain details for model resolution simulations . . . . . . . . . . . 165

7.1 The WRF settings for the two-year dynamic downscaling simulation. 177

7.2 The total average volume of precipitation in each subcatchment . . . . 201

B.1 Contingency table format . . . . . . . . . . . . 233 
CHAPTER 1

\section{INTRODUCTION}

This research investigates the precipitation climatology and models precipitation characteristics of the Waikato River catchment, with a focus on past extreme events. The Waikato River catchment is an economically important region of New Zealand where the primary industries, agriculture and hydroelectric power generation, are dependant on regular precipitation but are adversely affected by the precipitation extremities of droughts and flooding.

\subsection{Objectives of thesis}

The wider aim of this project is to provide real-time forecasts about the natural inflows of the Waikato River catchment on a scale of days to weeks. This study of the precipitation is the first part of a larger project aimed at predicting precipitation and river inflows for the hydroelectric power generator. Other researchers are investigating the optimisation of numerical weather prediction models and developing the required hydrological models. My contribution to this project is an evaluation of the current climatological and meteorological conditions during extreme events and the predictive abilities of the meteorological models, thereby estimating the uncertainties of the precipitation forecasts, and providing a climatological context of precipitation amounts.

This aim is broken down into five objectives:

1. To complete a precipitation climatology for the Waikato River catchment in order to provide context of precipitation amounts and to identify any long-term 
precipitation trends (particularly extreme precipitation events) within this area;

2. To analyse the characteristics of synoptic weather scenarios that have produced heavy precipitation in the Waikato River catchment, especially those that led to flooding, and to identify which weather characteristics are likely to produce heavy precipitation in the future;

3. To determine the accuracy and stability of the global forecasts at predicting precipitation within this catchment to determine the model's value as an extended forecast and boundary constraint for mesoscale modelling;

4. To simulate previous heavy precipitation events using a mesoscale model to illustrate the improvement in forecasting precipitation distribution and to examine the mechanisms of precipitation generation in the model, which determines the model's abilities and limitations of forecasting future heavy precipitation events; and

5. To use dynamic downscaling techniques to provide a model climatology in datasparse sub-catchments and validate the mesoscale model's ability to predict precipitation over the complete range of weather systems affecting the area.

To cover these objectives the thesis has been split into six chapters: background; climate; recent events; global forecasts of regional precipitation; mesoscale simulations; and dynamic downscaling. The appropriate literature is discussed within each of these chapters. 
CHAPTER 2

\section{BACKGROUND}

Given the importance of water to life, the idea of predicting where precipitation will occur has been the principal idea of many investigations over hundreds of years. Trying to predict the precipitation within a specific catchment was not a novel idea in the 1920s when Day (1926) investigated a Canadian basin. The complexity of the hydrological cycle means that modern research is split into multiple disciplines: weather, climate, hydrology, ground water, and oceanography. The exact movement and distribution of water molecules is influenced by the surrounding environment (e.g. other water molecules, topography, vegetation, etc) at all stages in the hydrological cycle. This project only addresses the weather and climatological aspects of the hydrological cycle within the Waikato River catchment.

The Waikato River is economically important to New Zealand because of the strategic hydroelectric development and agricultural activities within the catchment area. Both of these industries have high dependence on the available water resource. Despite the long history of precipitation research on a catchment scale, continual research is required due to the continual developments in computer resources, analysis techniques, the inherent difficulties in dealing with precipitation and the imperfect simulation capabilities of numerical weather models.

The Waikato River is located in the central North Island of New Zealand (the catchment boundary and places mentioned in this thesis are shown in Figures 2.1 and 2.2). The following description of the river and catchment are summarised from Roper (2001) and Ridall (1967). 


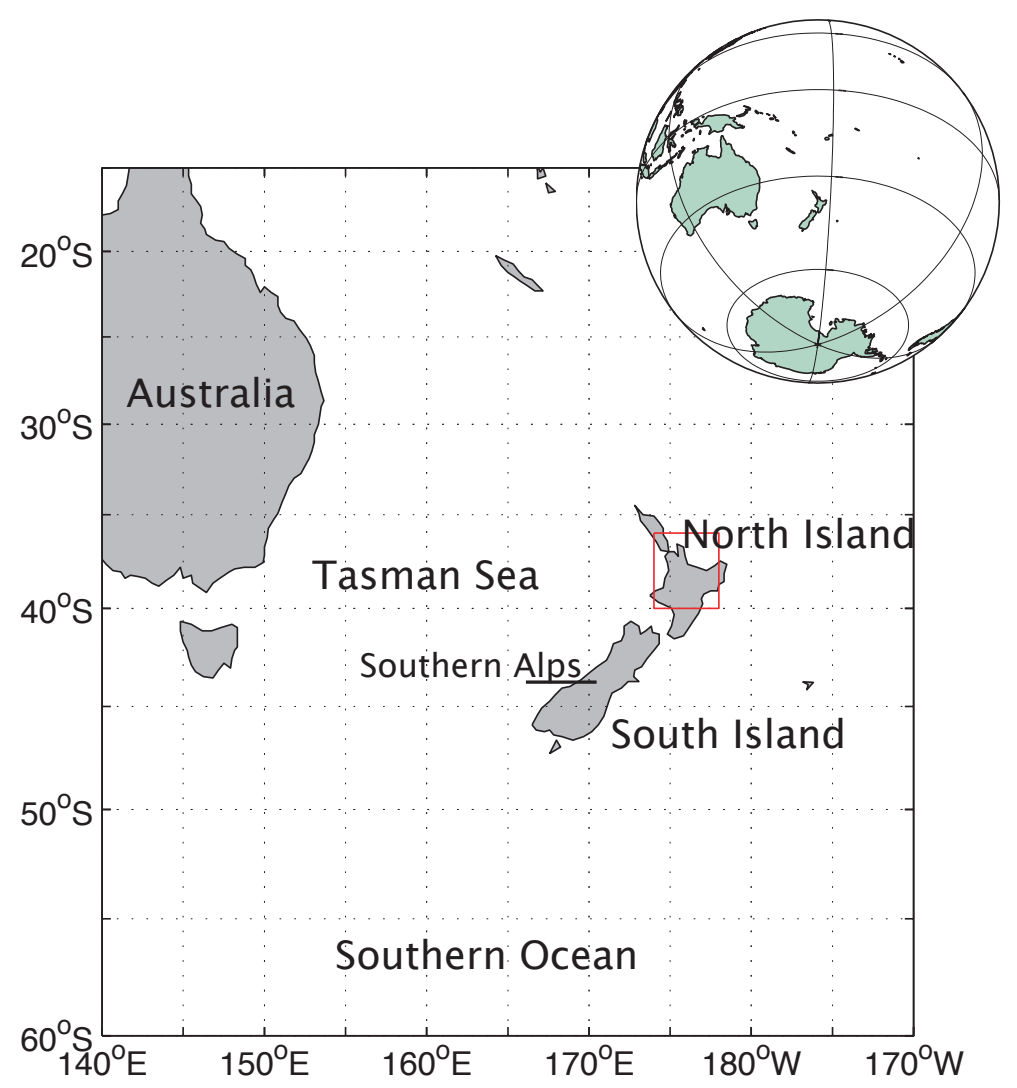

Figure 2.1 Southwest Pacific map showing the general location of the Waikato River catchment and identifying the locations of places named within this thesis. An enlargement of the central North Island (bounded by the red box) is provided in Figure 2.2.

This chapter provides a brief background to: the Waikato River and catchment, the climate and weather, floods and management, and the inherent difficulties in dealing with precipitation.

\subsection{The Waikato River and catchment}

The Waikato River is New Zealand's longest river $(425 \mathrm{~km})$, forming the centrepiece of the Waikato region. The head of the river is on the northeastern slopes of MtR̃uapehu (2797 $\mathrm{m}$ asl), but the river loses its identity to the Tongariro River for the $42 \mathrm{~km}$ leading into Lake Taupo. Lake Taupo is the largest lake in Australasia (surface area of $611 \mathrm{~km}^{2}$ and volume of $59 \mathrm{~km}^{3}$ ) and contains $93 \%$ of the available water storage area for the hydroelectric scheme (Figure 2.2). In this upper part of the catchment water is also diverted from other rivers through the Tongariro Power Development on the Tongariro section of the Waikato River.

From the control gates at Lake Taupo, the river flows $160 \mathrm{~km}$ northward through 


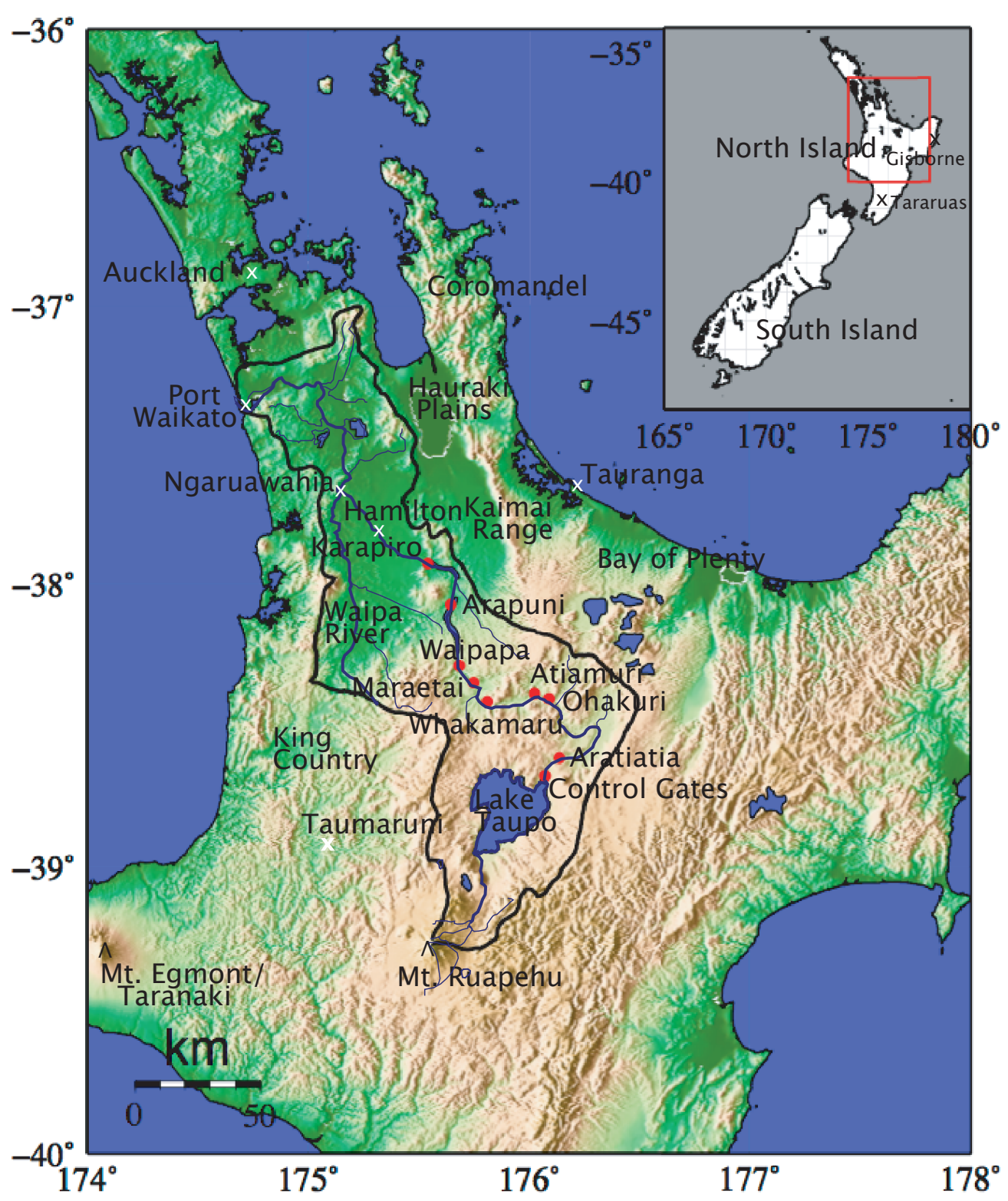

Figure 2.2 An enlargement of the central North Island of New Zealand identifying named locations on a digital elevation map representing the topography of this region. The solid black line shows the catchment boundary of the Waikato River; the Waikato River and major tributaries are shown in dark blue; Volcanic peaks are identified by triangles, towns and cities by white crosses and hydropower dams by red circles. Other names refer to the general region.

a series of eight hydroelectric dams and dropping a total $330 \mathrm{~m}$ in altitude (the river profile is shown in Figure 2.3). When water initially leaves the lake, it flows in a wide shallow channel through a mainly pumice catchment largely developed for forestry and grazing to Lake Ohakuri. Between Ohakuri and Karapiro, the river flows in a deeply 


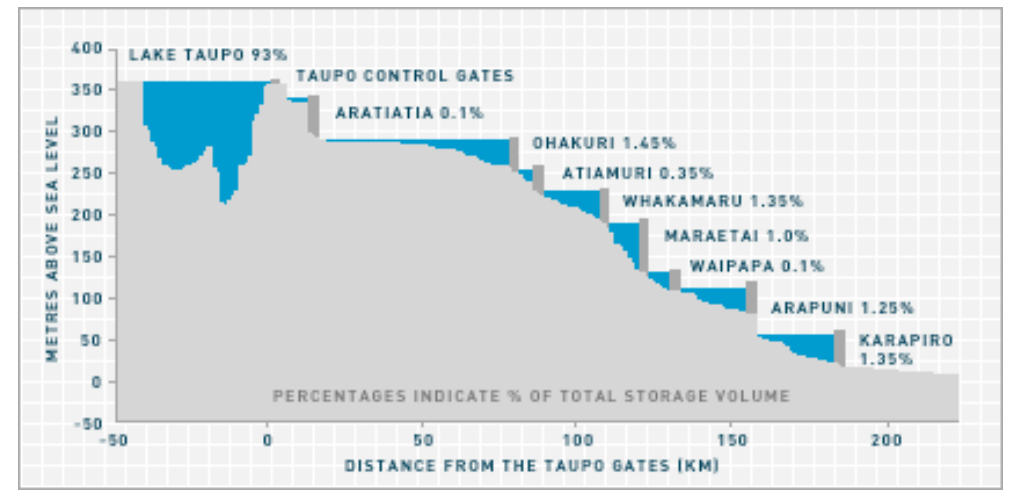

Figure 2.3 A vertically exaggerated profile of the Waikato River hydroelectric scheme showing storage capacities. This figure is taken from Roper (2001).

incised channel that is now a series of hydro-lakes. Thirty-five percent of the water reaching Karapiro is contributed by tributaries downstream of the Taupo control gates and cannot be stored within the system. Of the five big tributaries that flow in, four have catchments under intensive agriculture.

Downstream of the hydroelectric scheme, the river flows over flat, often swampy, lowlands and is joined by its major tributary, the Waipa River, at Ngaruawahia. The Waipa River has a catchment of $3,050 \mathrm{~km}^{2}$ and begins in the King Country over $160 \tilde{\mathrm{km}}$ from Ngaruawahia. The Waikato River finally discharges into the Tasman Sea at Port Waikato, approximately $30 \mathrm{~km}$ south of Auckland. Overall it drains water from $14,258 \mathrm{~km}^{2}$ of the North Island (Roper, 2001, size varies in other publications), in a predominantly unstable channel with highly variable flows.

Lake Taupo was created around 26,500 years ago by the Oruanui eruption (Roper, 2001, and references within). Volcanic sediment builds up in the river and washes downstream during high flows forcing the river to change course. The Waikato River has changed its course between the Hauraki plains and the Hamilton basin at least four times during the past 220,000 years, but has been on its present course through the Hamilton basin for the last 15,000 years.

The geology of the Taupo/Waikato catchment has a significant influence on rainfall runoff and river flows. Porous pumice, volcanic ash and ignimbrite areas absorb much of the rain that falls on them forming groundwater, which leads to steady, spring-fed base 
flows. Only about $10 \%$ of the water flows off as flood run-off during very intense rain (Selby, 1967). In contrast, the impermeable sedimentary rock of the Waipa catchment results in rapid surface run-off and frequent flash floods.

\subsubsection{Weather and climate}

Weather patterns in New Zealand are typical of oceanic mid-latitude locations and are strongly influenced by the southern hemisphere westerly wind belt. Precipitation at these latitudes principally occurs in troughs and frontal type mid-latitude cyclones (Sturman and Tapper, 2005; Türkes, 1996), although convective systems also produce some of the precipitation. The distribution of precipitation in New Zealand is significantly altered by orographic enhancement (Garnier, 1950).

The Tasman Sea to the west of New Zealand has been identified as a region of high cyclogenesis in previous hemispheric-scale tracking studies (Sinclair (1995a), (1995b), and (1994), and Trenberth (1991)). Cyclonic weather features pass over New Zealand from the west at an average interval of one every six-seven days (de Lisle, 1967). Additionally, on average one ex-tropical cyclone (Sinclair, 2002) and two to three subtropical cyclones (Schröder, 2009) hit New Zealand every year. Heavy precipitation and flooding in the Waikato River catchment has been previously associated with all types of cyclones (Roper, 2001).

The Waikato River catchment is sheltered to the northeast by the Kaimai and Coromandel Ranges (highest peak at 952 masl); to the south by the central North Island plateau and volcanoes (Mt Ruapehu at 2,797 masl); and along the western coast by the King Country (peak at 756 masl). These topographic features can protect the catchment during severe precipitation but also prevent smaller storms reaching the catchment during droughts.

Settled weather is associated with the passage of anticyclones across the North Island. In summer, anticyclonic conditions may in extreme cases persist for up to four weeks (de Lisle, 1967). The heaviest rainfalls are produced by 'depressions' moving 
over the area from the north or northwest and are associated with relatively warm moist air (de Lisle, 1967). The terms depression, low and mid-latitude cyclone all refer to the same phenomena and the last term is used for work in this thesis.

The regional climate is described as temperate with warm, humid summers and mild winters. Precipitation has a winter maximum with larger totals recorded at higher altitude rain gauges. Precipitation is generally adequate for agricultural and pastoral purposes. Temperature variations are dampened by the close proximity of ocean and diurnal variations are generally larger than seasonal variations. Further details about the general climate can be found in Garnier (1950) and more recently in Salinger et al. (2001); (1995); Brenstrum (1998); and Basher and Thompson (1996).

\subsubsection{Floods and river management}

The flooding of a large river such as the Waikato can have significant consequences which are exacerbated by the high level of development in the catchment, especially on the flood plains, and an increasing population density along the riverbanks. Floods in the past century have caused damage and losses to property, farmland, stock, farm production, roads, bridges, railways and stop banks, as well as causing general disruption to farmers and local communities (Roper, 2001).

The hydroelectric scheme on the Waikato River produces approximately $13 \%$ of NewZ̃ealand's current electricity requirements (4,400 GWh annually). It also provides frequency control and can meet sudden changes in demand or respond to failures of other power stations or transmission circuits. Mighty River Power (a State-owned enterprise) operates eight hydroelectric power stations along the length of the river. The company also controls the floodgates at the northern outlet of Lake Taupo that feed the Waikato River. Once water is released from Lake Taupo it takes approximately 18 hours to reach the eighth power station.

The resource consent* for the management of the river sets a number of require-

*A legal contract between the operators and the government dictating the terms of use of the river, Mighty River Power Ltd. resource consent is included as reference: Roper (2001) 
ments, e.g. a minimum flow of $148 \mathrm{~m}^{3} / \mathrm{s}$ must be maintained below Lake Karapiro (Roper, 2001). All lakes also have an allowable operating range and maximum flood level that can only be exceeded once every 100 years. For example, the maximum operating height of Lake Taupo is $357.387 \mathrm{~m}$ asl; because the lake also has a minimum level (355.85 $\mathrm{m}$ asl) the live storage (maximum amount of water available for generation) is restricted to 800 million $\mathrm{m}^{3}$ ( $\approx 1 \%$ of Lake Taupo's total volume). Storage on the other hydro-lakes is restricted to 61 million $\mathrm{m}^{3}$.

During extremely high flow events, the regional council can invoke an additional set of 'flood mitigation' rules. These are principally intended to prevent the catastrophic failure of a hydro-dam, which would cause cascading failures down river. However, this would require exceptionally large flows that are unlikely to occur in the current climate. The flood rules are used predominantly to minimise damage along the length of the river. Flooding most frequently occurs on the flood plains near the mouth of the river, below the confluence with the uncontrolled Waipa River, which is prone to flash flooding. By restricting the outflow at Karapiro dam the maximum flood peak on the flood plains is reduced, but restricting the flow at Karapiro may cause flooding upstream as both catchments regularly flood together. As there is limited storage in the small hydro-lakes along the river the mitigation options are significantly increased if water can be contained in Lake Taupo, or flushed through the system prior to the event.

Flood analysis conducted for the resource consent showed that $67 \%$ of annual maximum floods occur in the winter-spring period (July-October), and only $14 \%$ of annual maximum floods have occurred in the summer months. However, the summer floods have included three of the largest recorded floods: in 1907, 1958 and 2004 (this last post the consent process). Since the river has been controlled there has been less natural variability in river/lake levels and flood peaks, but the flood plains are now more extensively used for residential and farming activities. 


\subsection{Precipitation}

Precipitation is any water that leaves the atmosphere. Although it is predominantly liquid water (rain) in the Waikato, it also includes snow, hail and ice. "Precipitation" is a more inclusive term than rainfall, and since rain gauge observations cannot distinguish between these phases the term precipitation is used throughout this thesis. Precipitation is important because it has such a large impact on our lives, providing water required to grow food and produce electricity, less positively producing devastating floods and drought. Although these are the extremes, the possibility of precipitation is the aspect of a weather forecast that is most likely to make us change our daily plans.

Precipitation may be the most influential, but also the most difficult, meteorological parameter to constrain because of its high spatial and temporal variability. Most people living in New Zealand have been caught by surprise at least once by the sudden appearance of rain, apparently from out of nowhere, or have seen a line across a road where one side is dry and the other wet (anecdotal).

Figure 2.4 shows two radar images taken one hour apart by the Auckland Radar at hours 1900 and $2000 \mathrm{UTC}^{\dagger}$ on 17 July 2008. Radar measurements can be used as a

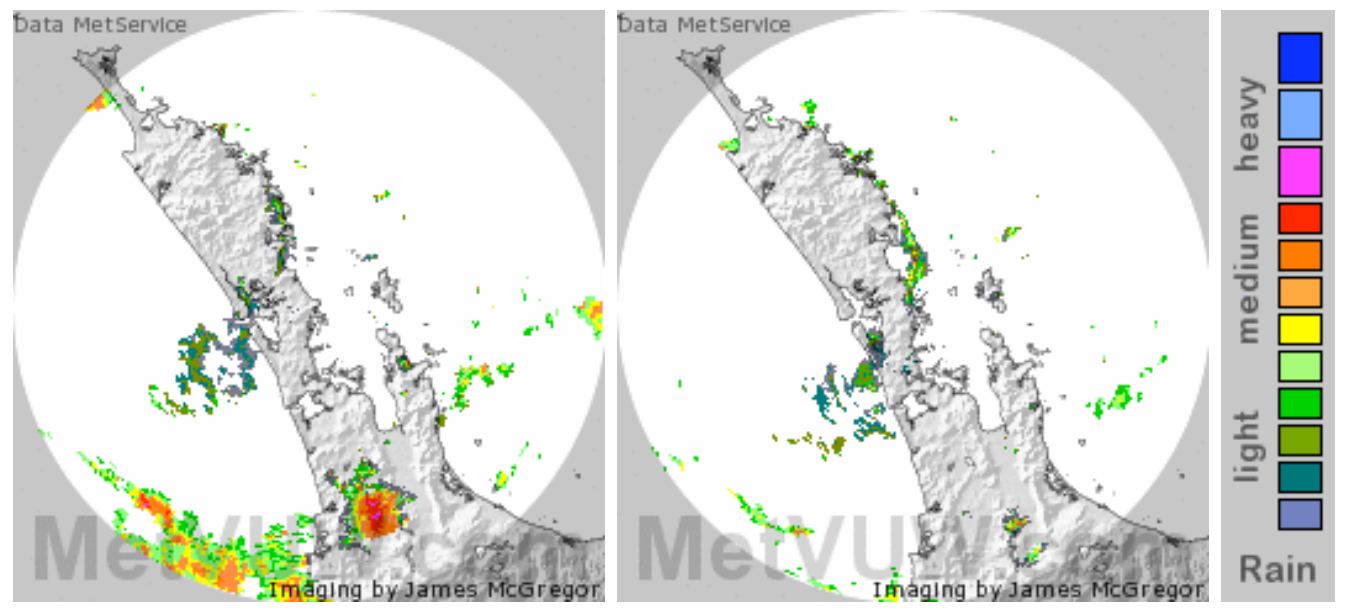

Figure 2.4 Example radar images showing the spatial and temporal variability of precipitation; (a) precipitation at 1900 UTC on 17 July 2008, and (b) one hour later. Note: in all figures subplots are numbered alphabetically from left to right, top to bottom.

\footnotetext{
${ }^{\dagger}$ All times and dates throughout the thesis are in UTC time unless otherwise stated.
} 
proxy for instantaneous precipitation fields. Some of the variation is simply due to the rain band shifting southeast with the general circulation pattern at the time; however, the shape of precipitation cells has also changed and the heavy precipitation cell to the south in the image has almost completely disappeared. This example was taken at random, but almost any hour-long period on a wet day could also show substantial variation in the instantaneous precipitation field.

The variability in precipitation is due to the different mechanisms that form precipitation in the atmosphere. Air masses interact with each other and the surface as they move through the atmosphere (Holton, 2004) producing cyclonic lifting, convection, or convergence (Sturman and Tapper, 2005) that can all lead to precipitation. Individual raindrops (with a minimum radius size of 100 micrometers) form and interact on a micrometer scale, but entire air masses can interact producing precipitation on a scale of a hundred metres (e.g. convective storms) to thousands of kilometres (e.g. cyclones) (Holton, 2004).

This natural variability means that the precipitation at one location cannot always be reliably inferred from a nearby observation. It is physically impossible to continuously measure precipitation everywhere, although radar can provide a proxy for the instantaneous precipitation field within its field of view. Point measurements are routinely made, often accumulating precipitation over a time period to represent the precipitation over an area. When records began in New Zealand in 1860 (Penny, 2003) these were 24-hour accumulations at manual rain gauges. Rain gauges were also located in places that were easy to access, so distribution was irregularly spaced, and primarily restricted to populated places.

Currently most rain gauges in New Zealand are the automatic tipping bucket type that record the time taken to collect each $0.1 \mathrm{~mm}$ of precipitation, but these instruments are not perfect. Although a number of quality controls are performed before the data are entered into the New Zealand Climate Database (Penny, 2003), there is still no guarantee that the data are correct. There is always the possibility that: an animal 
has altered the water level (i.e. drunk from the gauge), the instrument malfunctioned, the top of the gauge iced over, precipitation evaporated from the gauge before it was counted, or windy conditions led to gauge under-catch. Radar is more frequently being used to measure the instantaneous precipitation field and can be used to approximate precipitation amounts. Unfortunately the Auckland Radar only covered the northern section of the Waikato River catchment during the collection periods. With the addition of the New Plymouth Radar in 2008 a composite image could now be obtained for the whole catchment. Continuous research in the improvement of collection and quality of observational precipitation records is conducted due to these error sources.

A similar issue is encountered with precipitation modelling. The spatial and temporal resolution of the model is insufficient to represent all the scales of precipitation variability. The unpredictability of precipitation is accentuated by various sources of error including representation of orography and land use, physical processes in clouds, model initialisation errors of various fields (particularly water substance quantities) and spatial resolution (Grubišić et al., 2005). The quantitative precipitation forecast (QPF) skill is low in operation forecasting, and improvements are slow compared to other variables. The importance of improving QPF has long been recognised by the meteorological modelling community (Grubišić et al., 2005, and papers within) due to the low skill of the models and high social-economic importance of precipitation.

It is this small-scale fractal-like variability of precipitation (Lovejoy and Schertzer, 2006) that drives meteorologists to continually increase the resolution of measurements and models (both spatially and temporally). These variations are not systematic, so unlike temperature, pressure and other atmospheric variables, precipitation cannot be easily interpolated between locations. Many of the statistical methods used to interpolate atmospheric variables are based on Gaussian distributions of the data; however precipitation in general is closer to gamma distribution (Wilks, 1995). Gamma, lognormal or square root distributions are used for precipitation analysis e.g. You et al. (2007) or Lovejoy et al. (1987). 
The gamma distribution of precipitation is one of the reasons that statistical interpolation (averaging, downscaling) of precipitation is so difficult. In order to investigate trends in precipitation variation over periods of time, a single station is often used. However, if that particular station is not representative of the region then the entire trend can be unrepresentative of the region. Precipitation statistics also contain a large amount of natural variability, as seen in the time series analysis in the next chapter of this thesis. It is also the reason why some of the standard forecast verification statistics are insufficient to describe the properties of precipitation.

Precipitation is notoriously difficult to analyse and model. For this reason it is still the subject of numerous scientific studies every year despite the fact that scientists have been interested in and collecting precipitation data for over 400 years. This thesis will cover the aspects of precipitation that are most important for the prediction of precipitation extremes within the Waikato River catchment, particularly for the purpose of mitigating the effects of those extremes by providing useful precipitation forecasts. 
Dravitzki, 2009 
CHAPTER 3

\section{CLIMATE}

This chapter examines the precipitation climatology of the Waikato River catchment and the statistical variability of precipitation extremes since 1900. This provides context for future quantitative precipitation forecasts and investigates the relationship between precipitation extremes and the global-scale circulation variability.

With the increased interest in climate change the Intergovernmental Panel for Climate Change (IPCC) recommends a number of indices* to characterise the climate and identify variations. Previous studies (Griffiths, 2007; Salinger and Griffiths, 2001; Manton et al., 2001) applied these indices to station 2101 (Ruakura, see Figure 3.1) in the Waikato. No statistically significant precipitation trends were observed at station 2101 , despite small significant trends being calculated for the areas to the east (decreasing precipitation totals) and west (increasing) of this station in previous literature. In this chapter a regional time series was analysed for changes in the rate of occurrence and size of extreme precipitation events between 1900 and 2006 for this apparently neutral region.

To represent the volume of precipitation, individual daily rain gauge observations within this catchment are averaged (see Figure 3.2 for rain gauge station locations). This varies from previous research of precipitation extremes in New Zealand (e.g. Griffiths (2007) or Salinger et al. (2001)) that used single stations to represent regions of the country. However, a single chosen station may not represent all relevant events within the catchment, e.g. widespread precipitation of moderate intensity that can re-

*http://ccama.seos.uvic.ca/ETCCDMI/indes.shtml 
sult in a larger volume of water entering the river compared to intense precipitation only occurring in a localised area. Alternatively, heavy convective precipitation in the region may fail to record any precipitation at the recording site.

Events during the 20th century have also been analysed for fluctuations in the frequency of occurrence and size of the event. Climate variations in New Zealand have been correlated with: the El Niño-Southern Oscillation (ENSO) phenomena e.g. Salinger and Mullan (1999) (and the interrelated Interdecadal Pacific Oscillation (IPO) e.g. Salinger et al. (2001)); the Southern Annular Mode (SAM) e.g. Kidston et al. (2009); and global warming e.g. Manton et al. (2001). This chapter studies correlations between these climate phenomena and extreme precipitation events in the Waikato. A number of studies have examined climate variation in New Zealand (e.g. Griffiths (2007); Griffiths et al. (2003); Manton et al. (2001); Salinger and Griffiths (2001); Salinger and Mullan (1999); Mullan (1995); and Salinger et al. (1995)), often concluding that climatic variations are correlated to large-scale circulation variations (Salinger and Mullan, 1999) associated with a change in the strength of the westerly wind belt.

\subsection{Data}

Daily precipitation and monthly average temperature data for the Waikato catchment (and surrounding areas) were obtained from the New Zealand Climate Database (CLIDB) maintained by the National Institute of Water and Atmosphere (NIWA). The locations of stations explicitly mentioned in this chapter are shown in Figure 3.1. Temperature is included in this climatology because of the suggestion (Trenberth et al., 2003) that precipitation changes are related to temperature changes.

Interdecadal Pacific Oscillation (IPO) monthly indices ${ }^{\dagger}$ were obtained from the Joint Institute for the Study of the Atmosphere and Ocean (JISAO). El Niño-Southern Oscillation (ENSO) monthly indices ${ }^{\ddagger}$ (in the form of the multivariate ENSO index

\footnotetext{
${ }^{\dagger}$ http://www.jisao.washington.edu/pdo

${ }^{\ddagger}$ http://www.cdc.noaa.gov/people/klaus.wolter/MEI/mei.html
} 


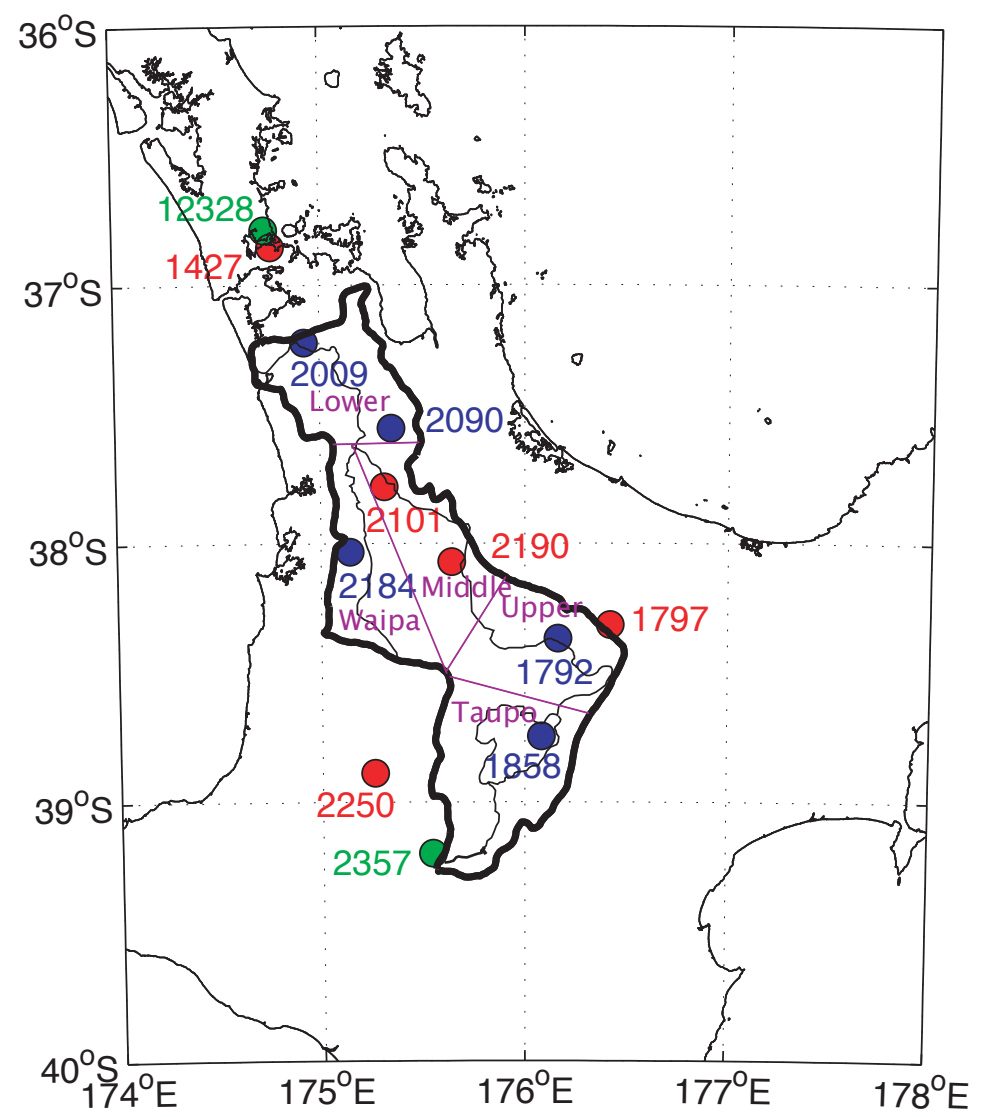

Figure 3.1 The location of rain gauge stations explicitly mentioned in Chapter 3. Blue circles indicate the gauges shown in Figure 3.5 and red circles show those in Figure 3.10. The green circles are the temperature stations mentioned in section 3.2. The agent number of each station is provided and further details about each station are provided in Appendix A. Black lines show the Waikato River catchment boundary and major rivers, and the approximate subcatchment boundaries and names are in purple.

(MEI)) and the Southern Annular mode (or Antarctic Oscillation)§ were downloaded from the Earth System Research Laboratory (ESRL).

\subsection{Climate}

The climate of the Waikato is described as temperate, and is moderated by the close proximity of the ocean (Garnier, 1950). Climate stations are unevenly distributed throughout New Zealand, with many more stations located in low-lying and coastal areas.

At stations within the Waikato River catchment the mean annual precipitation totals range between 1,200 $\mathrm{mm}$ and 2,500 $\mathrm{mm}$ per year (see Figure 3.2), and in any year these values can vary by $\pm 300 \mathrm{~mm}$. A complete list of these stations, including their mean annual precipitation totals, is provided in Appendix A.

\footnotetext{
$\S$ http://www.cdc.ncep.noaa.gov/products/precip/CWlink/ daily_ao_index/aao/aao.shtml
} 


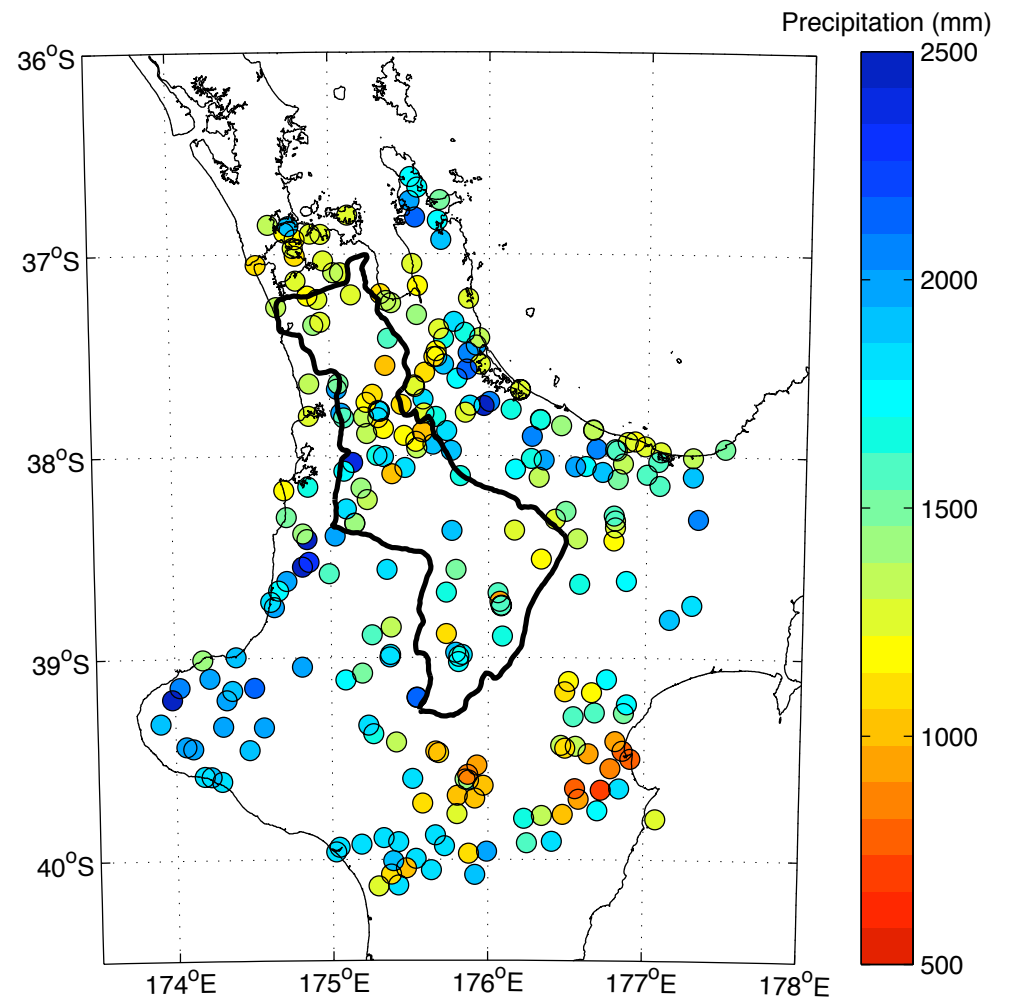

Figure 3.2 Mean total annual precipitation recorded at individual rain gauge stations over the period 1996 to 2007 in the central North Island. See Appendix A for station details.

Diurnal or seasonal fluctuations in temperatures are influenced by the proximity to the ocean. The arithmetic average of the monthly mean temperatures over the 1996 to 2007 period were calculated at stations near the Waikato. Figure 3.3 shows the mean monthly temperature ranges between 10 and $20^{\circ} \mathrm{C}$, with the lowest monthly mean of $1.5^{\circ} \mathrm{C}$ on Mt Ruapehu (station 2357) in August 1992, and the highest monthly mean of $24.2^{\circ} \mathrm{C}$ north of Auckland (station 12328) in February 1998.

\subsection{Regional time series}

Regional time series are calculated for both monthly temperature and daily precipitation data.

\subsubsection{Temperature}

Many established techniques exist for interpolating between temperature stations such as Zheng et al. (1997) or Trewin and Trevitt (1996). However, the regional temperature 


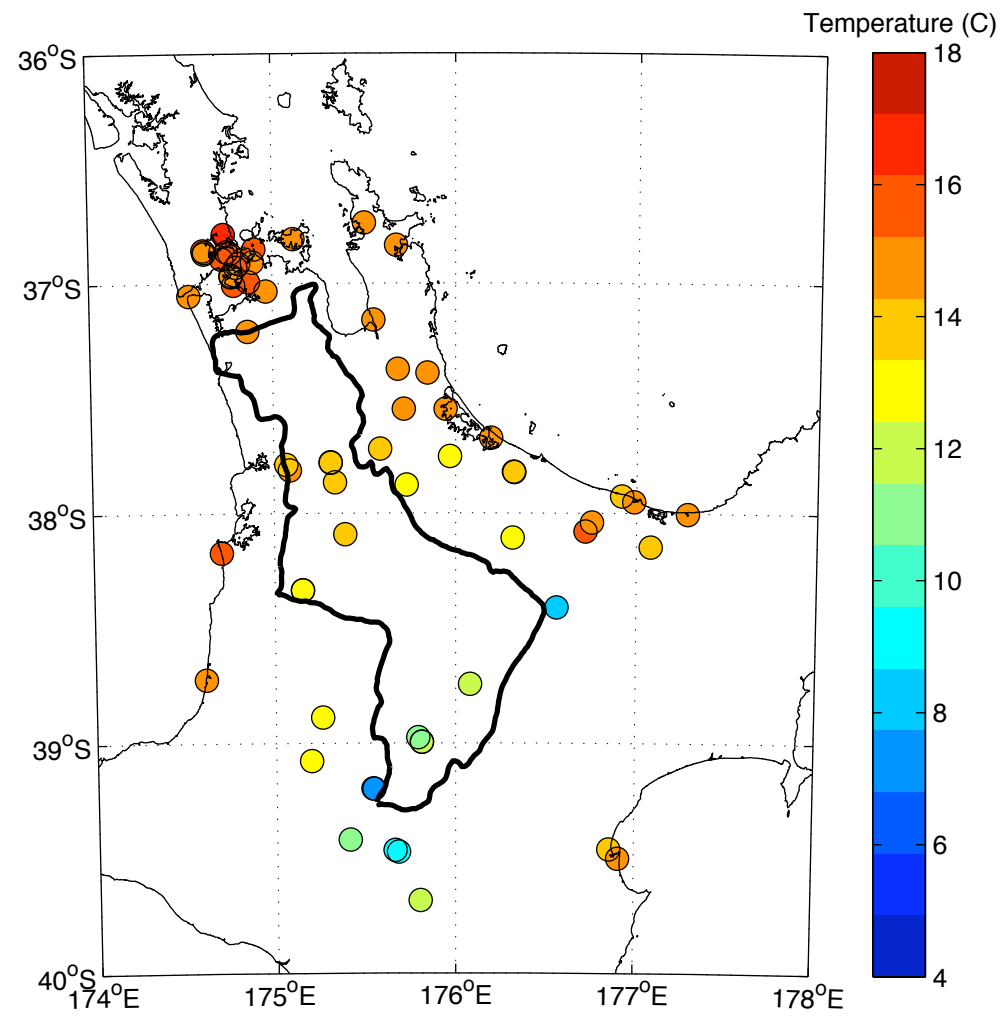

Figure 3.3 Means of the mean monthly temperature recorded at observation stations over the period 1996 to 2007 in the central North Island. See Appendix A for station details.

time series $\left(\overline{T_{m}}\right)$ in this study was calculated through simultaneous linear regressions between each station and the regional mean for each month as in equation 3.1. This process should reduce the effects of missing data. Available monthly temperature averages were extracted for up to 155 stations in and around the Waikato for the period 1900 to $2005 .^{\top}$

The monthly temperature value $\left(T_{m}^{s}\right)$ at each station $\left({ }^{s}\right)$ for each month $\left.{ }_{m}\right)$ was linearly related to the regional monthly temperature value $\left(\overline{T_{m}}\right)$ by the equation:

$$
T_{m}^{s}=A^{s} \overline{T_{m}}+B^{s}
$$

where A is a scaling factor and B is a shift. Systematic variations in temperature can be caused by altitude, latitude and slope variations between stations. The parameters A and B should allow for the combined effect of these systematic variations. To normalise the parameters, the average scaling factor over all stations was set to one, while the

\footnotetext{
IThe varying data time periods in this thesis are due to the availability of data when the analysis took place.
} 
sum of the shifts was set to zero. These coefficients were derived for each station using iterative least squares. Once the monthly time series was estimated, the mean value for the year was calculated to remove seasonal trends, and the linear trend over the entire period was calculated using a normal distribution maximum likelihood estimation (Wilks, 1995, or Appendix B for details of the calculation).

\subsubsection{Precipitation}

The daily precipitation data were organised into two data sets due to poor station coverage in the Waikato catchment during the earlier decades of the 20th century. The limited number of stations (18) with longer records were used to examine long-term trends and changes in precipitation extremes, while a more dense station network (253 stations) was used for the recent climatology and for case study analysis of recent heavy precipitation events in Chapter 4.

In this study, precipitation events are identified by volume, so both spatial and temporal non-weighted arithmetic averaging was used. The approach was inspired by Houze et al. (1990), where an extreme event was defined as at least $25 \mathrm{~mm}$ of precipitation in 24 hours over an area exceeding $12,500 \mathrm{~km}^{2}$, but modified to suit our catchment; similar criteria have also been used in Smith et al. (1994); Bradley and Smith (1994); and Schumacher and Johnson (2006). This is a simplistic form of spatial interpolation and more complicated techniques such as Veronoi polygons were not required in this study as this simple method identified the 'problematic events' (see section 3.3.2 for definition) for this catchment.

The long-term daily precipitation time series included data from all of the 18 stations in or near the Waikato catchment that had at least 50 years of daily data between 1900 and 2007. Even with this 50-year criterion, 50\% of observations over the 107 -year period were unavailable. Prior to 1950, there were no stations at higher altitudes within the region; Figure 3.2 illustrates that higher mean annual precipitation totals were observed at these stations. The inclusion of the Mt Ruapehu station in 1950 sig- 
nificantly skewed the precipitation time series to higher values producing false trends. This station was therefore excluded from the analysis, but this example indicates that the precipitation values in the time series are negatively skewed in relation to the actual precipitation due to the lack of high altitude rain gauges. The effect of sparse data on the regional time series is further discussed in section 7.4.

The recent daily precipitation data included observations from up to 253 stations that recorded data between 1996 and 2007. On average, over the 253 stations only $40 \%$ of days contained precipitation observations. Thus the time series used here is calculated from the 21 stations within the catchment boundary that were at least 85\% complete. See Figure 3.4 for the locations of stations that met the inclusion requirement.

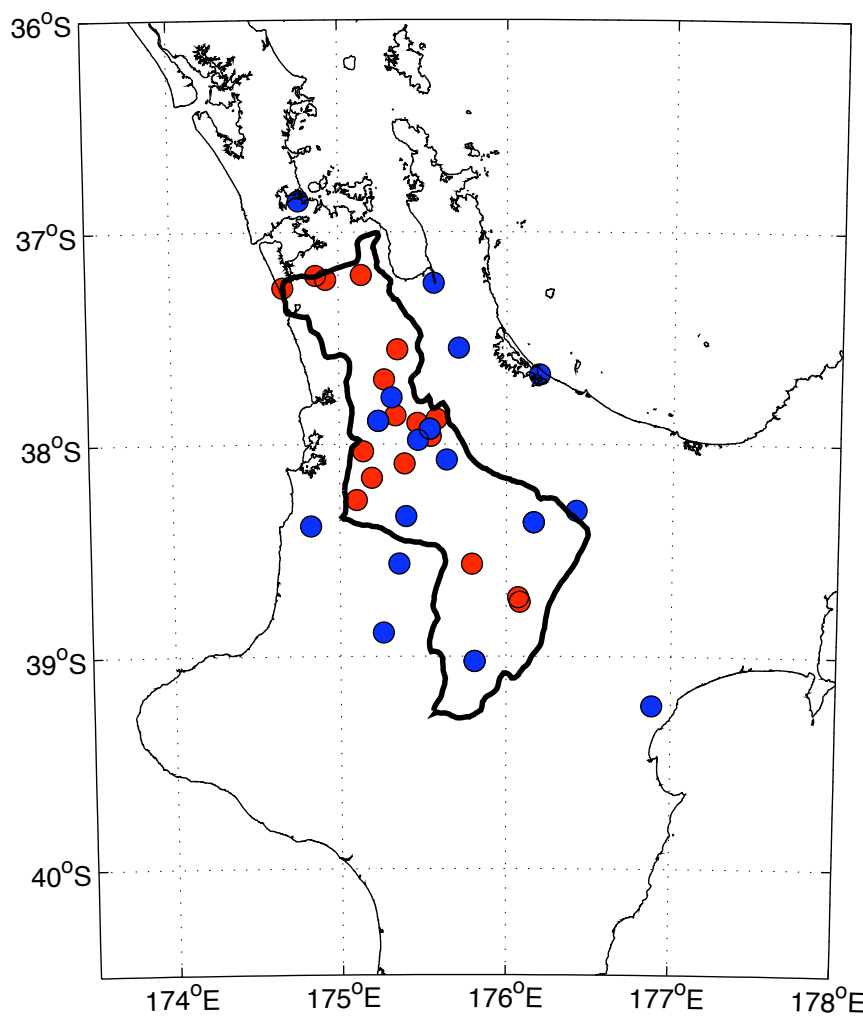

Figure 3.4 The locations of stations that met the completeness criteria to be included in the regional time series. Red circles indicate those stations included in the 'recent' time series and blue circles are stations included in the 'long-term' time series.

\section{Missing data}

To prevent a skew caused by missing data, missing observations at stations that met the completeness criteria above were in-filled. Each missing datum was substituted by 
the closest precipitation observation for that period (also done in Falvey and Garreaud (2007)). The method of choosing the closest observation is based on a k-means cluster analysis distance equation (Marzban and Sandgathe, 2006; Wilks, 1995) over four variables longitude, latitude, altitude, and mean precipitation. Specifically:

$$
\text { distance }=\sqrt{\left(\left(x_{1}-x_{2}\right) * \alpha_{l o n}\right)^{2}+\left(\left(y_{1}-y_{2}\right) * \alpha_{l a t}\right)^{2}+\ln \left(\left(z_{1}-z_{2}\right)^{2}\right)+\ln \left(\left(\overline{p_{1}}-\overline{p_{2}}\right)^{2}\right)}
$$

where $x, y$, and $z$ are longitude (degrees), latitude (degrees), and altitude (m) respectively and $\bar{p}$ is the mean daily precipitation $(\mathrm{mm})$. Latitude and longitude values were converted to distances in kilometres using the approximation that 1-degree latitude is $111 \mathrm{~km}\left(\alpha_{\text {lat }}\right)$ and that 1 degree of longitude is $80 \mathrm{~km}\left(\alpha_{\text {lon }}\right.$; at $\left.38 \mathrm{~S}\right)$. As variations in mean precipitation and altitude are of a larger magnitude than the longitude and latitude, and small variations are less important, natural logs were used to scale both these values in the function. Additional scaling parameters were unnecessary as the weightings seldom altered the station selected as the closest.

In general the interpolation of precipitation between stations is inappropriate (see section 2.2); however, as noted above, if a station with a significantly different mean is only included in part of the time series (e.g. the Mt Ruapehu station), then the regional time series may be skewed. To test the appropriateness of this substitution, periods of overlapping data between a selected station and a replacement station were compared. A root mean square error (RMSE) calculation between overlapping observations of replacement stations showed $67 \%$ of precipitation observations were within $2 \mathrm{~mm}$ of each other. Although this adds a small uncertainty to the regional time series, the associated errors should be less than if the missing data were ignored. 


\section{The regional time series}

As discussed in section 2.2, the precipitation recorded at a single station is not necessarily representative of the volume of water precipitated within the entire catchment region (with an area of $\sim 14,000 \mathrm{~km}^{2}$ ). This is demonstrated if we consider the individual time series for the year 2002 (Figure 3.5) at the stations identified in Figure 3.1. The dates where the station precipitation value exceeded its 95th percentile vary

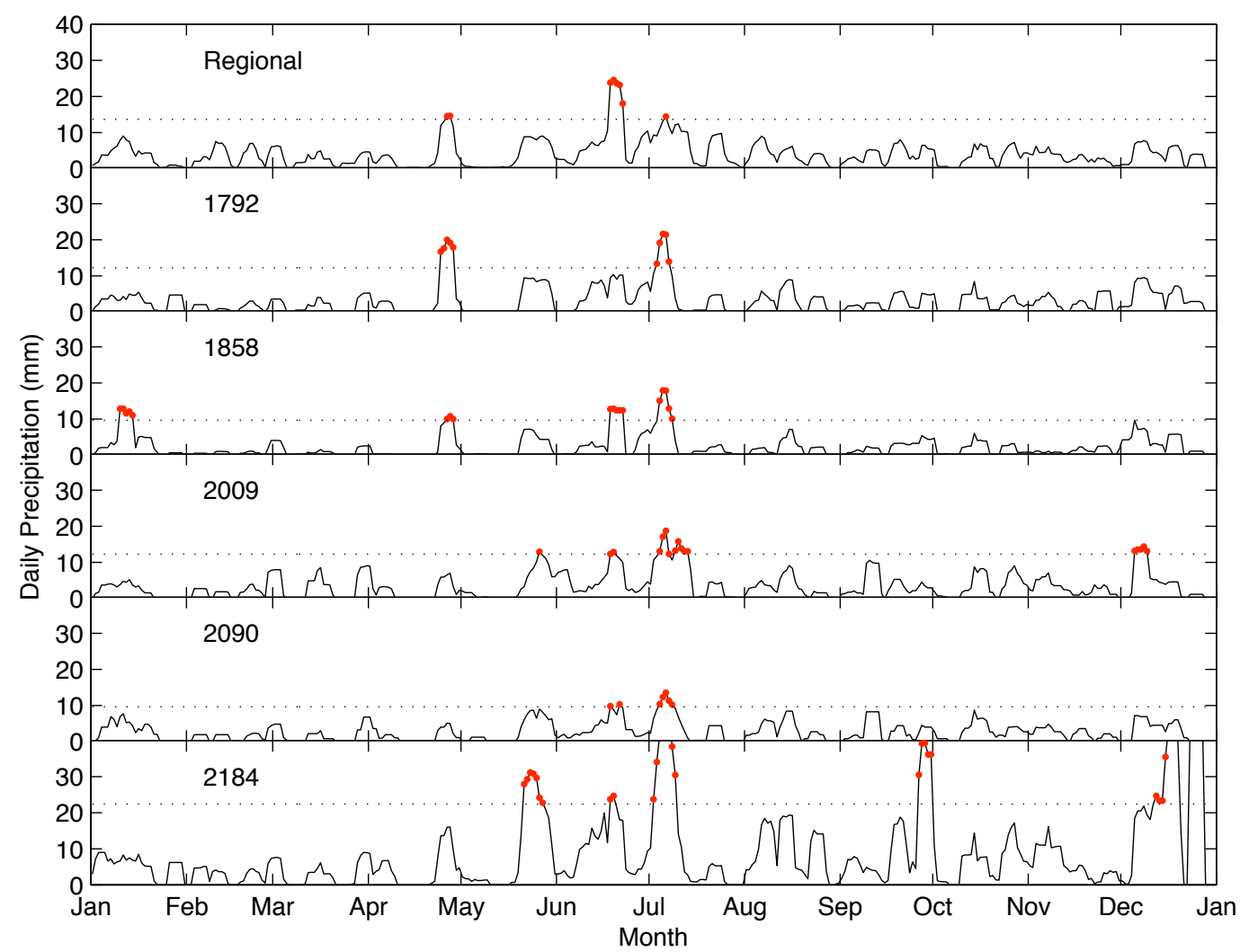

Figure 3.5 The variation in daily precipitation totals at different stations within the Waikato River catchment for the year 2002. Dots along the time series represent values exceeding the 95 th percentile in this and following figures. The regional integration, in the top panel, shows that peaks recorded at multiple individual stations are present in the regional time series. The station identification numbers are shown in each panel and stations are located in Figure3.1 and in Appendix A.

between stations. The regionally averaged time series (top panel of Figure 3.5) has identified the common peaks between stations such as 20 June and 6 July. However the isolated peak on 28 September at station 2184 does not exceed the 95th percentile at other stations nor in the regional time series. 
Floods arise due to the combined effect of precipitation and ground saturation (Roper, 2001). Therefore identifying extremes from multi-day accumulations could improve the identification of such events. A running average smoothing function was applied to the regionally integrated daily time series (see Figure 3.6). A five-day

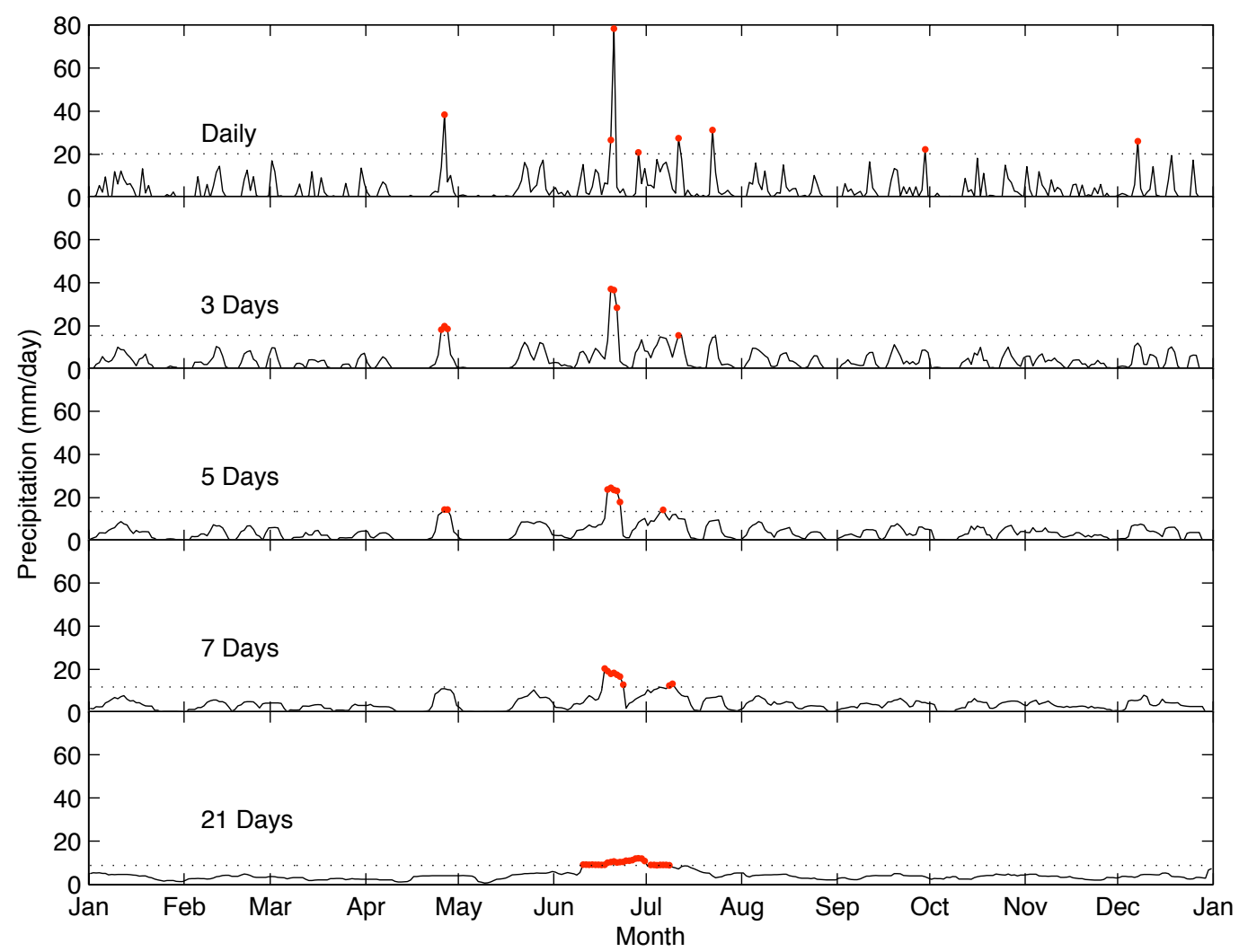

Figure 3.6 The effects of varying the smoothing period for the area averaged time series for 2002. Days exceeding the 95th percentile in each time series are identified by dots. The panels from top to bottom represent daily, 3 day, 5 day, 7 day and 21 day smoothing parameters.

smoothing was chosen because periods of less than five days only selected the highest intensity peaks, missing the persistent events that occurred over multiple days. Longer smoothing periods did not identify the extremely intense events, known to have been 'problematic' (see section 3.3.2), such as the April event identified in the five-day time series but not the seven-day time series. In this example period, similar events were identified in the three-day and five-day time series. However, soil can remain saturated for longer than five days, meaning that additional water in this period is more likely to contribute to surface run-off. Given the size of the catchment, surface run-off will take 
less than two days to enter the river, and water in Lake Taupo takes approximately 40hours to reach the sea if unimpeded (Roper, 2001).

Thus the regional precipitation time series in this study are spatially averaged, fiveday smoothed time series (Figure 3.7 shows the complete regional time series over the recent time period).
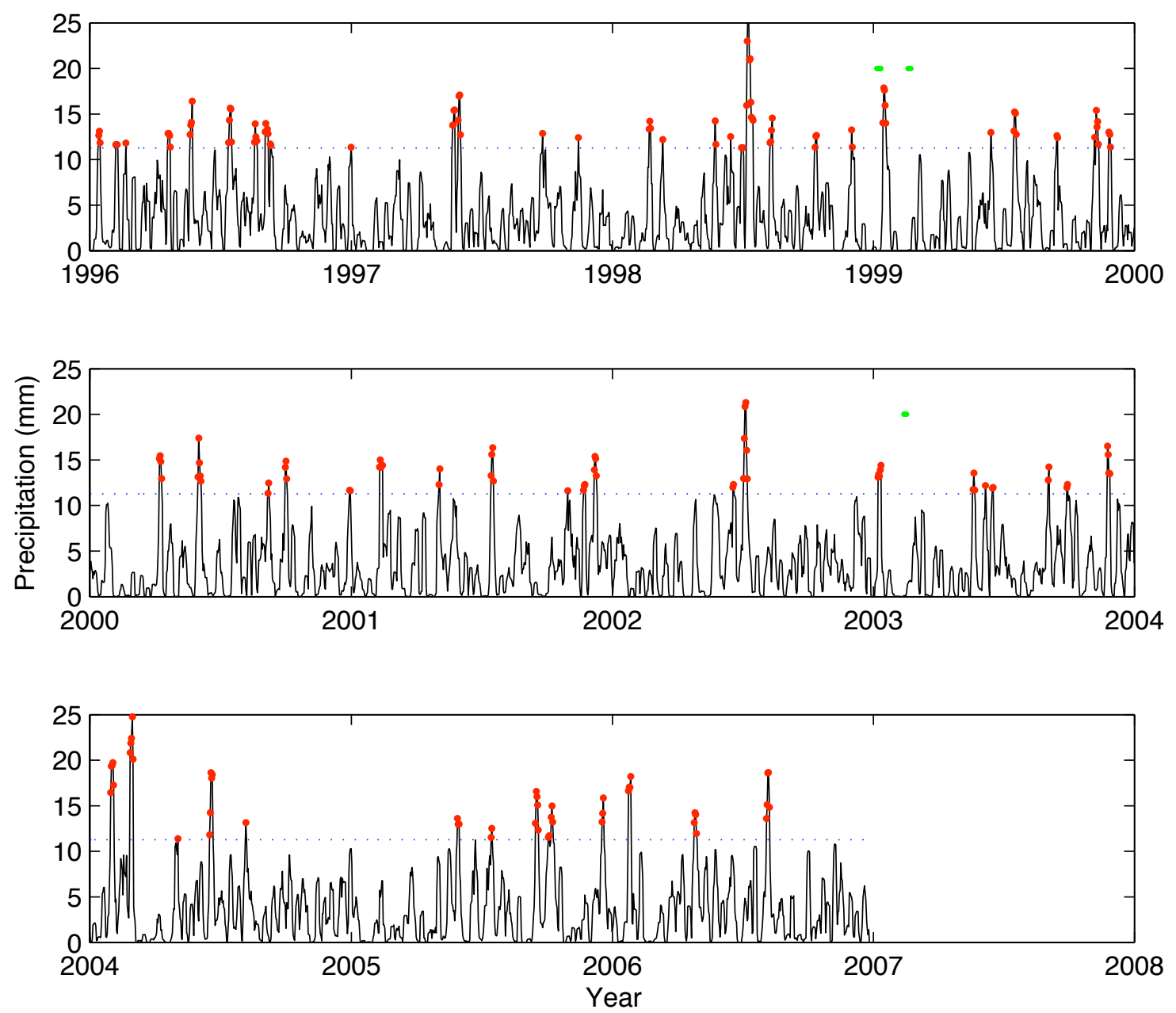

Figure 3.7 The complete regional time series calculated from the spatially dense 'recent' data set. This is an area-averaged, five-day smoothed time series plotted for each day. Precipitation extremes are marked by dots, red indicating precipitation exceeding the 95th percentile, and green showing periods identified as droughts. 


\section{Heavy precipitation events}

Heavy precipitation is defined statistically using percentile threshold values. This is consistent with the IPCC climate indices of precipitation extremes. Ninety-fifth and 99th percentiles were also used as threshold values in previous New Zealand studies by Griffiths (2007); Griffiths et al. (2003); Manton et al. (2001); and Salinger and Mullan (1999). Figure 3.8 shows the precipitation value threshold and the number of events identified as a function of percentile. Where consecutive days exceed the threshold,
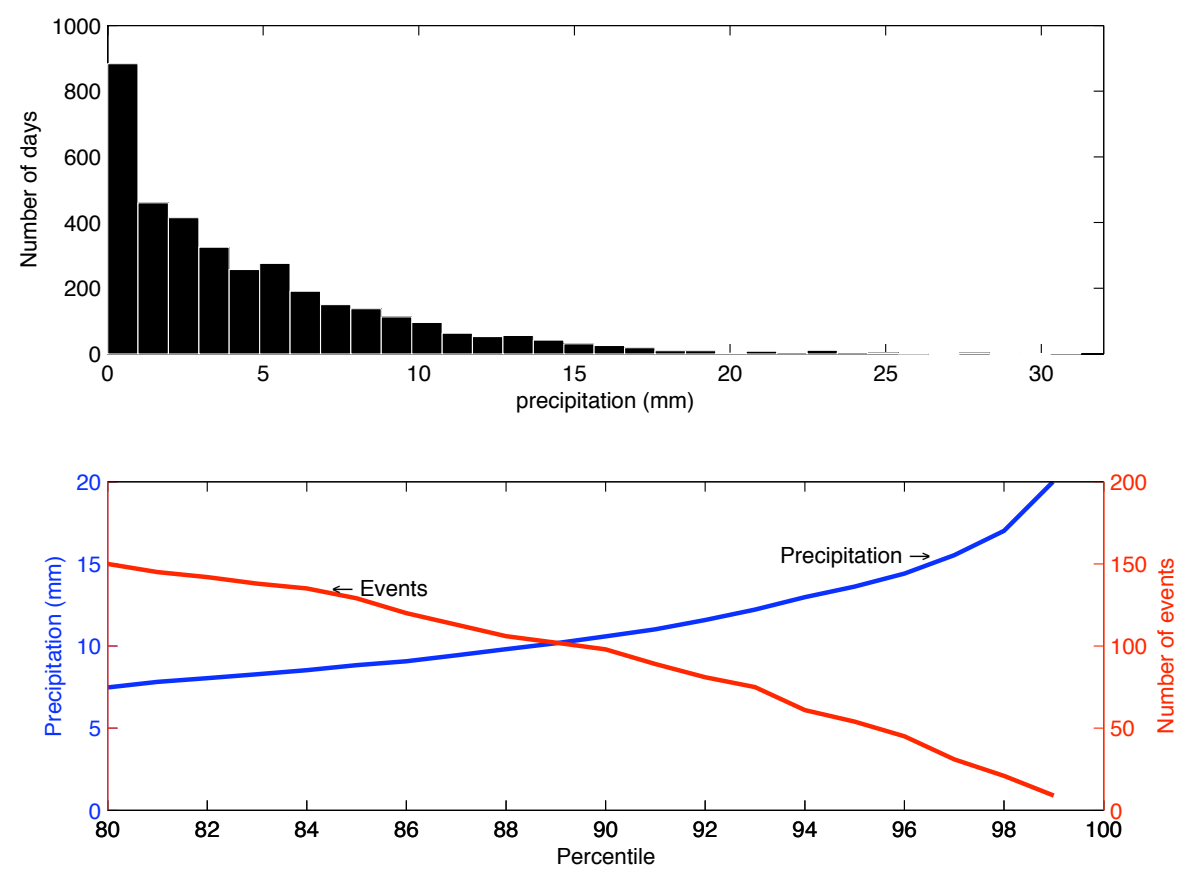

Figure 3.8 Top panel: A histogram of daily precipitation values, over the recent time period 1996-2007, in each $\mathrm{mm}$ range bin. Lower panel: the precipitation threshold value and the number of events this corresponds to for each percentile threshold value between 80 and 99 . Consecutive days over the threshold are grouped together to form a single event.

these are considered to be a single event. In the future quantitative precipitation forecast values could also be compared to these percentile thresholds to determine the comparative severity of a coming event.

In this study we define the frequency of occurrence as the number of events identified per year; the duration is the number of consecutive days exceeding the threshold for the event; the volume is the cumulative regional time series value over the days identified in 
the event; and the mean intensity of an event is the total precipitation volume divided by the duration. Intensities and volumes are expressed in millimetres and millimetres per event of precipitation throughout this paper, applying the common notation in precipitation literature of shortening millimetres per unit area to millimetres.

\section{Drought events}

In this study droughts are defined if the sum of precipitation over the previous 15days of the regional time series is less than $1 \mathrm{~mm}$. This is consistent with the British climatological definition of an absolute drought; "fifteen days during which no measurable daily precipitation has fallen" . The drought continues until the conditions are no longer satisfied.

This drought definition is also consistent with the 'effective storage capacity' of Lake Taupo (Roper, 2001). This means that if the operations were run at a normal level for two weeks without precipitation the storage water in Lake Taupo would have fallen from a mean level to the minimum operating level (this is the definition of effective storage capacity here but can also be the difference between the maximum and minimum levels). Generally conservation efforts would be applied before the lake reaches the minimum level, but 15 or more days of continuous dry conditions will usually constrain the hydroelectric operation.

\section{Observed events}

The heavy precipitation events and drought periods objectively identified through this process were compared to subjective reports of problematic weather. Problematic weather, in this study, is any media report indicating that there was damage or inconvenience caused by the weather. The subjective list of problematic weather reports was compiled through local and national newspaper archives, and lists published by the Meteorological Service of New Zealand Ltd (MetService) and the New Zealand

\footnotetext{
"Glossary of meteorology, http://amsglossary.allenpress.com/glossary/
} 
Meteorological Society. Each report in the list corresponded to an event identified as above the 95th percentile of the regional time series. Additionally, every event above the 98th percentile of the regional time series corresponded to a problematic weather report. This indicates that the objective method applied here identified all the periods where precipitation has caused problems, without identifying excessive events that did not cause problems. The additional events in the objective list may have been problematic if they had occurred when ground conditions had been more saturated, or in areas of denser population.

It is assumed that including more stations in the calculation of the regional time series would lead to it being more representative of the regional precipitation volume. However, there are only eight stations within the catchment area with a long-term observation record. Because of this, it was decided to include stations outside the catchment in the 'long-term' time series (Figure 3.4). The additional stations increased the intensity of extreme precipitation events because this included stations that observe larger extremes than are observed within the Waikato catchment.

To examine the consistency in the events identified, the overlapping time period of long-term time series was compared to the 'recent' time series. As the previous studies (Griffiths, 2007; Salinger and Griffiths, 2001) of precipitation extremes in the Waikato used station 2101 Ruakura to determine trends, the dates of extreme at this station were also compared to the dates selected from the regional time series used here. The dates that each time series exceeded its 95th percentile during the period 1996 to 2007 were compared. Further investigation of dates that were not identified in both regional time series showed that these dates were borderline events in both time series. When comparing the regional time series to station 2101 only $35 \%$ of the same dates were identified, and there were numerous events that, while large at 2101, were well below the regional time series threshold, and vice versa. This could be due to the extent of the precipitation; either localised intense precipitation at 2101, or 2101 being outside the area affected by heavy precipitation. Either way, this station is insufficient by itself to approximate the volume of precipitation in the Waikato River catchment. 
The long-term time series was also compared to available flooding records. Roper (2001) listed the month and year of all 18 significant floods between 1950 and 2000. The 95th percentile threshold did not identify two floods: September 1996 and October 1988. Further these events contained approximately two weeks of continuous moderate precipitation just below the 95th percentile threshold value and would still not have been identified as events if a different smoothing length had been applied to the time series. In terms of river management, the gradual saturation and flooding from the moderate precipitation allowed time for the river management to respond as the event occurred. Operators know that this level of complete saturation will mean that any precipitation will run off into the river.

\subsection{Long-term variations}

Data between 1900 and 2006 were examined for any longer-term trends and to ascertain any possible variations in the Waikato climate. Annual precipitation totals, annual temperature means, extreme precipitation event characteristics, and drought event characteristics have all been examined for the presence of trends. New Zealand is too small to be adequately represented in global studies of precipitation variation such as Alexander et al. (2006) or Dai et al. (1997), but it has been suggested that globally the nature of precipitation may change with increased temperature due to global warming (Trenberth et al., 2003).

\subsubsection{Annual precipitation totals}

Trends in precipitation are frequently summarised through analysis of annual totals (e.g. Salinger and Mullan (1999, in New Zealand); or Türkes (1996, in Turkey)) but significant trends are uncommon. In this study the annual precipitation total was calculated from the long-term regional time series (Figure 3.9a). Similar plots for five stations identified in Figure 3.1 are shown in Figure 3.10. The average number of stations recording data during each year is shown in Figure 3.9c. The lack of observations 

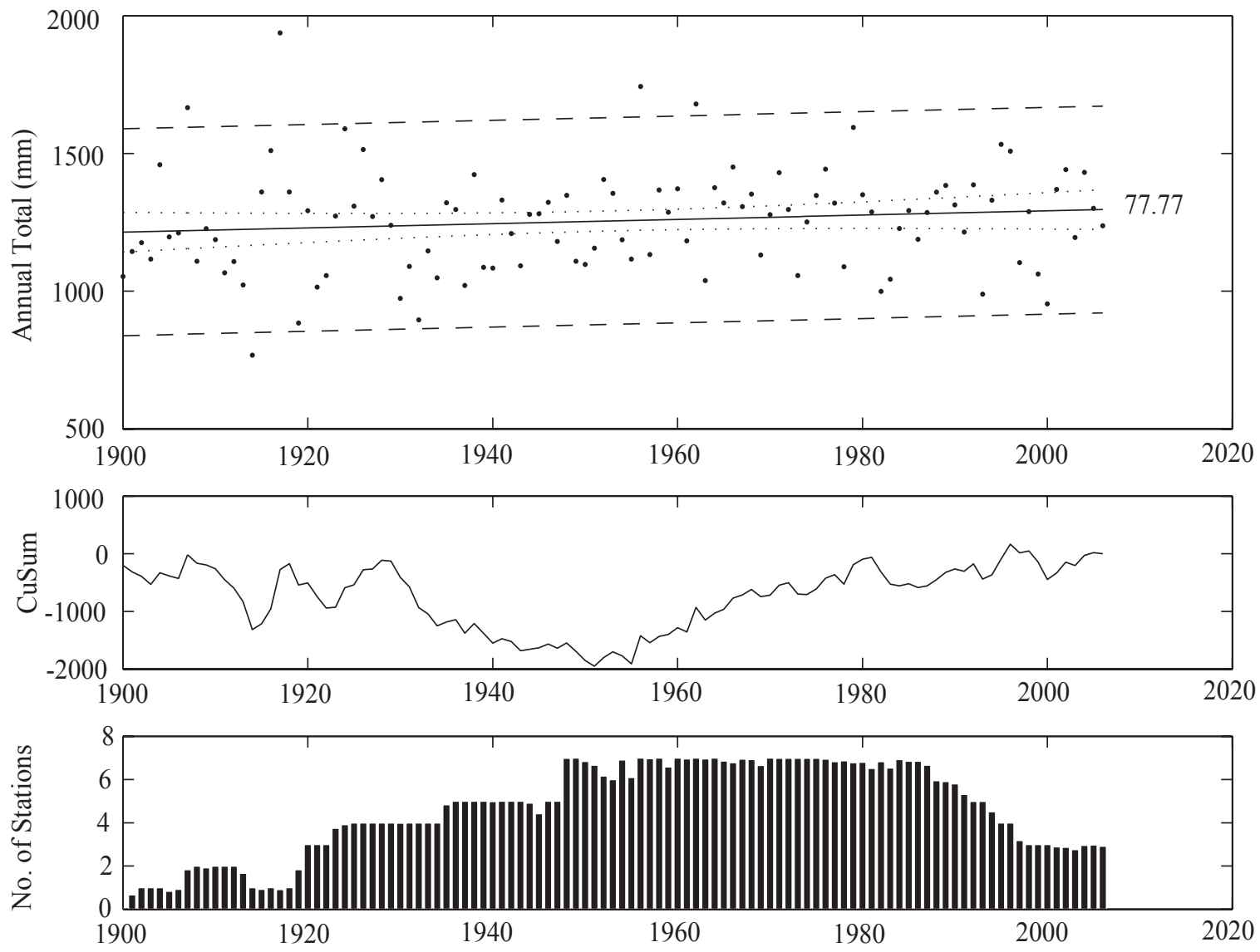

Figure 3.9 These three panels show (a) annual total precipitation calculated from the longterm regional time series; (b) the cumulative summation of the above time series; and (c) the average number of stations contributing observational data for each year. The solid line in the top panels shows the best-fit linear trend line (corresponding to an insignificant increase in annual precipitation of $77 \mathrm{~mm}$ per year), with the $95 \%$ confidence range dotted. The dashed line represents two standard deviations from the trend line.

at both ends of the time series are potential sources of error.

The cumulative summations (CuSum) indicate where a linear trend changes by a change in the gradient of the CuSum. The CuSum is defined by equation 3.3:

$$
S_{i}=S_{i-1}+\left(X_{i}-\bar{X}\right)
$$

where $\mathrm{S}_{i}$ is the CuSum at a point in the time series and $\mathrm{X}$ is the time series over which it is being calculated. Plots 3.9(b) and the right hand plots of Figure 3.10 all show that the annual precipitation totals have changed at an inconsistent rate over the period. 


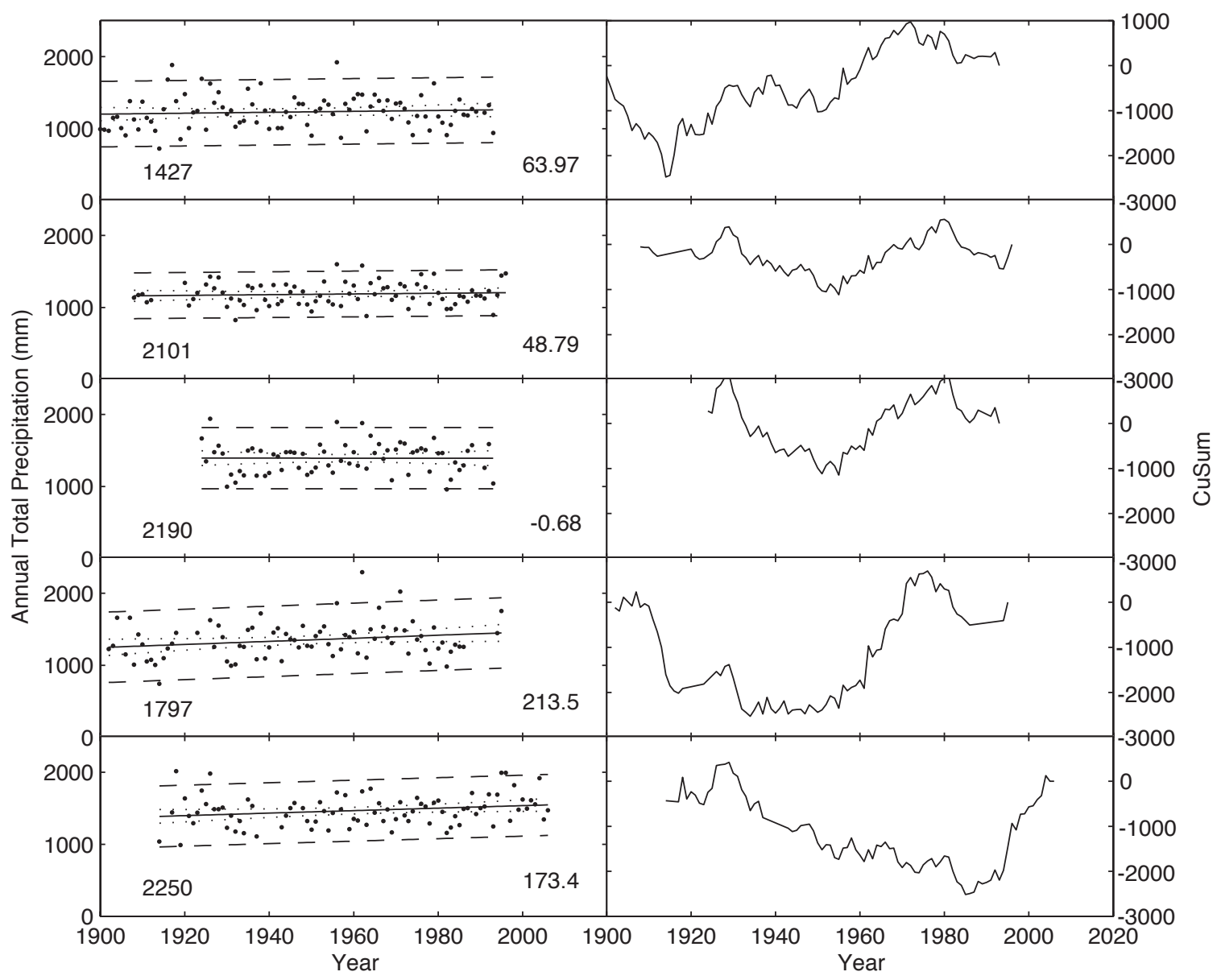

Figure 3.10 The total annual precipitation trends for the selected rain gauges identified in Figure 3.1. To the right of each trend is the cumulative summation for the time series. Bestfit linear trends were calculated for each time series and are plotted in a solid line. The value in the lower right corner of each time series represents the increase in annual precipitation over a century according to the trend line.

Four of the individual stations show a change in rate around 1950 that is also seen in the regional cumulative summation and coincides with a shift in general circulation identified by Salinger and Mullan (1999).

Annual totals have increased by a statistically insignificant (at a 0.05 level) $77 \mathrm{~mm}$ over the period. The individual stations around the region have increases ranging from $-0.6 \mathrm{~mm}$ to $213.5 \mathrm{~mm}$ over the period. There is considerable variation about the trend line in each time series plot. Only station 2250 (Taumarunui, technically outside the catchment) has a significant positive trend at the 0.05 confidence level (consistent with the value obtained by Griffiths (2007) for the same station) showing a general increase 
of $173 \mathrm{~mm}$ over 100 years. The lack of widespread significant trends indicates that there is currently no evidence that there has been a linear increase in annual precipitation totals.

\subsubsection{Annual temperature means}

Changes in precipitation extremes have been related to mean temperature increases (e.g. Alexander et al. (2006), Trenberth et al. (2003), and Trenberth (1999)). Therefore a mean annual temperature time series for this region has been calculated from all 155 temperature stations. The complete regional temperature time series is shown in Figure 3.11 .

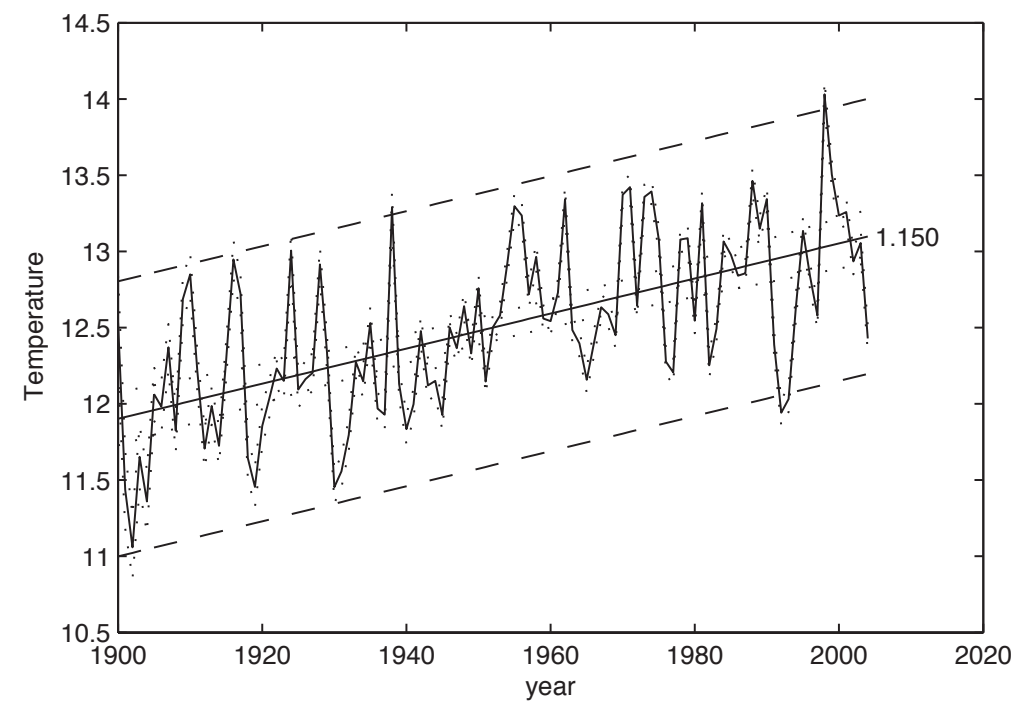

Figure 3.11 Mean annual temperature time series for the Waikato catchment. The linear trend (marked by the solid line) shows an increase of $1.15^{\circ} \mathrm{C}$ with the 0.05 confidence level marked by the dotted lines, the dashed lines mark two data standard deviations.

The linear trend line applied to the temperature time series showed a $1.15^{\circ} \mathrm{C} \pm 0.45^{\circ} \mathrm{C}$ increase in temperature between 1900 and 2005 with a 0.05 confidence level. This increase is consistent with other studies calculating temperature increases for NewZ̃ealand (Zheng et al., 1997; Salinger et al., 1995). Even though previous studies used different equations, the results are consistent because the temperature distribution within the region is coherent, and therefore the exact equation used has little impact (Jones and Hulme, 1996). However, no previous studies have found statistically significant temperature trends within the Waikato River catchment. 


\subsubsection{Long-term variations in extreme precipitation}

Trenberth (1999) proposed that globally, extreme precipitation events would account for a larger proportion of annual precipitation as the globe warmed. This is also supported by the 4th IPCC report (IPCC, 2007) expects the change in the intensity of precipitation events to be proportional to changes in total precipitation. This study investigates changes, since 1900, in the rate of event occurrence, duration, intensity and volume (using the definitions provided in section 3.3.2 and Appendix B).

Figure 3.12 shows the number of extreme events in each calendar year. It shows

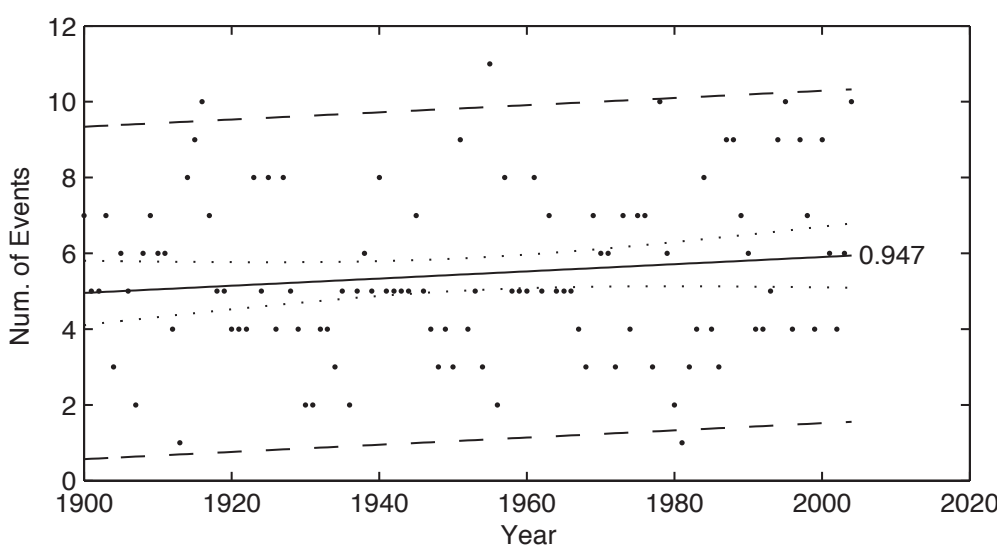

Figure $\mathbf{3 . 1 2}$ The total number of extreme events identified each year since 1900. The solid line shows the best fit linear trend to this data indicating an insignificant $0.95 \%$ increase in the rate of occurrence, while the dotted line shows the 95\% confidence in this trend line and the dashes represents two standard deviations from the trend line.

a statistically insignificant increase of 1.53 events per year over the 100-year period. Salinger et al. (2001) also calculated an insignificant increase in the frequency of events (the magnitude of the increase was not stated) at station 2101, Ruakura.

Box and whisker plots are used to show the median and range of total volume and intensity values for events occurring during each decade. These are then compared to show changes that occurred over the century (Figure 3.13b and d). The sizes of events were also compared to their event duration to examine the relationship between size and duration (Figure 3.13).

When events were subdivided into decades there are few difference between the mean and interquartile ranges of either the total volume or average intensity of events over the century. Further, comparing the outliers in the intensity and volume plots 

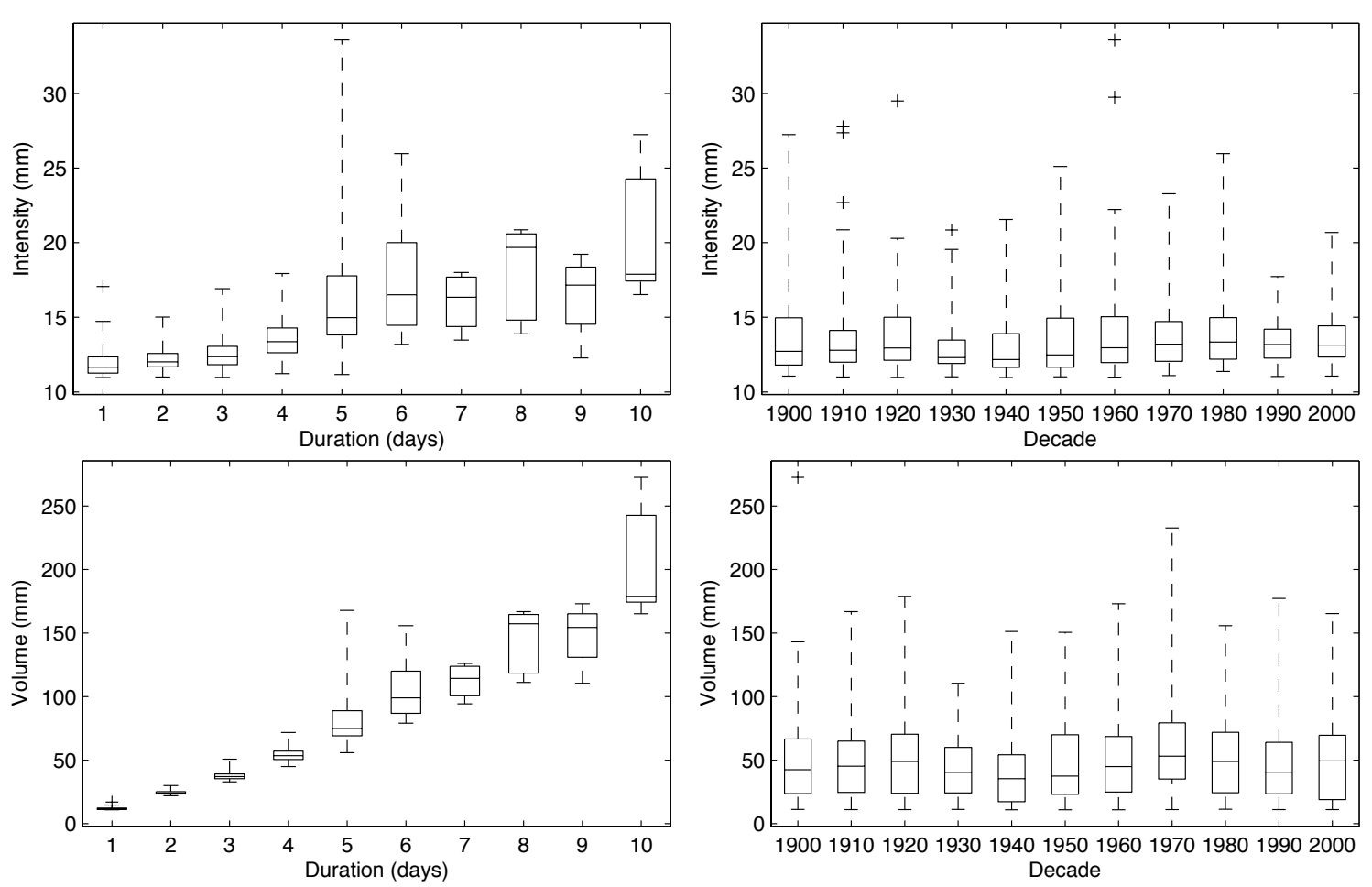

Figure 3.13 These box and whisker plots show the mean, interquartile range and 95th percentiles of intensity ( $a$ and $b$ ) and total volume ( $c$ and $d$ ) of events of different duration (a and $c$ ) and occurring in different decades ( $b$ and $d$ ). Note that the 2000-decade is incomplete.

shows that the highest intensity events (above $25 \mathrm{~mm}$ per day) do not correlate to extreme outliers in the volume. There is no increase in the mean intensity of extreme events seen in our results.

Due to the definition of volume used in this study (see Appendix B), the minimum volume of an event must increase with duration. Events lasting longer than four days show more spread in the volumes recorded. These events also exhibit a wider spread in the mean intensity of an event. The longer duration events also exhibit higher than average mean intensities indicating that these events are not only the biggest in total volume but also in intensity.

\subsubsection{Long-term variations in drought events}

Mullan et al. (2005, using potential evapotranspiration deficit to define droughts) identified an increase in droughts in parts of New Zealand. However, Salinger et al. (2001) 
identified a decrease in the length of dry spells at Ruakura. Figure 3.14 shows the number of droughts that occurred per decade. A simple trend line indicates an insignificant

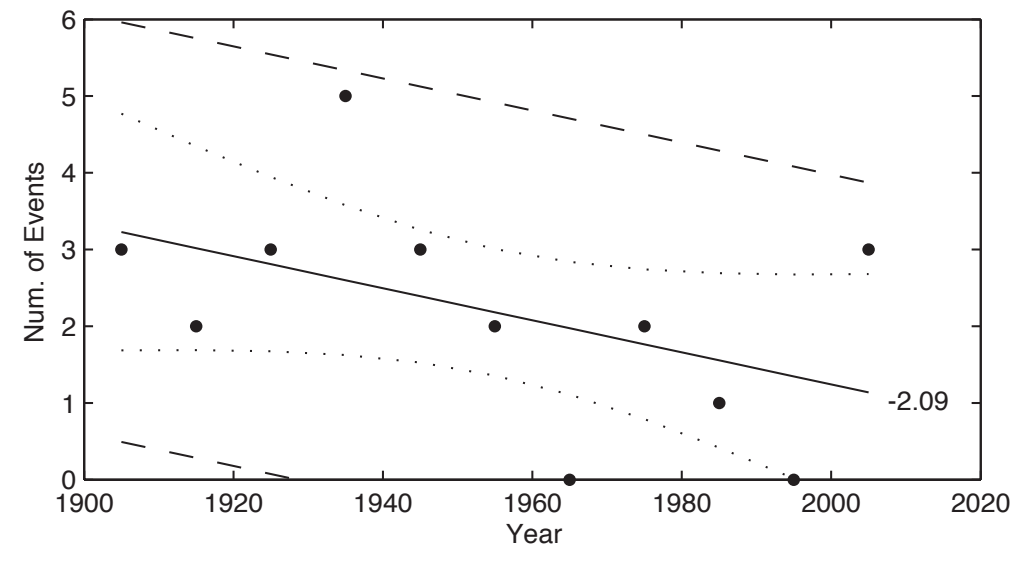

Figure $\mathbf{3 . 1 4}$ The total number of drought events occurring each decade. The solid line shows the bestfit linear trend indicating a $2 \%$ decrease in the rate of occurrence, with the dotted lines showing the 95\% confidence and dashed lines are two standard deviations away.

decrease in the frequency of droughts. However, the low occurrence of droughts means that the statistic is not robust.

As for extreme events, the spread in the duration of the events in each decade are plotted in Figure 3.15. It can be seen that there is no overall trend in drought duration over the time period but there is considerable variation. Again there is no evidence for

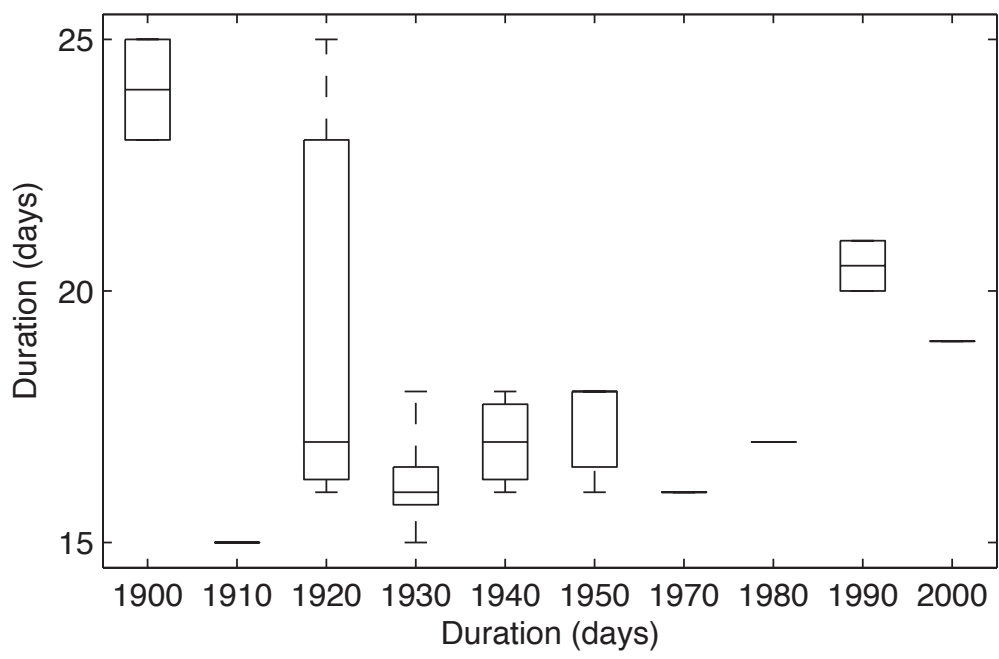

Figure 3.15 The mean and interquartile range of the duration of droughts occurring in each decade.

changes to the duration or frequency of drought conditions over the entire catchment during the last century. 


\subsection{Climate oscillations}

The large-scale atmospheric circulation influences the climate in New Zealand. Variations in some climate variables in New Zealand have been correlated to the IPO, ENSO, and SAM oscillations (Griffiths, 2007; Renwick and Thompson, 2006; Salinger and Mullan, 1999; Salinger et al., 1995; Mullan, 1995), and these oscillations are interconnected (Trenberth et al., 2003; Newman et al., 2003; Salinger and Mullan, 1999). In this study the rate of occurrence and size of extreme precipitation events has been examined for any correlation to these oscillations, although such variations were not obvious from earlier time series plots.

According to Salinger and Mullan (1999, 1995), Griffiths (2007, 2003) and others, negative phases of ENSO result in more frequent and stronger westerly and southwesterly winds over New Zealand which lead to increased precipitation in the west and reduced precipitation in the east, whereas positive phases of ENSO result in increased northerly and easterly winds and decreased precipitation in the west. Negative phases of the IPO are correlated to more frequent negative ENSO phases and vice-versa. A negative SAM index indicates stronger than normal westerly winds and less settled weather in New Zealand, while a positive index corresponds to weaker winds and settled weather (Renwick and Thompson, 2006).

The IPO is defined by the leading principal component of the North Pacific monthly sea surface temperature variability (Mantua et al., 1997) and is available from 1900. ENSO is classified by the Multivariant ENSO Index (MEI) as defined by Wolter and Timlin (1998) and is available from 1950. SAM is calculated from an empirical orthogonal function applied to the monthly mean $1000 \mathrm{hPa}$ height field (Gong and Wang, 1999), and is only available since 1979 when satellite coverage could provide the required highlatitude data.

The MEI combines the elements used to calculate other ENSO indices; such as sea level pressure (used to define the SOI, and used in Salinger and Mullan (1999), Mullan (1995), or Salinger et al. (1995)) or the sea surface temperatures (as in the 
definition of Trenberth (1997)). The MEI is calculated from six climatological variables in the equatorial Pacific; sea level pressure, zonal and meridional components of surface winds, sea surface temperature, surface air temperature, and cloudiness. The inclusion of more atmospheric variables should allow for an index that is more representative of the entire atmosphere and allows calculation of a higher temporal resolution index. Each monthly value incorporates data from the two months leading up to the assigned month, as opposed to the SOI that uses a five-month running mean.

Figure 3.16 shows a tendency for extreme events to cluster around the neutral values of the indices, which is where the largest volume events also occurred. The apparent banding at the lower volume levels of extreme events in all the oscillations are an artefact of the definition of volume applied in this study.

The indices of the three oscillations are independently calculated but are related. The index values of different oscillations are compared during each event in Figure 3.17. The rate of occurrence of events is weakly correlated between the IPO and MEI indices. No other correlations between the oscillation indices for the occurrence of either an extreme event or droughts were found.

There is no evidence of any correlation between extreme events and any oscillation index, limiting the use of these indices as possible seasonal predictors of extreme events in the Waikato River catchment. However, Salinger and Mullan (1999) and Andreoli and Kayano (2005) have calculated significant correlations (linear) between ENSO/IPO and annual/monthly precipitation totals; implying that the variation in total precipitation correlated to these oscillations is caused by a variation in the frequency of light to moderate precipitation.

The frequency of occurrence of extreme events appears to be independent of the phase of these oscillations. To test this independence, theoretical probabilities assuming independence were calculated for each index value of each oscillation and each level of precipitation. The joint probabilities between phase and precipitation amounts for each oscillation are compared to the distribution of observed precipitation extremes 
Severe Events
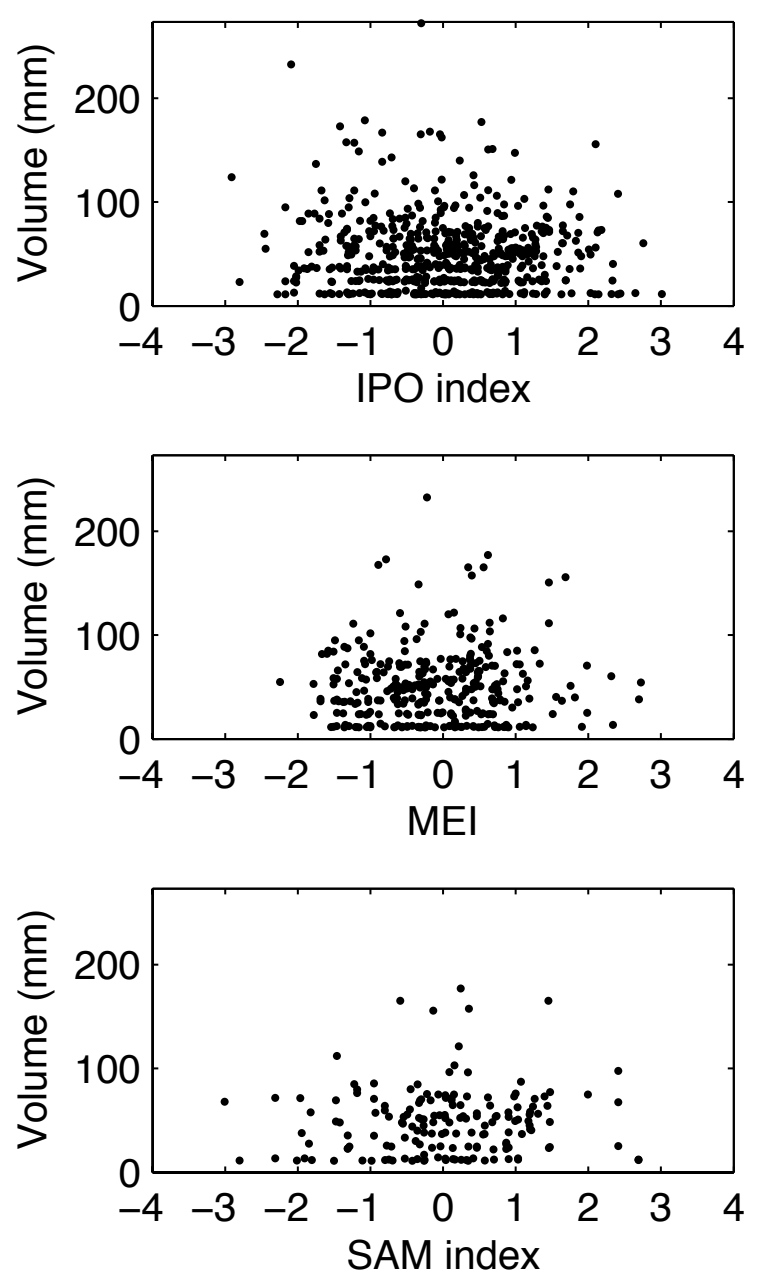

Drought Events
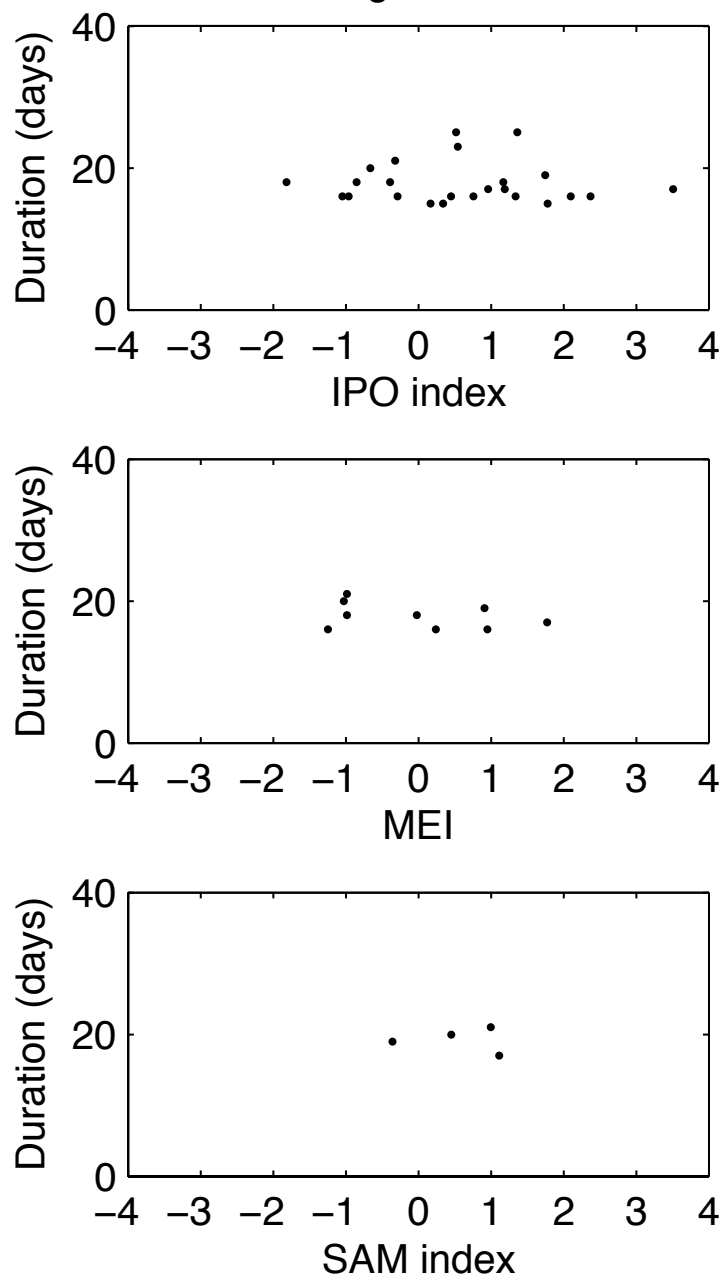

Figure 3.16 The total volume of precipitation during an extreme event and the duration of drought plotted against the index value of large-scale circulations: IPO (since 1900); ENSO (1950); and SAM (1979). The left column of subplots shows the volume of extreme events and the right is the duration of drought events. The rows from top to bottom are: the IPO, the MEI, and the SAM index.

in each phase (Figure 3.18). The similarity between the observed distribution and the joint probabilities indicated that the extremes are independent of these large-scale circulation oscillations.

\subsection{Discussion}

This chapter provided a precipitation climatology for the Waikato River catchment. The long-term time series was used to define the characteristics of events over the 

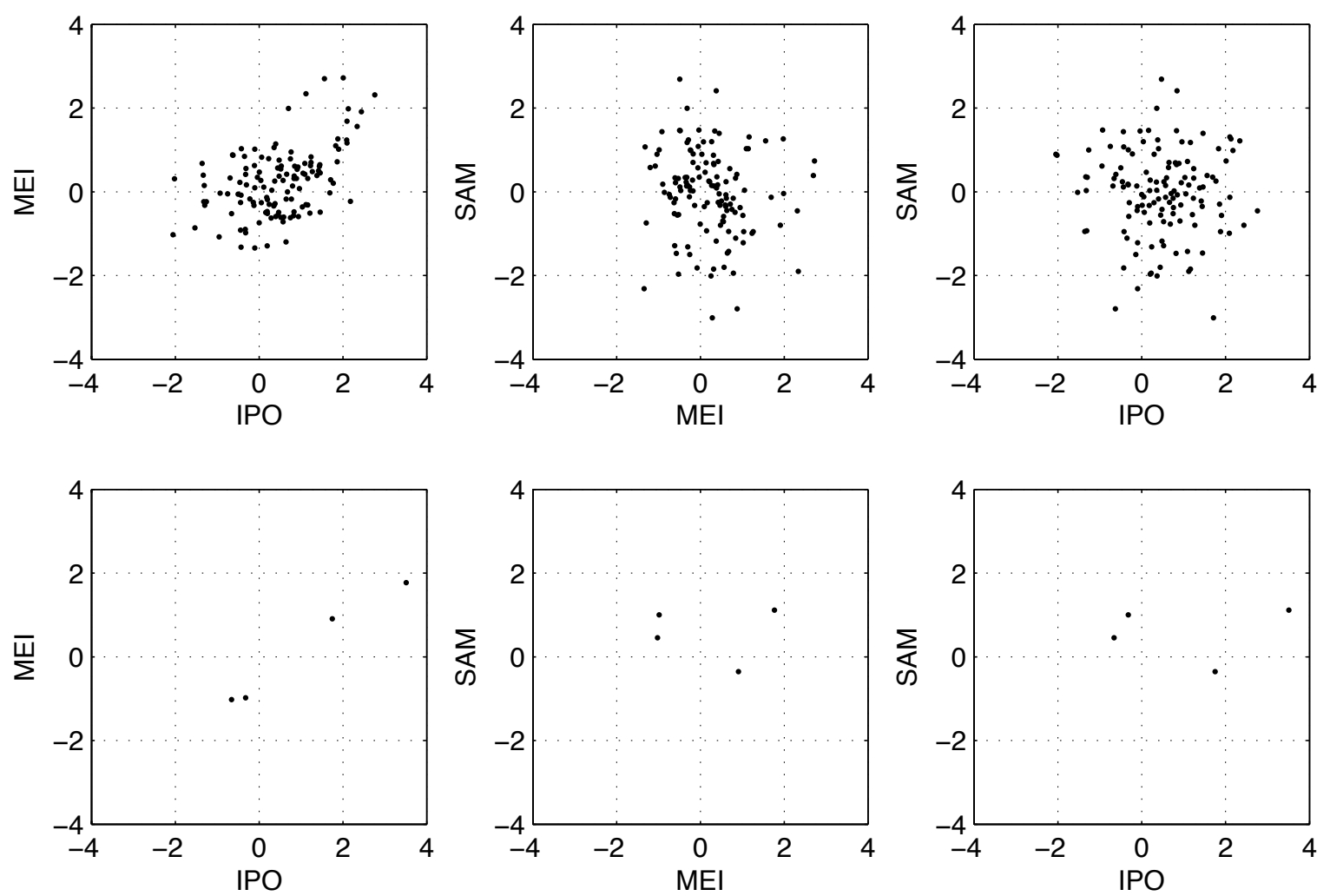

Figure 3.17 Comparing the index value of the different large-scale oscillations (IPO, MEI, and SAM) during extreme events (top row) and droughts (bottom row).

20th century to determine if the characteristics could be correlated to known climate variations, and if these correlations could be used to calculate seasonal probabilities of future heavy precipitation events occurring. The daily regional time series values can be used to put future quantitative precipitation forecast values into the context of the severity of previous extremes.

Heavy precipitation events were identified as those above a 95th percentile threshold. This identified all reported problematic weather events over the recent period, the two historic floods missed in our definition of an extreme consisted of two consecutive weeks slightly below the threshold. A consistent long period time series was used for examining linear changes and climate oscillations within the Waikato River catchment. During this study it was desirable to create a single precipitation time series that represented the total volume of precipitation falling within the Waikato river catchment. To achieve this, spatial and temporal averaging were applied to all stations within the 

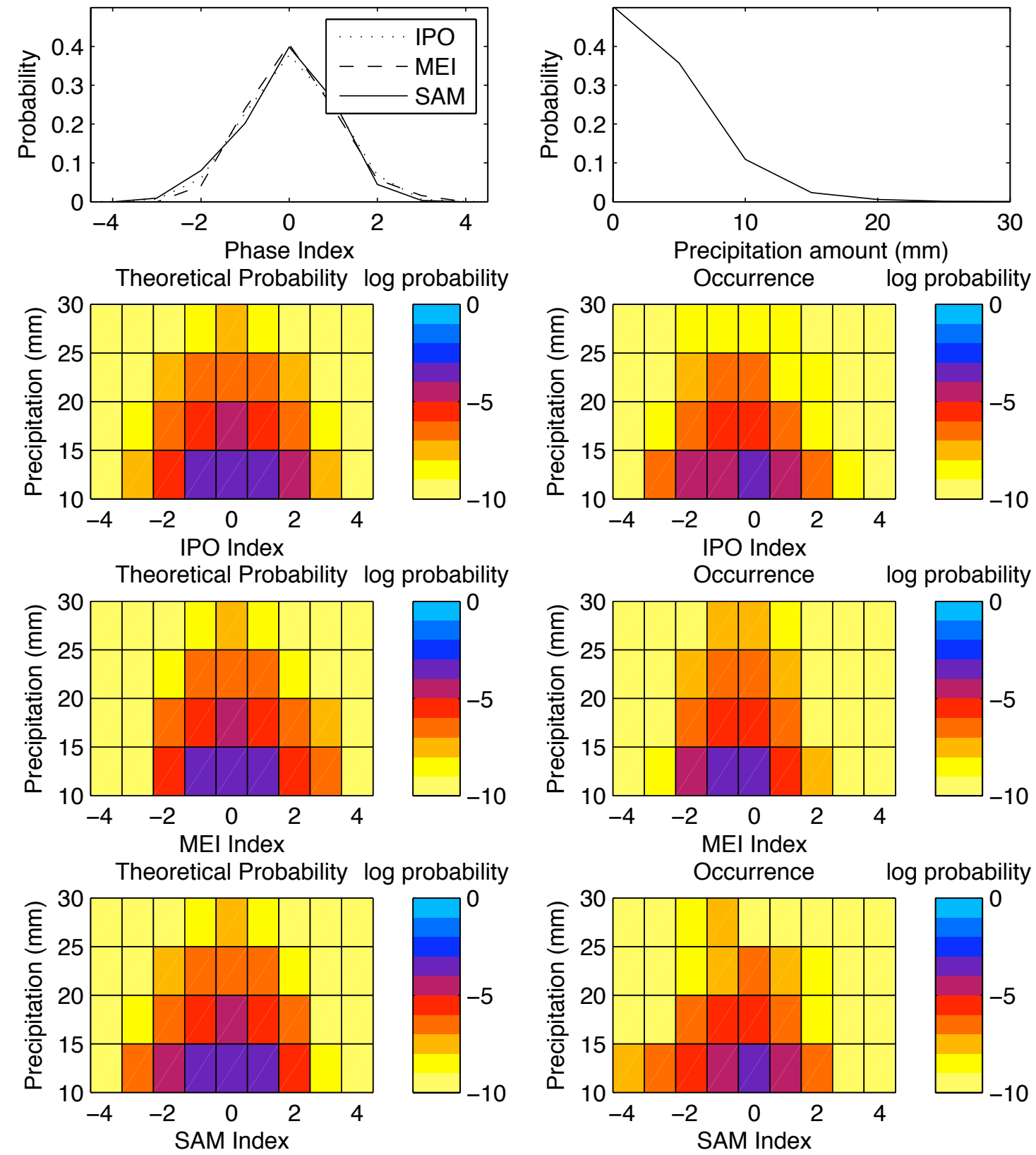

Figure 3.18 The theoretical probability relationship between extreme event occurrence and large-scale circulation indices. The statistical probability of (a) being in a particular phase; (b) receiving a particular volume of precipitation. The left column of subplots shows the theoretical probability and right hand observed occurrence. The second to fourth rows show the probabilities for the IPO; MEI; and SAM index respectively. 
catchment area that contained data for at least 85\% of days between 1996 to 2006 or 50 years of data between 1900 and 2006. Through the development of the time series it was shown that a single station cannot approximate the volume of precipitation occurring within an area the size of this catchment, particularly as only $35 \%$ of the same events were identified from our regional time series and the single station that has been used previously. The discrepancy between the events identified shows that using a regional time series to study extremes will provide more confidence that the results are representative over the catchment area as opposed to a single location that may not be representative.

It has been theorised that future changes in extreme precipitation climatology may possibly be related to an increase in global mean temperature (Trenberth et al., 2003). Whilst this may be true in a globally averaged sense, there is no evidence of it in this regional study. The temperature time series calculated here showed a $1.15^{\circ} \mathrm{C} \pm$ $0.45^{\circ} \mathrm{C}$ increase in temperature over the century. The increase was not smooth and is consistent with values obtained in Salinger et al. (1995) and Zheng et al. (1997) for temperature increases in New Zealand. The regional composite time series used here implies that the increase is widespread across the catchment and not confined to individual locations.

The time series of annual precipitation showed a positive (but statistically insignificant) trend with a $77 \mathrm{~mm}$ increase $(2 \%)$ in the annual precipitation totals over the 20thc̃entury. Griffiths (2007, between 1930 and 2004) calculated a 10\% decrease in the annual precipitation value for the single station (station 2101, Ruakura) that was included in our area integration. This same station recorded a $4 \%$ increase in our study (see Figure 3.10). Neither of these values have statistical significance at the $95 \%$ confidence level and the different values calculated from different time periods suggest that there is an absence of linear variation. Griffiths (2007) also showed that there were increasing trends in the west and decreasing trends in the east. The station 2101 (this study) or Ruakura (Griffiths, 2007) is in the central part of the catchment and recorded the more negative trend while the catchment area as a whole is potentially 
increasing. When this linear increase in the regional time series is compared to individual stations within the area it can be seen through the cumulative summations that it is inappropriate to infer a linear increase.

The variability of drought characteristics and the infrequent nature of these events meant there was insufficient data for useful statistical analysis. All droughts have occurred in summer and there is a suggestion that the frequency of droughts may be decreasing. Increases in drought conditions have been seen in New Zealand regions prone to drought conditions (Mullan et al., 2005) but there is no evidence that drought frequency is changing for this river catchment.

Under our definition of an extreme event there was an insignificant 1.53 events per year increase in the frequency of extreme precipitation event over the time period of the temperature increase. Over this period Salinger et al. (2001) showed an average $2 \%$ decrease in the occurrence of events in New Zealand, but also showed large regional variations and variations associated with the IPO. Their single Waikato station has a trend indistinguishable from zero. The definitions of extremes varied between the studies, which along with the lack of statistical significance could explain the differences.

Using the definition of duration, intensity and total volume applied in this study a number of conclusions have been drawn. The intensity and volume of an event tends to increase as the duration increases. When the intensity is compared to the volume, the same total volume of precipitation can be measured from either a short duration intense event, or a longer duration less intense event. Equal threat could be posed by either event as the same total amount of precipitation will be within the river system at the same time. This is consistent with observations of flooding episodes that have occurred in the Waikato River catchment in the past.

When annual precipitation totals at individual stations were considered, it was evident that linear trends could not account for the variability. The cumulative summation plots for four of the five stations showed that there are non-linear signals in the precipitation trends that are more influential on the time series. Although statisti- 
cally significant widespread global precipitation increases were calculated by Alexander et al. (2006) they were spatially incoherent. Non-significant trends were calculated by Manton et al. (2001), Koning and Franses (2005), and Türkes (1996) in the Southwest Pacific, the Netherlands, and Turkey respectively. The consistency of the precipitation totals suggests that the current dependable water supply is secure within the region in the foreseeable future.

The frequency of occurrence and characteristics of events were examined for a relationship to any of the three prominent climate oscillations. Each of the oscillations, IPO, ENSO, and SAM, is defined by a monthly index. Droughts showed an insignificant preference for positive and neutral index values of all oscillations. The occurrence of heavy precipitation events is uncorrelated to the index values of each oscillation. When the oscillations were compared to each other it can be seen that the IPO and MEI indices correlate to each other in both the occurrence of droughts and heavy precipitation events. However, neither of these oscillations correlates to the SAM index. Despite Gershunov and Cayan (2003) stating that ENSO could be used as a predictor of extreme events in the southwest United States, the lack of correlation seen here means that these oscillations should not be used as a predictor for extreme events in the Waikato River catchment. As extreme events are uncorrelated to large-scale circulations but annual totals are, it may mean that the weather features resulting in extremes have different mechanisms, or are approaching from different directions.

From this study there has been no distinguishable variation in the characteristics or occurrence of heavy precipitation or drought events within the Waikato River catchment since 1900. There has been a measurable increase in temperature, which is consistent with other calculations of mean temperature increases for New Zealand. Global climate models (IPCC, 2007) suggest that in the future the westerly circulation is likely to increase and therefore conditions in New Zealand are more likely to resemble current negative ENSO conditions. While this may not affect the probability of an extreme event occurring, the increase in non-extreme precipitation may mean that the ground is more frequently saturated and extremes are more likely to lead to flooding. 
This study showed that heavy precipitation events have remained consistent over the 20th century. The frequency or characteristics of heavy precipitation events were not related to common circulation changes such as the IPO, ENSO, and SAM, so these should not be used for seasonal prediction of possible extreme events. Therefore it is important to know what meteorological situations are causing these extreme events and how well they can be forecast. 
CHAPTER 4

\section{RECENT EVENTS}

This chapter analyses the characteristics of synoptic weather scenarios that have produced heavy precipitation in the Waikato River catchment, especially those that led to flooding, to identify which characteristics in future forecasts are likely to produce heavy precipitation. From the analysis of all heavy precipitation events that affected the Waikato River catchment over an 11-year period, future forecasts containing similar characteristics can be flagged to indicate that they may have the potential to produce heavy precipitation.

Weather patterns in New Zealand are typical of oceanic mid-latitude locations, with precipitation often being associated with mid-latitude cyclones (Garnier, 1950). Baroclinic mid-latitude cyclones often develop along the Australian eastern land/sea boundary and pass over New Zealand (Sinclair, 1995a; Sinclair, 1995b; Sinclair, 1994; Trenberth, 1991). The precipitation in all cyclones is often enhanced on the coastal side of topographic features, which can protect the leeward catchment during heavy precipitation but also prevent precipitation during a drought. Previous studies of extreme events in the North Island focus on areas outside the catchment (e.g. Munro (2004), Munro (2002), Munro (1998) or Sinclair (1993)) despite the events also impacting the Waikato River catchment.

In recent literature, case studies are normally numerical modelling based (e.g. Falvey and Garreaud (2007), Palecki et al. (2005), and Konrad (2001)) and/or deal only with devastating storms that were poorly predicted (e.g. Chang et al. (2008), Pezza and Simmonds (2005), or Milbrant and Yau (2001)). Sixty-three extreme precipitation 
events that occurred between 1996 and 2007 were individually analysed. Three of the largest events are presented in this chapter as case studies. Geopotential height minima were then tracked through the Tasman Sea to determine what proportions of these mid-latitude cyclones were associated with heavy precipitation.

\subsection{Data}

The data used in this chapter include the daily precipitation observations made at 253 rain gauges in and around the region as described in Chapter 3. The event analysis also used six-hourly analysis maps from the Meteorological Service of New Zealand Ltd, and reanalysis geopotential height data (from the National Centers for Environmental Prediction (NCEP), (Kalnay et al., 1996)). The NCEP reanalysis data extracted were the daily 1800 UTC, 500 and 1000 mb geopotential heights on a 1 x 1 degree grid for the region 140 to $200 \mathrm{E}$ and 20 to $60 \mathrm{~S}$.

\subsection{Recent extreme precipitation events}

Over the 11-year period three drought events were identified and 63 extreme events were identified by the criteria of consecutive days above the 95th percentile in the area-averaged, five-day smoothed time series (these droughts and extreme events are identified in Figure 3.7). Droughts occurred on average once every four years and unsurprisingly, although not statistically significant, all occurred during summer.

On average there are five heavy precipitation events per year, with an average of three consecutive days exceeding the threshold. The average intensity of an event is $13.6 \mathrm{~mm}$ per day and separately the average total volume is $44.8 \mathrm{~mm}$ of precipitation averaged over the entire region per event.

The frequency of occurrence, duration, intensity and volume were examined for seasonal dependence in Figure 4.1. Events were most frequent during spring (SON); however, on average these events were of shorter duration and lower intensity and 

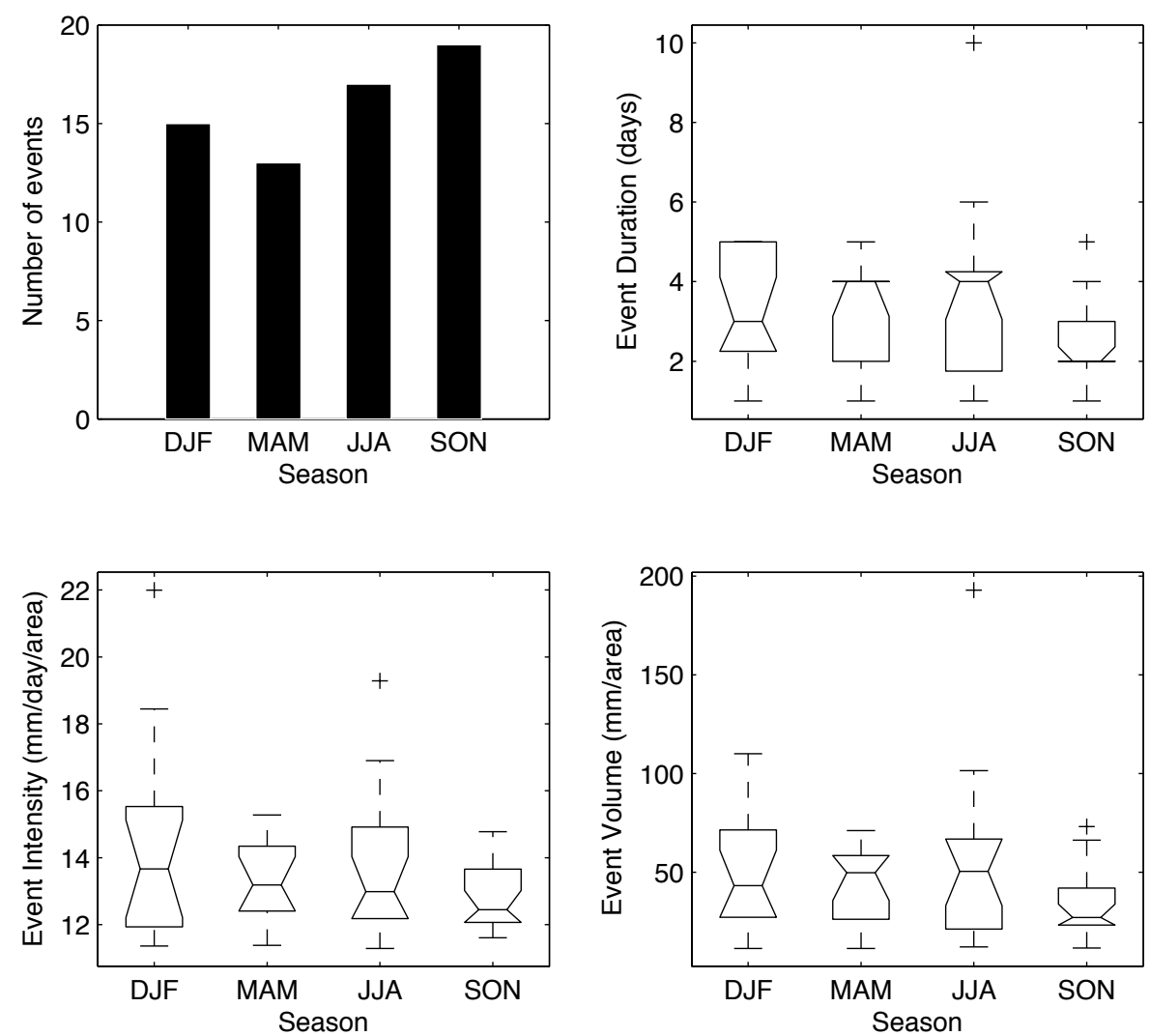

Figure 4.1 Seasonal characteristics of extreme precipitation events. (a) A histogram of event occurrence per season. Seasons are labelled according to the first initial of the months involved. (b) - (d) Show the distributions of event duration, intensity and volume per season through box and whisker plots. The boxes extend from the lower to upper quartiles with notches showing the median to within 5\%, outliers below the 5th and above the 95th percentiles are marked by + signs.

thus spring has lower total volumes than other seasons. The highest intensity events occurred during summer, while typically, the largest volume events occurred in winter. While events were more common in spring, it is actually the high intensity events in summer and long duration events in winter that caused the largest volumes of precipitation in the Waikato.

The 63 events identified in this study were examined in more detail using analysis maps, reanalysis fields, and daily rainfall distribution maps to identify the synoptic weather situation for each event. It is interesting to note that over this period the NewZ̃ealand Meteorological Society independently listed 58 of these events in their list of nationally 'notable weather events' (McGavin, Quarterly Newsletter). Although 
this is not an objective comparison, it gives some degree of confidence that a large proportion of the events identified objectively caused some degree of disruption.

Through the case studies the project aimed to provide details of the types and sizes of synoptic weather features that caused heavy precipitation specifically within the Waikato River catchment. However, some events could not be attributed to a single synoptic scale weather feature (referred to as 'feature') and therefore each feature was separately classified. Table 4.1 summarises all 63 events, and three of these events were selected as case studies for further examination in this chapter.

The author found no suitable objective methods in the literature for defining the synoptic scale weather system causing the heavy precipitation so a subjective set of criteria was used. A major study by Kidson (1994) and (2000) attempted to objectively classify synoptic-scale weather in New Zealand and this classification has been used in a number of subsequent studies (e.g. Griffiths (2007)). However, this objective classification scheme tends to obscure the meteorologically active aspects of synoptic features, such as the cyclogenesis associated with precipitation in New Zealand, and was therefore not used here.

In this study, geopotential height fields and analysis maps were used to categorise the features in each event. The identified features were each defined as one of the following:

- C: a 'cyclone' with a closed minimum in the $1000 \mathrm{hPa}$ geopotential height field, and its associated frontal bands (this includes both mid-latitude and subtropical cyclones);

- T: an 'ex-tropical cyclone', i.e. any cyclone that tracked back to a named tropical cyclone, and its associated frontal bands;

- F: a 'front' that is detached from any closed minimum within the map area.

Features are counted separately if they appear to have developed independently, although this does not negate the possibility that the features are interconnected on a 
larger scale. Frontal bands are associated with a cyclone if the shape of the front curves around or terminates at the pressure minimum.

The origin of each feature was defined by where the first minimum appeared in the reanalysis $1000 \mathrm{mb}$ geopotential height field (Figure 4.2), or where the majority of the frontal line was in the first analysis map in which it appeared. Origin boundaries

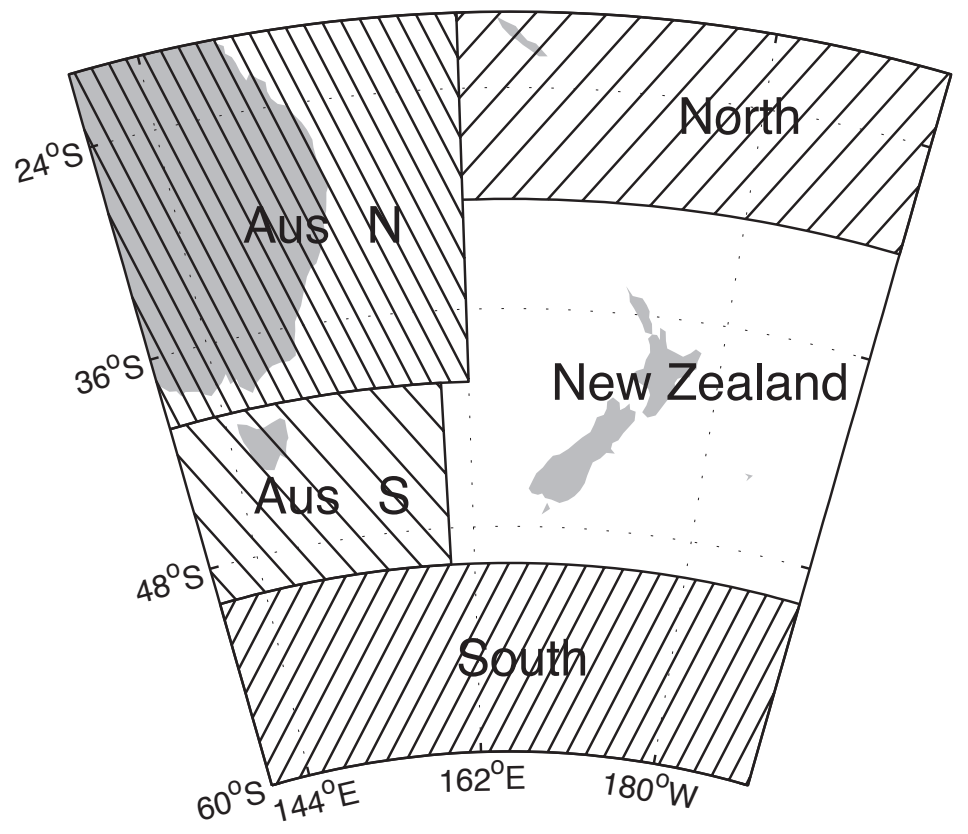

Figure 4.2 Origin boundary classifications for features between $22^{\circ} \mathrm{S}$ and $60^{\circ} \mathrm{S}$ and $140^{\circ} \mathrm{E}$ to $170^{\circ} \mathrm{W}$ : North between $165^{\circ} \mathrm{E}$ and $160^{\circ} \mathrm{E}$ and north of $30^{\circ} \mathrm{S}$, South is south of $50^{\circ} \mathrm{S}$, Australianorth is west of $165^{\circ} \mathrm{E}$ and north of $37^{\circ} \mathrm{S}$, Australia-south is west of $155^{\circ} \mathrm{E}$ and between $37^{\circ} \mathrm{S}$ and $50^{\circ} \mathrm{S}$, and New Zealand all locations outside the aforementioned boundaries.

were selected after all of the origins were located on the map, and the boundaries were subjectively placed in low density areas that grouped features that were likely to be similar; e.g. the eastern coastline of Australia that was identified as an area of cyclogenesis by Sinclair (1995a). Table 4.1 lists the features for each of the 63 events, and Table 4.2 summarises the synoptic features identified and where each originated. 


\section{Table 4.1}

The 63 extreme precipitation events between 1996 and 2007, identified by the first date (UTC) when the percentile was exceeded. The duration (Dur.) and volume (Vol.) and MEI index value are as defined in Chapter 3, then for each identified feature the type $(T)$ and origin $(O)$ is given. The types are: $C$ is a cyclone, $T$ an ex-tropical cyclone, and $F$ a front, as described in the text. The origins are as shown in Figure 4.2, although North, South, and New Zealand have been abbreviated.

\begin{tabular}{|c|c|c|c|c|c|c|c|c|c|c|c|}
\hline \multirow{2}{*}{$\begin{array}{c}\text { Date } \\
\mathrm{dd} / \mathrm{mm} / \text { yyyy }\end{array}$} & \multirow{2}{*}{$\begin{array}{l}\text { Dur. } \\
\text { (days) }\end{array}$} & \multirow{2}{*}{$\begin{array}{l}\text { Vol. } \\
\text { mm }\end{array}$} & \multirow[t]{2}{*}{ MEI } & \multicolumn{2}{|c|}{ Feature 1} & \multicolumn{2}{|c|}{ Feature 2} & \multicolumn{2}{|c|}{ Feature 3} & \multicolumn{2}{|c|}{ Feature 4} \\
\hline & & & & $\mathrm{T}$ & $\mathrm{O}$ & $\mathrm{T}$ & $\mathrm{O}$ & $\mathrm{T}$ & $\mathrm{O}$ & $\mathrm{T}$ & $\mathrm{O}$ \\
\hline $13 / 1 / 1996$ & 3 & 37.6 & -0.6 & & Aus-N & & & & & & \\
\hline $7 / 2 / 1996$ & 3 & 34.9 & -0.6 & $\mathrm{C}$ & $\mathrm{NZ}$ & $\mathrm{C}$ & Aus-S & & & & \\
\hline $20 / 2 / 1996$ & 1 & 11.8 & -0.6 & $\mathrm{C}$ & Aus-N & $\mathrm{F}$ & S & & & & \\
\hline $20 / 4 / 1996$ & 4 & 49.7 & -0.5 & & Aus-S & & & & & & \\
\hline $21 / 5 / 1996$ & 4 & 57.0 & -0.2 & & Aus-N & & & & & & \\
\hline $16 / 7 / 1996$ & 5 & 69.4 & -0.2 & & Aus-S & $\mathrm{C}$ & Aus-N & & & & \\
\hline $20 / 8 / 1996$ & 4 & 50.4 & -0.3 & & Aus-N & & & & & & \\
\hline $4 / 9$ / 1996 & 5 & 66.3 & -0.3 & & Aus-N & & & & & & \\
\hline $9 / 9 / 1996$ & 2 & 23.3 & -0.3 & & Aus-S & & & & & & \\
\hline $31 / 12 / 1996$ & 1 & 11.4 & -0.3 & $\mathrm{~T}$ & $\mathrm{~N}$ & & & & & & \\
\hline $23 / 5 / 1997$ & 4 & 58.4 & +1.1 & & Aus-N & & & & & & \\
\hline $1 / 6 / 1997$ & 4 & 61.1 & +2.3 & $\mathrm{C}$ & $\mathrm{S}$ & $\mathrm{C}$ & $\mathrm{N}$ & & & & \\
\hline $25 / 9 / 1997$ & 1 & 12.9 & +2.8 & & Aus-N & $\mathrm{C}$ & Aus-S & & & & \\
\hline $14 / 11 / 1997$ & 1 & 12.4 & +2.3 & $\mathrm{C}$ & $\mathrm{S}$ & & & & & & \\
\hline $23 / 2 / 1998$ & 3 & 41.0 & +2.7 & & Aus-S & & & & & & \\
\hline $12 / 3 / 1998$ & 1 & 12.2 & +2.7 & $\mathrm{C}$ & $\mathrm{NZ}$ & F & $\mathrm{S}$ & & & & \\
\hline $25 / 5 / 1998$ & 2 & 25.9 & +2.0 & & Aus-S & $\mathrm{C}$ & Aus-S & & & & \\
\hline $15 / 6 / 1998$ & 1 & 12.5 & +1.1 & $\mathrm{C}$ & $\mathrm{N}$ & $\mathrm{C}$ & Aus-S & & & & \\
\hline $14 / 7 / 1998$ & 10 & 192.9 & +0.3 & $\mathrm{~F}$ & $\mathrm{NZ}$ & $\mathrm{C}$ & Aus-N & $\mathrm{C}$ & NZ & $\mathrm{C}$ & Aus-N \\
\hline $10 / 8 / 1998$ & 4 & 51.6 & -0.2 & & Aus-N & & & & & & \\
\hline $12 / 10 / 1998$ & 3 & 36.6 & -0.8 & $\mathrm{C}$ & Aus-N & & & & & & \\
\hline
\end{tabular}

continued on next page 


\begin{tabular}{|c|c|c|c|c|c|c|c|c|c|c|}
\hline & & Vol. & MEI & & ature 1 & & ature 2 & & ture 3 & Feature 4 \\
\hline $\mathrm{dd} / \mathrm{mm} /$ yyyy & (days) & $\mathrm{mm}$ & & $\mathrm{T}$ & $\mathrm{O}$ & $\mathrm{T}$ & $\mathrm{O}$ & $\mathrm{T}$ & $\mathrm{O}$ & $\mathrm{T} \quad \mathrm{O}$ \\
\hline $2 / 12 / 1998$ & 2 & 24.6 & -0.9 & $\mathrm{C}$ & $\mathrm{N}$ & $\mathrm{C}$ & $\mathrm{NZ}$ & & & \\
\hline $16 / 1 / 1999$ & 5 & 79.5 & -1.0 & $\mathrm{C}$ & NZ & $\mathrm{F}$ & $\mathrm{NZ}$ & & & \\
\hline $14 / 6$ / 1999 & 1 & 13.0 & -0.4 & $\mathrm{C}$ & Aus-N & $\mathrm{C}$ & S & & & \\
\hline $17 / 7 / 1999$ & 4 & 56.2 & -0.5 & & Aus-N & & & & & \\
\hline $15 / 9 / 1999$ & 2 & 25.0 & -0.8 & $\mathrm{C}$ & Aus-N & & & & & \\
\hline $6 / 11 / 1999$ & 1 & 12.4 & -1.0 & $\mathrm{C}$ & $\mathrm{N}$ & $\mathrm{C}$ & Aus-N & & & \\
\hline 10/11/ 1999 & 4 & 54.8 & -1.0 & & Aus-N & & & & & \\
\hline 27/11/ 1999 & 3 & 37.1 & -1.0 & $\mathrm{C}$ & $\mathrm{NZ}$ & & & & & \\
\hline $9 / 4 / 2000$ & 4 & 58.4 & -0.3 & & Aus-N & $\mathrm{C}$ & $\mathrm{N}$ & & & \\
\hline $3 / 6 / 2000$ & 5 & 71.1 & -0.3 & $\mathrm{C}$ & Aus-S & $\mathrm{C}$ & $\mathrm{NZ}$ & & & \\
\hline $7 / 9 / 2000$ & 2 & 23.8 & -0.2 & S & Aus-S & $\mathrm{C}$ & Aus-S & & & \\
\hline $2 / 10 / 2000$ & 3 & 42.0 & -0.3 & $\mathrm{C}$ & S & & & & & \\
\hline $29 / 12 / 2000$ & 2 & 23.3 & -0.6 & $\mathrm{C}$ & S & & & & & \\
\hline $11 / 2 / 2001$ & 5 & 72.3 & -0.6 & $\mathrm{C}$ & $\mathrm{N}$ & & & & & \\
\hline $3 / 5 / 2001$ & 2 & 26.3 & +0.2 & $\mathrm{C}$ & $\mathrm{NZ}$ & $\mathrm{C}$ & $\mathrm{N}$ & & & \\
\hline $16 / 7 / 2001$ & 4 & 57.9 & -0.0 & & Aus-S & $\mathrm{C}$ & S & & & \\
\hline $30 / 10 / 2001$ & 1 & 11.6 & -0.3 & $\mathrm{C}$ & Aus-S & $\mathrm{C}$ & S & & & \\
\hline $22 / 11 / 2001$ & 3 & 36.1 & -0.2 & & Aus-N & & & & & \\
\hline $8 / 12 / 2001$ & 4 & 57.7 & -0.0 & & Aus-N & & Aus-N & & & \\
\hline $18 / 6 / 2002$ & 2 & 24.3 & +0.8 & $\mathrm{C}$ & $\mathrm{N}$ & & & & & \\
\hline $4 / 7 / 2002$ & 6 & 101.4 & +0.6 & & Aus-S & & & & & \\
\hline $9 / 1 / 2003$ & 5 & 68.0 & +1.2 & $\mathrm{C}$ & $\mathrm{N}$ & & & & & \\
\hline $21 / 5 / 2003$ & 3 & 37.0 & +0.0 & & Aus-S & & & & & \\
\hline $6 / 6 / 2003$ & 1 & 12.2 & -0.0 & & Aus-N & $\mathrm{F}$ & $\mathrm{S}$ & & & \\
\hline $16 / 6 / 2003$ & 2 & 23.9 & -0.0 & $\mathrm{C}$ & $\mathrm{S}$ & $\mathrm{C}$ & $\mathrm{NZ}$ & $\mathrm{C}$ & $\mathrm{NZ}$ & \\
\hline $3 / 9 / 2003$ & 2 & 27.0 & +0.5 & $\mathrm{C}$ & $\mathrm{S}$ & & & & & \\
\hline $28 / 9 / 2003$ & 2 & 24.3 & +0.5 & $\mathrm{C}$ & Aus-N & $\mathrm{C}$ & S & & Aus-N & \\
\hline
\end{tabular}




\begin{tabular}{|c|c|c|c|c|c|c|c|c|c|}
\hline \multirow{2}{*}{$\begin{array}{c}\text { Date } \\
\mathrm{dd} / \mathrm{mm} / \text { yyyy }\end{array}$} & \multirow{2}{*}{$\begin{array}{l}\text { Dur. } \\
\text { (days) }\end{array}$} & \multirow{2}{*}{$\begin{array}{l}\text { Vol. } \\
\mathrm{mm}\end{array}$} & \multirow[t]{2}{*}{ MEI } & \multicolumn{2}{|c|}{ Feature 1} & \multicolumn{2}{|c|}{ Feature 2} & Feature 3 & Feature 4 \\
\hline & & & & $\mathrm{T}$ & $\mathrm{O}$ & $\mathrm{T}$ & $\mathrm{O}$ & $\mathrm{T} \quad \mathrm{O}$ & $\mathrm{T} \quad \mathrm{O}$ \\
\hline $25 / 11 / 2003$ & 4 & 59.1 & +0.5 & $\mathrm{C}$ & Aus-N & $\mathrm{C}$ & Aus-N & & \\
\hline $1 / 2 / 2004$ & 5 & 92.2 & +0.4 & $\mathrm{C}$ & Aus-N & & & & \\
\hline $28 / 2 / 2004$ & 5 & 110.0 & +0.4 & $\mathrm{C}$ & Aus-N & $\mathrm{T}$ & $\mathrm{N}$ & & \\
\hline $3 / 5 / 2004$ & 1 & 11.4 & +0.4 & $\mathrm{C}$ & Aus-N & $\mathrm{C}$ & S & & \\
\hline $18 / 6 / 2004$ & 5 & 81.2 & +0.3 & $\mathrm{C}$ & Aus-S & $\mathrm{C}$ & S & & \\
\hline $6 / 8 / 2004$ & 1 & 13.1 & +0.6 & $\mathrm{C}$ & Aus-S & & & & \\
\hline $29 / 5 / 2005$ & 3 & 39.6 & +0.7 & $\mathrm{C}$ & S & $\mathrm{C}$ & NZ & & \\
\hline $16 / 7 / 2005$ & 2 & 24.0 & +0.4 & & Aus-N & & & & \\
\hline $17 / 9 / 2005$ & 5 & 73.1 & -0.2 & $\mathrm{C}$ & Aus-N & $\mathrm{C}$ & Aus-S & & \\
\hline $3 / 10 / 2005$ & 2 & 23.2 & -0.2 & & Aus-N & & & & \\
\hline $9 / 10 / 2005$ & 3 & 42.0 & -0.2 & $\mathrm{C}$ & S & & Aus-S & & \\
\hline $17 / 12 / 2005$ & 3 & 43.2 & -0.5 & $\mathrm{C}$ & $\mathrm{NZ}$ & $\mathrm{C}$ & $\mathrm{NZ}$ & & \\
\hline $24 / 1 / 2006$ & 4 & 68.9 & -0.4 & $\mathrm{C}$ & $\mathrm{N}$ & $\mathrm{C}$ & S & & \\
\hline $27 / 4 / 2006$ & 4 & 53.4 & -0.6 & $\mathrm{C}$ & $\mathrm{N}$ & & & & \\
\hline $6 / 8 / 2006$ & 5 & 80.8 & +0.8 & $\mathrm{C}$ & S & & & & \\
\hline
\end{tabular}

\begin{tabular}{|c|c|c|c|c|}
\hline & $\mathrm{C}$ & $\mathrm{T}$ & $\mathrm{F}$ & Total \\
\hline New Zealand & 13 & 0 & 2 & 15 \\
\hline North & 11 & 2 & 0 & 13 \\
\hline South & 16 & 0 & 3 & 19 \\
\hline Aus-N & 32 & 0 & 0 & 32 \\
\hline Aus-S & 20 & 0 & 0 & 20 \\
\hline Total & 92 & 2 & 5 & 99 \\
\hline
\end{tabular}

Table 4.2

Summary of synoptic scale features and feature origins occurring during statistically identified events. Features and origins are defined in the text.

A total of 99 features were identified for the 63 events. One event consisted of a sequence of four features and a further two events had a sequence of three features. Thirty-two events (51\%) were attributed to a single synoptic feature. Ninety-two of the features identified were cyclones, which have a known association with precipitation in New Zealand (Garnier, 1950). The majority of mid-latitude cyclones originated near the east coast of Australia, an area of recognised high cyclogenesis (see Sinclair 
(1995a), (1995b), (1994), or Trenberth (1991) for details). There were also two extropical cyclones, and six frontal bands not associated with a visible cyclone. All cyclones had cold cores when they passed over New Zealand, although some may have initially developed with a warm core, especially those that developed at lower latitudes.

Although there was no evidence (Chapter 3) that extreme precipitation event occurrence or magnitude was associated with the ENSO phenomenon, this phenomenon has been associated with higher frequency cyclonic development during El Niño and blocking during La Niña. Therefore the MEI index number during each of the events in Table 4.1 was noted. Although there was a La Niña phase during 1997/1998 the rest of the 11-year period was relatively neutral. The largest events (by volume) and the events containing the most features all occurred during a neutral phase so the features or sequences of events could not be correlated to the MEI index. However, this data set does not represent the complete range of MEI index values.

\subsection{Case studies}

Although all 63 cases were analysed, three selected events are discussed here in more detailed. These are:

- July 1998: the second largest flood ever recorded in the Waikato River catchment;

- February 2004: contained the largest daily precipitation value recorded during the 11-year period;

- January 2006: was the largest event that could be included in the modelling study (due to availability of global model data, see Chapter 6, where this event is also a case study), this event produced more than $90 \%$ of the average January precipitation in two days.

Coincidentally, and fortunately, these three events contained most of the typical feature types and origins described earlier. For each event the daily and smoothed regional 
precipitation time series are plotted, and selected MetService analysis maps, geopotential height maps, and precipitation distribution maps are also included to show the development and track of identified features.

\subsubsection{July 1998 - a complex succession of cyclones}

As mentioned, the July 1998 flood was one of the largest on record in the Waikato (Roper, 2001; Munro, 1998). This event had precipitation levels in excess of the 95th percentile on ten consecutive days (Figure 4.3), including two daily peaks that exceeded the 99th percentile of daily precipitation. During this event three intense mid-latitude

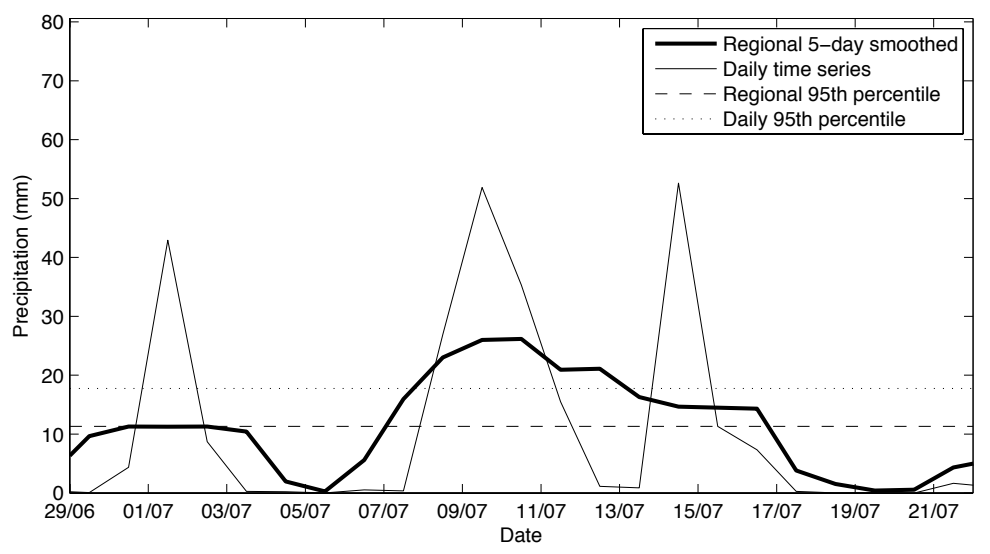

Figure 4.3 The regional area-averaged time series (both daily and five-day smoothed, and the 95th percentiles for each) for the multi-feature event of July 1998.

cyclones and a disassociated front passed over New Zealand within a week.

The first feature of this event was a slow-moving cold front in a baroclinic trough (Figures 4.4a-b and 4.6a) producing heavy precipitation on 9 July 1998 (times and dates are UTC). The front was prevented from continuing eastward by a blocking high and remained over New Zealand until the arrival of the second feature.

The second feature was a mid-latitude cyclone that began cyclogenesis east of Australia at $\sim 37^{\circ} \mathrm{S}$ on 9 July (Figures $4.4 \mathrm{a}-\mathrm{c}$ and $4.5 \mathrm{a}-\mathrm{c}$ ). There were distinct pressure minima at both the 1000 and 500 mb levels until after this feature had travelled past 

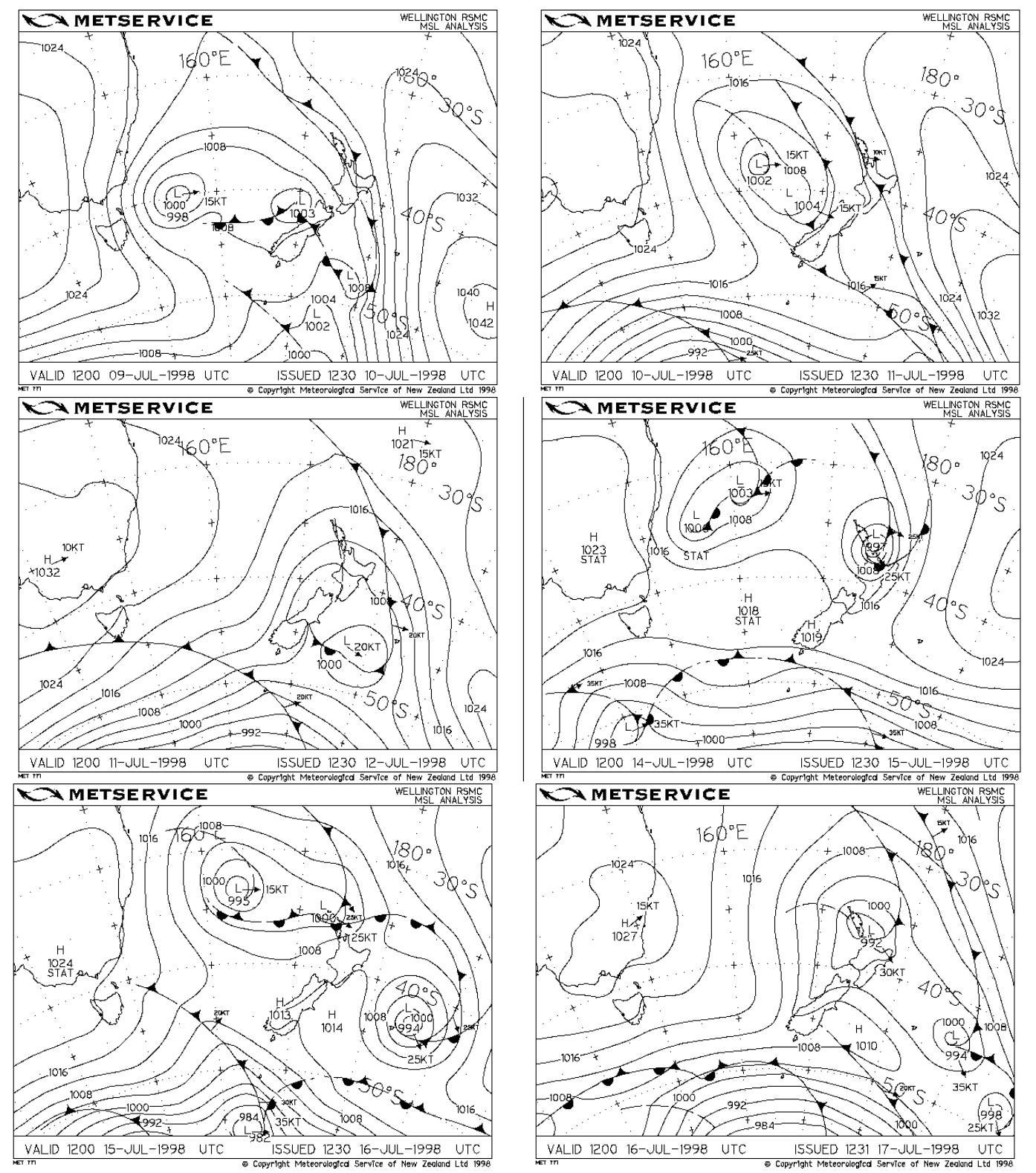

Figure 4.4 A selection of MetService analysis maps for the multi-feature event of July 1998. These maps show the development of these features for the following dates: (a) 9, (b) 10, (c) 11, (d) 14, (e) 15 and (f) 16 July 1998. A vertical bar in map sequences signifies a discontinuity in the sequence.

New Zealand on 10 July producing the widespread heavy precipitation seen in Figure 4.6b.

The third feature was a shallower mid-latitude cyclone that developed over the North Island of New Zealand on 14 July. Figure 4.5(d) shows that there was no minimum present in the $500 \mathrm{mb}$ geopotential height field although the field contained 

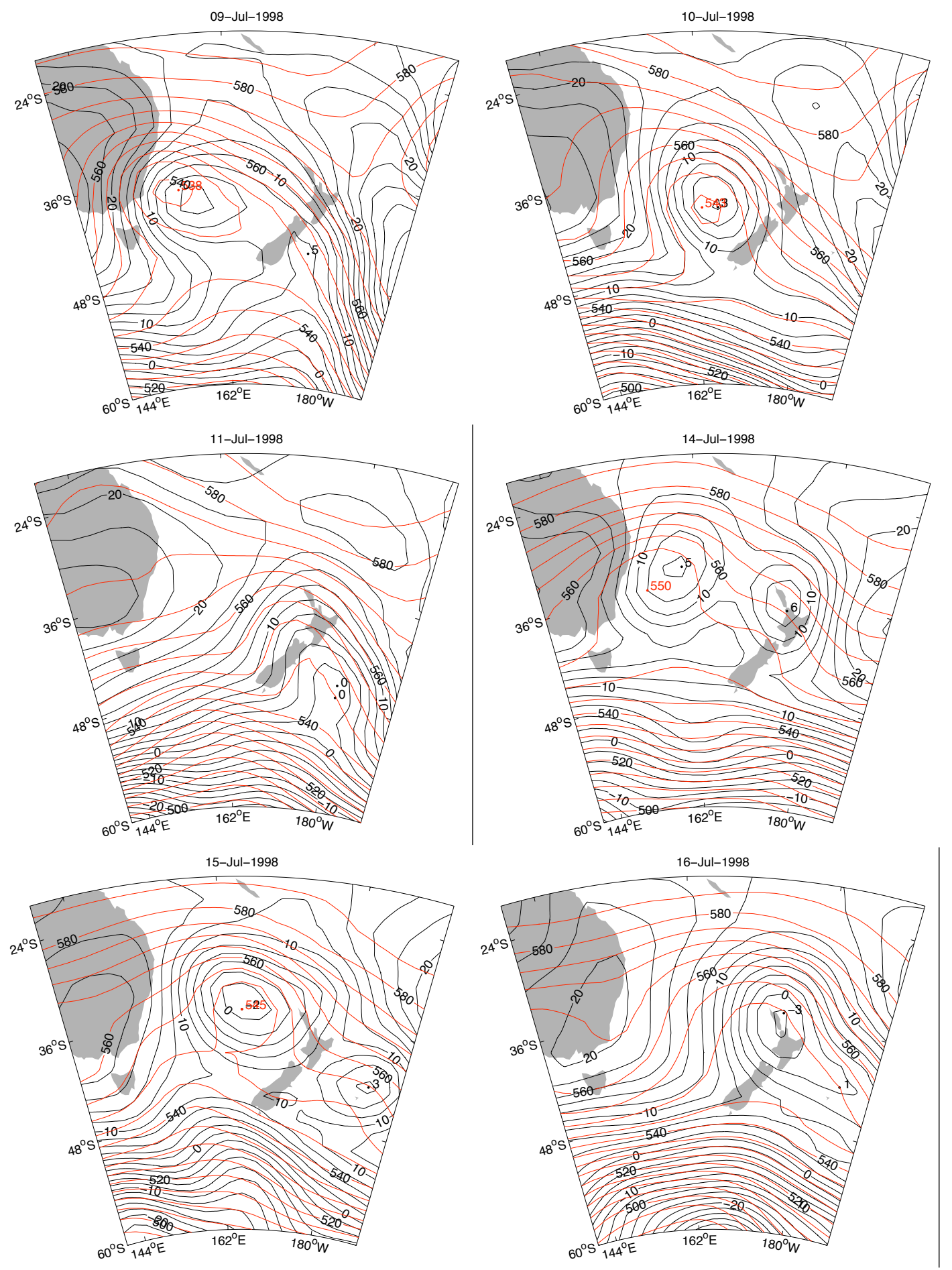

Figure 4.5 A selection of geopotential height maps for the multi-feature event of July 1998. These maps show the development of these features at both the $1000 \mathrm{mb}$ (black contours) and the $500 \mathrm{mb}$ (red contours) height fields for the following dates: (a) 9, (b) 10, (c) 11, (d) 14, (e) 15 and (f) 16 July 1998. 

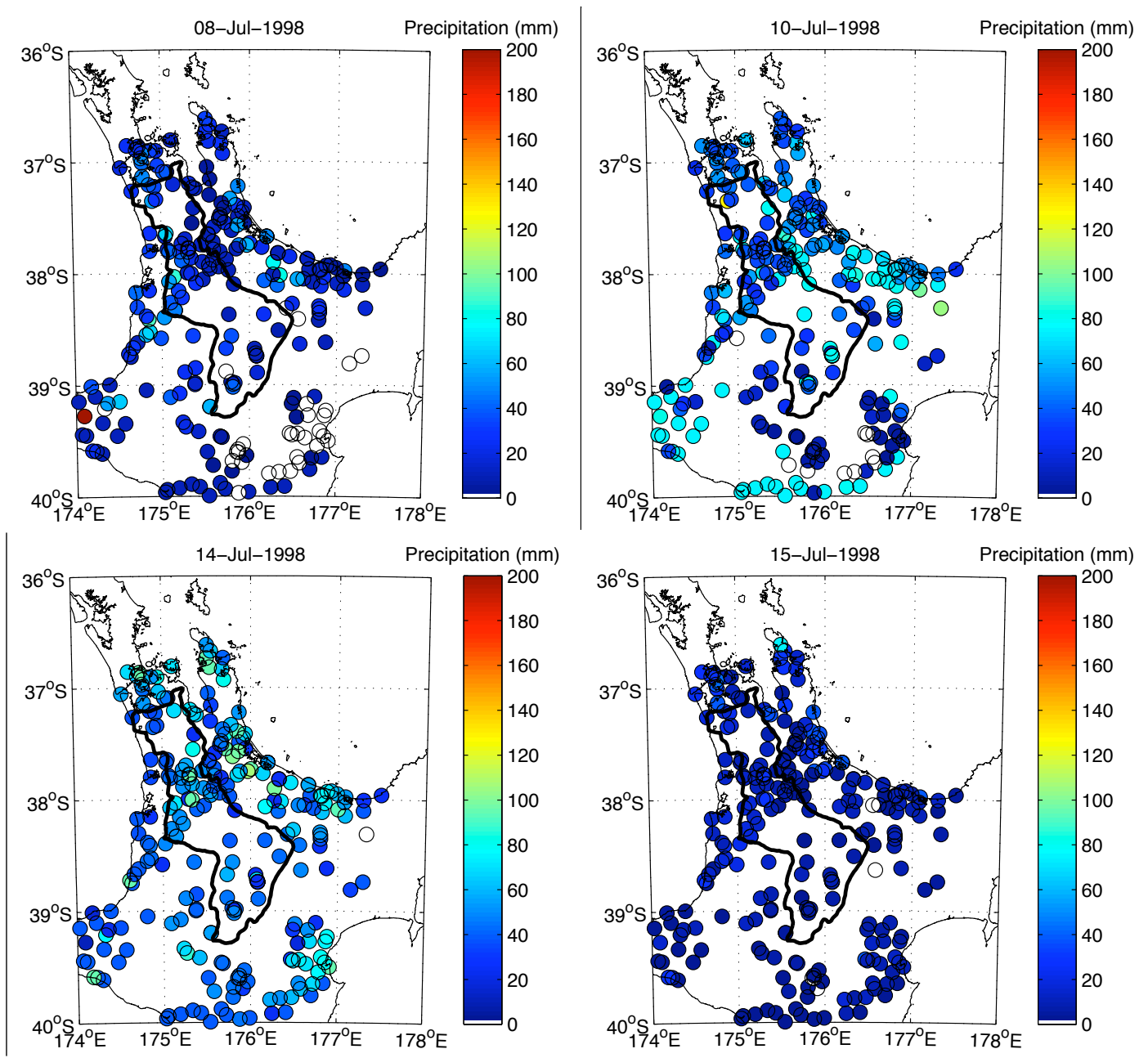

Figure 4.6 A selection of daily precipitation distribution maps for the multi-feature event of July 1998. The daily distributions are shown here for the 8, 10, 14 and 15 July 1998 and coincide with the dates of heaviest precipitation as shown in Figure 4.3.

a disturbance above the surface minimum. This feature passed quickly but produced widespread heavy precipitation (Figure 4.6c) especially northeast of the Waikato River catchment.

The final feature in this sequence was another mid-latitude cyclone that began cyclogenesis east of Australia at $\sim 32^{\circ} \mathrm{S}$ on 14 July (Figures $4.4 \mathrm{~d}-\mathrm{f}$ and $4.5 \mathrm{~d}-\mathrm{f}$ ). This cyclone deepened as it passed over the Tasman Sea; however, only light widespread precipitation was observed when it passed over New Zealand on 15 July (Figure 4.6d). The upper level minimum was also much shallower during this feature.

The flood was caused by the rapid succession of cyclones passing over New Zealand 
associated with enhanced baroclinicity. These features also suggest that the depth of the $500 \mathrm{mb}$ minimum is more significant than the surface minimum in determining the quantity of precipitation that will occur under a mid-latitude cyclone.

This event resulted in large-scale flooding as the catchment was already saturated from a separate intense cyclone that occurred on 1 and 2 July, only a week prior and was only slightly below the 95th percentile threshold (see Figure 4.3).

A similar sequence of cyclones occurred again in July 2008 in another period of enhanced baroclinicity, this again resulted in widespread flooding (however this was outside the data collection period and not included in this study).

\subsubsection{February 2004 - coincident ex-tropical and mid-latitude cyclone}

The February 2004 flood was another one of the largest floods on record, and was caused by the simultaneous arrival of the frontal bands from a mid-latitude cyclone and an ex-tropical cyclone on $28 \tilde{F}$ ebruary. The heaviest precipitation occurred on 28 February (Figure 4.7), and was the heaviest daily regional (area-averaged un-smoothed time series) precipitation recorded during the 11 years.

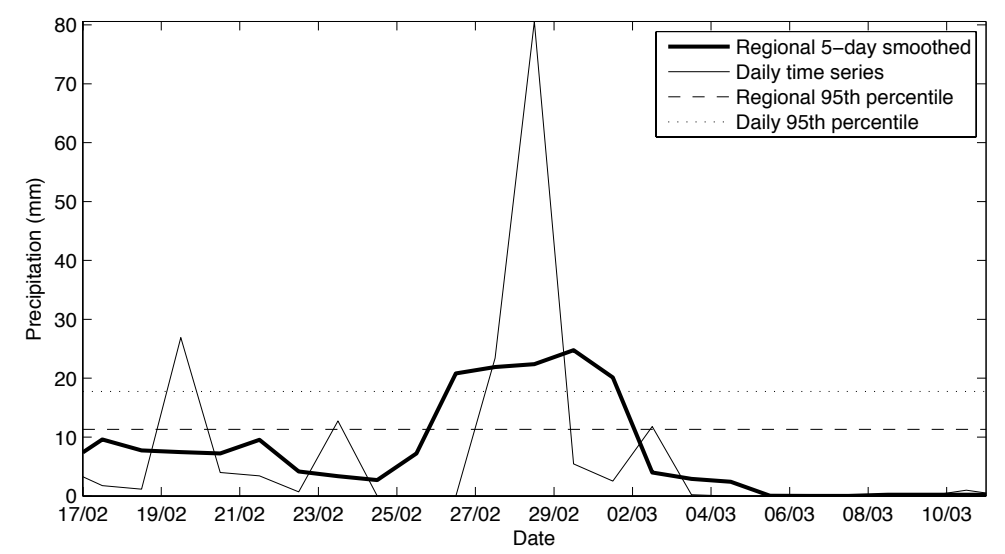

Figure 4.7 The regional area-averaged time series (both daily and five-day smoothed, and the 95th percentiles for each) for the ex-tropical cyclone event of February 2004.

A mid-latitude cyclone developed east of Australia at $34^{\circ} \mathrm{S}$ on 26 February (Figures $4.8 \mathrm{a}$ and $4.9 \mathrm{a})$. The surface minimum intensified as the cyclone traversed the Tasman 


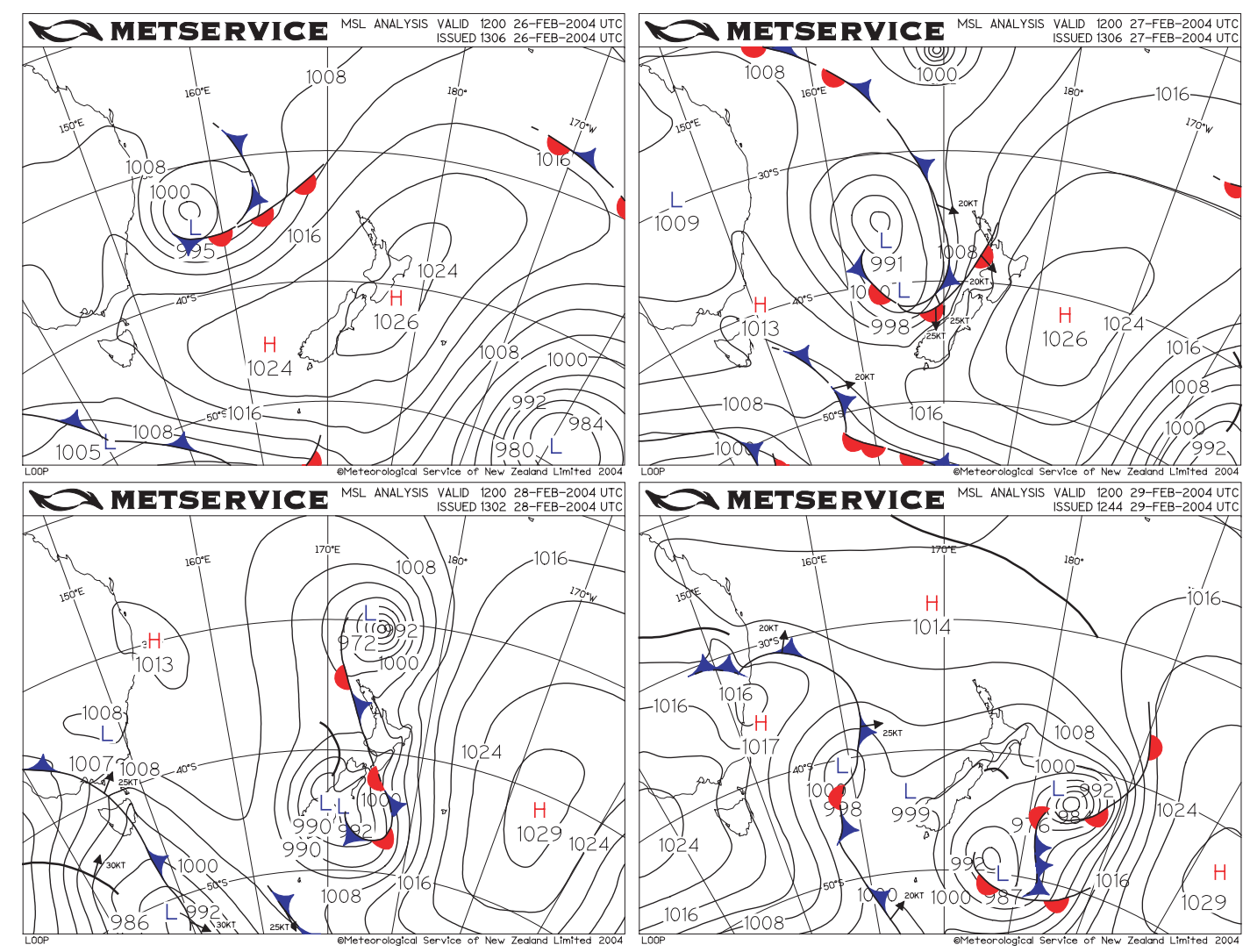

Figure 4.8 A selection of MetService analysis maps for the ex-tropical cyclone event of February 2004. These maps show the development of these features for the following dates: (a) 26, (b) 27, (c) 28 and (d) 29 February 2004.

Sea, although the $500 \mathrm{mb}$ level minimum abated. The cyclone tracked over the central South Island on 28 February (Figures $4.8 \mathrm{c}$ and $4.9 \mathrm{c}$ ).

On 27 February ex-tropical cyclone 'Ivy' appeared in the north with a cold core (Figures $4.8 \mathrm{~b}$ and $4.9 \mathrm{~b}$ ). Over the next 24 hours this cyclone travelled south-southeast and the upper level minimum also abated. Precipitation from both of these features arrived in the Waikato River catchment on 28 February (Figure 4.10b) creating the largest daily precipitation total observed in the 11-year period.

Either of these cyclones would have produced large precipitation totals but the simultaneous arrival and interaction of the two cyclones produced the record high precipitation total. This event occurred a week after the southern half of the North Island had been devastated by another storm which also saturated the Waikato River catchment. Therefore flooding resulted from record high precipitation occurring over 

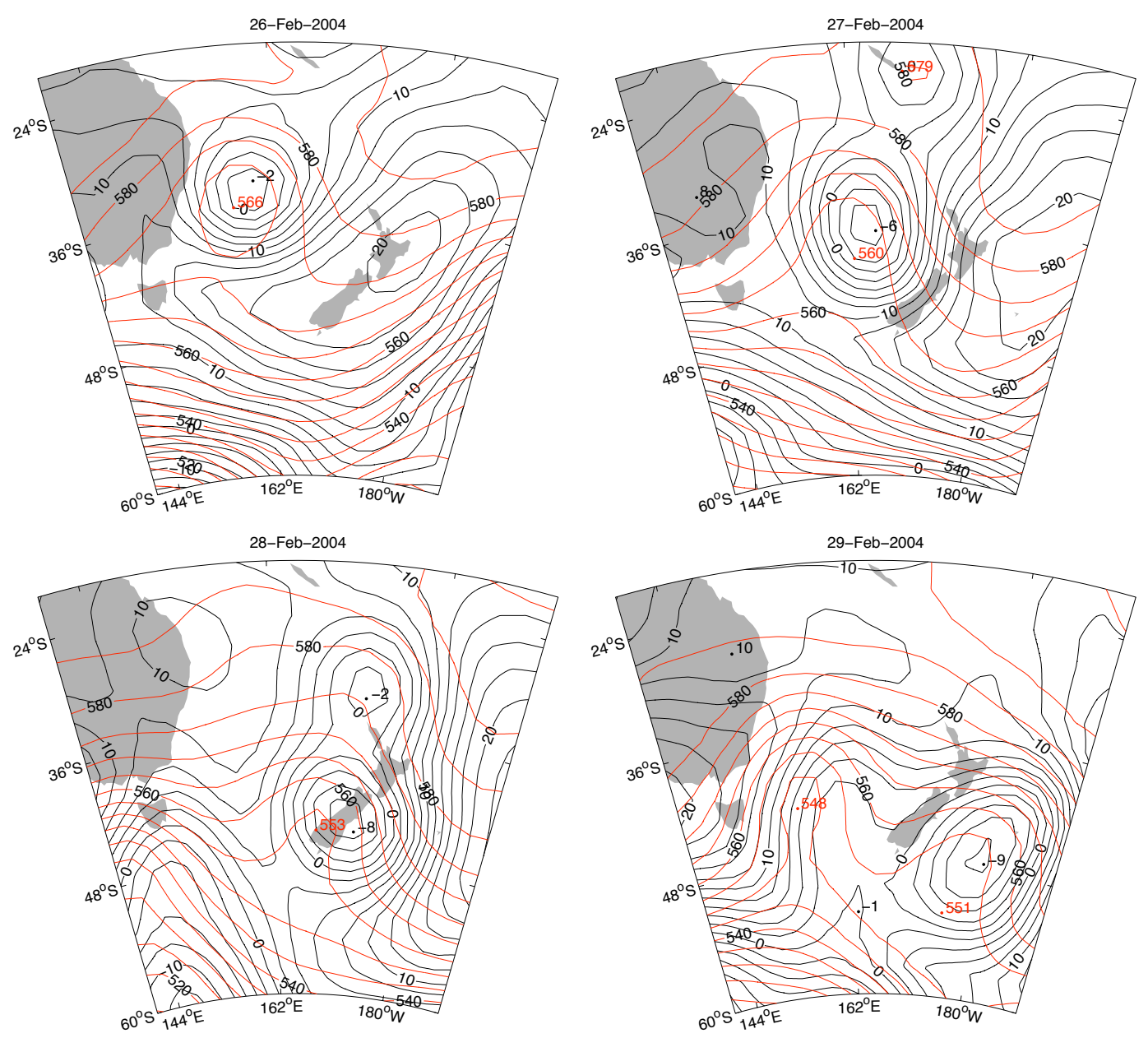

Figure 4.9 A selection of geopotential height maps for the ex-tropical cyclone event of February 2004. These maps show the development of these features at both the $1000 \mathrm{mb}$ (black contours) and the $500 \mathrm{mb}$ (red contours) height fields for the following dates: (a) 26, (b) 27, (c) 28 and (d) 29 February 2004.

an already saturated catchment.

\subsubsection{January 2006 - subtropical and Southern Ocean cyclones}

The impact of the flooding from this final case study was much less than in the previous cases, but more than $90 \%$ of the average January precipitation fell in the two-day period. The rapid succession of a subtropical cyclone and the frontal bands of a Southern Ocean mid-latitude cyclone produced the largest daily precipitation peak in the two years for which GFS model initialisation data was available (data set described in Chapter 5). This event is also used as a case study in the mesoscale simulation in 

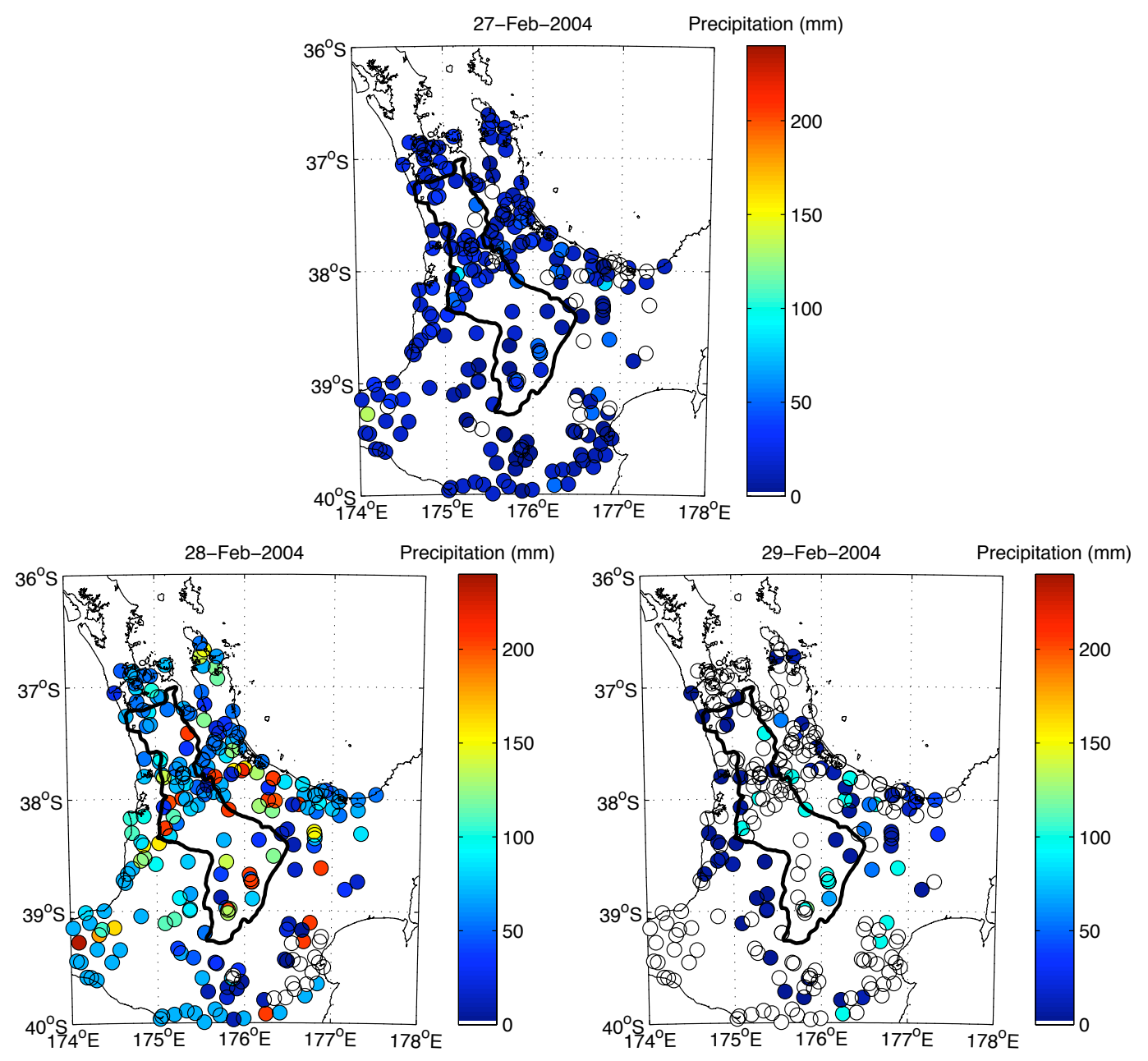

Figure 4.10 A selection of daily precipitation distribution maps for the ex-tropical cyclone event of February 2004. The daily distributions are shown here for (a) 27, (b) 28, and (c) 29 February 2004 and coincide with the dates of heaviest precipitation as shown in Figure 4.7.

Chapter 6 .

A subtropical cyclone appeared to the north-northwest of New Zealand on 22 January and reached the northern tip of the North Island on 24 January (Figures 6.15a-b and 4.12a-c). A large blocking high to the east of New Zealand prevented this cyclone from continuing southeast. The heaviest precipitation in this event occurred under the cold front associated with this feature on 24 January (Figure 4.13b).

A Southern Ocean mid-latitude cyclone is present in the southwest corner of the analysis map for 1200 UTC 23 January (Figure 6.15a). This quickly traversed south of the map extent but the associated cold front passed through the Tasman Sea, where 


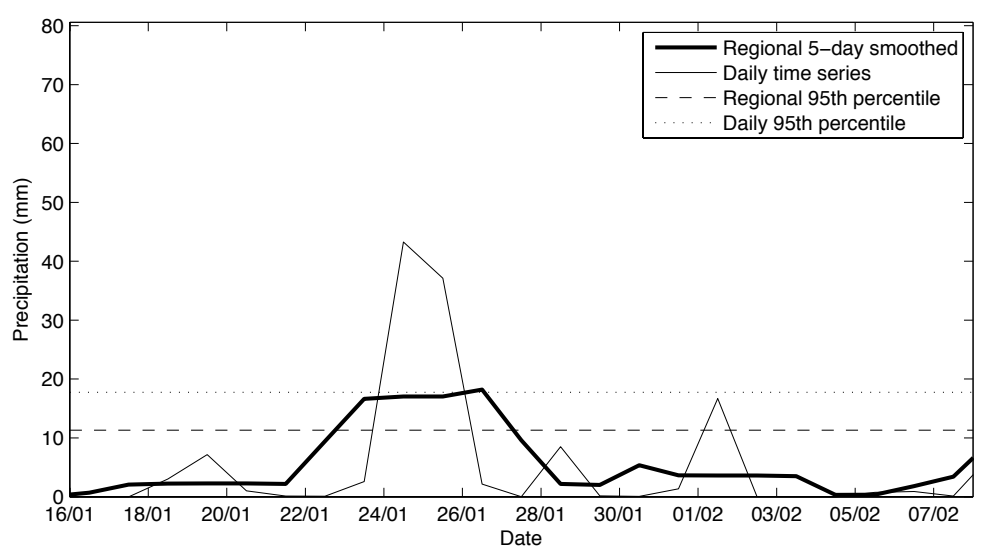

Figure 4.11 The regional area-averaged time series (both daily and five-day smoothed, and the 95th percentiles for each) for the cyclone of northern origin in January 2006.
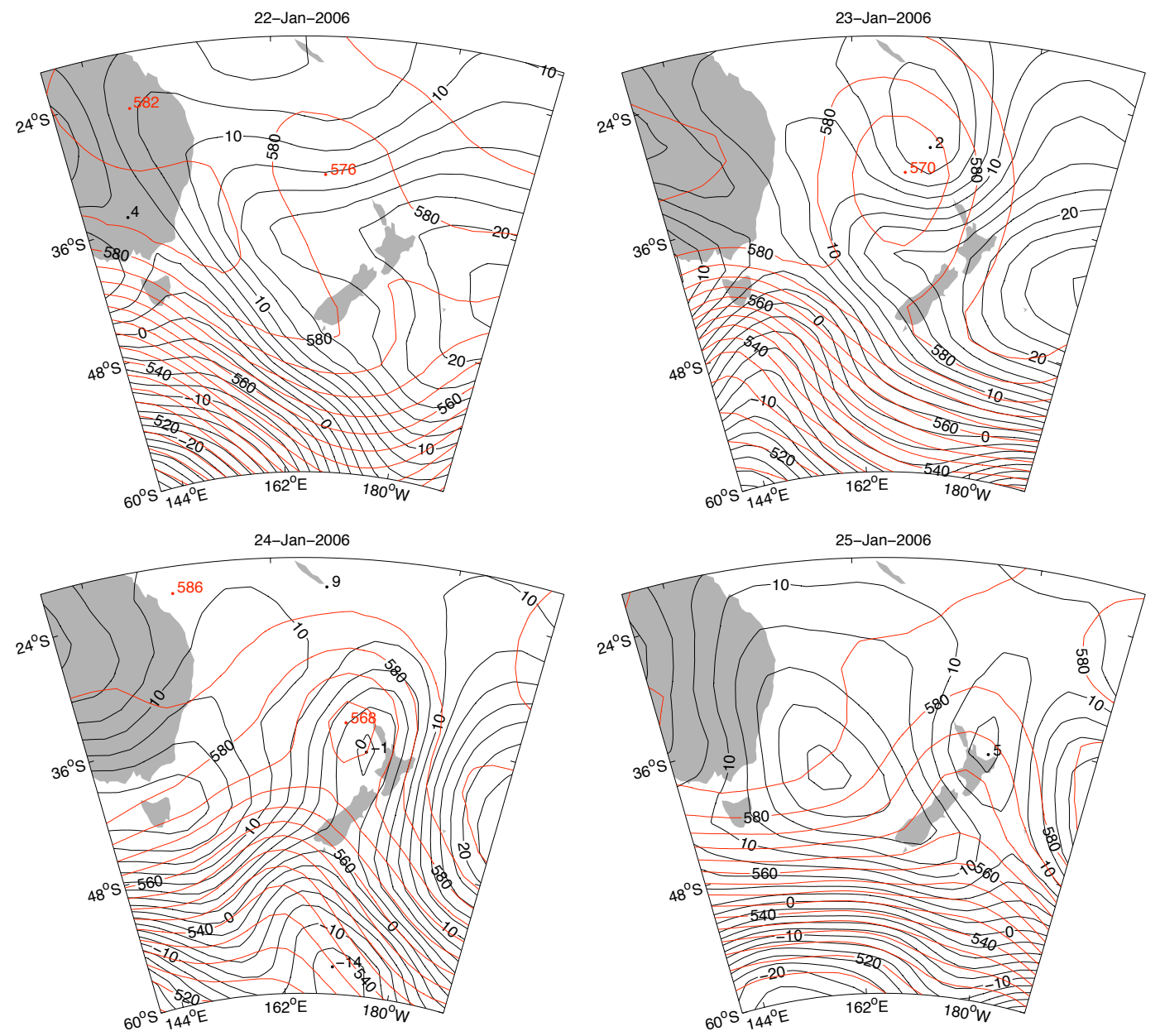

Figure 4.12 A selection of geopotential height maps for the cyclone of northern origin in January 2006. These maps show the development of this feature at both the $1000 \mathrm{mb}$ (black contours) and the $500 \mathrm{mb}$ (red contours) height fields for the following dates: (a) 22, (b) 23, (c) 24 and (d) 25 January 2006. 

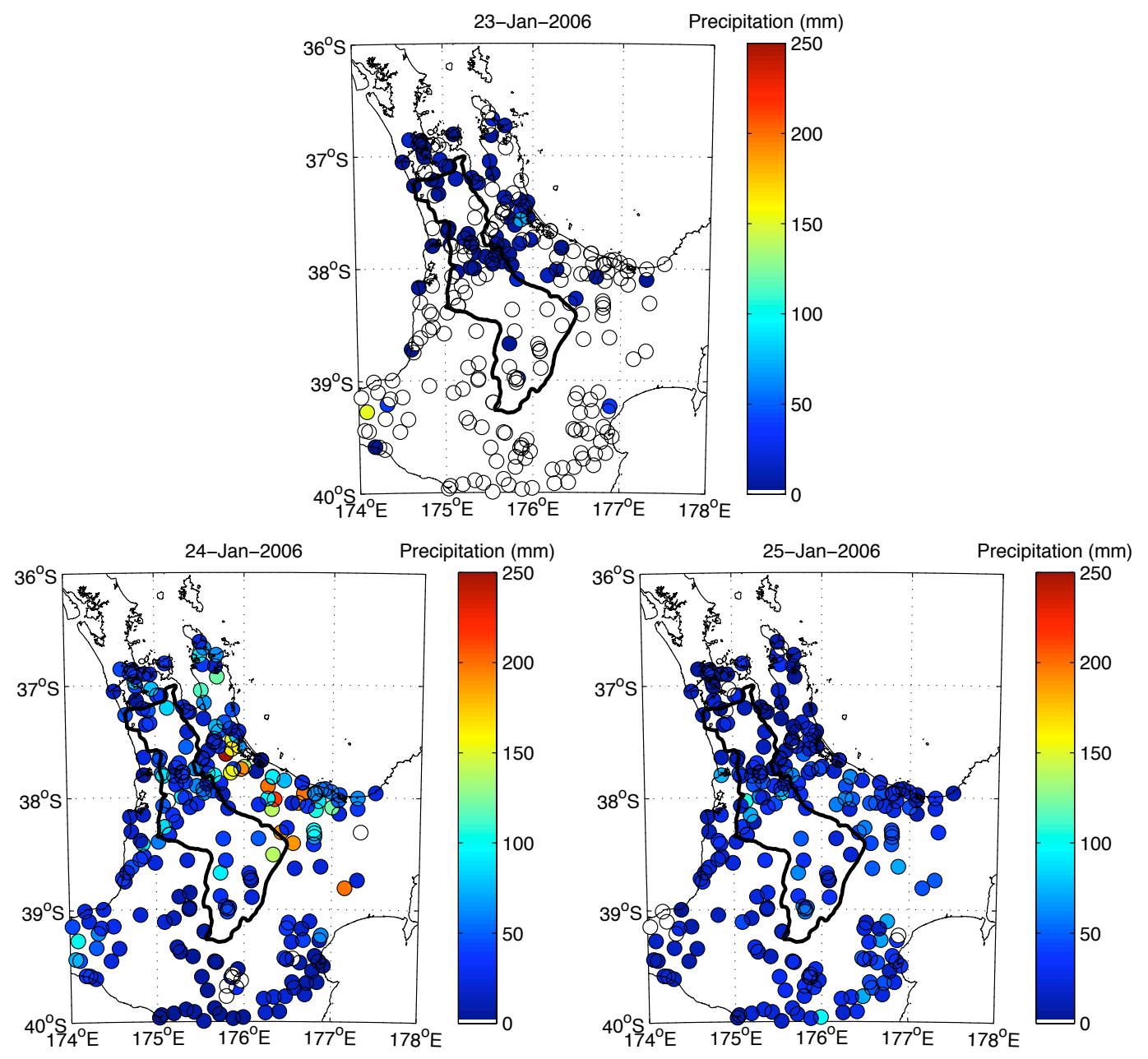

Figure 4.13 A selection of daily precipitation distribution maps for the cyclone of northern origin in January 2006. The daily distribution are shown here for (a) 23, (b) 24, (c) 25 January 2006 and coincide with the dates of heaviest precipitation as shown in Figure 4.11.

it interacted with the subtropical cyclone that had stalled over the North Island. The warm subtropical air mass from the first feature became trapped by this front and produced widespread convective precipitation on 25 January (Figure 4.13c).

This event resulted in two consecutive days of heavy precipitation exceeding the daily 99th percentile (Figure 4.11). Both flash flooding and surface flooding occurred during this event, but the consequences could have been more severe if the catchment had been saturated prior to the event. 


\subsubsection{Common features}

From the 63 events identified and the case studies presented here there are a number of commonalities to the heavy precipitation events. These features are summarised here and will be further examined in some of the numerical simulations presented in Chapter 6.

\section{- Cyclones originating near Australia}

The most common features $(57 \%)$ are the cold core mid-latitude cyclones that develop near the east coast of Australia and are predominantly baroclinically driven, undergoing cyclogenesis along the Australian coastline. The surface minima develop below upper troughs but in nearly every case, the $500 \mathrm{mb}$ upper level developed a closed geopotential height minimum. These cyclones form to the east of the Australian land mass and travel east. Cyclone development frequently continues into the Tasman Sea, although there can be a large variation in the lifetime of the cyclone which in turn causes significant differences in the duration and intensity of the precipitation. Mature cyclone centres frequently pass over the northern South Island with their associated cold fronts bringing precipitation to the Waikato River catchment as happened in both the July 1998 and February 2004 case studies.

\section{- Cyclones originating in the Southern Ocean}

Sixteen percent of features were cyclones that had developed and tracked through the Southern Ocean. These originate poleward of $50^{\circ} \mathrm{S}$, are very deep, and contain long cold fronts that pass over the whole of New Zealand, as in the January 2006 event. The heaviest precipitation usually occurs along western coastlines. These features are associated with troughs at both upper and lower levels, and often occur in a sequence with secondary cyclones that develop in the outer reaches of the trough.

\section{- Cyclones originating in the north}


Subtropical cyclones of northern origin can travel south to New Zealand. These cyclones may be travelling southeast or southwest and it is often the cold front that produces precipitation in the Waikato River catchment as seen in the case study of January 2006. In such cases the Waikato is likely to receive intense precipitation for a short period, although larger volumes of precipitation will fall in the Coromandel and Bay of Plenty.

\section{- Ex-tropical cyclones}

On average approximately once a year an ex-tropical cyclone affects New Zealand. Over this 11-year period only two such ex-tropical cyclones caused heavy precipitation in the Waikato River catchment. In both cases the cyclones developed a cold core before reaching New Zealand (as in the case of February 2004), and brought widespread heavy precipitation for a short period.

\section{- Blocking highs}

If a blocking high (Trenberth and Mo, 1985) is present to the east of New Zealand, the westward migration of cyclones of any origin can be hindered. Some cyclones stall in the Tasman Sea and continue to deepen producing more fronts or more intense precipitation within the frontal zone. However, if the cyclone stalls over the land mass then it often decays, producing widespread persistent light precipitation.

\section{- Cyclone sequences}

Nearly half of the events were associated with a period of enhanced baroclinic activity resulting in more than one feature passing over the area in relatively quick succession. In these cases it was the combined precipitation from the sequence of features that caused the event in the Waikato River catchment. In some cases the features arrived together, as in the case study for February 2004, or multiple phases of baroclinicity passed over the region in quick succession with a series of fast-moving cyclones as in July 1998. These sequences contain more features and are more common in winter $(60 \%$ of winter events contain multiple features 
compared to $40 \%$ of events during summer) due to the seasonal variation in general circulation. Sequences are also the cause of nearly every flood in the region. The two largest floods during the period (flood size is measured by peak discharge in the river (Roper, 2001)), were attributed to a sequence of events.

Where a cyclone develops will determine the types of air masses that are involved, e.g. warm tropical air, or cold southern air. The distance from the origin of the cyclone and New Zealand will influence its developmental stage, i.e. cyclones that originate near Australia will be more mature than a cyclone that develops near the New Zealand coastline and therefore has had more time to accumulate water vapour. The track of any cyclone will also dictate the direction of approach of the associated frontal bands, and this will determine the distribution of precipitation within the catchment.

\subsection{Storm tracks}

Ninety-five percent of features causing heavy precipitation in the Waikato River catchment were cyclones with distinct pressure minima. Although it is well known that precipitation in New Zealand is associated with cyclones, de Lisle (1967) noted that these features occur in the New Zealand area on average once every six to seven days. Obviously not every cyclone produces heavy precipitation, and this tracking study was designed to determine the proportion of tracks that originate in and around the Tasman Sea that coincide with the occurrence of heavy precipitation in the Waikato River catchment.

Common tracking methods, such as that developed by Sinclair (1994) use vorticity fields to track mid-latitude cyclones. Vorticity minima are more common and do not always develop into cyclones, so they could identify many more cyclones than seen in forecast maps. The preference for tracking from vorticity minima in many studies is that this method has less latitude dependency and therefore can track cyclones into the higher latitudes. Despite this, this study tracks geopotential height minima because it is simpler, will have less 'false start' cyclones, and is more comparable to analysis maps 
and forecast charts issued to the public, it was also the parameter used for storm tracks in Pezza and Ambrizzi (2003). Disassociated fronts and Southern Ocean mid-latitude cyclones are not represented in this tracking study but they only account for a small proportion of features, and would have to be a very significant feature to cause heavy precipitation in the Waikato (i.e. they should be identified in forecasts).

To track the pressure minima, ten years (1996-2005) of NCEP reanalysis (Kalnay et al., 1996) $1000 \mathrm{mb}$ geopotential height data at one-degree resolution were used. The data were interpolated using a biharmonic spline interpolation (Sandwell, 1987) to a $0.1 \times 0.1$ degree grid for higher resolution before minima were identified. Details of the tracking method are presented here as the method was specially designed for this particular study.

The first step in the tracking method was to identify all the minima in the geopotential height field. Then for each minimum, $\mathrm{Z}_{i j}$, the depth of the minimum was calculated as the difference between the minimum and the surrounding area (12 grid points either side) by the equation:

$$
\operatorname{depth}\left(Z_{i j}\right)=\frac{1}{12^{2}} \sum_{q=-12}^{12} \sum_{w=-12}^{12} Z_{i-q, j-w}-Z_{i, j}
$$

Therefore with a list of the time, longitude, latitude, and depth of each minimum a tracking system was developed that assigned each minimum to a track. The first unassigned minimum in the list was assigned a track number ( ${ }_{t}$ in the following equations), then for each minimum $\left({ }_{m}\right)$ in the following time step the 'difference' was calculated:

$$
\text { difference }=\sqrt{\text { distance }^{2}+\text { angle }^{2}+\text { magnitude }^{2}}
$$

Where:

$$
\text { distance }=\sqrt{\left(\operatorname{lon}_{t}-\operatorname{lon}_{m}\right)^{2}+\left(\text { lat }_{t}-\text { lat }_{m}\right)^{2}}
$$




$$
\begin{aligned}
& \text { angle }=\operatorname{atan}\left(\left(\operatorname{lon}_{t}-\operatorname{lon}_{m}\right),\left(\text { lat }_{t}-\text { lat }_{m}\right)\right)-\operatorname{atan}\left(\left(\text { lon }_{t}-\operatorname{lon}_{t-1}\right),\left(\text { lat }_{t}-\text { lat }_{t-1}\right)\right) \\
& \text { magnitude }=\frac{\left(\text { depth }_{t}-\text { dept }_{m}\right)}{4}
\end{aligned}
$$

Then the minimum with the smallest difference was also assigned to the track if the distance was less than five degrees. This minimum is now the comparison minimum for the next time step and the process is repeated. When no minimum met the requirement the track was terminated and a new track was started from the first unassigned minimum in the updated list.

The direction of travel (or 'angle') in the above equation was added to the difference equation to prevent the tracking method reversing direction when a second minimum followed behind. This was included when it was noticed that the closest minima (distance-wise) was not always the continuation of the current track; this occurred in cases where the cyclone split into two minima or in times of high baroclinicity where cyclones travelled in quick succession. In the first time step of a track the angle difference was defaulted to zero. The magnitude of the cyclone was also added to the function to resolve the track when the situation became complicated and multiple minima were identified for a single cyclone. This magnitude was down-weighted (by a factor of four; Equation 4.5) in the difference calculation as the magnitude was an order of magnitude larger than the other parameters. Further weighting of the parameters was unnecessary as it did not improve the tracking method.

Once all minima were assigned to a 'track', the additional criteria of traversing more than two degrees during its lifetime and passing within five degrees of New Zealand were applied to identify the storm tracks. The first requirement removed permanent stationary lows, which occur over the Southern Alps of New Zealand, and tracks that consisted of a single minimum. The second requirement limited tracks to those that passed near New Zealand and which therefore could be reasonably expected to impact the Waikato River catchment. Although long frontal bands from cyclones that never passed within the designated five degree range are known to affect the catchment, especially during Southern Ocean cyclones, the majority of these storms will have 
little impact.

The passage of a cyclone tended to produce a peak in daily precipitation values, and so all daily regional time series peaks exceeding the 90th percentile were identified for this section of the study. The threshold was reduced to the 90th percentile to increase the number of events selected. There were 106 identified precipitation peaks. Our tracking method resulted in 653 tracks passing near New Zealand during the tenyear period. Only 68 of these tracks coincided with the 106 heavy precipitation events observed in the Waikato River catchment, these are deemed to be 'matched'. This equates to $64 \%$ of the 106 identified events, approximately the same as the proportion of features identified in the case studies to originate in the area. Ten percent of all identified tracks coincided with heavy precipitation within the Waikato area and these tracks are shown in Figure 4.14. Many of the cyclones moved to the southeast, which

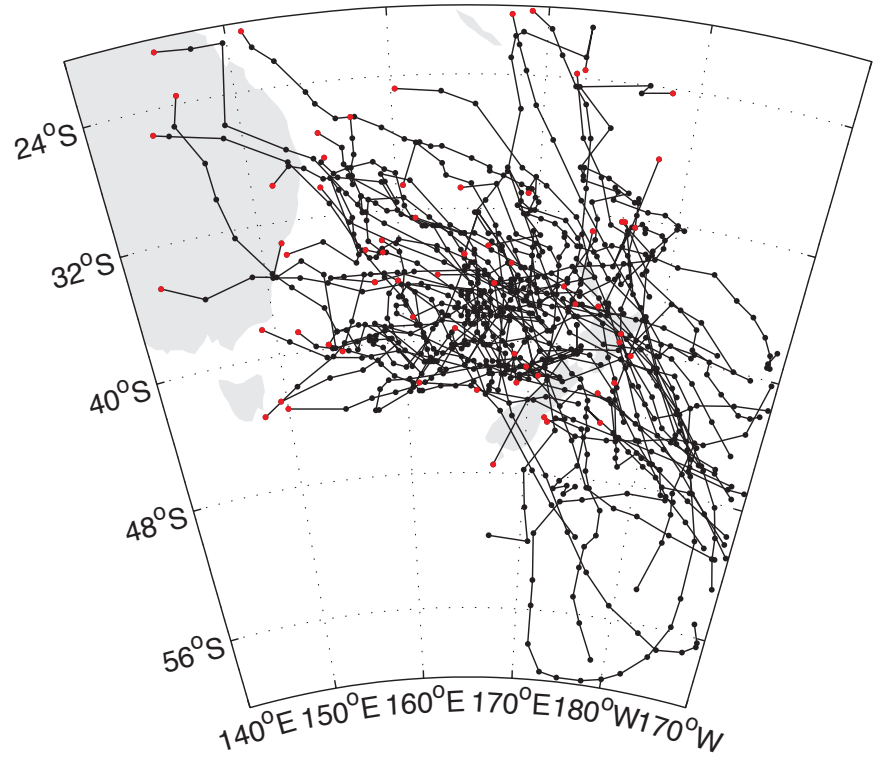

Figure $\quad 4.14 \quad$ Individual storm tracks that contained a geopotential height minimum within the New Zealand region on a day that coincided with heavy precipitation in the Waikato River catchment.

is consistent with observations in Sinclair (1995a) and with Sutcliffe's steering effect (Carlson, 1998) in the mid-latitudes. Another sub-group of tracks travelled in a more southerly direction from directly north of New Zealand.

Our tracking method missed Southern Ocean cyclones that can drag long cold fronts over New Zealand as their centres never passed close enough to the Waikato to be included in the list of tracks. Due to the breakdown of pressure tracking methods 
at these latitudes and the boundary of our selected region, these cyclones may not have been tracked anyway. To properly account for these storms a larger area and a different tracking method would be required. The method applied here focuses on cyclones originating near Australia, which have been observed in more than $50 \%$ of events. Cyclones originating in the Southern Ocean accounted for $16 \%$ of cyclones identified in Table 4.2, and should therefore account for a similar proportion of the cyclones discussed in this section. These cyclones will be among the 38 precipitation peaks that were not matched to tracked cyclones.

The frequency of a tracked-mean sea-level pressure minima passing through each geographical degree square were summed to distinguish between tracks where the centres travelled across different parts of New Zealand. Figure 4.15 shows (a) the frequency of minima in each matched track, and (b) the proportion of minima in each square that were associated with a storm track. The frequency of minima is greater in the

a)

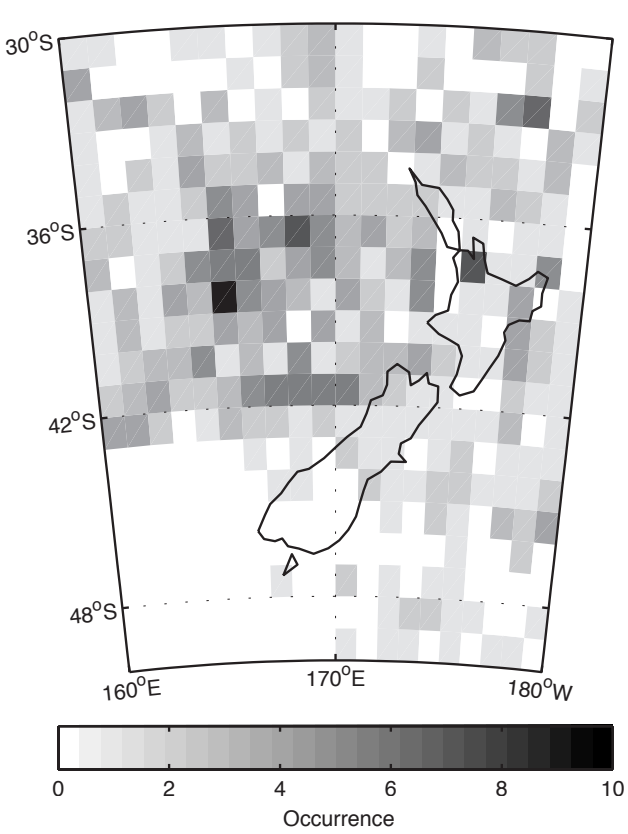

b)

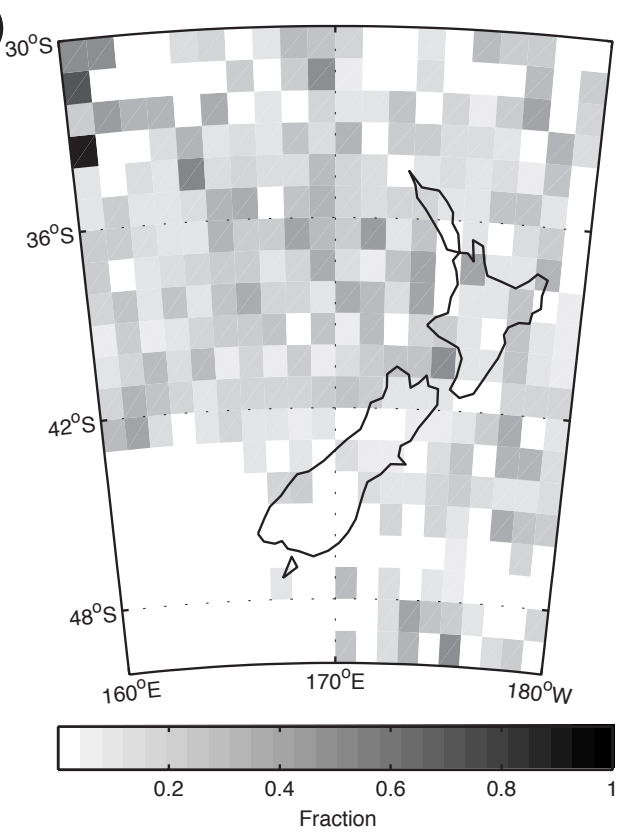

Figure 4.15 The frequency of geopotential height minima at each grid square during all tracks that (a) were identified as part of an event (shown in Figure 4.14) or (b) were identified as tracking through the New Zealand region during their lifetime.

Tasman Sea to the west of the North Island of New Zealand, both absolutely and proportionally. The high fraction of minima in the centre of the Tasman Sea could 
indicate that cyclones that stall here or pass through here are more likely to produce heavy precipitation. This is consistent with observations and is likely to occur when there is a blocking high.

The storm tracking method has shown that geopotential height minima can be tracked across the Tasman Sea in a predominately southeasterly direction. Only 10\% of cyclones will result in heavy precipitation in the Waikato River catchment, and those that linger in the Tasman Sea are more likely to cause heavy precipitation. The event analysis showed that cyclones crossing through these areas are responsible for the majority of heavy precipitation events. This tracking method has shown that only a small proportion of the cyclones that have developed in this area actually led to heavy precipitation and that the development of a cyclone is insufficient by itself to forecast heavy precipitation.

\subsection{Discussion}

This chapter analysed the characteristics of synoptic weather systems that have produced heavy precipitation in the Waikato River catchment, especially those that led to flooding. Geopotential height minima were then tracked through the Tasman Sea to determine the proportion of mid-latitude cyclones that were associated with heavy precipitation.

On average, five heavy precipitation events occur a year, producing an average of $44.8 \mathrm{~mm}$ of precipitation over the entire catchment. On average, an extreme in this catchment of $14,258 \mathrm{~km}^{2}$ equates to $0.64 \mathrm{~km}^{3}$ of water precipitating into the catchment during the event. The largest volume event during this period (according to the definitions applied here) resulted in $2.28 \mathrm{~km}^{3}$ of water being precipitated.

It was the high intensity events in summer, and long duration events in winter, that caused the largest volumes of precipitation in the Waikato River catchment. In many intense events the passage of a cyclone was stalled by the presence of a blocking high east of New Zealand, which can remain stationary for up to a week. This situation 
is caused by a pronounced meridional flow in the upper levels*. In contrast, the long duration events are often associated with a period of enhanced baroclinicity. During enhanced baroclinicity, cyclogenesis is more likely to occur resulting in a rapid sequence of cyclones passing over the New Zealand area.

Heavy precipitation is typically associated with the passage of a frontal band over the Waikato River catchment and these fronts are normally associated with midlatitude and subtropical cyclones (and occasionally an ex-tropical cyclone). The direction of the approaching front and the associated winds determine which part of the catchment is most likely to experience precipitation due to the enhancement and sheltering effects of the topography (discussed more in Chapter 6). However, the flooding potential is often dependent on the saturation levels of the ground. This is why cyclone sequences are associated with most major floods as the first features saturate the catchment and precipitation from later features lead to flooding.

Cyclones most frequently develop in the baroclinic troughs near the east coast of Australia (see Sinclair (1995a), (1995b), (1994), and Trenberth (1991) for details). These cyclones accounted for 52 of the 99 synoptic features identified in 63 events. In most cases a closed minimum was also observed in the $500 \mathrm{mb}$ geopotential height field. Tracking the $1000 \mathrm{mb}$ geopotential height minima used to classify these features as cyclones showed that only $10 \%$ of the cyclones developing in these conditions lead to heavy precipitation in the Waikato River catchment.

*Glossary of Meteorology, http://amsglossary.allenpress.com/glossary 
CHAPTER 5

\section{GLOBAL FORECASTS OF REGIONAL PRECIPITATION}

This chapter determines the accuracy and stability of the Global Forecasting System's (GFS) model precipitation forecasts to determine their value as an extended range forecast for the Waikato River catchment. These forecasts are also used to initialise and constrain the boundary of the mesoscale model used in Chapters 6 and 7 to simulate extreme precipitation events and climate respectively.

The accuracy of the GFS model is often evaluated over a large area, such as the tropics in Seo et al. (2005) or North America in Hamill et al. (2008) and Friederichs and Hense (2008). Other validation methods, such as cluster analysis (Marzban and Sandgathe, 2006) or contiguous rain areas (Ebert and McBride, 2000) are good for validating individual precipitation features. Unfortunately, both of these techniques also require a large number of grid points, and the Waikato River catchment only contains a single grid point (see Figure 5.1).

The GFS grid point precipitation forecast data have been analysed for accuracy using a number of common forecast verification parameters (Mailier et al. (2006) and Wilks (1995)). The basic parameters can be either categorical or time-series verification, and both have been applied in this study. Ensemble techniques are also commonly used to determine and improve the accuracy of forecasts (Atger, 2001; McBride and Ebert, 2000; Hamill, 1999; Du et al., 1997; Leslie and Holland, 1991) and have been trialled here.

Global models have been shown to be better at predicting the presence of precip- 
itation in an area than they are at predicting the magnitude or exact location of the precipitation (McBride and Ebert, 2000). The largest errors in the global precipitation forecast in New Zealand result from the model's failure to simulate orographic enhancement because of insufficient resolution of topography. Sinclair (1993) showed that the topographic resolution would have needed to be at least $10 \mathrm{~km}$ in order to simulate the magnitude of orographically enhanced precipitation that occurred during Cyclone Bola (a devastating ex-tropical cyclone that struck the North Island in 1988). It is still too computationally expensive to run global operational models at this resolution with today's technology.

The statistical accuracy of a precipitation forecast does not always reflect the value of the forecast (Mailier et al. (2006), Murphy (1993), Murphy and Winkler (1987), and Brier (1950)). Modellers often use statistical accuracy as it is objective and provides consistent accuracy measures between models. However, 'the best forecast according to the accepted system of arbitrary scores may not be the most useful forecast' (Brier, 1950). This is why later sections of this chapter attempt to determine the value of these forecasts using ensemble and probability of precipitation techniques.

\subsection{Data}

The operational numerical weather prediction model run at the National Center for Environmental Prediction (NCEP) provides the GFS data. This model output is freely available on the internet and a new model run is provided every six hours*. Although other global models do exist, since the inclusion of satellite data in 1979, there is good agreement between the daily geopotential height fields (Renwick, 2004), and thus between forecasts, from the main providers. The GFS model is more reliable but less skilful than the other major global model used for operational forecasts worldwide (Hamill et al., 2008), the ECWMF model. As the output of this model is being used to initialise further modelling, reliability is more important than statistical skill in this

${ }^{*}$ www.nco.ncep.noaa.gov/pmb/nwprod/analysis 
study.

GFS model output was available for a two-year period, August 2005-August 2007, for this study. Precipitation in this study is 'total precipitation $\mathrm{kg} / \mathrm{m}^{2}$ or code: APCP' in the model output. The model runs on a Gaussian grid but data are made available on a regular one-degree latitude/longitude grid (Figure 5.1). At each grid point the

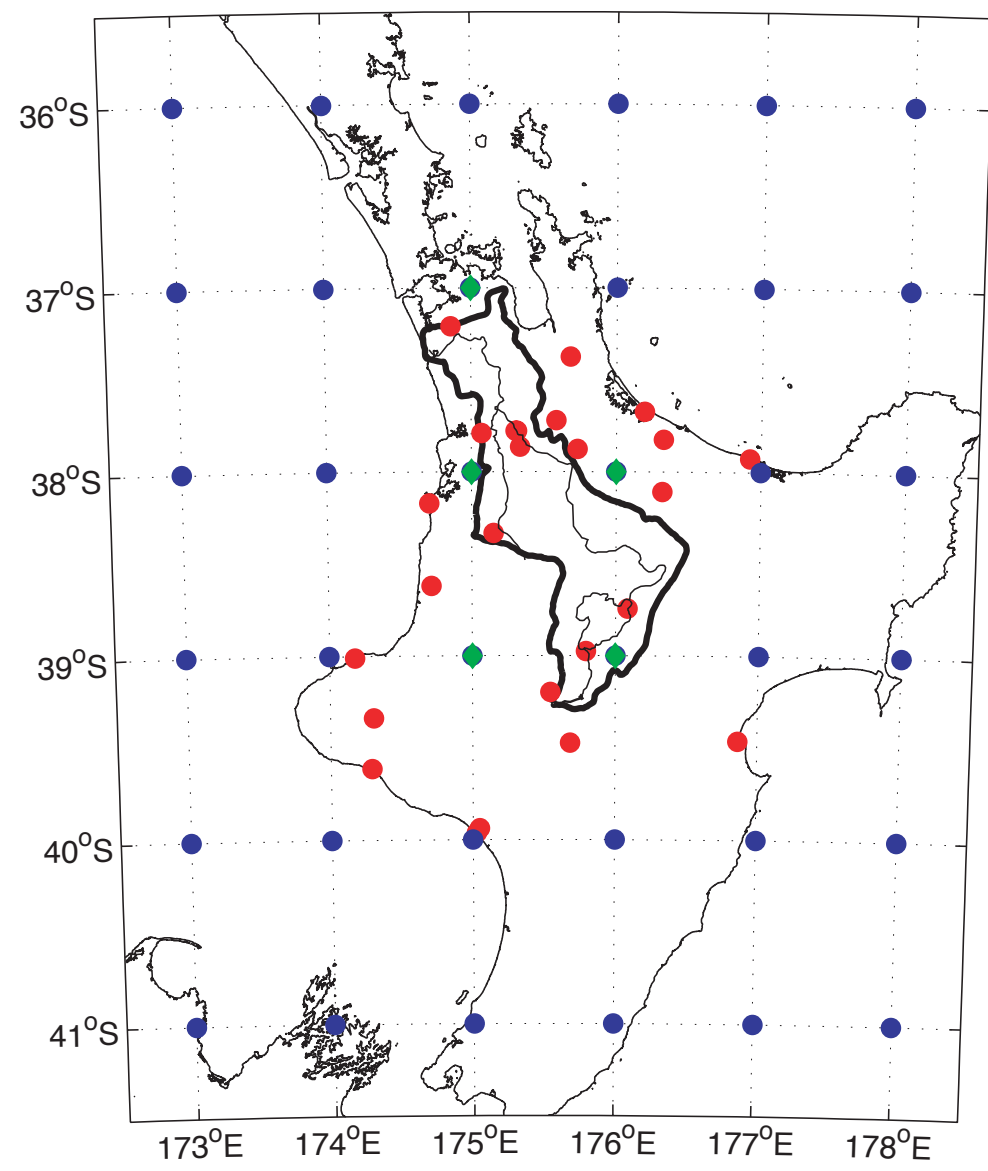

Figure 5.1 The location of hourly precipitation rain gauge stations (in red) in relation to the grid points where GFS data is provided (blue and green dots). The green dots represent the grid points included in area averages of grid point data.

accumulated precipitation at six-hourly intervals is provided out to 180 hours (this has since been extended to 384 hours, although at lower horizontal resolution).

The GFS model primarily uses spherical harmonic basis functions for spectral analysis, then the non-linear physics quantities and distributions are calculated. The topography used in the GFS model is that defined in Hong (1999). This model undergoes continual development, with the major components described in detail in Kanamitsu et al. (1991), Kalnay et al. (1990), and Kanamitsu (1989), and the current config- 
uration is described on a website ${ }^{\dagger}$. During the period of this study there were two upgrades, in August 2006 (Iredell, 2006) when infrastructural changes were made, and new orography and land/sea masks were included to improve forecasting in Antarctica and in glacial regions; and in May 2007 (Iredell, 2007) when data formats were adjusted to fit with other model output and a new vertical co-ordinate system (hybrid sigmapressure) was implemented. These changes were unlikely to have significant impact on precipitation predictions in the New Zealand region.

The current configuration calculates precipitation from the condensate in clouds, using both convective detrainment and grid scale condensation. Grid scale condensation is calculated according to Zhao and Carr (1997), which built on Sundqvist et al. (1989). With ice and evaporation parameterisations taken from Zhao and Carr (1997) and liquid water from Sundqvist et al. (1989), the model also allows for rain to evaporate in the unsaturated layers below the condensation level. All precipitation penetrating the bottom level is assumed by the model to fall to the surface.

In this study, the GFS data are validated against precipitation observations from the 23 rain gauge stations that recorded hourly observations in or near the Waikato River catchment. As discussed in section 2.2, models at this resolution cannot provide the spatial variation seen in observational data. Hourly observational precipitation data were extracted from the New Zealand Climate Database (CLIDB) at the locations shown in Figure 5.1. Precipitation observations were summed to provide corresponding six-hour accumulations at each station for the analysis.

Prior to a discussion of the statistical properties of the GFS data set, the six-hour $\operatorname{lag}^{\ddagger}$ forecast has been used to examine various properties of the available time series to determine the best fields to compare in the following statistical analysis of the GFS data. Data have been interpolated in this study to allow comparisons between the observations and forecast values. All interpolations in this study were carried out using a

\footnotetext{
${ }^{\dagger}$ http://www.emc.ncep.noaa.gov/gmb/moorthi/gam.html

${ }^{\ddagger}$ See Appendix $\mathrm{B}$ for definitions. This thesis follows the convention of referring to these forecasts by a lag time although in other studies they could be referred to as lead time or simply forecast.
} 
biharmonic spline interpolation scheme (Sandwell, 1987). Although interpolation is not ideal, without it we cannot compare the global forecast output to observational data.

The six-hour lag forecast is generally considered the most accurate as errors have had less time to propagate. Precipitation, particularly convective precipitation, is generated as the model progresses (Kanamitsu, 1989, model spin up) and will be underpredicted in the six-hour lag. Despite this, as the six-hour forecast is more constrained by the initial conditions it is used in this section to discuss general data attributes. The appropriateness of interpolation of this data for comparison is discussed below.

\section{Interpolation to grid or stations}

Observations are point values at their location, so interpolating grid data to their location means that only the estimated field is altered. However, the forecast provides a smoothed precipitation field over the larger area, and smoothing the observations in a manner similar to data assimilation may provide more comparable fields as suggested in Du et al. (1997). Both methods have been tested using the six-hour lag forecast and the difference between the time series plotted in Figure 5.2. All time series were averaged over the area prior to comparison.

For verification, NCEP averages all gauges within the grid square and the four surrounding model points and then compares those. Cherubini et al. (2002) used very high-density precipitation observations within each grid box to verify the ability of the ECMWF model. Verification against irregular and scattered observations is highly influenced by the variability of the precipitation in a grid box, e.g. Milbrant and Yau (2001) recorded a daily precipitation difference of $14 \mathrm{~mm}$ at two gauges less than $1 \mathrm{~km}$ apart. This means that a single grid point can be representing multiple precipitation microclimates.

When the forecast grid data are interpolated to the observation locations, there is a higher probability of over-predicting the precipitation (60\% of errors were negative) but the probability that the error will exceed $5 \mathrm{~mm}$ in any six-hour period is $20 \%$ less 


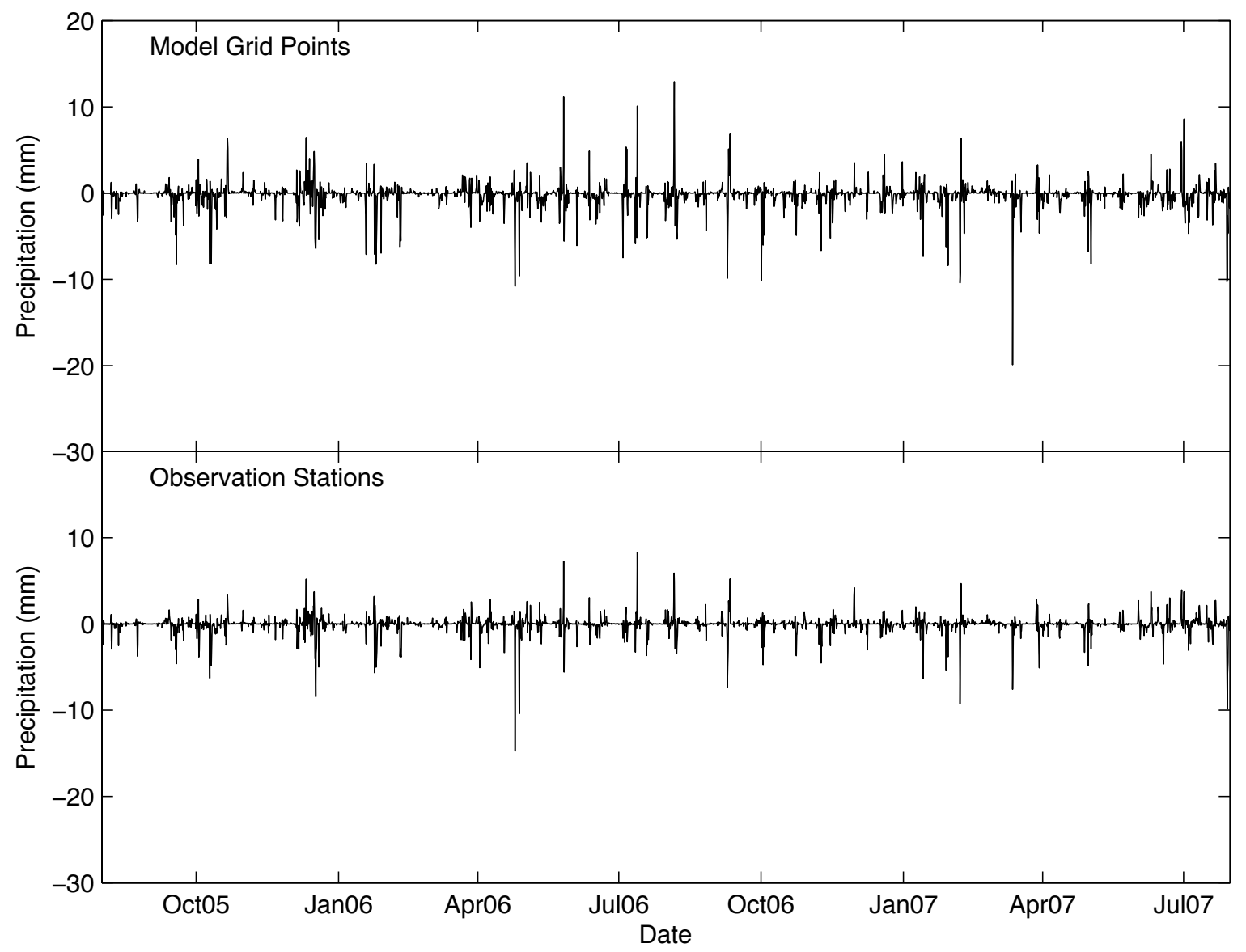

Figure 5.2 Time series difference after interpolation of data sets for comparison. The top plot shows the difference between the observation (average six-hour precipitation accumulations) and GFS (six-hour lag forecast data) time series when the observational data interpolated to the GFS grid points. The bottom shows GFS grid point data interpolated to observational stations. Positive values indicate that more precipitation was predicted than observed.

than if the observations were interpolated to the grid points. In this region, many of the surrounding grid points are in the sea and when the observations were interpolated to the geographical grid of the GFS model they were unconstrained on the ocean side, and this could be the cause of the larger errors. For this reason, the forecast data will be interpolated to the observation stations for the remainder of this chapter.

\section{Catchment wide or single station comparison}

In previous chapters the volume of precipitation was approximated by an area average.

This allows for a single value to represent the catchment. Averaging over the region 
will also reduce the effect of slightly mis-located precipitation that is still within the catchment and could reduce errors from the interpolation of forecast data to rain gauge stations. The individual errors for each station are plotted in Figure 5.3 along with the area-averaged error.

The area-averaged errors are much smaller than the errors for individual rain gauge locations (as expected), reaching a maximum error of $-14.7 \mathrm{~mm}$ in six hours instead of the maximum error of $-99.9 \mathrm{~mm}$ in six hours. The majority of all errors are positive and indicate that the tendency to over-predict precipitation occurs throughout the region. The large individual errors and small averaged errors could indicate that the amount of precipitation reaching the area is generally well predicted while the distribution of the precipitation is less accurate, which is as expected in a model that has limited spatial and topographical resolution.

\subsection{Categorical accuracy}

Categorical accuracy determines how often a forecast will predict a value within the same category as the observation. In many cases this is simplified to a yes/no question such as 'did it rain?'. Using this example, for both the observation and the forecast we can complete a contingency table (as in Table B.1 or 5.3) and use this to calculate multiple measures of accuracy, such as the hit rate, bias, probability of detection, false alarm rate and threat score (see Appendix B.4 for definitions of italicised terms). In some studies and large operational institutions such as ECWMF the hit rate is referred to as the 'percentage correct' and they use the term 'hit rate' synonymously with the 'probability of detection', here we follow the convention of Wilks (1995).

This idea can be extended to multiple precipitation categories as we have done in this study. Precipitation has been classified in the categories shown in Table 5.1. Beside each category, the proportion of six-hour periods observed in each category is provided to show relative occurrence rates. A full contingency table was then established for each forecast lag. The six-hour lag is shown here as an example in Table 5.2. 

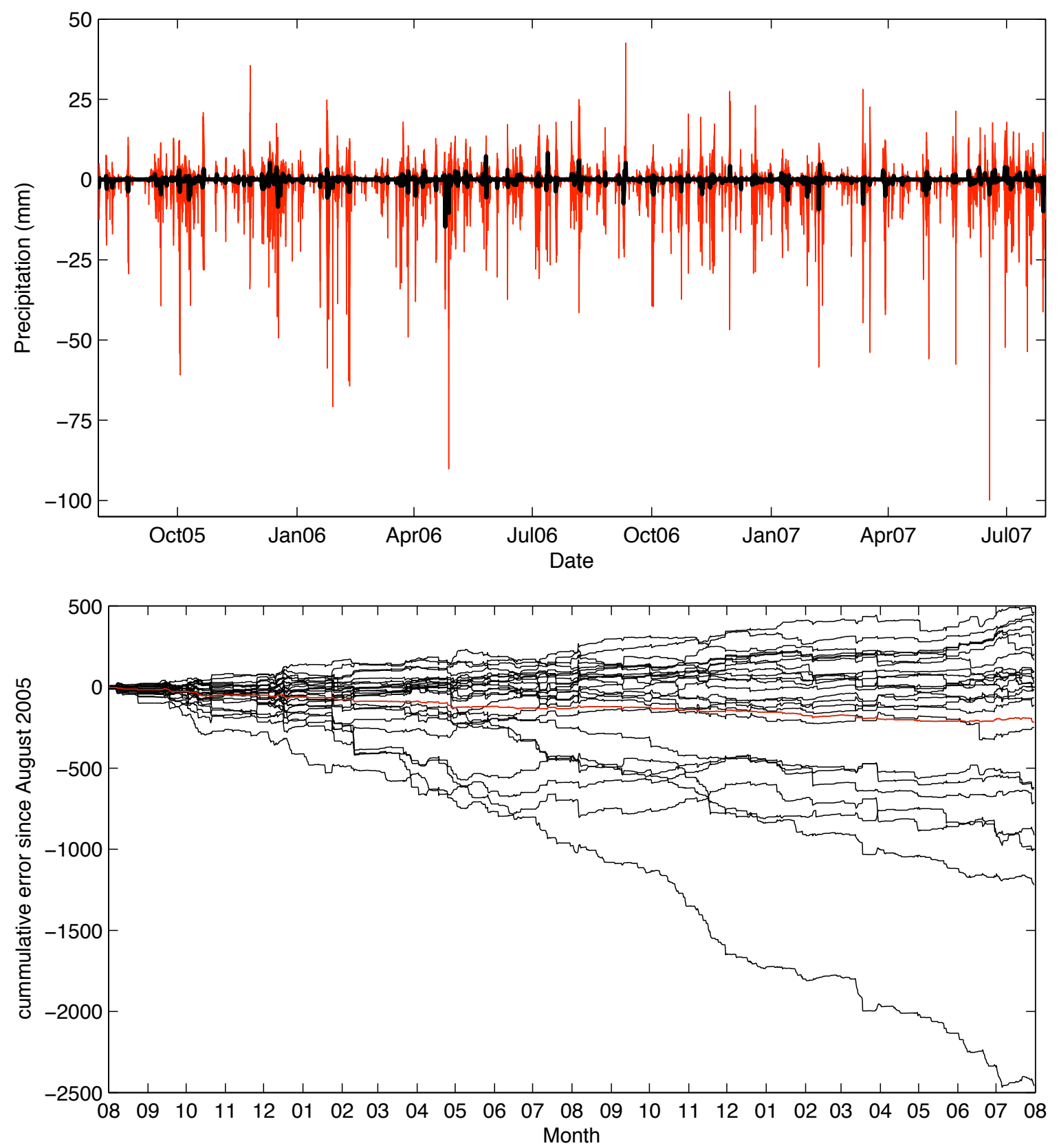

Figure 5.3 Comparison of time series errors between precipitation observation at each rain gauge and GFS data interpolated to the same location. The red lines (one per station) show the difference for each station compared to the interpolated prediction value for that point. The thickened black line shows the difference between the area-averages and is the same as the bottom plot in Figure 5.2. 
Table 5.1

Six-hourly precipitation categories used in this chapter and their natural occurrence probabilities

\begin{tabular}{|r|c|}
\hline Category & Occurrence \\
\hline$<1 \mathrm{~mm}$ & 0.845 \\
$1-5 \mathrm{~mm}$ & 0.092 \\
$5-10 \mathrm{~mm}$ & 0.031 \\
$10-15 \mathrm{~mm}$ & 0.011 \\
$15-20 \mathrm{~mm}$ & 0.005 \\
$>20 \mathrm{~mm}$ & 0.006 \\
\hline
\end{tabular}

Table 5.2

An example of the full categorical contingency table for forecasts with a six-hour lag. Observation categories are along the top and forecasts down the side.

Observations

\begin{tabular}{|c|c|c|c|c|c|c|c|c|}
\hline & $<1$ & $1-5$ & $5-10$ & $10-15$ & $15-20$ & $>20$ & Total \\
\hline \multirow{7}{*}{ Forecast } & $<1 \mathrm{~mm}$ & 54604 & 3523 & 740 & 214 & 71 & 65 & 59217 \\
\hline & $1-5 \mathrm{~mm}$ & 6207 & 3095 & 955 & 317 & 140 & 146 & 10860 \\
\hline & $5-10 \mathrm{~mm}$ & 739 & 599 & 451 & 184 & 86 & 88 & 2147 \\
\hline & $10-15 \mathrm{~mm}$ & 136 & 129 & 122 & 66 & 44 & 44 & 541 \\
\hline & $15-20 \mathrm{~mm}$ & 22 & 30 & 44 & 18 & 14 & 34 & 162 \\
\hline & $>20 \mathrm{~mm}$ & 20 & 16 & 19 & 13 & 15 & 15 & 98 \\
\hline & Total & 61728 & 7392 & 2331 & 812 & 370 & 392 & 73025 \\
\hline
\end{tabular}

There are two measures that can be calculated from a full contingency table: the categorical hit rate and the categorical bias. Both of these measures are shown as a function of lag in Figure 5.4. The categorical hit rate decreases with increased lag from 0.795 at the six-hour lag to 0.725 at the 180 -hour lag (i.e. the six-hour lag forecast matched 6,000 more observations than the 180-hour lag forecast).

The bias shows very little variation with lag. When more than $1 \mathrm{~mm}$ of precipitation is predicted, there is an increase in the bias between the six and 12-hour lags. This could relate to the spin up of the model and the lack of convective precipitation in the first hours of the model run. The $<1 \mathrm{~mm}$ threshold has almost no bias which is not surprising given the previous research (McBride and Ebert, 2000) identified that the model could identify dry periods. Light precipitation $(1-5 \mathrm{~mm})$ is predicted $1.5-1.8$ 

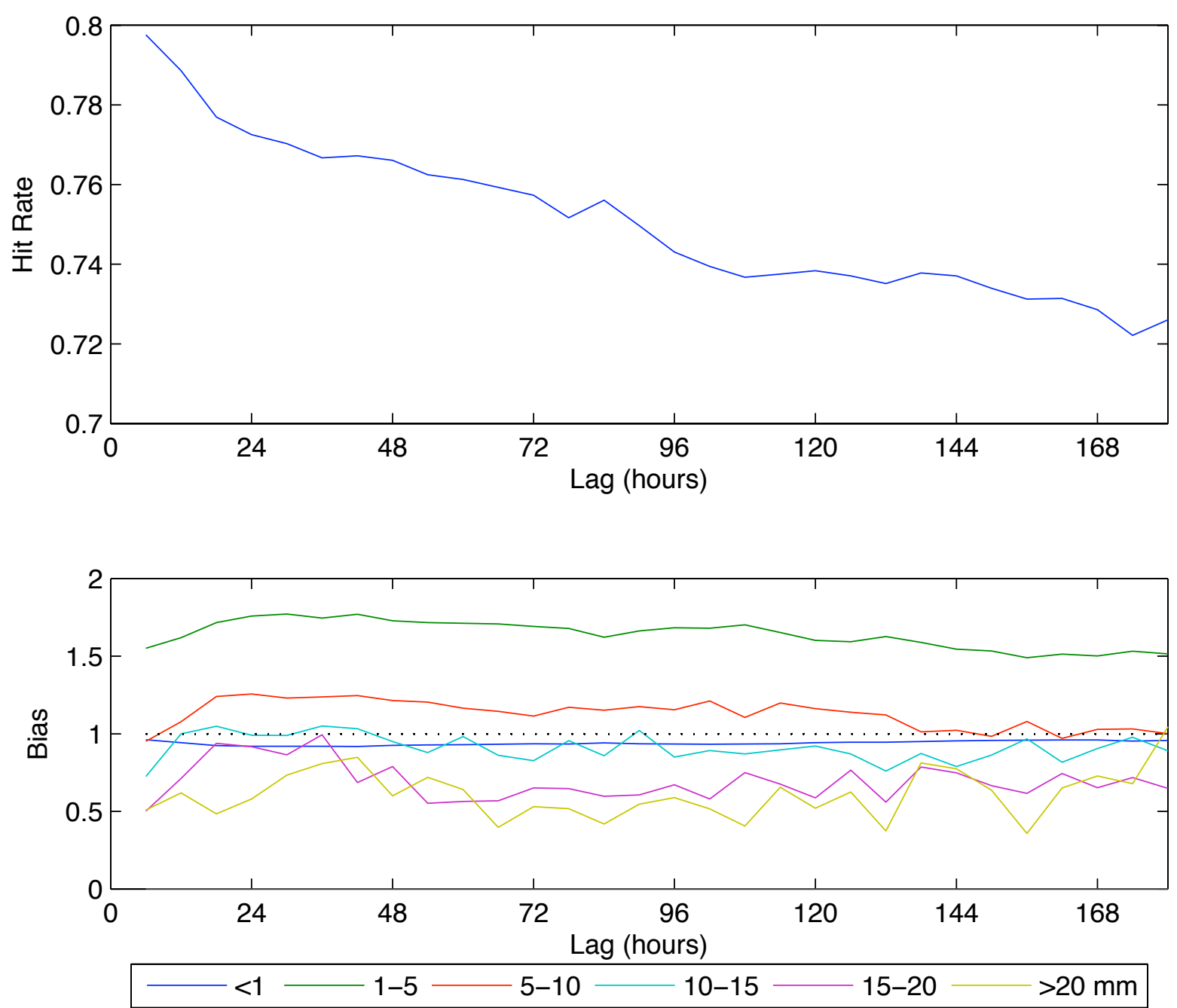

Figure 5.4 The categorical hit rate (top) and bias (bottom) as functions of lag.

times more frequently than it occurs. However, for heavy precipitation $(>15 \mathrm{~mm})$ the bias can be as low as 0.5 although the occurrence and therefore statistical significance is low.

For the other measures mentioned at the start of this section the contingency table has to be collapsed to a binary system as shown in Table B.1. To maintain the categories the contingency table was split into five tables using the category boundaries as thresholds, e.g. 'was there greater than $20 \mathrm{~mm}$ of precipitation', which has the contingency table shown in Table 5.3 at a six-hour lag. Then for each threshold the hit rate, bias, probability of detection, false alarm rate, and threat score were calculated as functions of lag and plotted in Figure 5.5. 

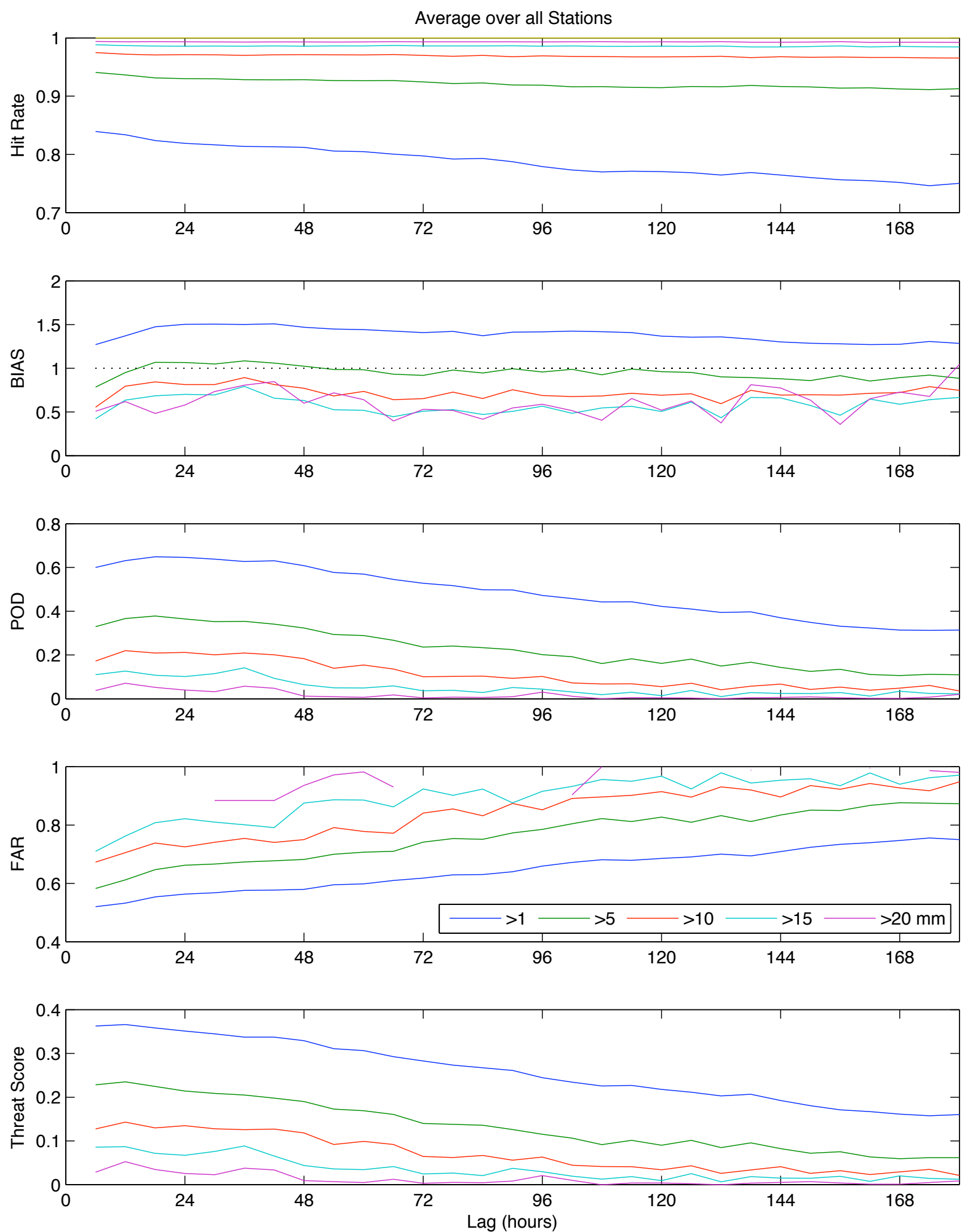

Figure 5.5 Categorical error analysis as a function of lag (hours). The five categorical accuracy measures (hit rate, bias, probability of detection, false alarm rate, and threat score) were calculated for each individual station at each lag separately for a series of precipitation exceedance thresholds. The plots show the average value across all stations for each forecast time at each threshold. 
Table 5.3

An example of the categorical contingency table for forecasts with a six-hour lag predicting greater than $20 \mathrm{~mm}$. Observation categories are along the top and forecasts down the side.

\begin{tabular}{cc|c|c||c} 
& \multicolumn{4}{c}{ Observed } \\
& & yes & no & Total \\
\cline { 2 - 5 } Forecast & Yes & $\mathbf{1 5}$ & 83 & 98 \\
\cline { 2 - 5 } & No & 377 & $\mathbf{7 2 5 4 0}$ & 72927 \\
\cline { 2 - 5 } & Total & 392 & 72633 & $\mathbf{7 3 0 2 5}$
\end{tabular}

The hit rate shows that the higher the precipitation threshold, the more accurate the forecast. This is misleading, however, as the contingency table becomes dominated by the 'was not forecast and did not happen' cell of the table, a well known consequence of this measure for rare events such as heavy precipitation, fog or tornados etc. (Wilks, 1995). The hit rate decreases with lag in each category. Further, the hit rate of the $>1$ $\mathrm{mm}$ of precipitation category threshold mirrors that of the categorical hit rate in Figure 5.4, suggesting that the accuracy of the forecast, using this measure, is dominated by the model's ability to predict dry weather.

The bias calculations show almost no bias (bias=1) at the $>5 \mathrm{~mm}$ threshold. At the lower threshold their bias is positive, while at higher thresholds the bias is negative. Therefore, when the model predicts precipitation, it preferentially predicts lighter precipitation. For all thresholds there is an increase in bias between the six and 12-hour lags, and the largest bias is in the 36-hour lag.

The highest probability of detection is close to the 24-hour lag for all thresholds, peaking at 0.65 for the $>1 \mathrm{~mm}$ category. However, for thresholds larger than $10 \mathrm{~mm}$, the probability of detection peaks below 0.2 and decreases as the threshold increases. The false alarm rate shows that the rate of predicting false alarms increases with increased lag, from $0.50-0.75$ for a threshold of $1 \mathrm{~mm}$ and from $0.70-0.95$ for a threshold of 10 $\mathrm{mm}$. This indicates that even if heavy precipitation did occur in the forecast it is unlikely to have been accurately predicted.

The threat score indicates the accuracy of the forecasts that matter, i.e. in this 
case those where precipitation occurs. The threat of precipitation being over the $1 \mathrm{~mm}$ threshold ranges between 0.37 and decreases to 0.16 over the lag range. The threat of precipitation being over $15 \mathrm{~mm}$ is never greater then 0.10 . This shows that there is a low threat of these events happening, but from the other measures we can also see that when they do happen they are not very well predicted, and longer time-lags are even less likely to be accurately predicted.

\subsection{Time series accuracy}

Categorical analysis can account for a 'hit or miss' scenario, but does not show any relationship between the actual values. Thus the observation time series were compared to forecast time series for each lag. When comparing two time series a number of measures can be used to determine how well they are correlated - the mean error, the root mean squared error (RMSE), the mean absolute percentage error (MAPE) the skill score, and cross correlation. These are all plotted as functions of lag in Figure 5.6, but they are also influenced by the abundance of dry periods.

The mean error is small $(<0.15 \mathrm{~mm})$ at all lags. However, there is a negative bias in the six-hour lag and a positive bias in the 18-hour lag, with the mean error approaching zero for longer lags. This indicates that precipitation is under-predicted during the spin-up of the model, and then this error is compensated for during the next few model steps. The root mean squared error shows an increase with lag from $2.8 \mathrm{~mm}$ to $3.8 \mathrm{~mm}$. The MAPE increases over the lags, at the shorter lags the MAPE is below $30 \%$ but at the longer lags it increased to nearly $60 \%$. This indicates that there is more likely to be an error in the percentage of the precipitation that can be predicted at longer lags.

Different forecast models are often verified in terms of a skill score. In this case it is comparing the skill of GFS model to the forecasting skill of assuming a climatological average (here that means assuming that $0.88 \mathrm{~mm}$ of precipitation fall in each six-hour period). The climatology is more skilful (smaller variance) at all lags greater than 18 

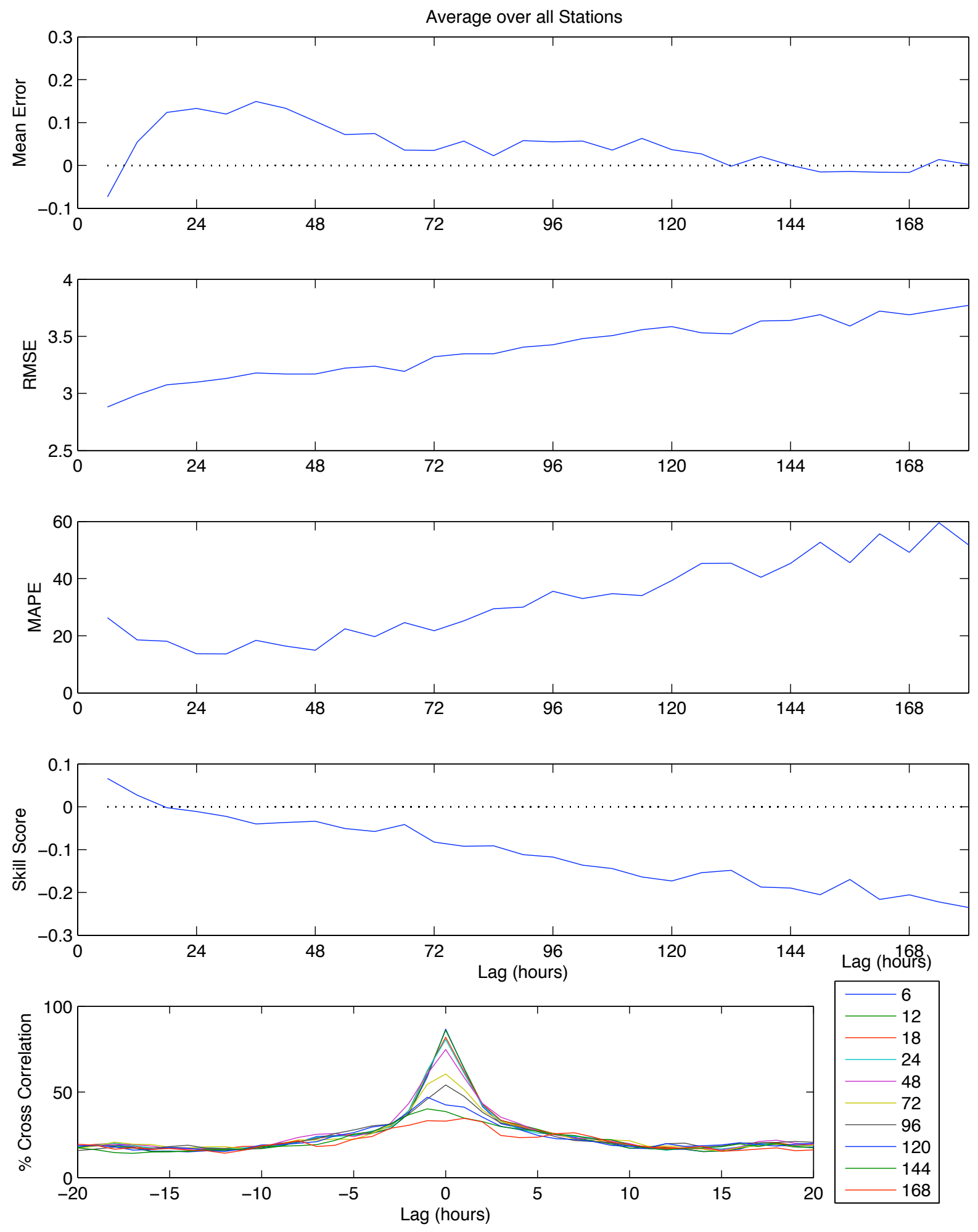

Figure 5.6 Continuous error analysis as a function of lag. The time series comparison accuracy measures (mean error, root mean square error, skill score, and the cross correlation) were calculated for each individual station at each lag separately. The plots show the average value across all stations for each forecast time 
hours and becomes even more skilful with increasing lag.

A cross-correlation was also calculated for each lag. At shorter lags a significant portion $(85 \%)$ of the time series are correlated. With longer lags or if one of the time series is shifted along the time axis, the correlation drops to $\approx 20 \%$.

These time series accuracy measures indicate that errors increase with lag. The negative bias at the six-hour lag is compensated for by a positive bias in the next model steps. However, in terms of short-term forecasting this means that there is an under-prediction of precipitation in the six-hour lag and over-prediction between the 12- and 130-hour lags.

The magnitude of the errors is again dominated by the uneven distribution of precipitation values. To determine the spread of errors as a function of lag, a box and whisker plot was created for each lag showing the range of forecast errors for days when precipitation was observed (Figure 5.7). This shows that the mean and interquartile ranges at all lags were approximately zero. Figure 5.7, showing the 1st and 99th percentiles, shows that large errors are evenly distributed between positive and negative, although the spread increases at longer lags as suggested in the previous analysis. The enlargement in 5.7 shows that for the six-hour lag and lags $>144$ hours, the distribution is approximately even about the mean at zero; at the intermediate lags there is more range in the positive errors (over-prediction) despite very similar medians.

\subsection{Error distribution}

Some of the errors in the model are likely due to low resolution. It is therefore of interest to show how some accuracy measures vary between observation stations. In this section the categorical hit rate and 'no precipitation' categorical bias, as well as the mean error and root mean squared error over all lags are mapped for individual rain gauge locations. Over 87,600 forecast/observation pairs are included in each calculation.

The categorical hit rate (Figure 5.8a) varies between 0.67 and 0.80 at different 


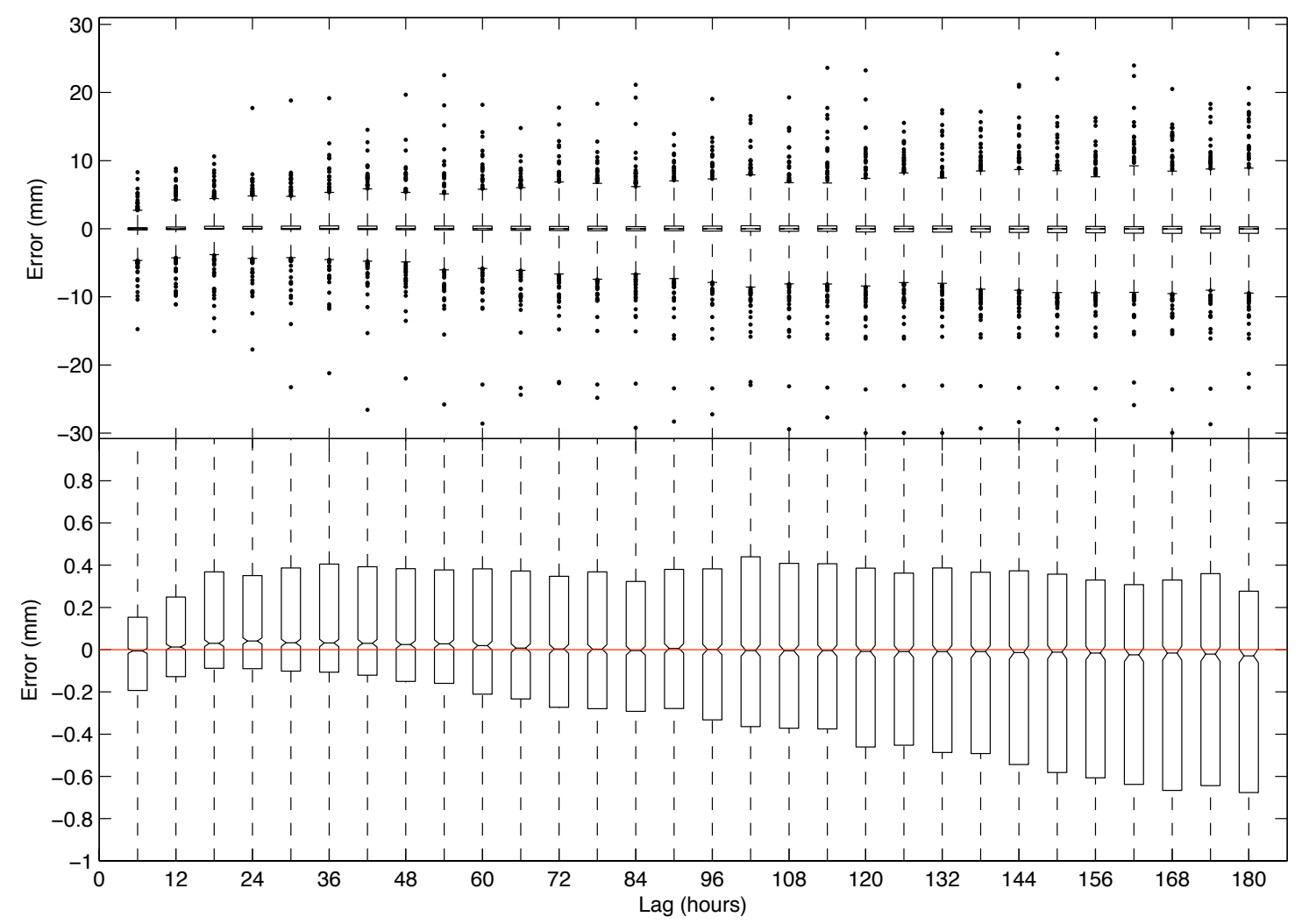

Figure 5.7 The distribution of forecast errors on days when precipitation occurred as a function of lag are shown through this box and whisker plot. The error between each observation and its corresponding forecast value are grouped for all stations and the range of errors are shown through a box and whisker for each lag. The box indicates the 25th, 50th and 75th percentiles and the whiskers extend to the 1st and 99th percentiles. The bottom plot is a vertical enlargement of the centre of the top plot to clearly show the medians and interquartile ranges.

stations. The stations located on the central plateau have the lowest hit rates. The categorical bias for the 'no rain' category (Figure 5.8b) ranges between 0.87 and 1.06. The only positive bias (over-prediction of dry days) is at Mt Ruapehu and smaller negative biases occur along the west coast. Further east, larger negative biases were calculated.

The RMS errors (Figure 5.8c) vary between 2.9 and $4.9 \mathrm{~mm}$. In general, this map shows that larger RMSE values were calculated for the stations that record exceptionally high precipitation totals during extreme events, such as Mt Egmont/Taranaki, and Tauranga. In comparison, stations in the centre of the Island, near the head of the 

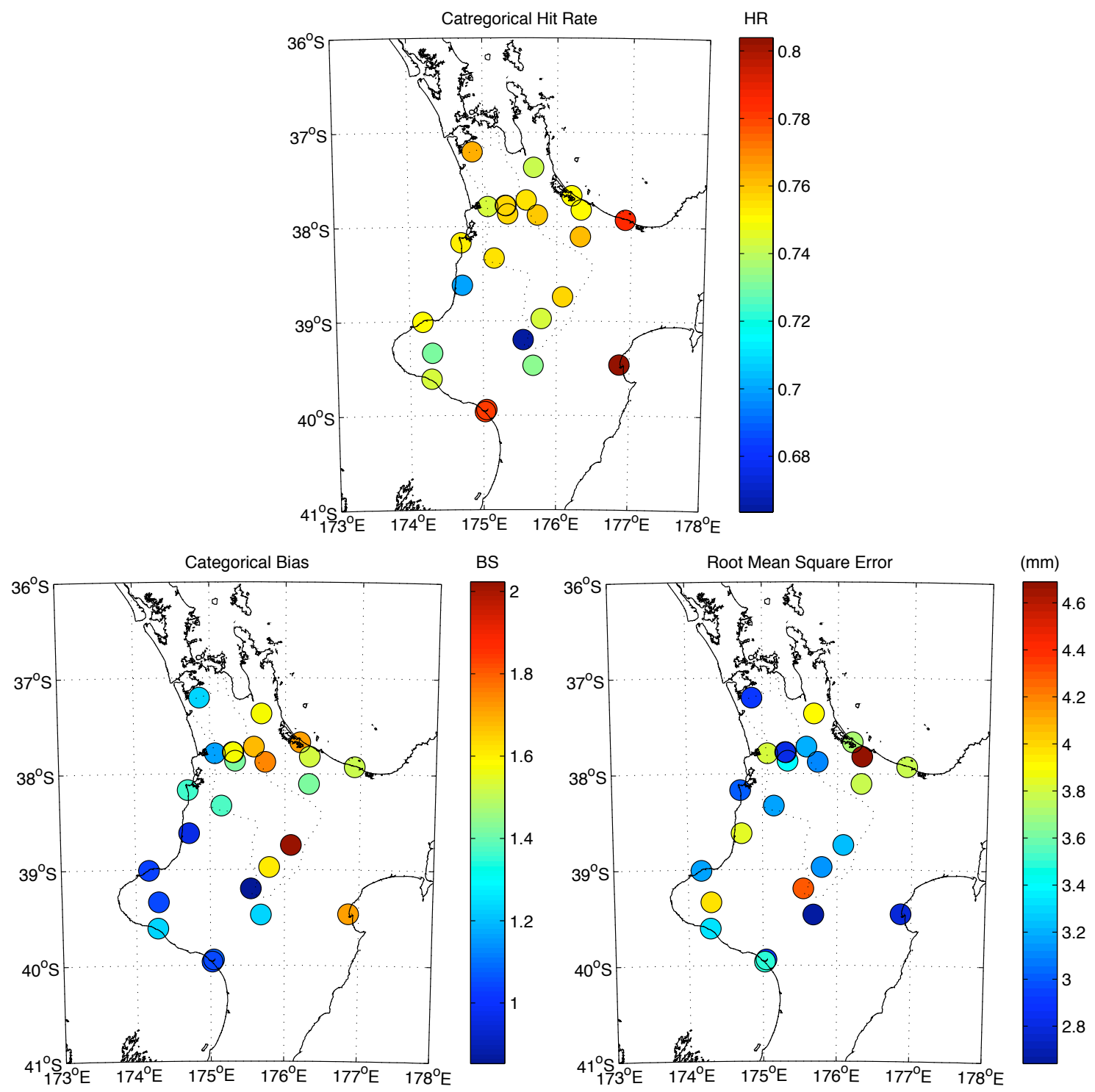

Figure 5.8 Statistical accuracy measures for individual stations over all lags: (a) the categorical hit rate, (b) the categorical bias for the $>1 \mathrm{~mm}$ rain category, (c) the average root mean square error over all lags.

Waikato River, with the exception of Mt Ruapehu, show smaller RMSE values.

The range in values for the mean error at different lag times is shown in Figure 5.9. Around the region the mean errors are small, between -0.8 and $0.3 \mathrm{~mm}$ with standard deviations between 2.6 and $4.6 \mathrm{~mm}$. In general there is a slight tendency to over-predict precipitation in the northwest and within the Waikato river catchment, and to slightly under-predict at stations in the southwest. The stations with the largest mean errors also had the largest standard deviations indicating that these errors had 

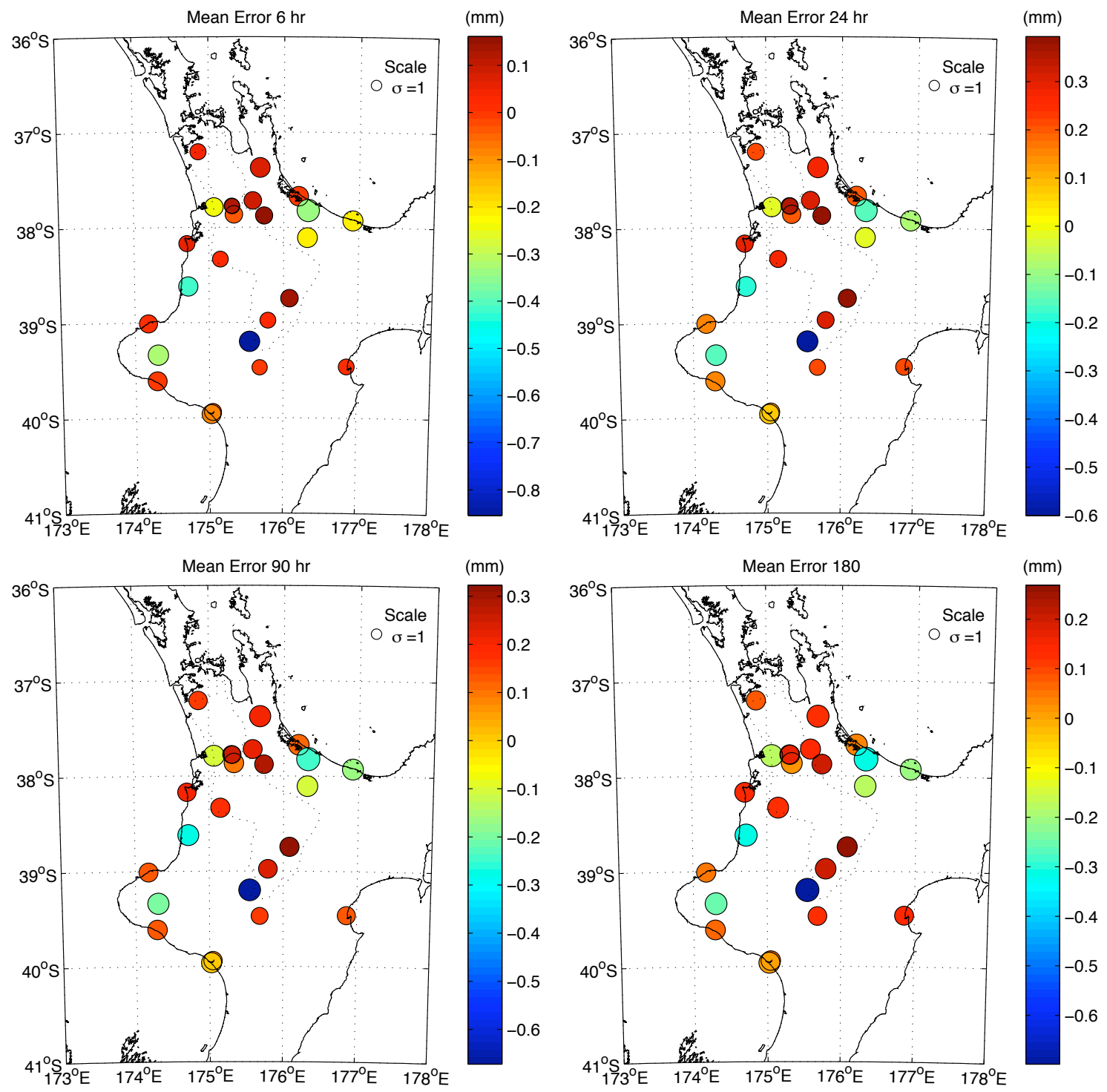

Figure 5.9 Statistical accuracy measures for individual stations over all lags: (a) the categorical hit rate, (b) the categorical bias for the 'no rain' category, (c) the mean error, and (d) the root mean square error.

more variability and the standard deviation also increased with increased lag.

The categorical measures show that stations around the coast tend to have higher hit rates, and inland stations show a more positive bias (over-predicting precipitation). The mean error shows that there is a tendency to over-predict the amount of precipitation in the northwest and within the Waikato River catchment, and the largest RMSE values are seen around the coastlines where larger extremes are observed, and at the Mt Ruapehu which is at higher altitude. 


\subsection{Ensemble forecasting}

Multiple model runs can be combined in ensemble forecasting to determine probabilistic forecasts of precipitation, and the consistency of the predicted meteorological features. These ensemble techniques have been shown to improve the skill of weather forecasting (Du et al., 1997; Leslie and Holland, 1991; Atger, 2001; Dalchér et al., 1988). As additional model runs could not be conducted, the model output were grouped here by the time for which the forecast was valid (valid time) to create an upto 30-member time lag ensemble. In this study all lagged forecasts are treated as being as likely but in reality the forecasts with shorter lags are more likely and the lags should be weighted accordingly.

Plotting the area average precipitation values from each model run as a function of lag (Figure 5.10, each line represents a single model run) shows the variation in predicted precipitation from approaching systems. Approximate periods of precipitation can be traced through the model runs. However, the magnitude of precipitation and exact timing varies slightly between runs.

As an example, the forecast pressure, wind and precipitation for a single valid time ensemble were plotted in Figure 5.11. The valid time here is 1800 UTC 24 January 2006, and coincides with the largest daily precipitation value observed during the twoyear period (more details of this event are presented in case studies in Chapters 4 and 6). The central minimum of this cyclone is located in a different position at different lags, located to the northwest in the 78-hour lag compared to the 144-hour lag where the pressure minimum is to the northeast. Further, at some of the long lags the synoptic situation is completely different with no pressure minimum visible at this valid time (e.g. 168- and 180-hour lags).

Events often appear to disperse and in $25 \%$ of the 207 precipitation periods during the two-year period, one of the model runs predicted continuing precipitation after the passing of the main precipitation event. In Figure 5.10 this can be seen in the model run initiated at 0600 UTC on 21 January 2006 (indicated by arrow in Figure 


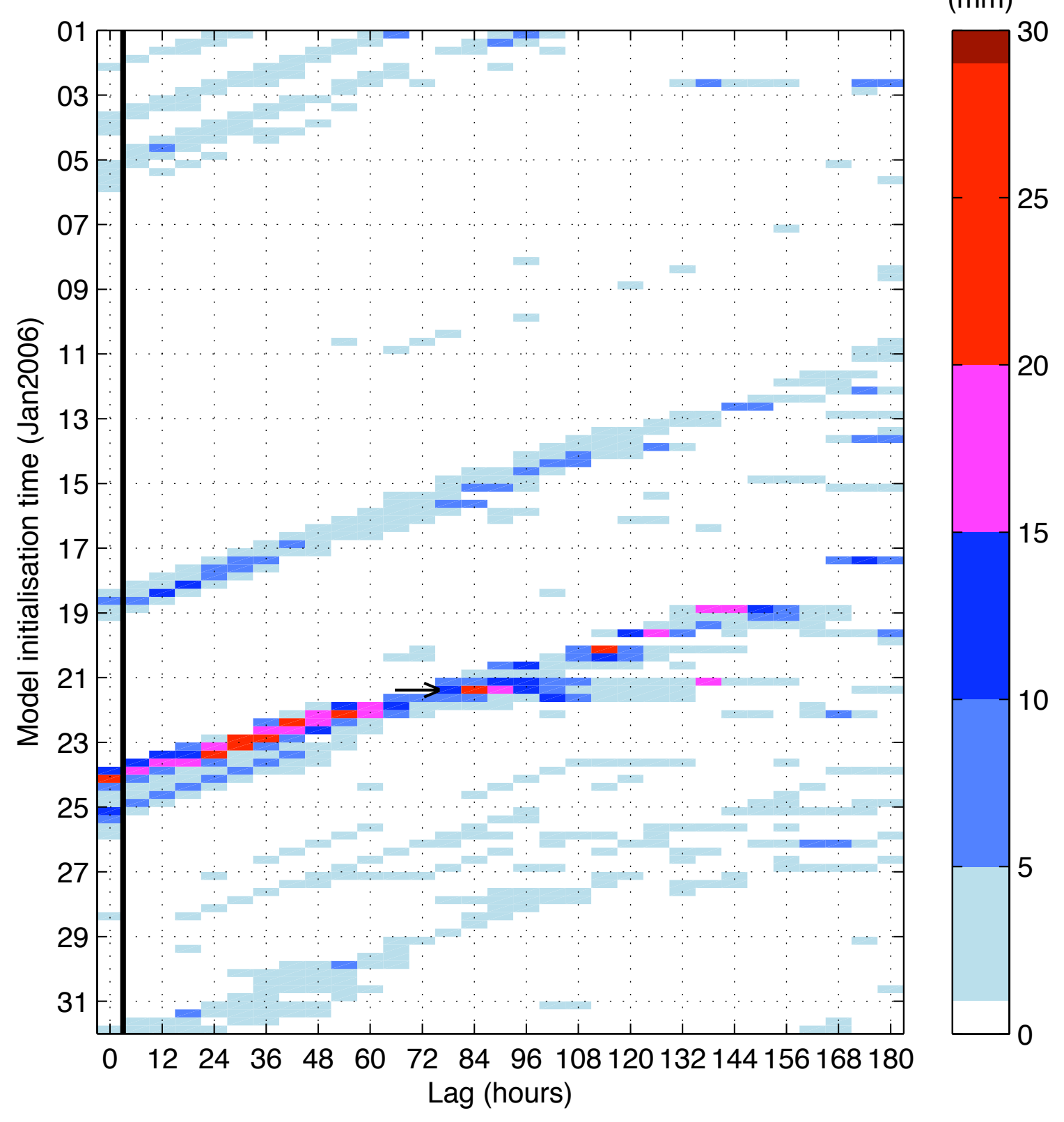

Figure 5.10 Complete precipitation runs issued for the month of January 2006. Each horizontal line in the plot is a single model run. On the left at ' 0 ' lag is the observed regionally averaged precipitation accumulation at the initialisation time of the model run. The arrow indicates the 0600 UTC run on 21 January 2006 that is discussed in the text.

5.10). This could be due to excess moisture in the atmosphere from lack of orographic enhancement, or the decay of the mesoscale feature. However, the forecast charts did not show a large variation between this model run and the runs initiated either six-hours before or after. 


\section{4-Jan-2006 1800 Ensemble}

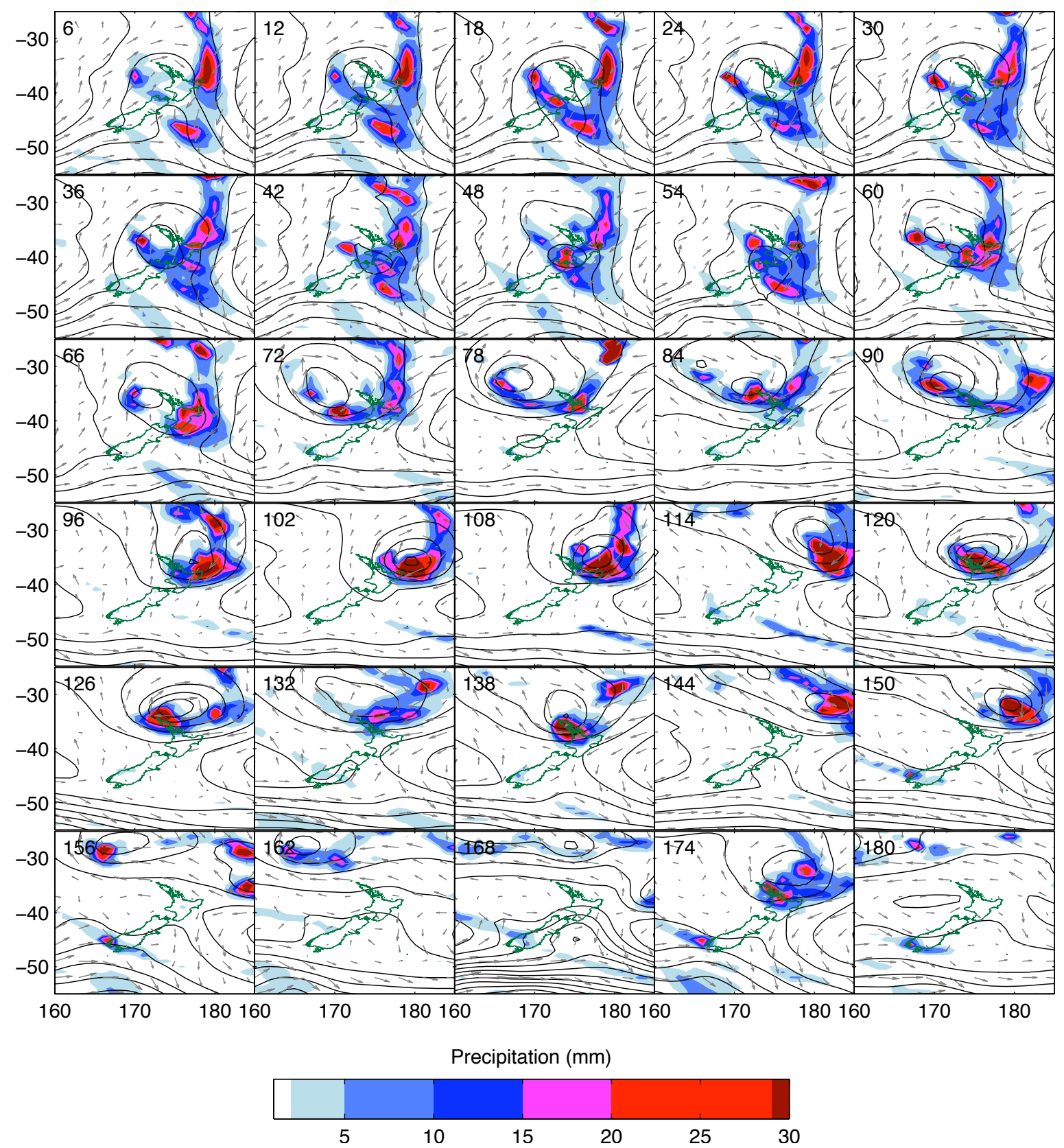

Figure 5.11 Each panel in this plot represents the forecast pressure, winds and precipitation for the valid time of 1800 UTC 24 January 2006 from each of the thirty model runs that included this valid time. The lag in hours is shown in the top left of the panel. 
The model predicts a precipitation event in most cases but the actual magnitude, duration and commencement time of the precipitation varies between model runs. Most of this error is likely a result of lower spatial resolution, poor representation of the topography and land/sea boundary and mean that the model will lack the orographic enhancement that is known to occur. As noted in Hamill et al. (2008), in addition to limited resolution of global models, errors also occur because of insufficient representation of clouds dynamical and microphysical processes. However, the failure of some model runs to identify the dominant mesoscale features indicates that there are small-scale variations in the lead up to the feature that may prevent or cause it to develop. The variations between model runs account for the poor accuracy especially at long lags where there is more time for errors to compound and alter the atmospheric development.

\subsubsection{Range of errors}

Large observed precipitation volumes appear to have a larger range in predicted values at the same valid time (Figure 5.12). However, a larger range (in the 30-member
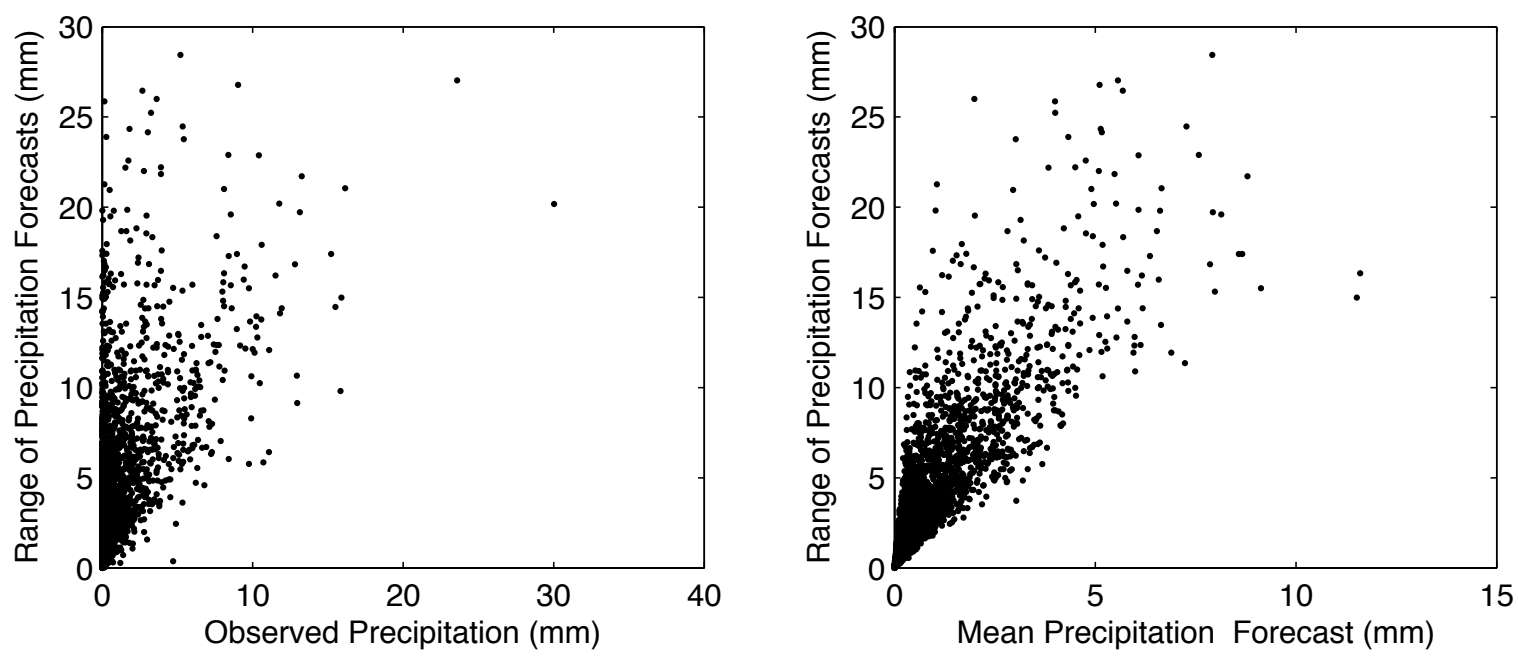

Figure 5.12 Mean and spread of precipitation forecasts at same valid time: (a) The observed precipitation value compared to the range of forecasts values for that particular valid time, (b) the mean precipitation value forecast for each valid time compared to the range of precipitation values forecast for that valid time. 
ensemble) in the predicted values does not always correspond to a larger observed value.

There is a correlation between the mean and the range of predicted values, the range is approximately three times the mean value. Regardless of the observed value, the minimum predicted value was zero or very near to it at every valid time. This also means that the range of values is very strongly correlated with the maximum value predicted.

The range of predicted precipitation values over the time-lag ensemble generally increased with increased observed precipitation. This relationship is weak and cannot be directly used to improve the forecast precipitation amount. However, it could be incorporated into a probability of precipitation calculation.

The area values were also simplified to a binary system to test the persistence of precipitation forecasts (Figure 5.13). The accuracy measures in earlier sections iden-
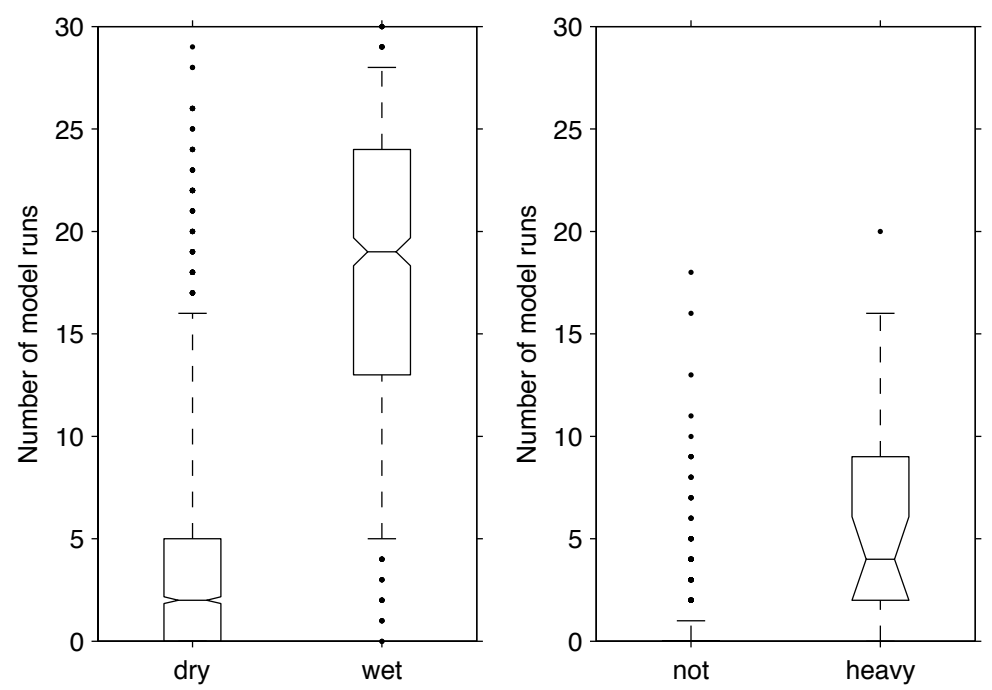

Figure 5.13 The spread of model runs correctly identifying precipitation or heavy precipitation. These box and whisker plots (1st, 25th, 50th, 75th and 99th percentiles) show the number of models that (a) predicted precipitation on observed 'wet' $(>1 \mathrm{~mm})$ and 'dry' $(<1$ $\mathrm{mm})$ days; and (b) predicted heavy $(>10 \mathrm{~mm})$ precipitation on days that observed heavy precipitation and those that did not.

tified that the model's accuracy was dominated by dry periods. Figure 5.13a shows the spread in model members that predicted precipitation during each observed wet and dry period. In both categories there are situations where no model run predicted 
precipitation and all model runs predicted precipitation. In $99 \%$ of periods where precipitation occurred, at least five, and a median of 19 model runs predicted precipitation. Likewise, less than five model runs predicted precipitation in $75 \%$ of dry periods, and fewer than 16 runs in $99 \%$ of dry periods. This shows that precipitation is more likely to be predicted on a day when it occurs. But there is considerable overlap in the number of models predicting precipitation in both scenarios.

Similarly the spread in the number of models that predicted heavy precipitation (with a threshold of $10 \mathrm{~mm}$ ) were compared in Figure 5.13b. This shows that in $99 \%$ of cases, heavy precipitation was not predicted when it did not occur. However, in two cases over the two years more than 15 model runs predicted a non-observed heavy precipitation period for a particular valid time. When heavy precipitation did occur the median number of model runs predicting heavy precipitation was only four runs. This also shows that heavy precipitation is unlikely to be predicted in many model runs even if it does occur.

\subsection{Probability of precipitation}

As model runs can provide inconsistent precipitation forecasts, it may be more valuable to the end user to be presented with a probability of the precipitation value being below specified values. These can then be combined so that the probably can be adjusted depending on the number of previous forecasts that predicted the same amount of precipitation (similar to that done in Gallus et al. (2007)). This is valuable as precipitation is more frequently forecast for periods where it occurs and these probabilities can reflect that. Statistical convention defines these distributions as below specified values, one minus the probability will give the probability of exceeding the specified value, which is more frequently used in meteorology. The 'probability of precipitation' concept was defined by Brier (1950), and the idea was developed using probability distribution functions in Murphy and Wilks (1998). As the data set contains more than 70,000 data points, the probability distribution function can be approximated by the 
cumulative frequency distribution.

Probability distribution functions can be used to determine the probability of receiving less than a particular precipitation value. This means that for any value of precipitation $\left(\mathrm{r}_{B}\right)$ in time series $\mathrm{B}$ (e.g. a time series of precipitation forecasts with the same lag), the probability of receiving less than $\mathrm{r}_{B} \mathrm{~mm}$ of precipitation can be determined as:

$$
F\left(r_{B}\right)=\int_{0}^{r_{B}} B=\int f\left(r_{B}\right) d r_{B}=\operatorname{Pr}\left\{B \leq r_{B}\right\}
$$

To compare two time series, the joint probability of both being below threshold values in both time series A (e.g. the subsequent forecast time series) and B can be calculated by:

$$
F(A, B)=\operatorname{Pr}\left\{A \leq r_{A} \bigcap B \leq r_{B}\right\}
$$

This can be used to determine the conditional probability of $A \leq r_{A}$ given $B \leq r_{B}$ for a range of $r_{B}$ values as in:

$$
F(A \mid B)=\frac{F(A, B)}{F\left(r_{B}\right)}
$$

The probability distribution function has been calculated for a series of $r_{B}$ thresholds to show how the probability varies over the range of precipitation values. An example using the 48-hour lag forecast time series as B is plotted in Figure 5.14a. The joint and conditional probabilities of the 48-hour lag series versus the 42-hour lag series are shown in Figure 5.14b and c for a range of predicted precipitation values. As there are a large portion of dry periods during our study period, the probability of observing less than $0.2 \mathrm{~mm}$ is greater than 0.65 regardless of the forecast. The joint probability shows that if one of the forecasts predicts $<0.2 \mathrm{~mm}$ of precipitation then there is an equal probability of receiving any precipitation value in the subsequent forecast. The conditional probability shows that the lower the prediction in the 48-hour forecast the 

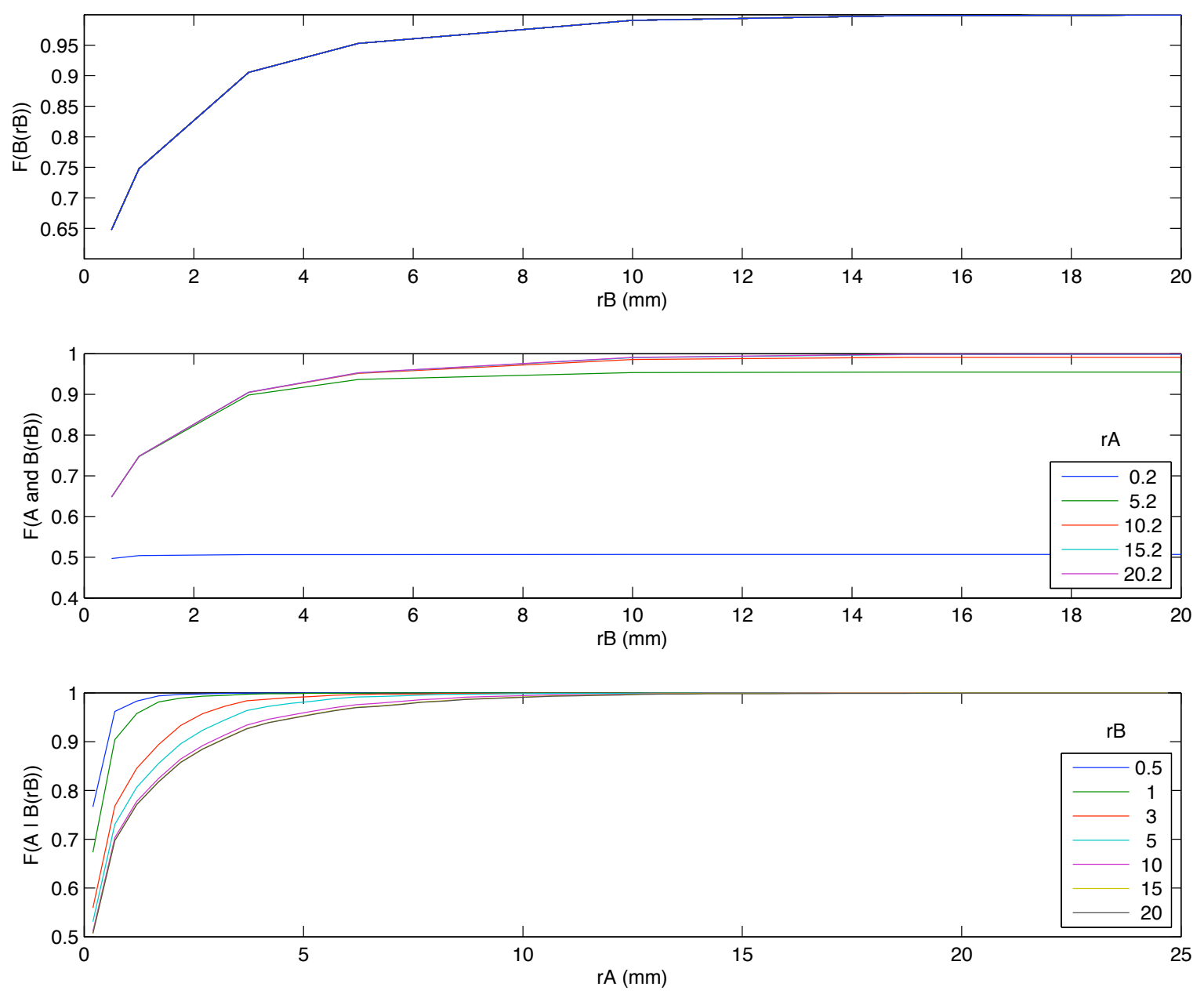

Figure 5.14 Probability distribution functions for the 48-hour lag forecast $(B)$ and the 42hour lag forecast $(A)$ : (a) the probability of $B$ being less than $r_{B}$ as a function of precipitation value $r_{B}$, (b) The joint probability of $A$ being less than $r_{A}$ and $B$ being less than $r_{B}$ as a function of $r_{B}$ for selected $r_{A}$ values, and (c) The conditional probability of receiving less than $r_{A}$ precipitation in $A$ given that less than $r_{B}$ was predicted in $B$ as a function of $r_{A}$.

higher the probability of predicting a very small amount of precipitation in the subsequent forecast. Even a prediction of heavy precipitation $(>15 \mathrm{~mm})$ in the 48-hour forecast has a high probability (0.99) of being below $10 \mathrm{~mm}$ in the subsequent forecast (with 42-hour lag) for the same valid time.

The conditional probability that the subsequent forecast will be under a particular value given the current forecast can be determined for all lags. This conditional probability is shown for a selection of lags in Figure 5.15. To highlight the different curves, Figure 5.15 is also plotted on a log scale in Figure 5.16. It can be seen that 

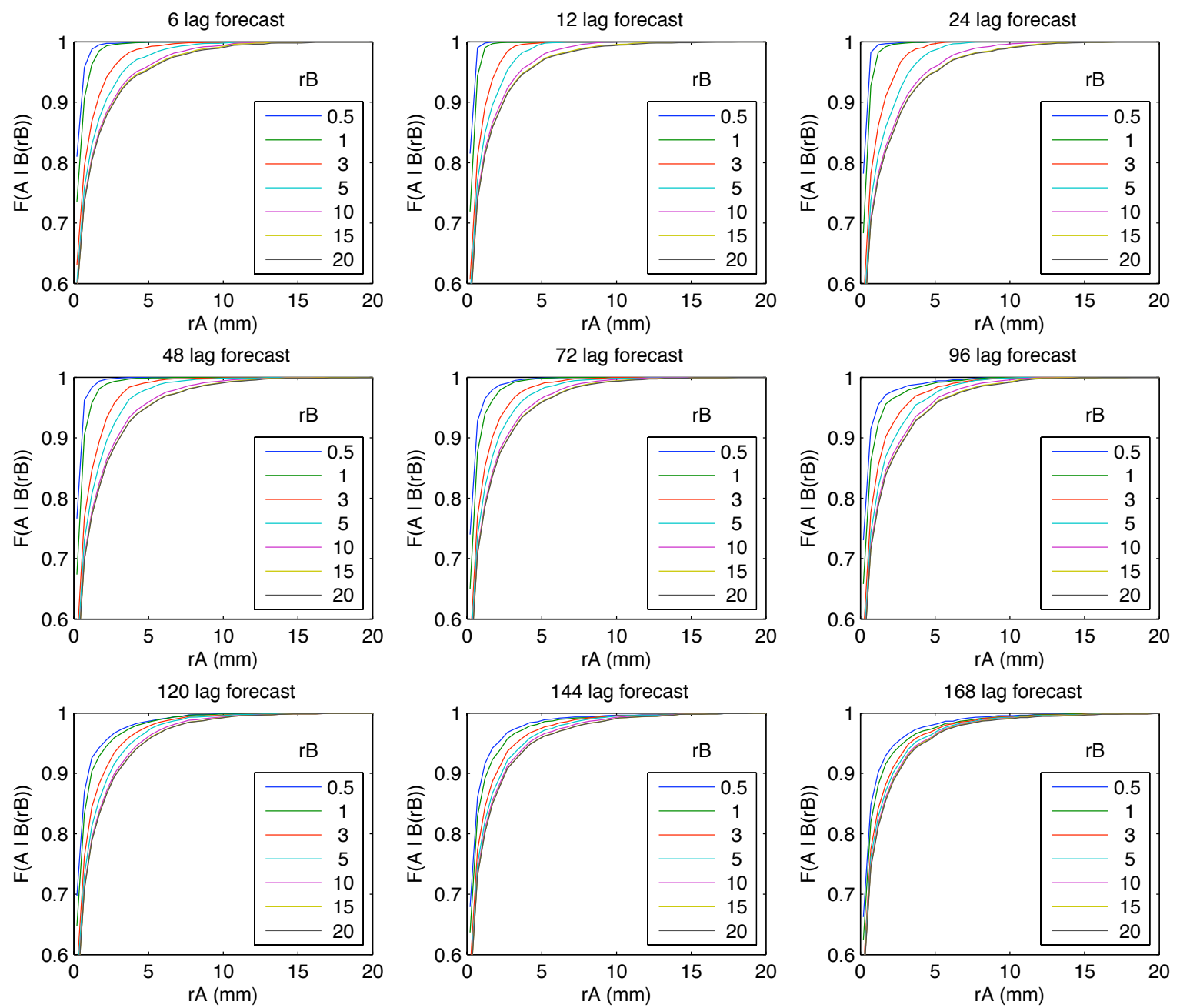

Figure 5.15 Subsequent forecast conditional probability functions for selected lags. The conditional probability of receiving less than $r_{A}$ precipitation in forecast $A$ (at the lags given) given that less than $r_{B}$ was predicted in forecast $B$ (a forecast with the same valid time from the prior model run) as a function of $r_{A}$. The first plot (0-hour forecast) is the conditional probability of receiving actual precipitation given the value in the 6-hour lag forecast.

there is small variations between different lags, although the curvature of the functions do vary.

In the above case, 'A' was the subsequent forecast, however this same theory can be applied directly to the observational data as shown in Figures 5.17 and 5.18. After finding the function that represents the amount of precipitation predicted in one forecast, this can be followed across the graph to determine the probability of predicting less than any amount on the $x$-axis in the subsequent forecast. To determine the maximum amount of precipitation to within a 0.95 probability, a line across the 

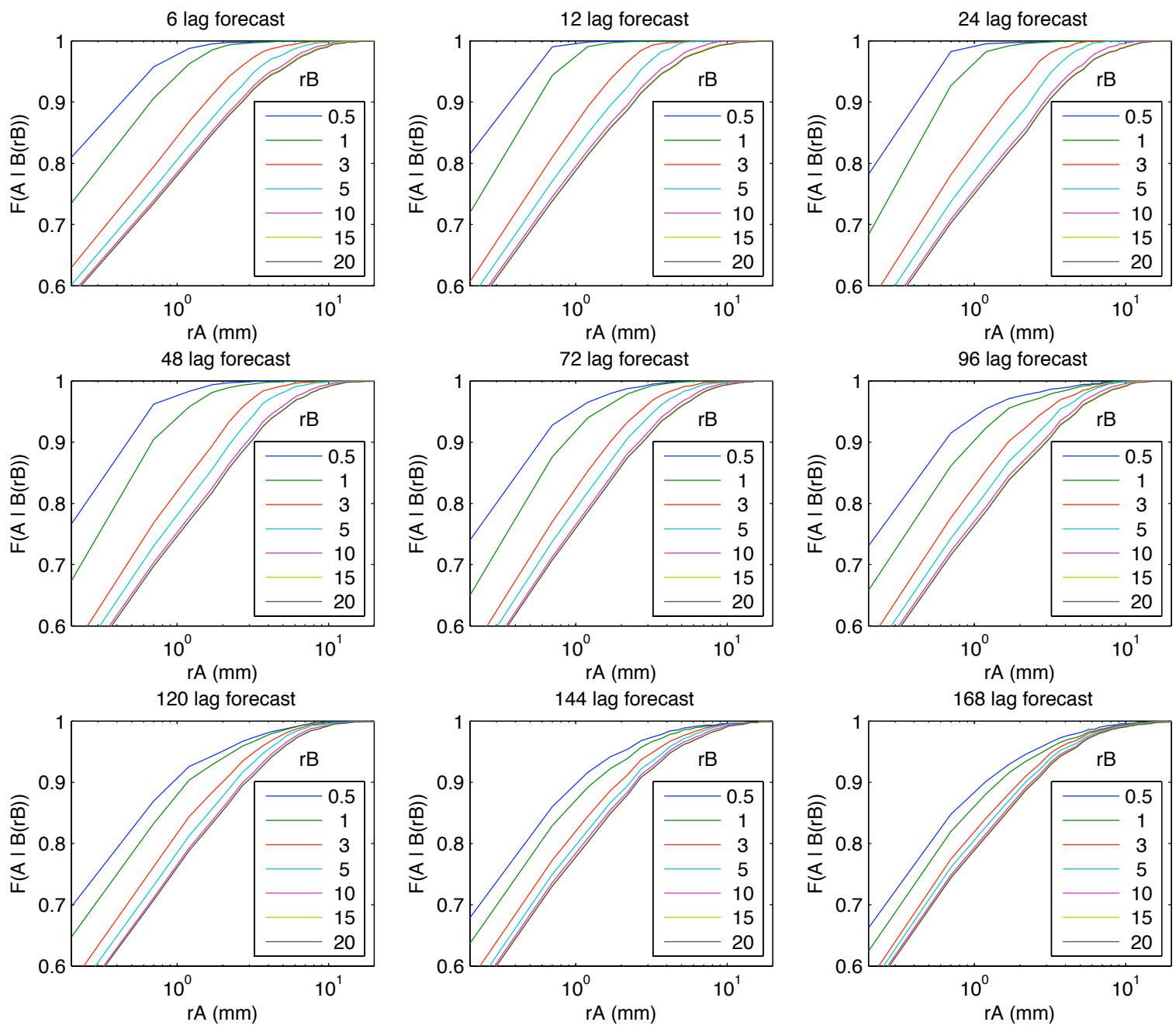

Figure 5.16 Subsequent forecast conditional probability function for selected lags as in Figure 5.15 but with the $\mathrm{x}$-axis plotted in a log scale to highlight the difference in the probability functions.

graph at 0.95 will give us the precipitation value. For example, from Figure 5.17a, after a prediction of $1 \mathrm{~mm}$ of precipitation in the six-hour lag there is a probability of 0.95 of receiving less than $4 \mathrm{~mm}$; but for a $20 \mathrm{~mm}$ prediction, there is a 0.95 probability of receiving less than $7.5 \mathrm{~mm}$.

The real value of this technique is that the higher the probability of the first prediction the greater the probability that the later one is correct. From the curvature of the graphs we can see from Figure 5.15 that the probability distribution functions become steeper for shorter lags. This means that at shorter lags (e.g. a 12-hour lag) a prediction for $1 \mathrm{~mm}$ of precipitation has a very high probability $(>0.97)$ of again 

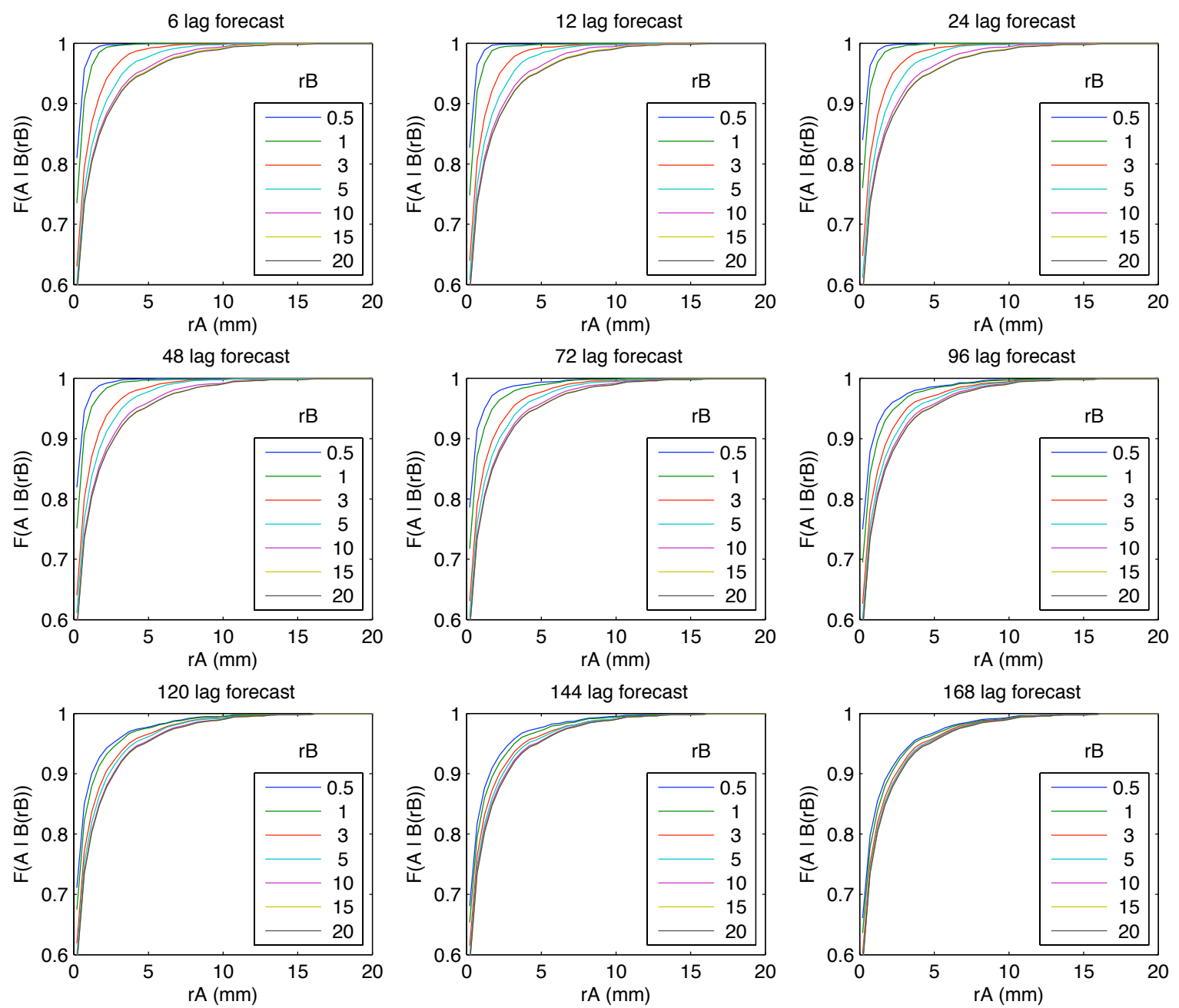

Figure 5.17 Conditional probability function of receiving precipitation given predictions at selected lags. The conditional probability of receiving less than $r_{A}$ precipitation in observations given that less than $r_{B}$ was predicted in forecast $B$ (a forecast with the same valid time from the prior model run) as a function of $r_{A}$. The first plot (0-hour forecast) is the same as in Figure 5.15.

predicting less than $1 \mathrm{~mm}$ of precipitation in the subsequent forecast. However with a 168-hour lag this same prediction sequence only has a probability of 0.88 .

Through both the comparison with subsequent forecast and observations (Figure $5.19)$, it can be seen that the conditional probability of receiving $1 \mathrm{~mm}$ given a forecast of $0.5 \mathrm{~mm}$ increases at shorter lag. However, in this case, the probability of observing less than $1 \mathrm{~mm}$ if $1 \mathrm{~mm}$ was forecast at 12-hours lag is only 0.86 , much less than the 0.99 probability of predicting the value in the next forecast. Predictions of $>15 \mathrm{~mm}$ have very little variation regardless of lag (Figure 5.19). This is due to the relative 

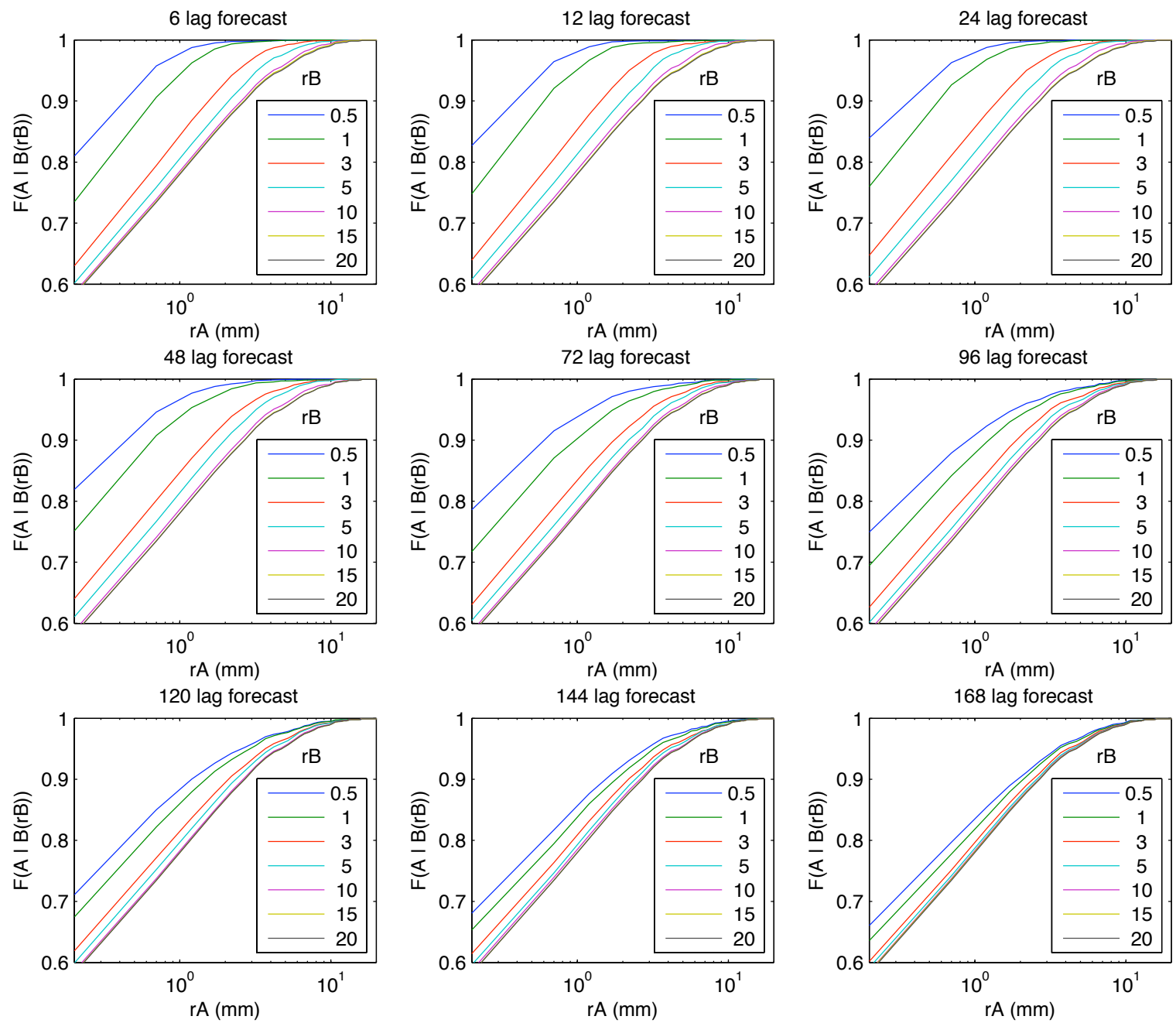

Figure 5.18 Conditional probability function of receiving precipitation given predictions at selected lags as in Figure 5.17 but with the x-axis plotted in a log scale to highlight the difference in the curvature of the probability functions.

infrequency of these values in forecasts or observations $(\operatorname{Pr}(X>20)=0.006)$.

In general, this means that the probability of receiving less than $20 \mathrm{~mm}$ of precipitation in either the subsequent model run or in the observations is not as affected by the lag. However, for smaller amounts of precipitation the probability of forecasting a similar amount in the subsequent forecast increases as the lag is reduced. This increased probability with shorter lag is not as noticeable when the forecast is compared to observations, but again there is a higher probability of correctly predicting these amounts at shorter lags. 

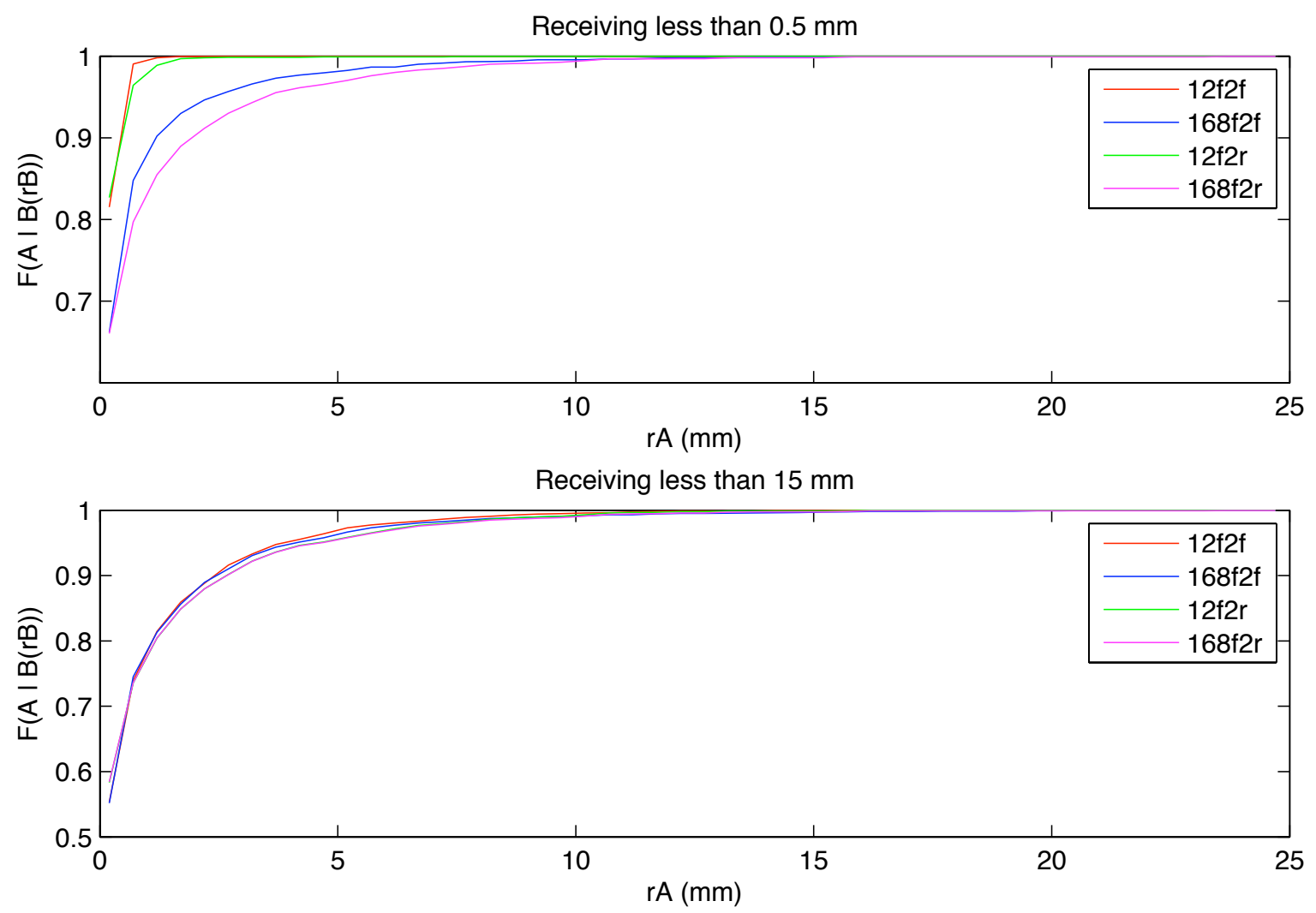

Figure 5.19 Comparison of conditional probability functions for receiving specified precipitation amounts. (a) The conditional probability of receiving less than $0.5 \mathrm{~mm}$ of precipitation; i) in the 12-hour forecast given the $r_{B}$ value in the previous forecast (identified as 12f2f), ii) in the 168-hour forecast given the $r_{B}$ value in the previous forecast (168f2f), iii) observing it given the $r_{B}$ value in the 12-hour forecast (12f2r), and iv) observing it given the $r_{B}$ value in the 168-hour forecast (168f2r). (b) The same as (a) but for receiving less than $15 \mathrm{~mm}$ of precipitation.

Using the same idea, the probabilities can be weighted by the number of times that the situation has been previously predicted. The two categories $>1 \mathrm{~mm}$ and $>10 \mathrm{~mm}$ are shown in Figure 5.20. In this figure, the conditional probability of precipitation above the threshold being in a forecast is calculated conditional on the number of previous forecasts that exceeded the same threshold. This clearly shows that if previous forecasts have predicted precipitation then the probably that it will predict precipitation in the current model run is increased. However, this relationship is stronger for the precipitation category of $>1 \mathrm{~mm}$ than for the heavy precipitation category. Due to the limited number of observed events (especially of heavy precipitation) there are many possibilities that did not occur, or only occurred once during our time period, 

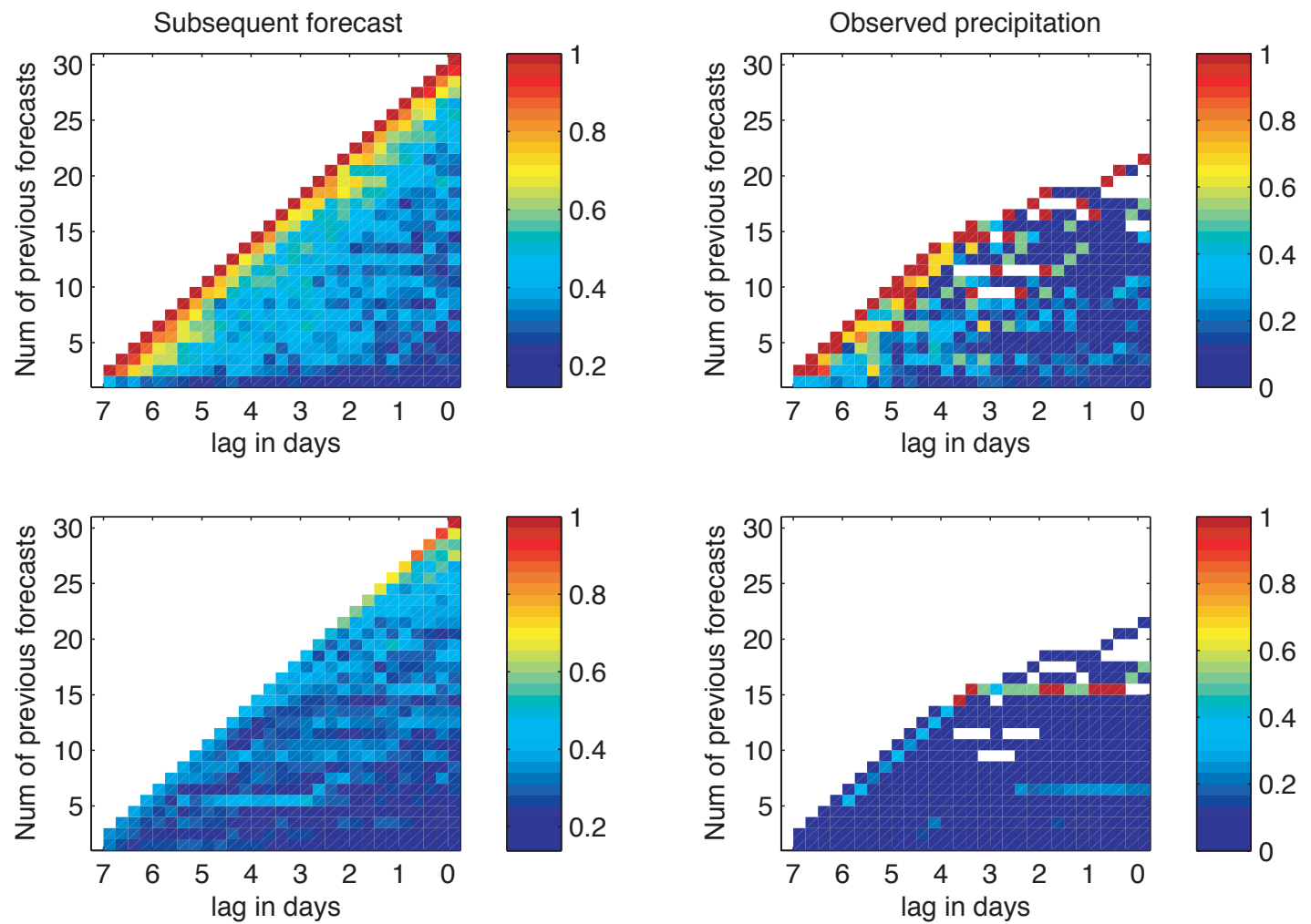

Figure 5.20 The conditional probability of receiving precipitation over a threshold dependant on the number of previous forecasts for that valid time that have exceeded the threshold. The top line is for a threshold of $1 \mathrm{~mm}$ and the bottom line for a threshold of $10 \mathrm{~mm}$. On the left is the probability of receiving precipitation in the next forecast and on the right is the probability of observing the precipitation totals.

and these results are less robust, but a similar relationship is seen with a conditional probability calculated when more previous model runs have predicted the precipitation.

\subsection{Discussion}

The GFS model is valuable as an extended range forecast because it is skilful at predicting fine weather and indicating the presence of a precipitating mesoscale feature although the features are not well constrained at the longer lags. The improved accuracy at the shorter lags suggests that this model is appropriate in most cases for initialising and constraining the mesoscale model, which can determine the surface interactions and therefore reduce the under-prediction of heavier precipitation found here. 
The hit rate, mean error and root mean squared errors calculated in this chapter indicate that the GFS model has value in predicting precipitation values, but all of these measures are influenced by the abundance of dry periods. However, the probability of detection, false alarm rate and skill score all showed poor accuracy. Bias scores and mean errors show an under-prediction in the six-hour forecast, which can be accounted for by model spin-up and then a general over-prediction of precipitation. The bias scores also showed that light precipitation was over-predicted and heavy precipitation was under-predicted. This could result from a lack of orographic enhancement at the resolution of the model. However, this detailed enhancement can be corrected through the mesoscale modelling (as described in Chapter 6).

The large individual errors and small area averaged error indicate that the amount of precipitation reaching the area is reasonably well predicted while the exact location of the precipitation is less accurate. There is a tendency to over-predict precipitation in the northwest and within the Waikato River catchment and around the coastlines. The locations that had the largest errors were also the stations that had a larger standard deviation in the range of errors.

Ensemble analysis showed that the probability of receiving heavy precipitation increased as the number of ensemble members predicting an extreme increased. This ensemble idea was extended to using probability distribution functions to show that specific precipitation predictions in a forecast could be related to observing precipitation below a particular value. The probability of receiving the same value in consecutive forecasts was increased with decreased lag. Similarly, the probability of predicting observed precipitation increased as the lag decreased. These results are consistent with those in Gallus et al. (2007).

The ensemble analysis showed that some of the error could not be accounted for by the low resolution of the global model. In some model runs, the mesoscale meteorological features were in different locations, while in other runs, the precipitation features did not develop. The largest variations are more likely to occur at longer lag where 
the model would have had more opportunities to deviate. As high-resolution mesoscale models are constrained by the global model output this means that these large-scale errors will be propagated to any high-resolution forecasts. The time lag ensemble also showed that the probability of precipitation increased with the more members that predicted precipitation.

The following chapters will look at the ability of the mesoscale model to simulate the weather given 'accurate' constraints, but the lag ensemble and probability distribution functions shown here, show the uncertainty in these input conditions. The GFS model now provides a 17 -member ensemble and spread out to 16 days ${ }^{\S}$ that will provide uncertainty estimates in the model at longer lags where a time lag ensemble currently contains few members. The 16-day, 17-member ensemble data were unfortunately not available for the time period used here. If large-scale forecasts are improved in the future, substantial quantitative precipitation skill should be gained at all resolutions.

\footnotetext{
$\S$ http://www.emc.ncep.noaa.gov/gmb/ens
} 
CHAPTER 6

\section{MESOSCALE SIMULATIONS}

Chapter 5 showed that while the general mesoscale features of a weather system can be predicted four days in advance, the global model does not have the resolution to simulate the interaction of the weather system with the land mass and therefore predicts more evenly distributed lighter precipitation throughout the region than what is observed. A mesoscale model, such as the Weather Research and Forecasting (WRF) model, can be used to simulate the predicted weather system at higher resolution over a limited domain and include the land/air interactions. Mesoscale models have been used to increase forecast resolution from 1 degree to $1 \mathrm{~km}$; however, the run time for the model is dependent on the number of grid points so there are practical trade-offs between domain size and resolution. Public daily weather forecasts in New Zealand currently use mesoscale models run at a 10-12 km resolution.

Topographical features surround the Waikato River catchment, and as seen in Figure 3.2 there can be significant differences in the precipitation received at locations that would be within a single grid-square of the global model. For the purpose of estimating river flows it is important to know the distribution of precipitation, and thus whether the precipitation will be inside the catchment area or in the surrounding areas. The mesoscale simulations discussed in this chapter illustrate the improvement in precipitation predictions made by increasing the resolution of the model. This chapter also investigates the mechanisms for precipitation generation within the model and looks at the abilities and limitations of the mesoscale model in simulating precipitation through a series of high-resolution simulations of three heavy precipitation events. 
Testing the accuracy and limitation of numerical weather models through the simulation of heavy precipitation events has been around at least since the cyclone simulation in Baer and Boudra (1977). Numerical models have significantly advanced since that 1977 study, however, especially in terms of the representation of boundary layer physics, initialisation data and procedures and boundary conditions.

Understanding the development of a particular weather system was the main aim of Mölders (2008), Colle (2003), Milbrant and Yau (2001), Colle and Mass (2000), Buzzi et al. (1998), Katzfey (1995, a New Zealand study), Sinclair (1993, a New Zealand study), Hess (1990), Kuo et al. (1988), Kalb (1985) and many more. All of these papers examine devastating storms where the operational forecasts had failed to predict the observed quantity of precipitation. Many of these studies led to recommendations for and subsequent implementation of model improvements.

Many further studies have also used numerical simulations of extreme precipitation events to explain the development of a weather system, test the model's sensitivity to physics schemes, and test the resolution required to simulate the event. The WRF model has been available since 2003. It is the successor to the MM5 (Fifth-generation NCAR/Penn State Mesoscale Model) model that was used for the majority of mentioned literature. Recent studies, e.g. Mölders (2008) and Zängl (2007), have used the WRF model, and Chang et al. (2008) showed that the WRF model was superior to the MM5 at modelling precipitation in a 2005 heavy rain event over Mumbai, India.

For this study, three precipitation events that occurred during the two years included in the GFS archive were simulated on a three-km grid using the WRF model. In this study, the run time of the simulation was less important and therefore higher resolutions than operationally practical could be used to investigate the impacts of resolution on the simulation of precipitation. As previously mentioned orographic enhancement affects precipitation distributions within New Zealand. The higher resolution mesoscale model means that more detailed topographic and land use information can be included and more realistic precipitation distributions potentially can be 
achieved. For each of the events, a complete simulation was conducted as well as a simulation that excluded all topography to quantify the orographic enhancement effect within the Waikato River catchment.

To examine the mechanisms of precipitation generation in the model and test the effects of the different physics options available in the WRF model eight additional simulations were conducted for the largest event. Aspects of the precipitation field still have to be parameterised in high-resolution models as precipitation develops on a micrometre scale, which is still beyond the practical limitations of computing systems over a domain this size. Examining the sensitivity of the model physics was the motivation for studies by Zängl (2007), Gallus and Bresch (2006), Colle et al. (2005), Grubišić et al. (2005), and Wacker (1995) and others. Many of the identified limitations involved with the physics (microphysics, cumulus parameterisations, dynamic cores etc.) have since been improved in the respective model physics. Precipitation in the model output is in two fields; the 'resolved' precipitation that is dynamically forced by the microphysics scheme and the 'convective' precipitation that is generated from the cumulus parameter. Convective precipitation is a sub-scale process and is parameterized so the timing and location of this precipitation is not physically constrained in the model but must occur to keep the water balance of the atmosphere stable.

\subsection{The Weather Research and Forecasting model}

The WRF model (version 2.1) is a numerical mesoscale weather model developed at NCAR and NCEP that can be used over a limited area for simulation of a weather sequence or to produce an operational weather forecast. In either case the model requires global model output to initialise and constrain the domain boundaries and numerous user defined model settings. These settings include the output data required, the domain boundaries, and which physics schemes are used to represent the planetary boundary layer, radiative, convective and microphysical processes.

The model is freely available and comes with a default namelist, which details the 
options used operationally by NCEP. Documentation on these options is provided in Skamarock et al. (2007) and the papers referred to within. Within the domain boundaries, the physical parameters such as topography, vegetation and soil type are provided in the accompanying software called WRFSI (Weather Research and Forecasting Standard Initialisation), and more recently upgraded to WPS (the WRF Pre-processing System). These domain properties are provided at 30 seconds of arc resolution and world sections can be downloaded with the model.

Increasing the resolution of the model will generally increase the model's skill at quantitative precipitation forecasts and produce a more realistic precipitation structure (Colle, 2003; Colle et al., 2000, illustrated by a comparison between 36-km and four-km grids). Colle et al. (2005) considers a 10-20\% error in precipitation forecasts as good, but also warns that even if the quantity of precipitation is well simulated, the water phase or cause of the precipitation may still be wrong. These errors arose from the model's ability to handle low-level atmospheric ice in the Wasatch Mountains (USA), the topography in this study is much lower and therefore this effect should have less impact. This study illustrated the potential for the model to be accurate in the quantity of precipitation, but not the processes creating it, which will make the accuracy of the model dependent on the processes involved in the development of precipitation.

\subsubsection{Control model settings and parameters}

The same model settings were used in each of the three heavy precipitation events modelled in this section. Each model run is controlled by a namelist.input file that lists which options are to be used for the run. The complete namelist.input file for the January 2006 Control simulation is provided in Appendix C. A summary of the most important parameters (in this study) is shown in Table 6.1.

Generally, in an operational setting the WRF model is initialised and constrained along the boundary by data from a global forecast model run. As the simulations presented in this chapter have the advantage of hindsight, the variation in simulation 
Table 6.1

The WRF settings for each high-resolution Control run simulation

\begin{tabular}{|l|l|}
\hline Parameter & Setting \\
\hline \hline Initialisation and forcing & GFS analysis 1 degree model analysis \\
\hline Vertical levels & 27 \\
\hline Grid spacing & $27,9,3-\mathrm{km}$ \\
\hline Core & Advanced mass dynamical core \\
\hline Microphysics & Thompson \\
\hline Cumulus physics & Kain-Fritsch in outer two domains \\
\hline Surface physics & Noah land-surface model \\
\hline Planetary Boundary Layer Physics & Mellor-Yamada-Janjic \\
\hline Topography & Standard from WRFSI scheme \\
\hline Surface wetness & full soil model \\
\hline Time Step & 180 seconds \\
\hline Feedback & Off \\
\hline Smooth option & on \\
\hline nesting & two-way \\
\hline
\end{tabular}

length, and the uncertainties associated with a single global forecast run, a single GFS run was not used to initialise and constrain these simulations. The analysis files (data assimilation of real-time global observations) for each global model run during the period of the event were compiled to form 'perfect' boundary conditions. Therefore the performance of these simulations should be the upper limit of the achievable skill using these configurations in operational forecasting.

All these model runs used three nested domains, and unless stated otherwise are as described here. The outer domain is a $27-\mathrm{km}$ grid centred on the point $175^{\circ} \mathrm{E} 38^{\circ} \mathrm{S}$, and consists of 78 grid points in the east-west direction and 75 in the north-south direction as shown in Figure 6.1. The topography, soil, and vegetation parameters were calculated for each grid square by the WRFSI program during the initial set up of the model domains. The topography of New Zealand at this resolution is also shown in Figure 6.1 that shows substantially smoothed topography over the Waikato river catchment at a $27-\mathrm{km}$ resolution.

The first nested domain, middle, is a nine-km grid centred on the same point and 


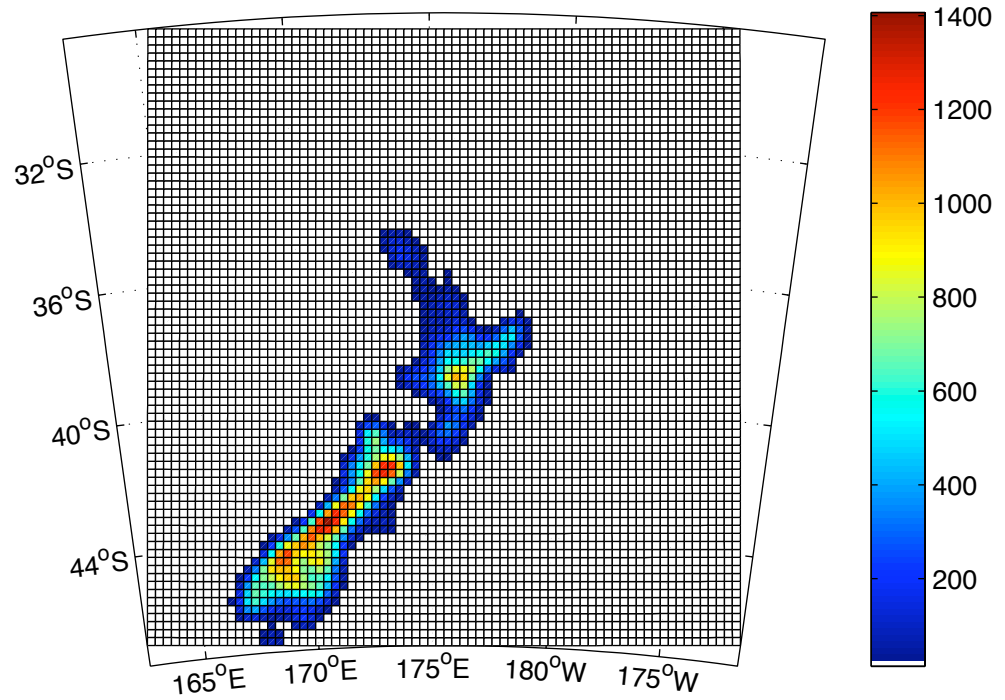

Figure 6.1 High-resolution WRF model simulation 27$\mathrm{km}$ outer domain grid and surface topography.

also has 78 grid points in the east-west direction and 75 in the north-south direction as in Figure 6.2. Using the general rule of thumb from the modelling community, the

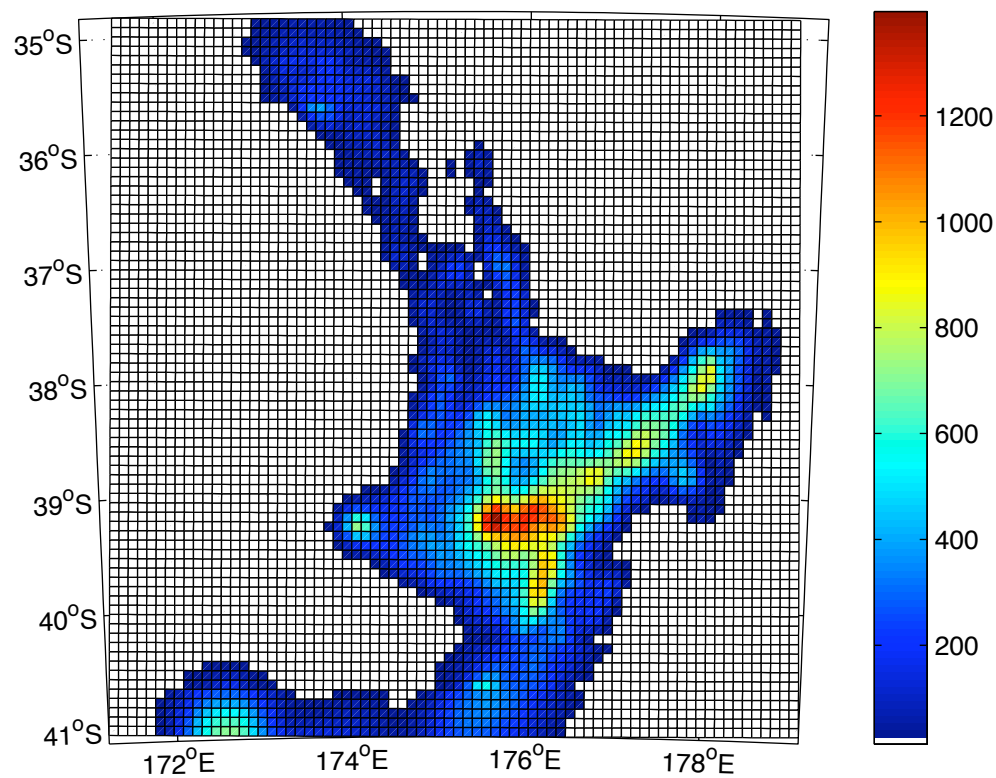

Figure 6.2 High-resolution WRF model simulation nine- $\mathrm{km}$ middle domain grid and surface topography.

resolution increases by a factor of three between nests. The inner domain, is a three$\mathrm{km}$ grid centred on the same point and has 99 grid points in the east-west direction and 93 in the north-south direction as in Figure 6.3. The Waikato River catchment is in the southeast quadrant of the inner domain to include more of the incoming system, which generally arrives form the northwest. These three domains are two-way nested meaning that information from the inner domains is fed back to the outer domains, 


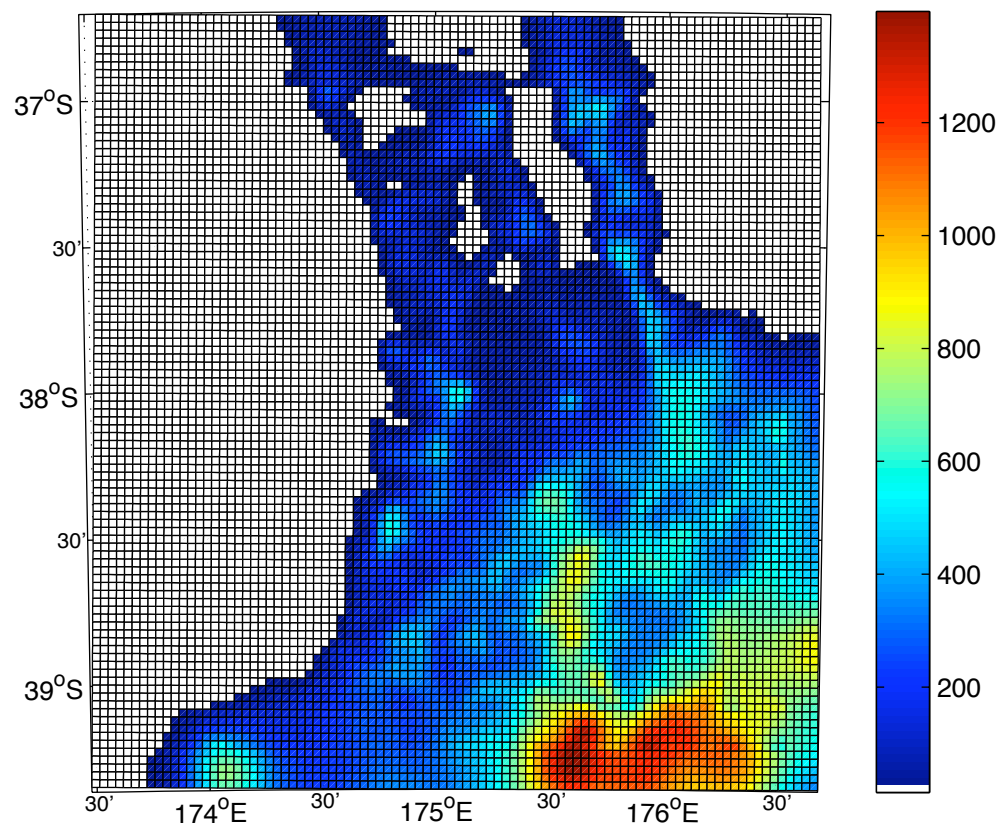

Figure 6.3 High-resolution WRF model simulation three-km inner domain grid and surface topography.

and weather systems develop simultaneously in all domains.

Further increases in horizontal resolution would require significantly more grid points and run time and are unlikely to result in significant improvements for the precipitation forecasts (Colle and Mass, 2000, this was shown between their four-km and $1.3-\mathrm{km}$ domains). The general rule is that doubling the resolution increases the run time by a factor of eight. At the current settings described above each model run took on average six days on the available Linux server*.

The vertical resolution was set to 27 vertical sigma levels for all domains. Sigma levels (see equation 6.1) follow the shape of the surface and range between zero and one:

$$
\sigma=\frac{p}{p_{0}}
$$

where $p$ is pressure and $p_{0}$ is the surface pressure, so $\sigma=1$ is the surface and $\sigma=0$ is the top of the atmosphere. The 27 levels used in this study are shown in Figure 6.4. Pressure varies logarithmically with altitude and most precipitation occurs in the lower reaches of the atmosphere so model levels are denser nearer the surface.

\footnotetext{
*a 2GHz dual-core Opteron with 4GB RAM.
} 


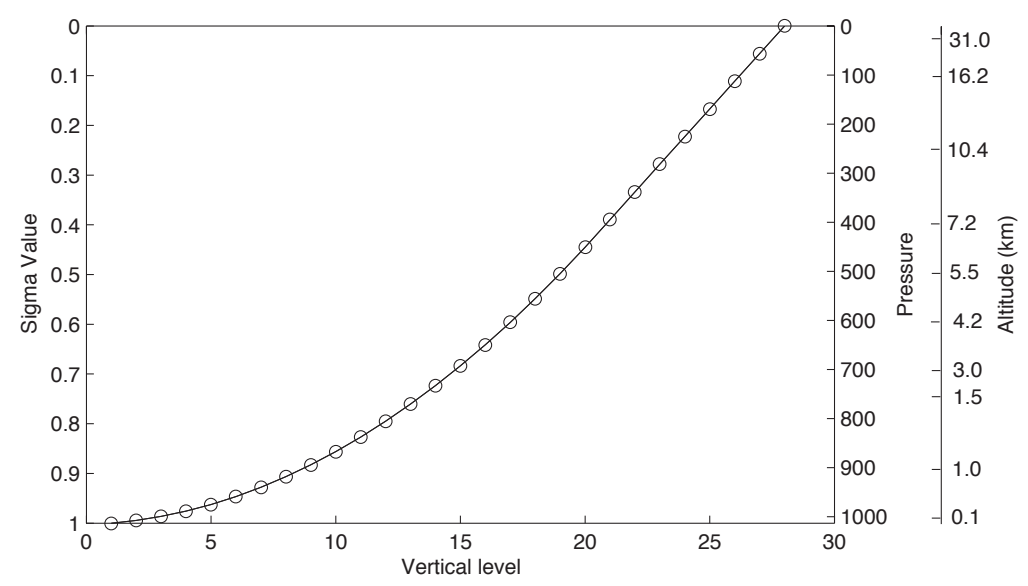

Figure 6.4 The 27 vertical sigma levels used for all domains in the high-resolution WRF simulations. The right-hand axis shows the corresponding pressure levels and approximate altitudes according to a standardised atmosphere where the surface pressure is 1013 mb (Holton, 2004).

The microphysics scheme determines how the model calculates the small-scale features such as cloud development and precipitation. The Thompson microphysics (Thompson et al., 2004) scheme was chosen for the 'Control' model run as it was specifically developed for mid-latitude locations where precipitation is driven by baroclinic waves. These were identified in Chapter 4 as a main cause of heavy precipitation in the Waikato River catchment.

Convective precipitation usually occurs over a much smaller area and therefore is calculated from a cumulus parameter. The Kain-Fritsch parameterisation (default setting) was designed to simulate vertical moisture movement and its trigger is based on grid-resolved vertical motion (Kain and Fritsch, 1990; Kain and Fritsch, 1993). This parameterisation scheme has been updated and the modified Kain-Fritsch scheme (Skamarock et al., 2007, page 55) was used. The scheme was modified to deal with entrainment issues identified during testing within the Eta model.

\subsection{Case studies}

To illustrate the improvement in forecasting precipitation distribution between the global and mesoscale models, three heavy precipitation events were chosen for mesoscale simulation. The characteristics of these three events are typical of heavy precipitation events in the Waikato River catchment (see Chapter 4, the January 2006 event was chosen as a case study in both chapters so the results could be compared), and should 
test the model over the variety of precipitation mechanisms. Two of the three chosen events exceed the 95th percentile of the regional time series (see Figure 3.7) and are the two largest events that occurred during the GFS data collection period. The third event, October 2006, did not exceed the threshold but was included to investigate why operational forecasts had over-predicted the precipitation (which had led to numerous weather warnings being issued by the MetService).

The three events were characterised by:

- The rapid sequence of two mid-latitude cyclones entraining unstable tropical air resulting in localised heavy showers and thunderstorms in October 2005, representing a period of enhanced baroclinicity;

- The closely timed arrival of an intense subtropical cyclone and the cold front from a Southern Ocean mid-latitude cyclone in January 2006 producing the largest daily precipitation peak during the period of initialisation data; and

- The development of a slow moving, blocked, mid-latitude cyclone in the Tasman Sea in October 2006, representing the blocking conditions often associated with heavy precipitation.

Each event was simulated twice, first using the 'Control' settings in Table 6.1, and then with all topography scaled to sea level to quantify the expected orographic enhancement effect (referred to as the 'NoTopo' simulations).

The spatial distribution of the precipitation climate (see Figure 3.2) mimics the topographic distribution; de Lisle (1967) recognised that topographic features were influencing the distribution of precipitation within the Waikato River catchment. A similar idea of scaling topography was used to investigate the orographic forcing in Milbrant and Yau (2001), Colle and Mass (2000), and Buzzi et al. (1998). The 'NoTopo' model runs are unrealistic but show how topographic features influence the precipitation distribution within an area, and indicate the required resolution to correctly simulate the orographic enhancement in an event. 
For each case study, selected MetService analysis maps and MTSAT satellite images are provided to show the synoptic development. Additionally, the regional precipitation time series (as in Chapter 4) and hourly meteorological observations (at stations shown in Figure 6.5) are also provided.

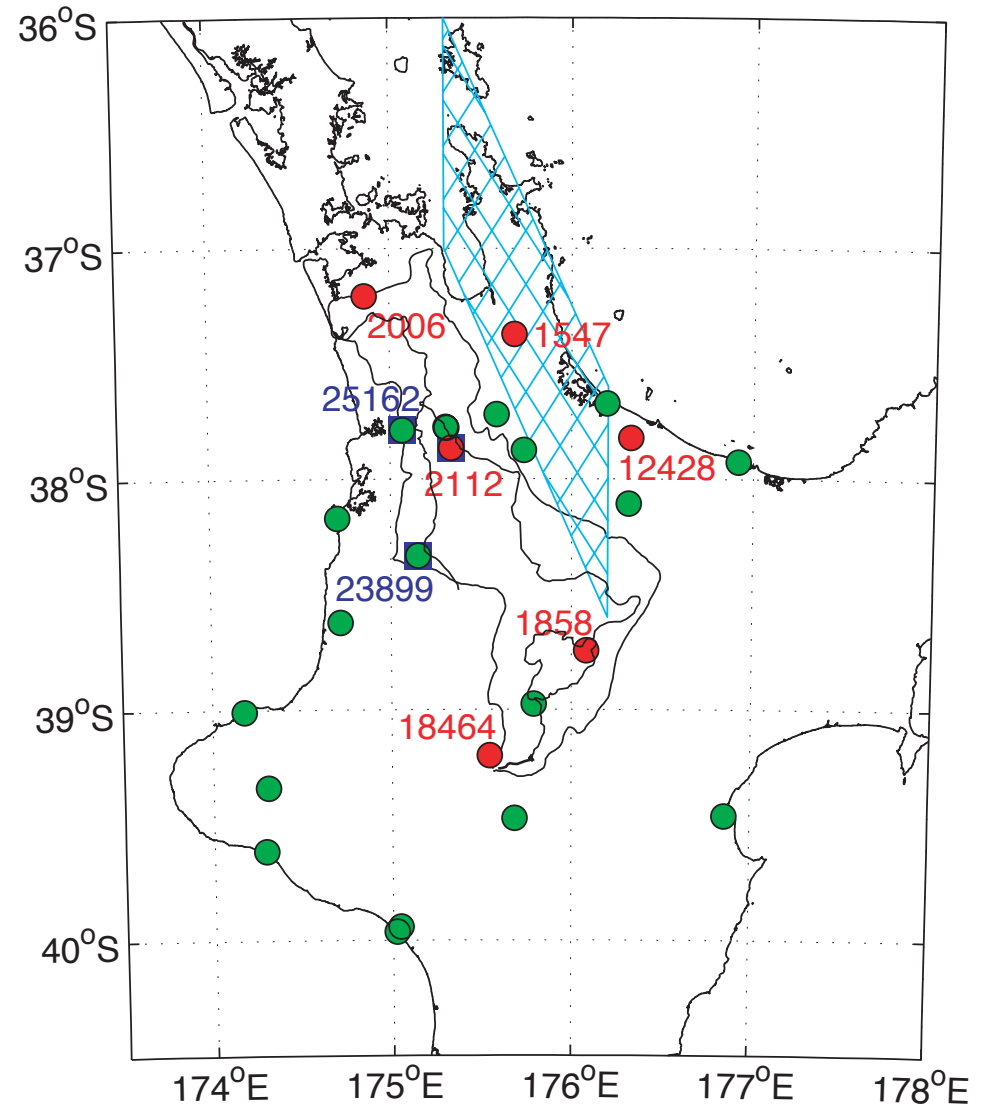

Figure 6.5 The locations of observation stations mentioned in this chapter. Green and red dots represent the hourly rain gauges throughout the region. The red dots signify the rain gauges that are used for time series comparison in Figures 6.30, 6.37, 6.41, and 6.44. Its agent number identifies each of these gauges. The squares represent the locations of the pressure and temperature gauges used in Figures 6.9, 6.17, and 6.25. The hatched parallelogram represents the area where topography was altered in the 'Kaimai' simulation mentioned in section 6.3.1.

The model runs were initialised from the analysis fields and not the six-hour lag forecasts, but as no precipitation exists in the analysis, the six-hour forecasts are shown to approximate the precipitation field according to the GFS model during the event. The combined convective (generated through the cumulus parameterisation) and resolved (generated through the microphysics scheme) precipitation from the mesoscale model's outer domain is also plotted in six-hourly accumulations for comparison.

The total accumulated precipitation during the event was also calculated at each rain gauge station, over each GFS grid square, and in the 'middle' domain for both the Control and NoTopo model simulations. Regional hourly precipitation time series 
were also calculated from the observations inside the catchment and the modelled precipitation split into resolved and convective precipitation (using data from the middle domain as the different precipitation types are not differentiated in the inner domain). All of these are provided for each event for completeness.

\subsubsection{October 2005}

During early October 2005 there was a period of unusually warm unsettled air entrained between the passages of two mid-latitude cyclones. During this period there were heavy showers, strong westerly gales and thunderstorms (McGavin, Quarterly Newsletter). There were no reports of damage in the Waikato due to the weather sequence but there was a large power cut and roofs were blown off houses in Auckland on 8 October, and 11 October brought floods to the Gisborne region. However, precipitation totals were significantly high exceeding the 99th percentile of the smoothed regional time series and the 98th percentile of the daily regional time series.

\section{Event observations}

On 10 October there was a daily precipitation peak of $22.3 \mathrm{~mm}$. The area averaged smoothed time series peaked at $15.2 \mathrm{~mm}$, on 9 October (Figure 6.6). Moderate per-

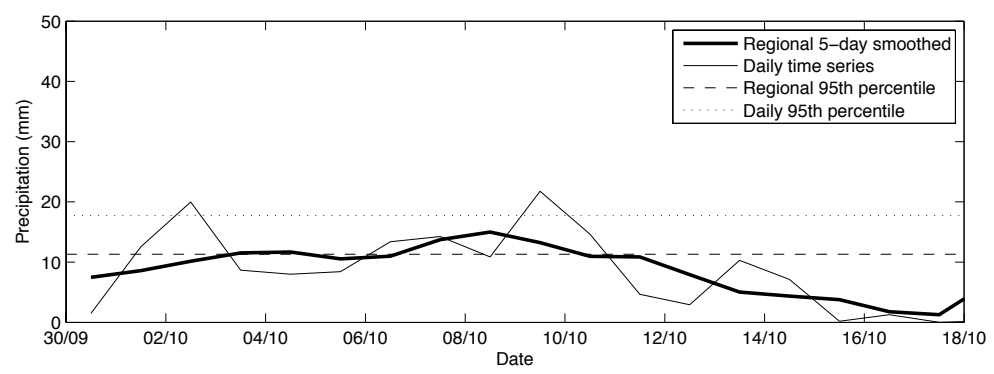

Figure 6.6 Daily precipitation time series for the October 2005 event. The thick solid line is the regional area-average smoothed time series. The thin solid line is the daily area-average time series. The dashed line shows the 95th percentile of the smoothed time series and the dotted line represents the 95 th percentile of the daily time series.

sistent precipitation occurred for the week prior to the event including another peak 
(above the 98th percentile of daily precipitation at $16.7 \mathrm{~mm}$ ) on 2 October that would have saturated the area and made it more prone to flooding.

The MetService analysis maps (Figure 6.7) and satellite images (Figure 6.8) show the passage of the two mid-latitude cyclones across the South Island of New Zealand.

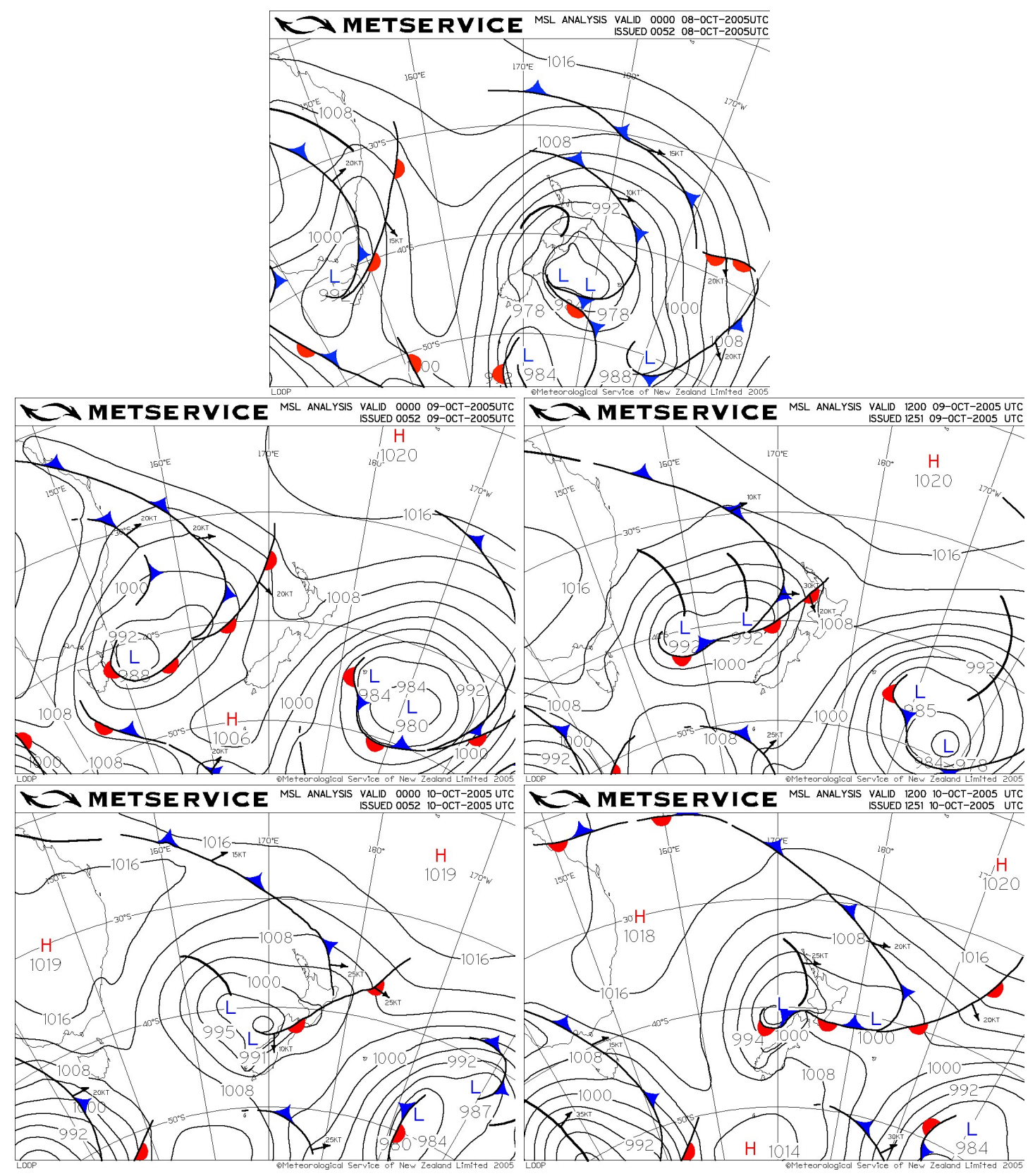

Figure 6.7 Selected MetService analysis maps for the October 2005 event. These maps are valid for: 0000 UTC 8 October; 0000 UTC 9 October; 1200 UTC 9 October; 0000 UTC 10 October; and 1200 UTC 10 October.

The analysis map for 0000 UTC 8 October shows the slow moving mid-latitude cyclone 


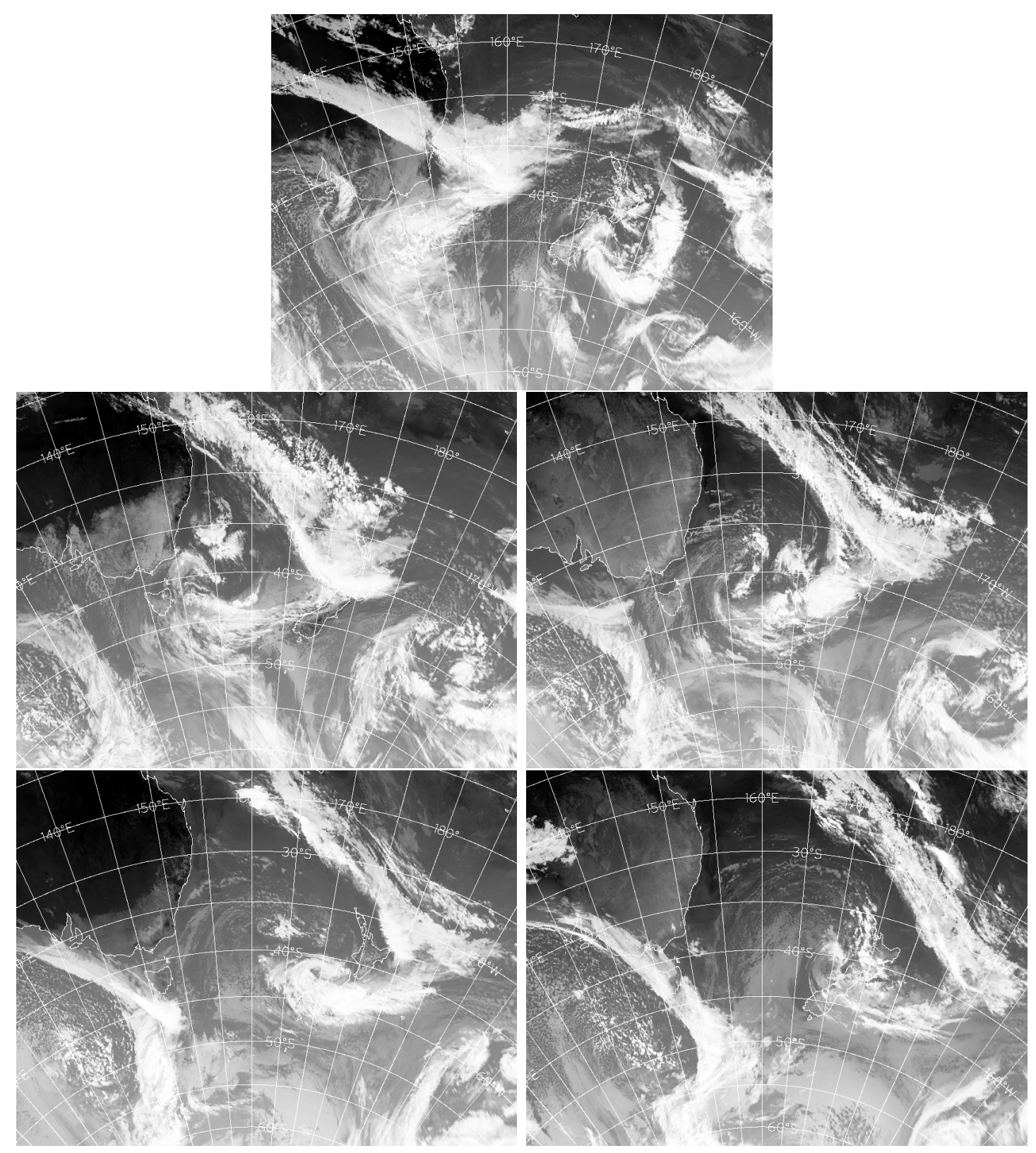

Figure 6.8 Selected infrared MTSAT satellite images provided by the MetService for the October 2005 event. These maps are valid for: 0000 UTC 8 October; 0000 UTC 9 October; 1200 UTC 9 October; 0000 UTC 10 October; and 1200 UTC 10 October.

east of New Zealand and the initial cyclogenesis of the second mid-latitude cyclone near Australia. The associated frontal bands from the first cyclone produced precipitation over the Waikato River catchment on 7 October. The second mid-latitude cyclone entrained more tropical air that sustained thunderstorms (see satellite images) and led to the flooding in Gisborne on 10 October. 
Hourly observations of the atmosphere (Figure 6.9) show that there were small re-
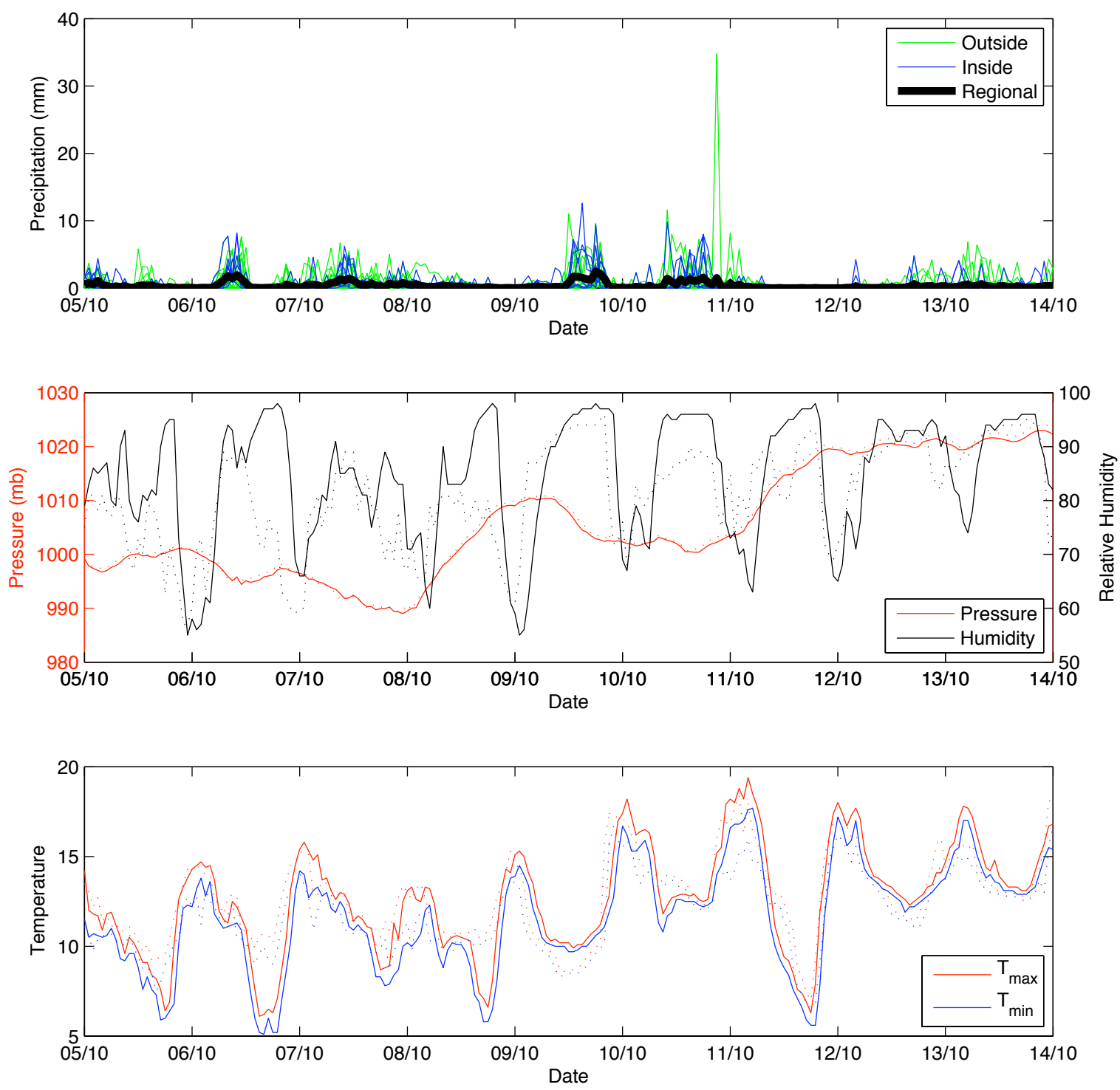

Figure 6.9 Hourly meteorological observations for the October 2005 event. (a) The hourly precipitation time series. Blue lines represent stations inside the river catchment boundary and the green lines are those outside. The thick black line represents the average time series of the blue lines. Pressure is recorded at stations 2112 and 23899 (dashed line) and relative humidity and temperature are recorded at stations 23899 and 25162 (dashed line) (shown in Figure 6.5). Subplot (b) shows the average pressure (red) and relative humidity (black) throughout the event, and (c) shows the maximum (red) and minimum (blue) temperatures each hour throughout the event.

gional hourly precipitation totals indicating that precipitation was localised. However, there were multiple periods of intense scattered showers on $6,7,9$, and 10 October, which would have accumulated to the total values seen in Figure 6.6. 
The hourly observations of temperature, humidity and pressure all clearly show a diurnal signal (Figure 6.9b and c), which have been included for completeness and understanding of the large-scale processes. However, there is a general increase in pressure and temperature over the whole event, with the second cyclone caused a small dip in pressure as it passed. The diurnal signal in temperature and humidity were dampened during the precipitation periods (of all events).

The largest total accumulated precipitation volumes (Figure 6.12a) were observed along the west coast, the Coromandel and on the volcanoes. This map shows that there were large variations in the amount of precipitation received at different observation gauges that are geographically close. Some of these, e.g. Mt Ruapehu, could be a result of large local topographical differences. However, topography cannot account for the $150 \mathrm{~mm}$ difference between two nearby gauges on the west coast. Therefore, topography cannot be the only factor influencing the distribution of precipitation in this event.

\section{Global model data}

The GFS data for this period (Figure 6.10) show that the global model identified the decaying mid-latitude cyclone to the east of New Zealand on 8 October. Then a ridge brings subtropical air into the Tasman Sea. The second mid-latitude cyclone edged into the domain at 1200 UTC on 8 October. During the early stages of development the GFS model predicted precipitation to occur west of New Zealand that continued onshore by 1800 UTC the following day. The pressure minimum continued to deepen in this location until it passed northeast over the North Island at 0000 UTC on 11 October. The GFS model predicted up to $20 \mathrm{~mm}$ of precipitation along the west coast of the North Island (Figure 6.12), as noted in Chapter 5, this was significantly less than what was observed by rain gauges in the area (observing between 50-150 mm). 

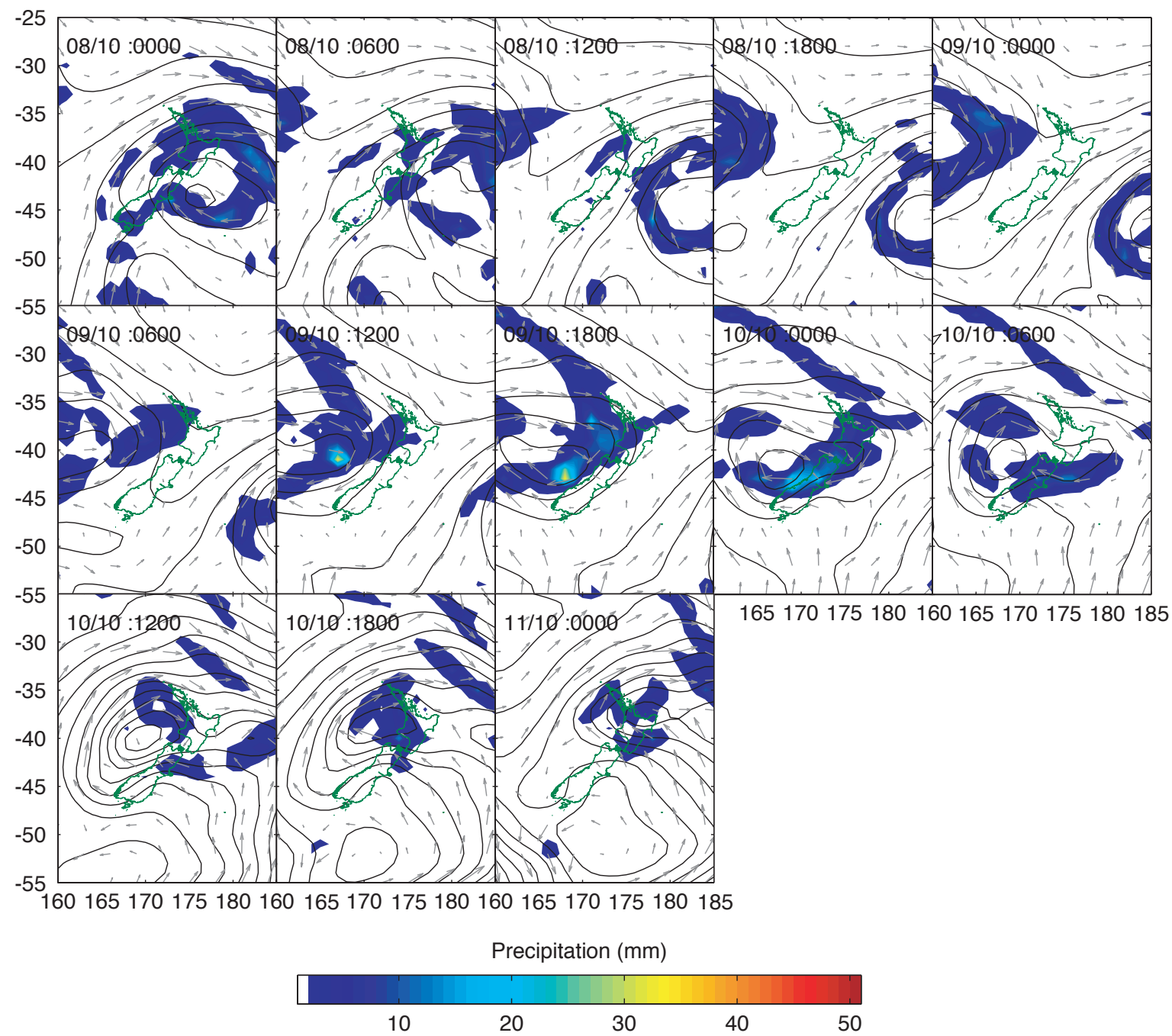

Figure 6.10 The six-hour lag precipitation forecasts for the New Zealand area during the October 2005 event according to the GFS model. The analysis files from these model runs were used to initialise the mesoscale simulations. This sequence shows the six-hour accumulation of precipitation from 0000 UTC 8 October to 0000 UTC 11 October.

\section{Event simulation}

The WRF model was used to simulate the precipitation in the Waikato River catchment during this event, from 0000 UTC on 5 October until 0000 UTC on 14 October. The most obvious difference between the GFS model and the WRF simulated six-hour precipitation accumulations (Figures 6.10 and 6.11), is that the WRF simulation produced significantly greater, and more widespread, precipitation. The simulation showed scattered precipitation in the area on 6 October, followed by 30 hours of continuous 


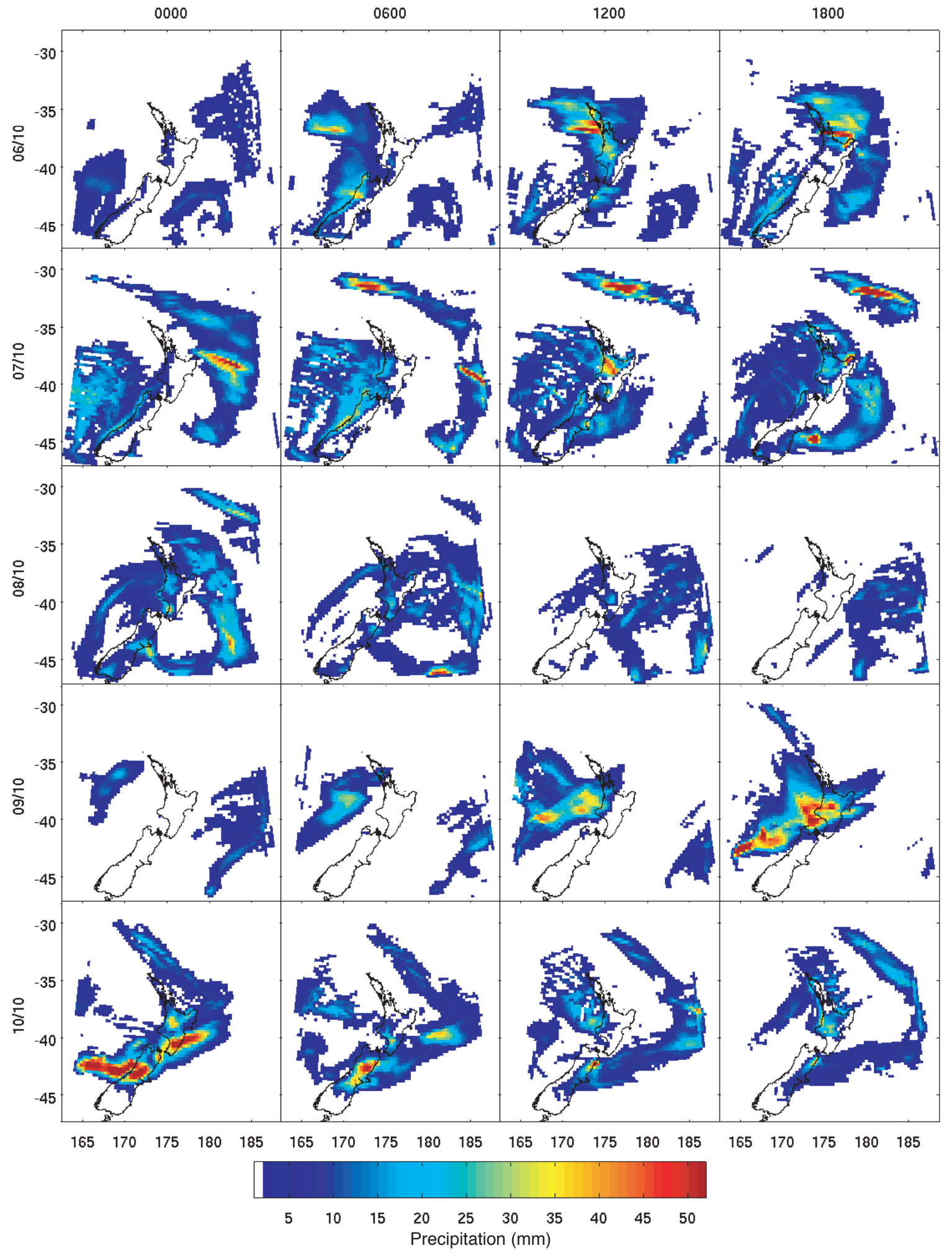

Figure 6.11 Six-hour precipitation accumulations from the WRF simulation on the 21-km grid for the October 2005 event. These maps are the combined convective and resolved precipitation in the outer domain. 
precipitation from 7 to 8 October. Another precipitation band approached from the northwest at 1200 UTC on 9 October, which led to heavy precipitation over the central North Island between 1200 UTC 9 October and 0000 UTC 10 October (coinciding with observed flooding in Gisborne and heavy precipitation seen in the daily time series). This was followed by more scattered precipitation until 0600 UTC 11 October.

Unlike the GFS model, the outer domain of the Control simulation contained the distinct precipitation periods observed in Figure 6.9. The total accumulation of simulated precipitation over the event (Figure 6.12) shows that the WRF model significantly improved the volume and distribution of precipitation compared to the GFS model. A simple visual comparison between the regional time series (Figure 6.13) shows that the timing of precipitation periods is consistent despite small discrepancies in magnitude.

Additional plots in Figures 6.12 and 6.13 show the precipitation generated in the NoTopo model run. As expected, there was less precipitation generated by the NoTopo simulation. Compared to the total quantity of observed precipitation, the Control run simulated $92 \%$ of the precipitation while the NoTopo run only predicted $58 \%$. Convective precipitation accounted for $64 \%$ of the generated precipitation in the NoTopo run but only $54 \%$ in the Control. Including the model topography had a greater influence on the resolved precipitation generated, but also significantly increased the total precipitation for this event in the Waikato River catchment.

Previous mesoscale simulations of extreme precipitation events showed a systematic under-prediction of the magnitude of the maxima (Zängl, 2007; Milbrant and Yau, 2001) of 10-15\%. In the simulation of Milbrant and Yau (2001) of the Saguenay (Canada) flood of 1996, the simulation was $10 \%$ lower than observed precipitation, and in the 48 hours of the event the total precipitation was down $15 \%$ when the topography was removed from the model. Although these cases are very different in location and mesoscale systems involved, it shows a similar result, the extreme precipitation is under-predicted and proper representation of topography increased the total volume of precipitation predicted. 

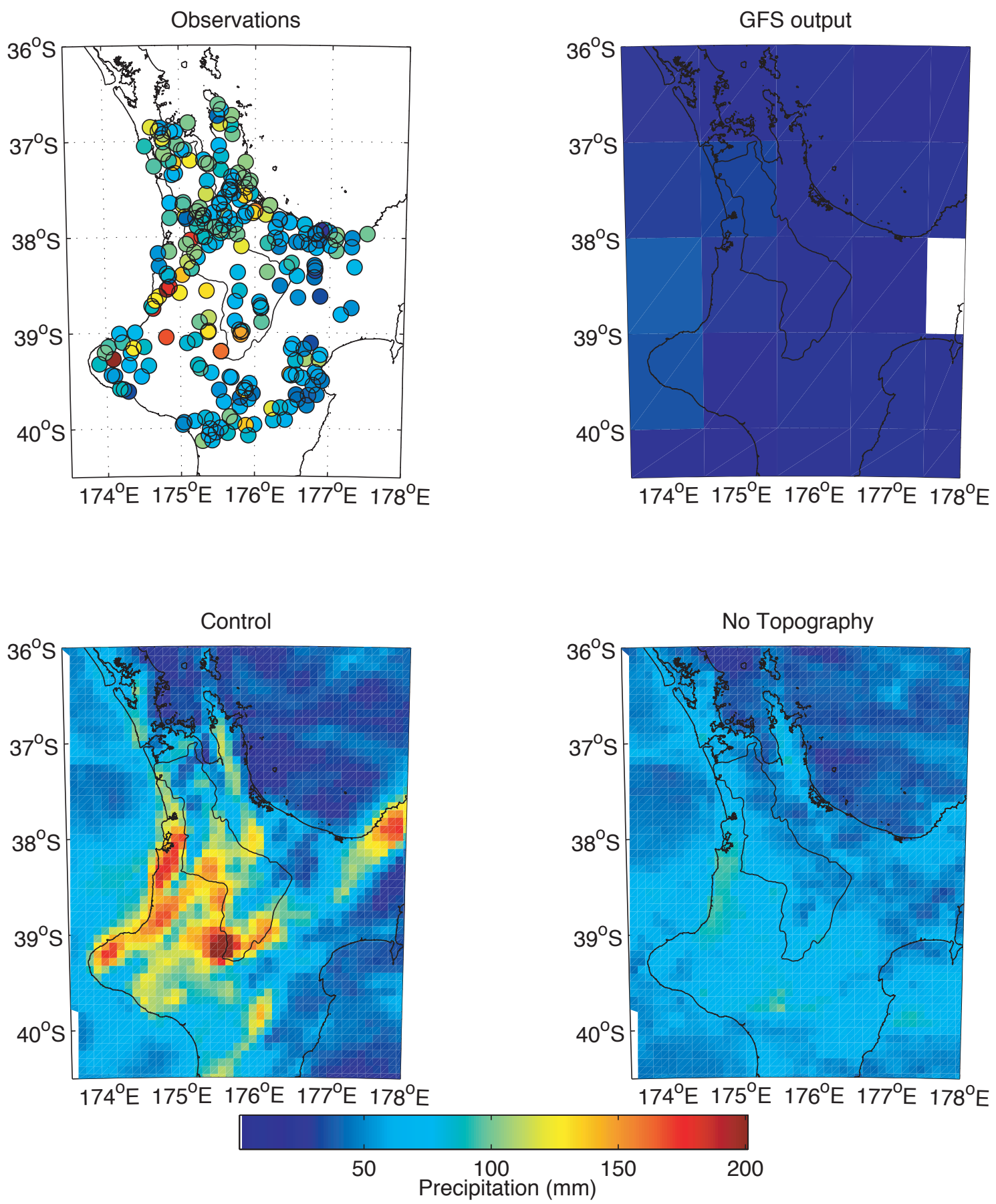

Figure 6.12 Accumulated precipitation over the October 2005 event. (a) Accumulated hourly precipitation from observations, (b) total accumulated precipitation from the GFS forecast in Figure 6.10, (c) total accumulated precipitation from the Control run of the WRF model, and (d) total accumulated from the NoTopo model run. 

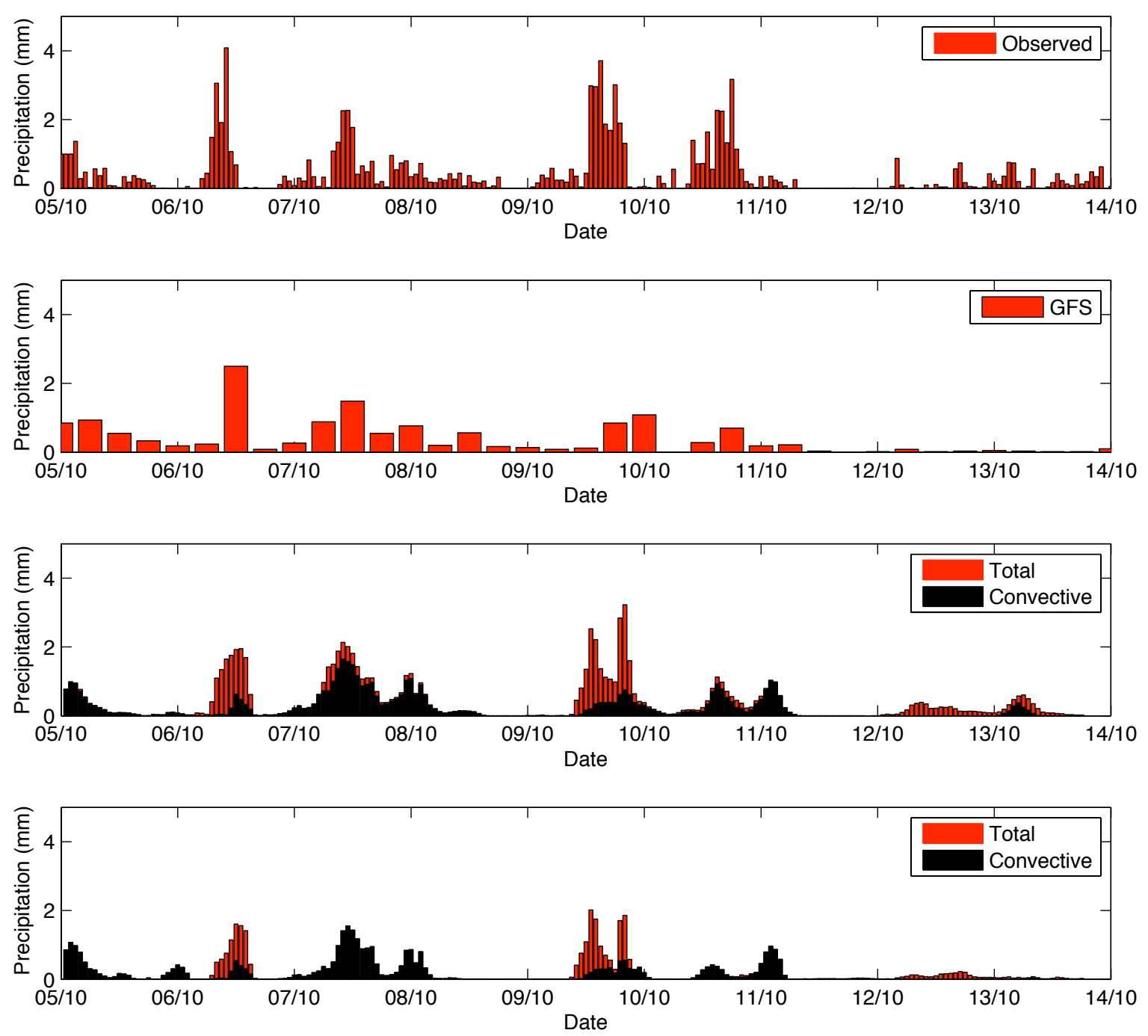

Figure 6.13 Area-averaged hourly resolved and convective precipitation time series for the nine- $\mathrm{km}$ grid of the October 2005 event for: (a) the observed regional hourly time series (b) the GFS 6 hour forecast (c) the Control model run and (d) the NoTopo model run.

\subsubsection{January 2006}

Torrential precipitation suddenly hit the Waikato Region on Wednesday 25 January $2006^{\dagger}$. On this single day the Waikato received more than $90 \%$ of its average January rainfall (MetService spokesperson, media release), sparking emergency call-outs and flooding throughout the region. However this heavy precipitation event was not unexpected. On 22 January the MetService issued the following warning:

\footnotetext{
†The Waikato Times, a daily Fairfax Media newspaper
} 
A deepening low is approaching New Zealand from the tropics. A strong or gale northeast airstream ahead of the low is expected to bring a burst of very heavy precipitation to northern and eastern areas from Northland to Gisborne with over $100 \mathrm{~mm}$ possible in a 12-hour period.

This was one of a number of warnings issued for the most of the North Island during the approach of this storm, although many of these warnings focused on the expected winds.

\section{Event observations}

Both the daily and five-day smoothed time series had peak precipitation values (40.0 $\mathrm{mm}$ and $17.6 \mathrm{~mm}$ respectively, Figure 6.14) above the 99th percentile for the January 2006 event. As mentioned earlier, the daily peak in this event was the largest recorded

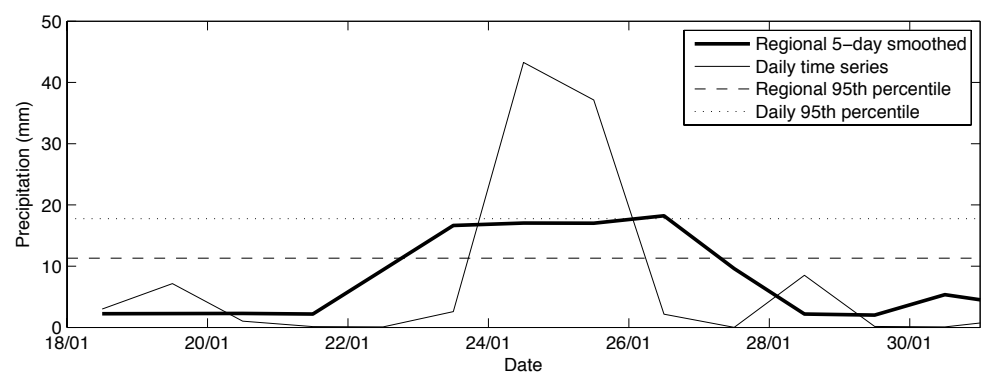

Figure 6.14 Daily precipitation time series for the January 2006 event. The thick solid line is the regional area-average smoothed time series. The thin solid line is the daily area-average time series. The dashed line shows the 95th percentile of the smoothed time series and the dotted line represents the 95th percentile of the daily time series.

during the two-year period for which the GFS data were available.

The warm front from the subtropical cyclone passed over the North Island at 0000 UTC (Figures 6.15 and 6.16) on 24 January but it was the arrival of the associated cold front approximately six hours later that caused the heaviest precipitation in the Waikato River catchment. The blocking high to the east of New Zealand restricted the southeastward movement of this cyclone. Early on 25 January the cold front associated with the Southern Ocean cyclone arrived in New Zealand. This second feature was 

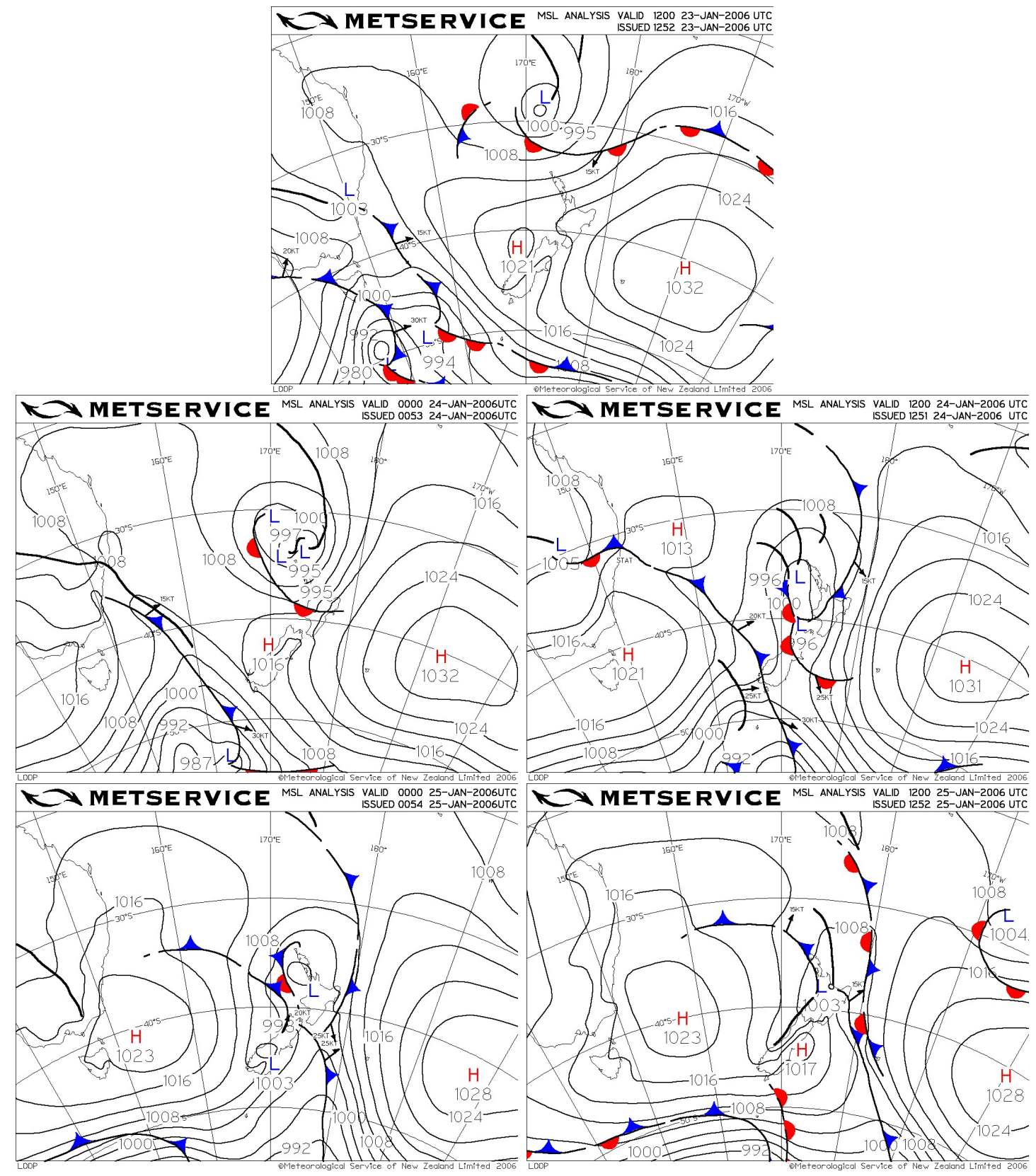

Figure 6.15 Selected MetService analysis maps for the January 2006 event. These maps are valid for: 1200 UTC 23 January; 0000 UTC 24 January; 1200 UTC 24 January; 0000 UTC 25 January; and 1200 UTC 25 January.

weaker but helped trap the warm tropical air producing convective precipitation on 25 January.

The hourly precipitation time series (Figure 6.17) shows the two distinct precipitation periods. The highest average peak was recorded late on 24 January (morning of 25 January local time). Precipitation was extensive throughout the Waikato River 


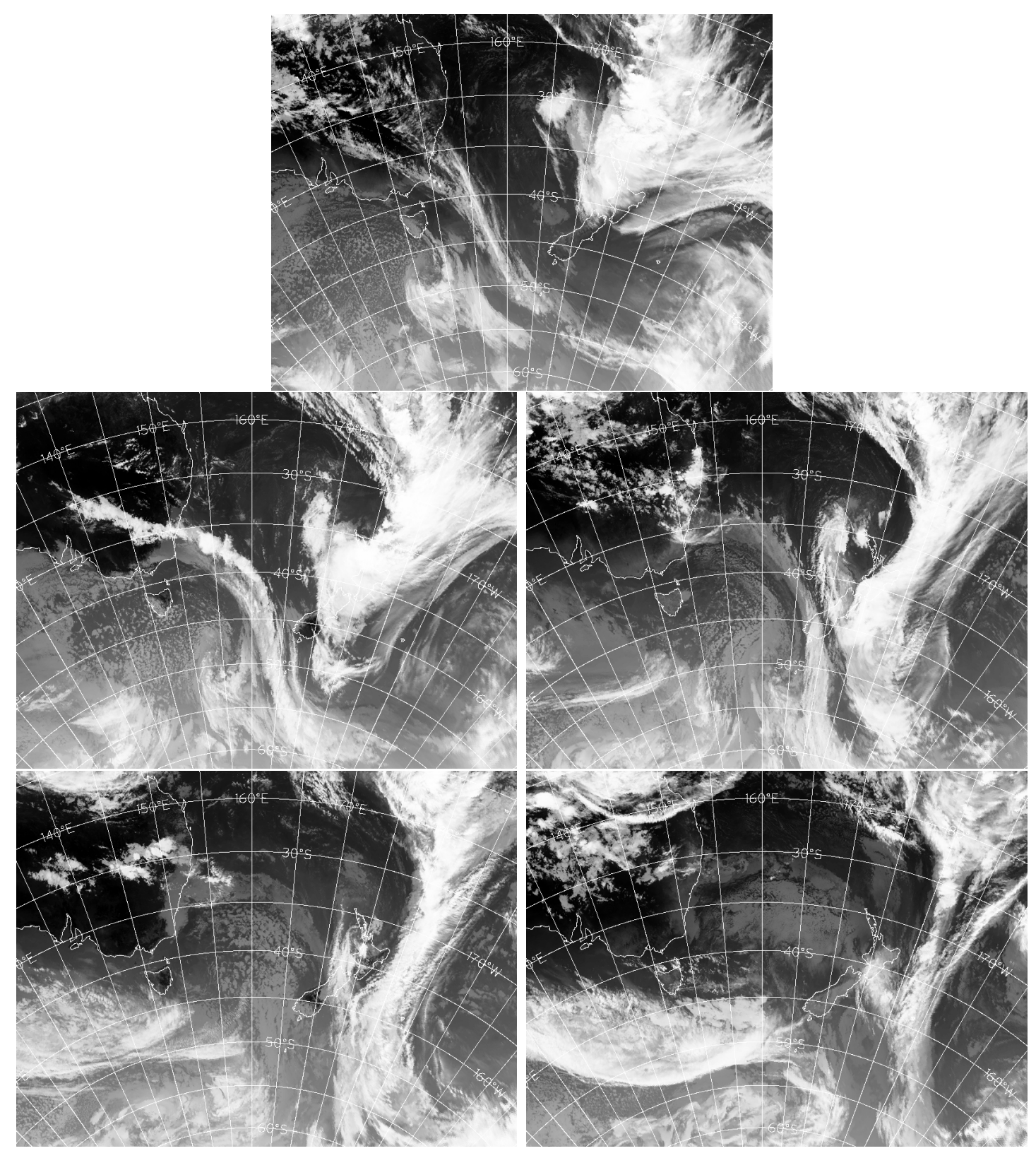

Figure 6.16 Selected satellite images for the January 2006 event. These maps are valid for: 1200 UTC 23 January; 0000 UTC 24 January; 1200 UTC 24 January; 0000 UTC 25 January; and 1200 UTC 25 January.

catchment but not simultaneous. Large isolated precipitation periods occurred on 25 January but these convective cells were unevenly distributed throughout the catchment. Light to moderate precipitation was spatially widespread over this event (Figure 6.20). However, larger volumes are seen in the Bay of Plenty and at sites that are at higher altitude throughout the North Island. 

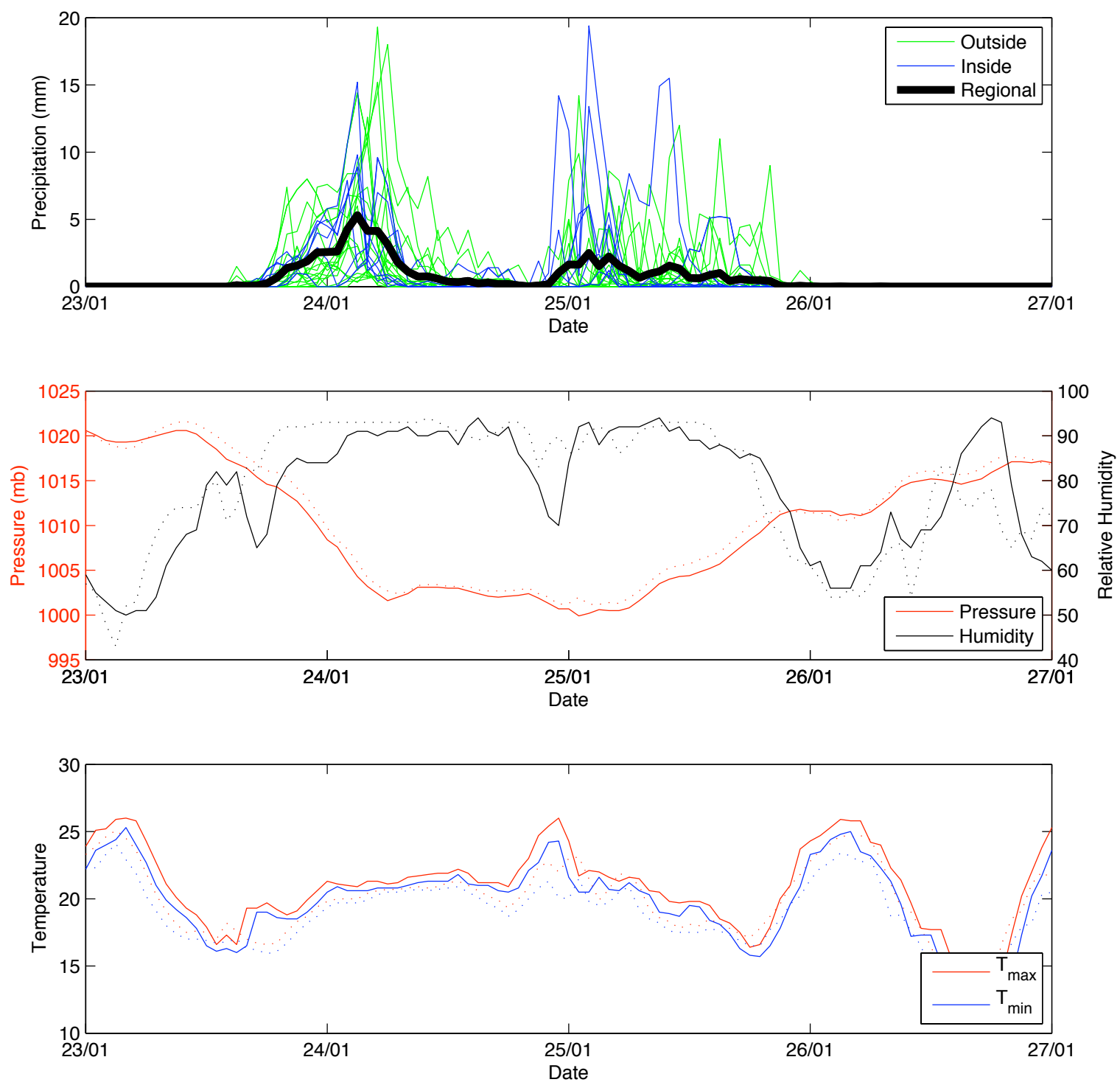

Figure 6.17 Hourly meteorological observations for the January 2006 event. (a) The hourly precipitation time series. Blue lines represent stations inside the river catchment boundary and the green lines are those outside. The thick line represents the average time series of the blue lines. Subplot (b) shows the average pressure (red) and relative humidity (black) throughout the event, and (c) shows the maximum (red) and minimum (blue) temperatures each hour throughout the event. Pressure is recorded at stations 2112 and 23899 (dashed line) and relative humidity and temperature are recorded at stations 23899 and 25162 (dashed line) (shown in Figure 6.5).

The pressure dropped by $15 \mathrm{mb}$ during the first period of precipitation (Figure $6.17 \mathrm{~b}$ ), then steadily increased after reaching a minimum of $\sim 1000 \mathrm{mb}$ at $0200 \mathrm{UTC}$ on 25 January. 


\section{Global model data}

The approach of the cyclone from the north is very obvious in the GFS model output (Figure 6.18). GFS predicted heavy precipitation covering most of the North Island

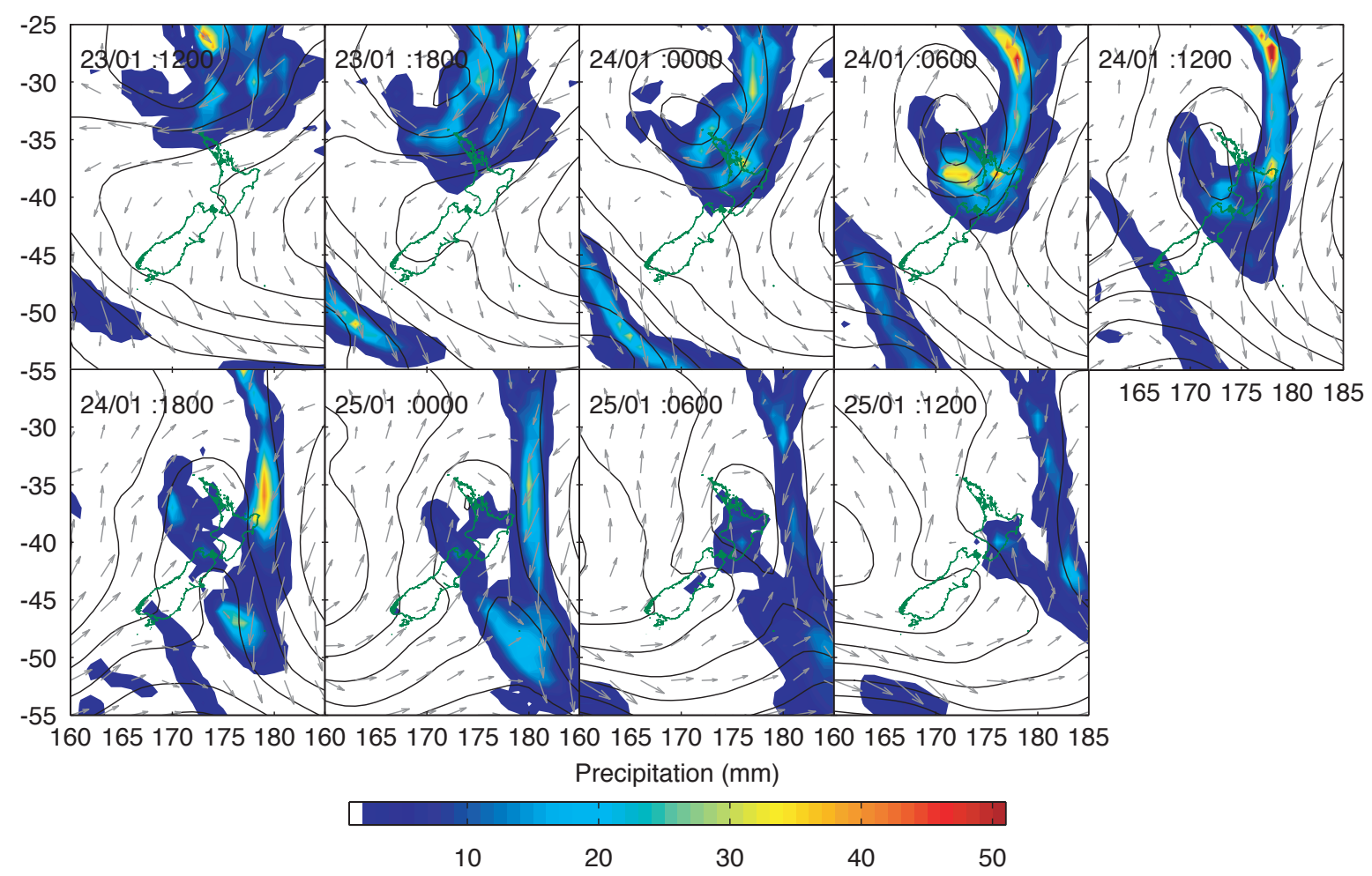

Figure 6.18 The six-hour lag precipitation forecasts for the New Zealand area during the January 2006 event according to the GFS model. The analysis files from these model runs were used to initialise the mesoscale simulations. This sequence shows the six-hour accumulation of precipitation from 1200 UTC 23 January to 1200 UTC 25 January.

between 0000 and 1800 UTC 24 January, and a distinct period of moderate precipitation again in the earlier parts of 25 January. The GFS model data are more consistent with observations in this event than in the October 2005 event.

\section{Event simulation}

The precipitation generated in the WRF simulation was from 1200 UTC on 23 January until 1200 UTC 27 January (Figure 6.19) is similar to that shown for the GFS forecast. However, as we would expect of a mesoscale simulation, there is more structure in 


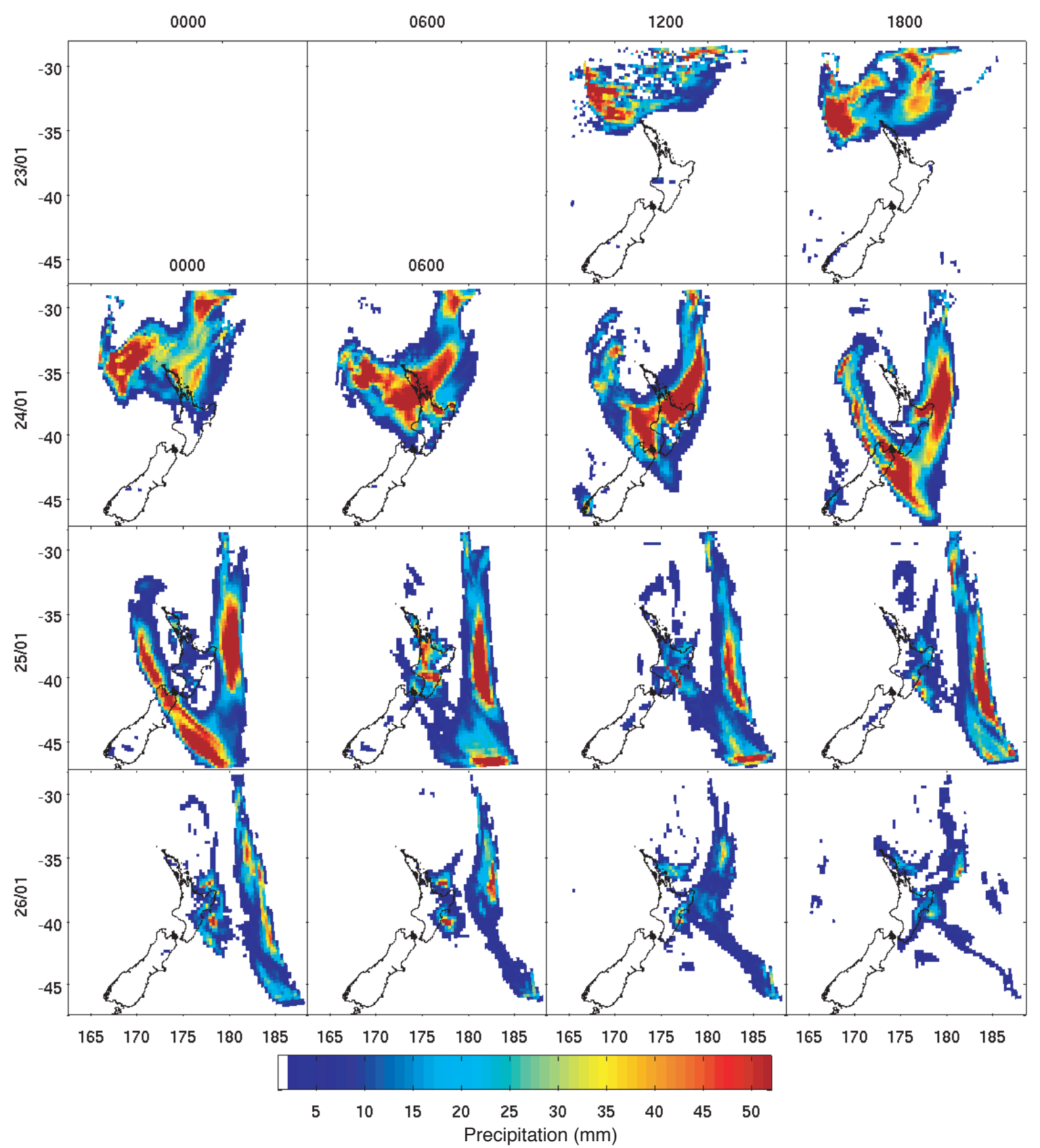

Figure 6.19 Six-hour precipitation accumulations from the WRF simulation for the January 2006 event. These maps are the combined convective and resolved precipitation in the outer domain.

the frontal bands providing more detailed spatial distribution and higher intensity precipitation predictions.

The four-day accumulation of total precipitation (Figure 6.20) shows that between 50 and $130 \mathrm{~mm}$ of precipitation fell within the Waikato River catchment. Outside of 

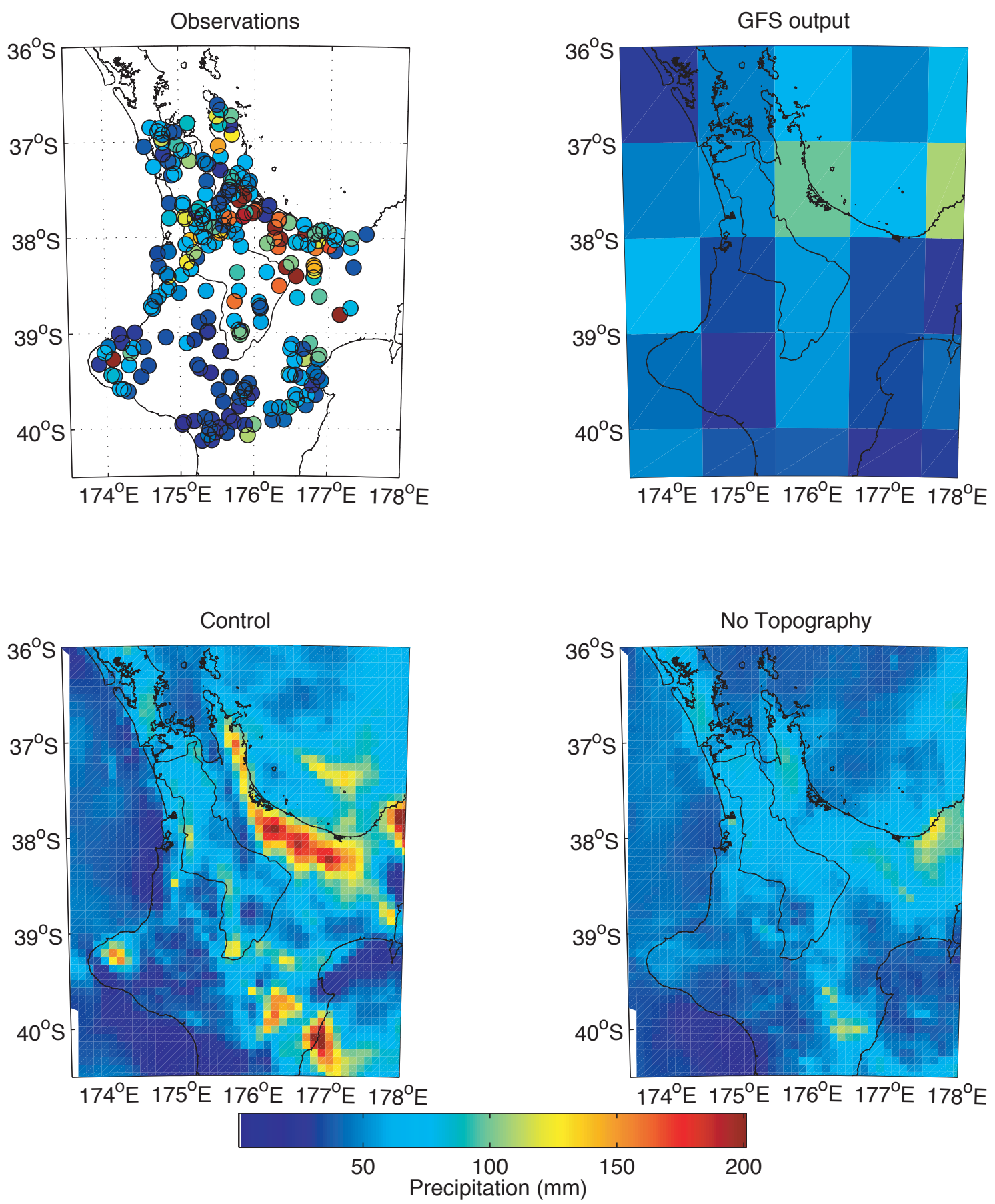

Figure 6.20 Accumulated precipitation over the January 2006 event. (a) Accumulated hourly precipitation, (b) total accumulated precipitation from the GFS output in Figure 6.18, (c) total accumulated precipitation from the Control run of the WRF model, and (d) total accumulated from the NoTopo model run. 
the catchment, precipitation totals for parts of the Bay of Plenty exceeded $200 \mathrm{~mm}$. The simulated precipitation is consistent with totals observed during the event. The hourly regional time series (Figure 6.21) comparison clearly shows the two distinct periods of precipitation. The peak of the first precipitation period, coinciding with
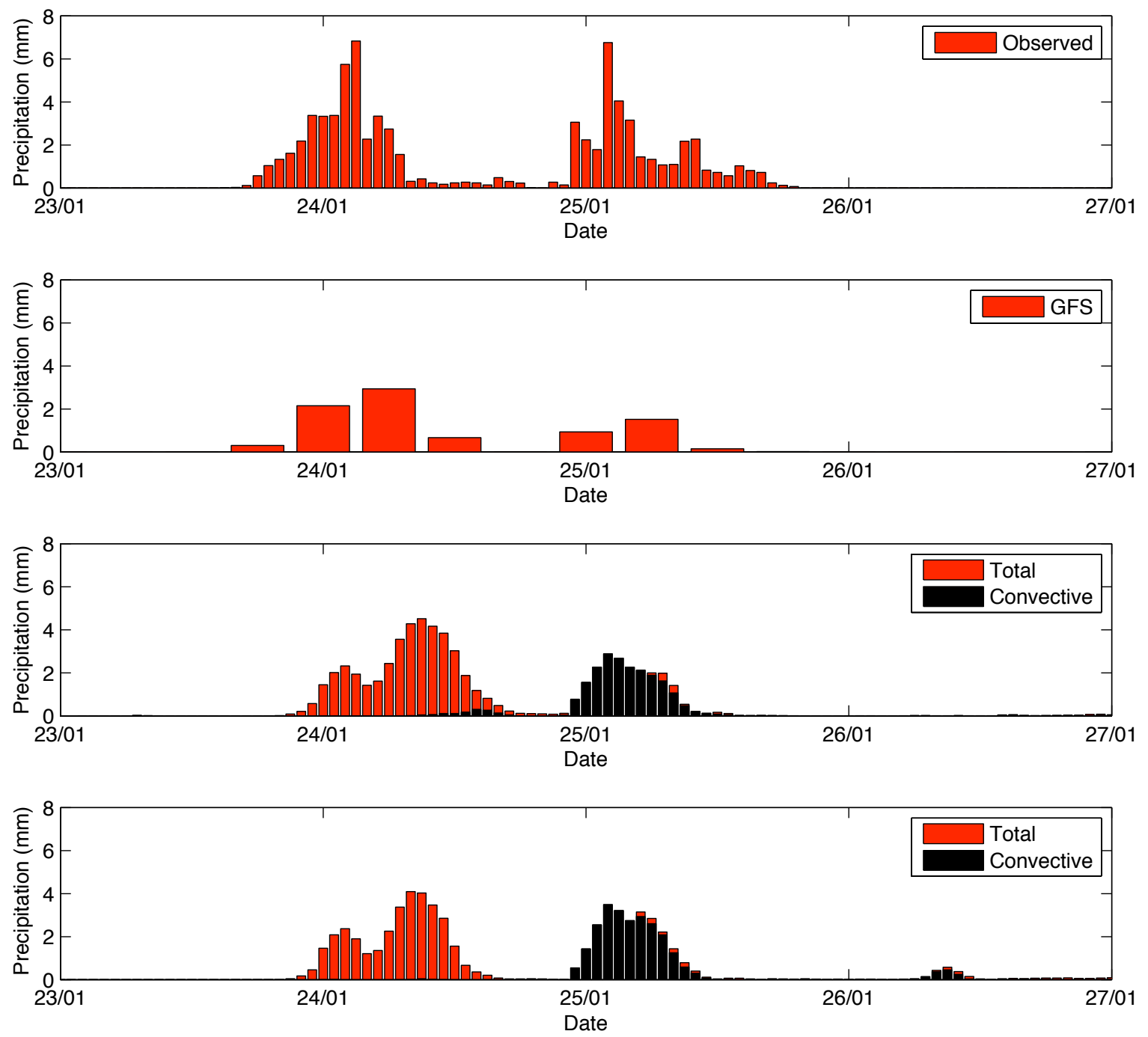

Figure 6.21 Area-averaged hourly resolved and convective precipitation time series for the nine-km grid of the January 2006 event for: (a) the observed regional hourly time series (b) the GFS 6 hour forecast (c) the Control model run and (d) the NoTopo model run.

the subtropical cyclones cold front, occurred approximately four hours ahead of the simulated fronts. However, as the total accumulated precipitation was consistent with observations this indicates that the timing of the front was the main error. The GFS output (Figure 6.18) also timed this precipitation later than it occurred so the timing 
error (of only four hours) was inherited from the global model.

From the NoTopo simulation in Figure 6.20 it can be seen that orographic enhancement played an important part in the distribution of precipitation, especially in the areas when extremely high precipitation totals were simulated in the Control run. The hourly time series (Figure 6.21) shows that the NoTopo model run produced no convective precipitation during the frontal period of the event, but produced additional convective precipitation in the convective phase, including an additional convective precipitation event at 1000 UTC 26 January that was not observed. Without the orographic enhancement during the frontal phase of the event the moisture levels in the atmosphere were higher, allowing for additional convective precipitation to be generated when convection was initiated. The resolved precipitation in both cases had similar timing and relative sizes; however, the total volumes produced were smaller in the NoTopo run, especially in the frontal period. Convection accounted for $33 \%$ of the simulated precipitation (or $40 \%$ in the NoTopo run) and only $83 \%$ of the total precipitation was simulated in the Control and $80 \%$ in NoTopo. However, the distribution of precipitation was significantly altered in the NoTopo simulation.

\subsubsection{October 2006}

After three days of warm unstable air producing thunderstorms, a mid-latitude cyclone formed in the Tasman Sea in late September 2006. This cyclone was blocked from moving eastward by a high to the east of New Zealand. This event consisted of thunderstorms, heavy downpours, localised flooding and even a tornado. The event caused a number of houses in Auckland and Wellington to be evacuated due to landslips. But there was no damage reported in the Waikato River catchment and the regional time series did not exceed the 95th percentile despite heavy rain warnings being issued by the MetService. 


\section{Event observations}

Precipitation peaked at $24.8 \mathrm{~mm}$ on 1 October 2006, above the 97 th percentile of the regional daily time series (Figure 6.22); a similar amount of precipitation was observed

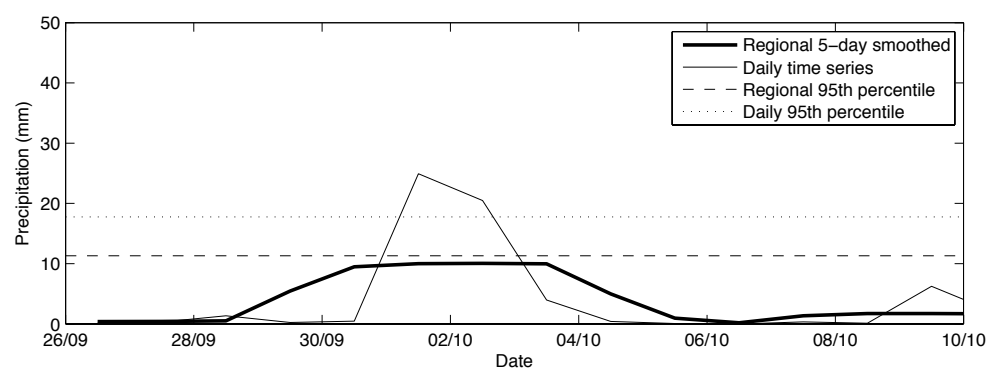

Figure 6.22 Daily precipitation time series for the October 2006 event. The thick solid line is the regional area-average smoothed time series. The thin solid line is the daily area-average time series. The dashed line shows the 95th percentile of the smoothed time series and the dotted line represents the 95th percentile of the daily time series.

again the following day. However, the smoothed time series peaked at $9.4 \mathrm{~mm}$, which is only in the 91st percentile and therefore did not meet the definition of an event applied in this research.

The slow-moving cyclone formed in the Tasman Sea on 30 September (Figures 6.23 and 6.24) and became very complex in the unstable environment west of New Zealand. The mid-latitude cyclone took three days to pass over the landmass of New Zealand.

The hourly precipitation time series (Figure 6.25) showed that heavy precipitation was localised. Yet it was raining somewhere in the North Island almost constantly from 0000 UTC 1 October to 0000 UTC 4 October. The heaviest precipitation occurred during the first two of these days, with pressure decreasing by $\sim 13 \mathrm{mb}$ during this time. As the pressure began to increase after 0000 UTC 3 October, the relative humidity dropped and the diurnal signal in temperature returned.

The spatial distribution of precipitation (Figure 6.28) is more varied for this event than for the previous case studies and total volumes are smaller. Most of the heavy precipitation cells occurred in the Auckland area, but high levels of precipitation also occurred along the west coast and in the Bay of Plenty. 


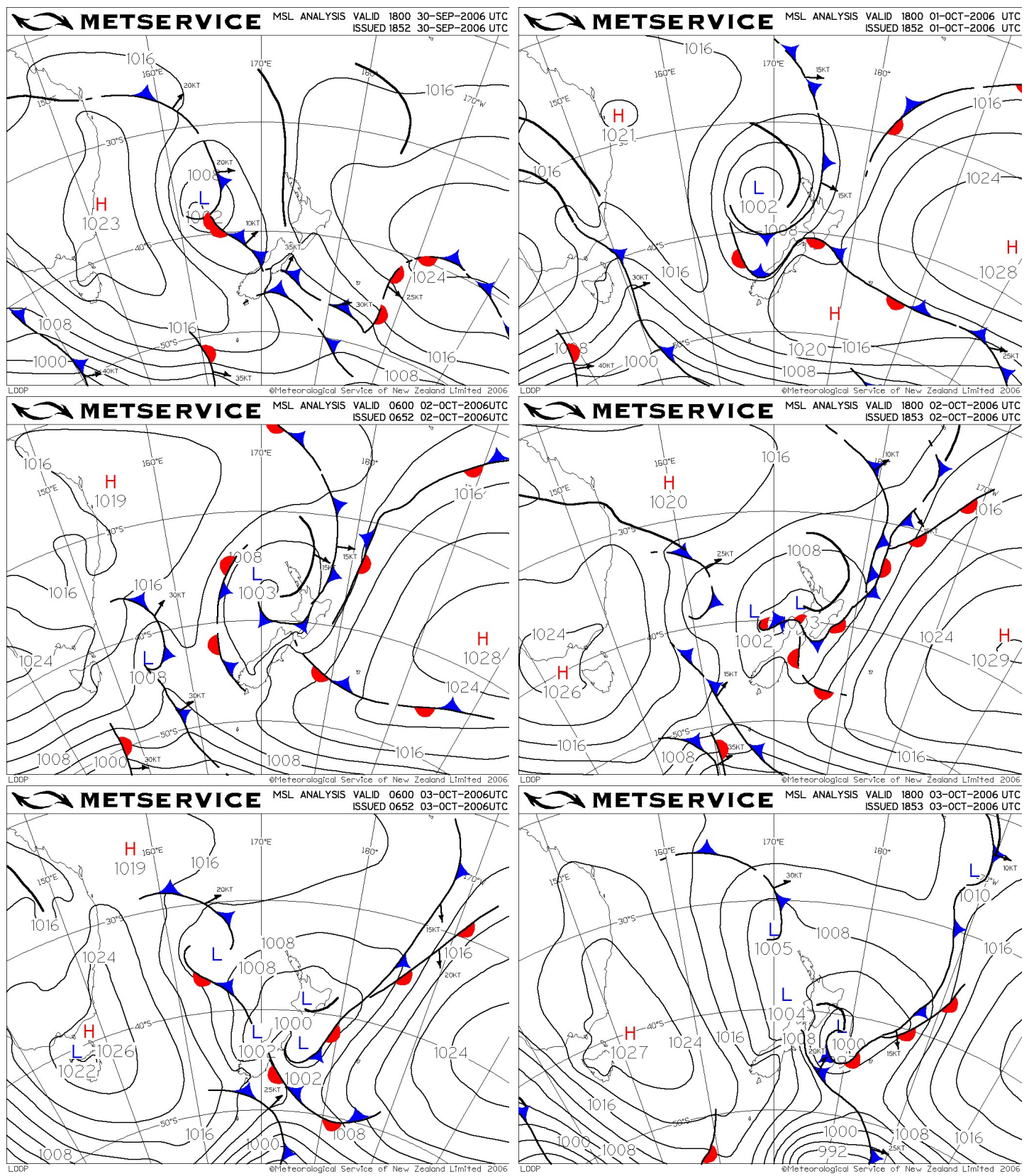

Figure 6.23 Selected MetService analysis maps for the October 2006 event. These maps are valid for: 1800 UTC 30 September; 1800 UTC 1 October; 0600 UTC 2 October; 1800 UTC 2 October; 0600 UTC 3 October; and 1800 UTC 3 October.

\section{Global model data}

The GFS model output (Figure 6.26) shows a large low sitting west of New Zealand from 1800 UTC 30 September to 1800 UTC 2 October, when the central minimum filled and traversed across the North Island. These forecast charts also show the high to the east of New Zealand that initially prevented the cyclone from moving westward. 

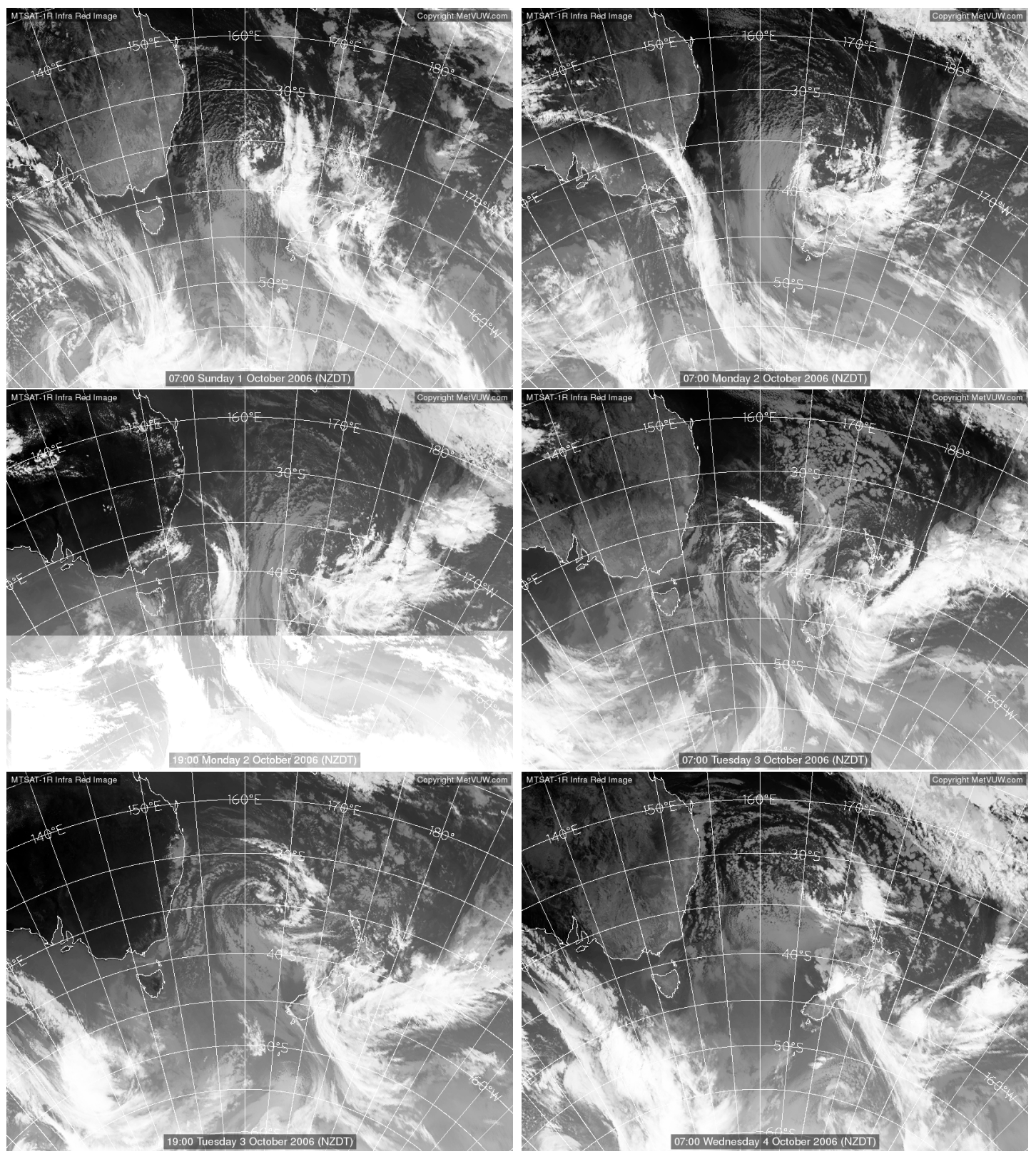

Figure 6.24 Selected satellite images for the October 2006 event. These maps are valid for: 1800 UTC 30 September; 1800 UTC 1 October; 0600 UTC 2 October; 1800 UTC 2 October; 0600 UTC 3 October; and 1800 UTC 3 October.

However, despite the similarities in the pressure fields of the GFS output and the analysis maps there was little precipitation predicted near the central North Island in the GFS output. 

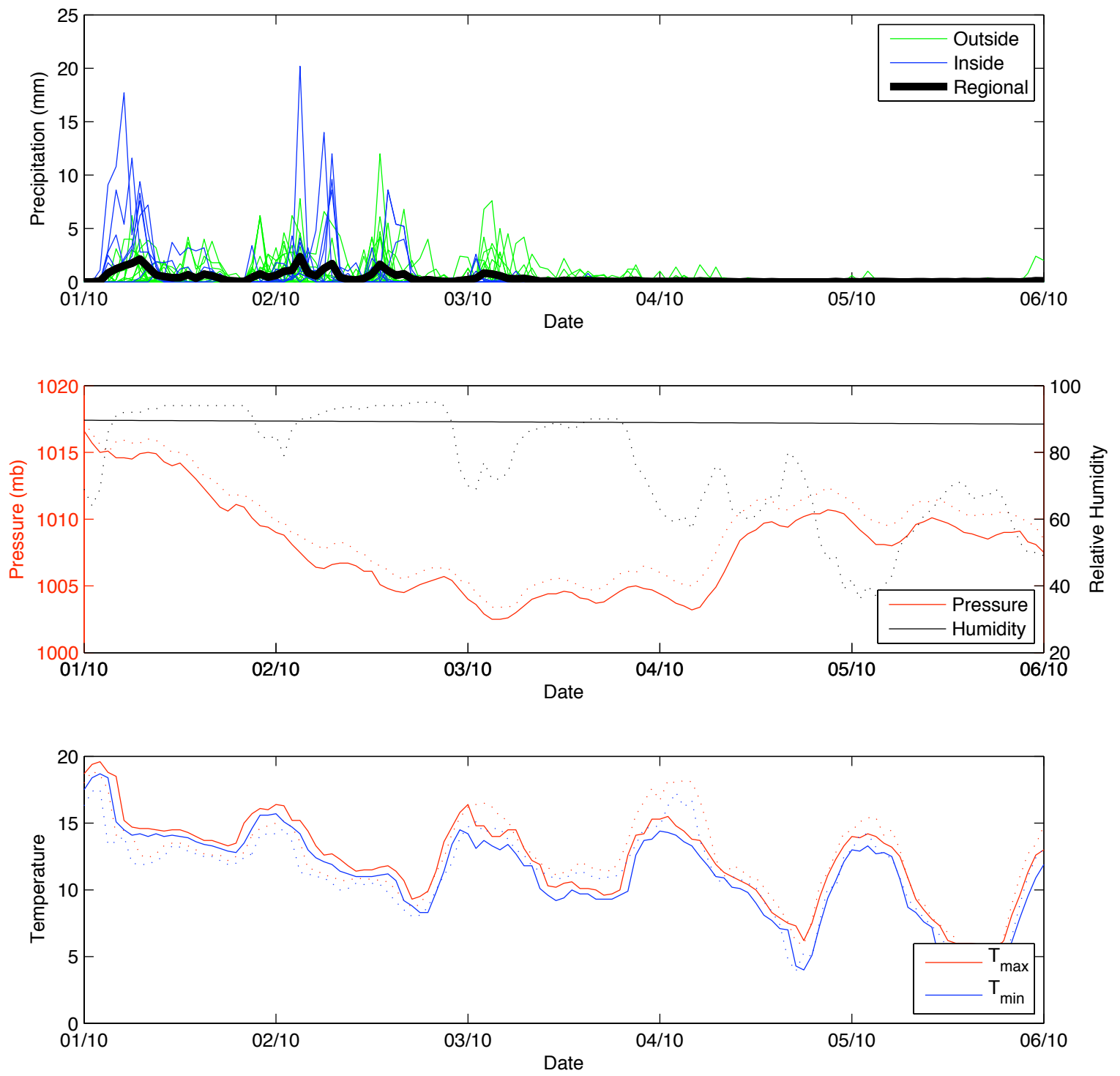

Figure 6.25 Hourly meteorological observations for the October 2006 event. (a) The hourly precipitation time series. Blue lines represent stations inside the river catchment boundary and the green lines are those outside. The thick line represents the average time series of the blue lines. Subplot (b) shows the average pressure (red) and relative humidity (black) throughout the event, and (c) shows the maximum (red) and minimum (blue) temperatures each hour throughout the event. Pressure is recorded at stations 2112 and 23899 (dashed line) and relative humidity and temperature are recorded at stations 23899 and 25162 (dashed line) shown in Figure 6.5. Note that station 23899 failed to record the relative humidity during this event. 

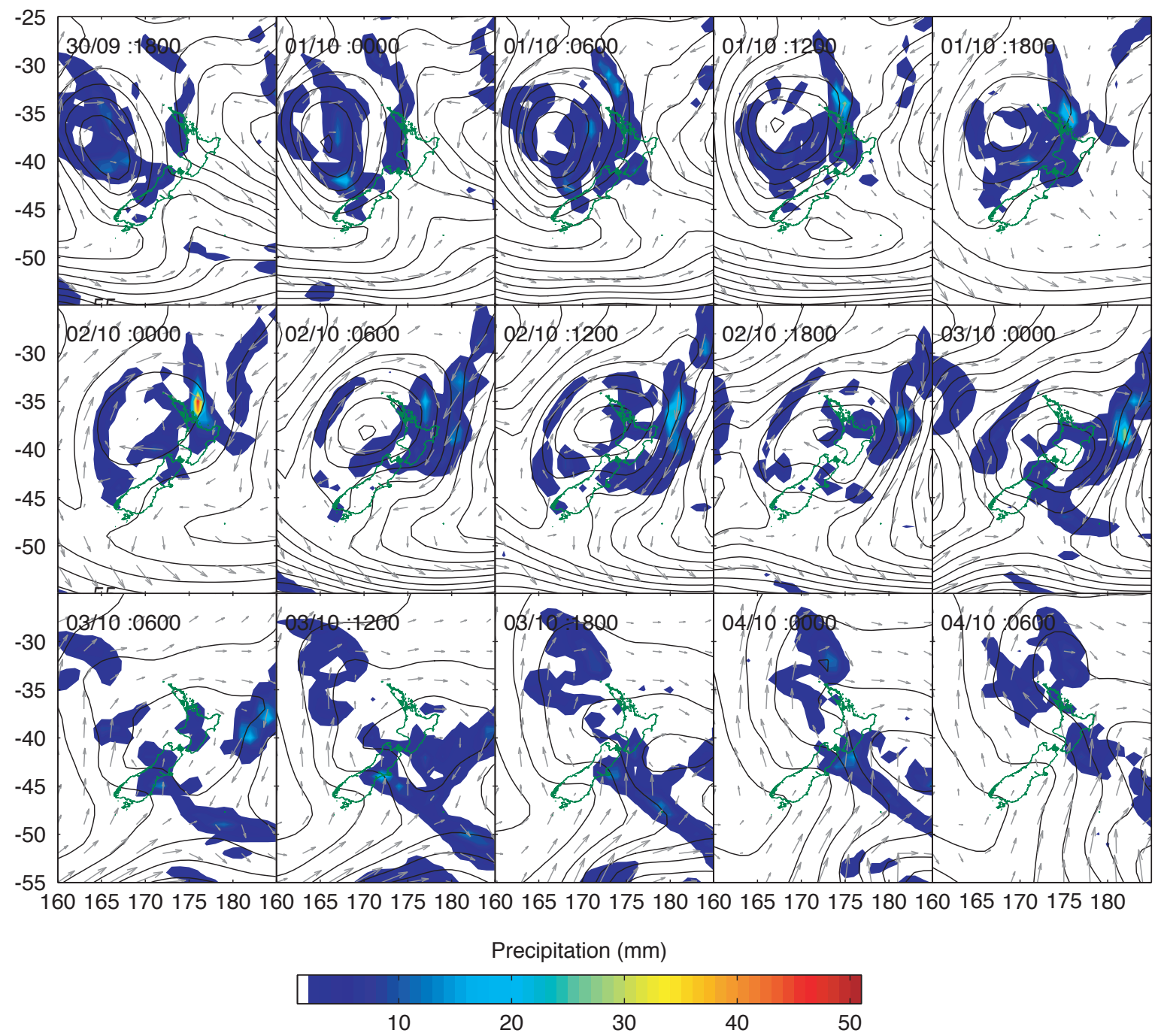

Figure 6.26 The six-hour lag precipitation forecasts for the New Zealand area during the October 2006 event according to the GFS model. The analysis files from these model runs were used to initialise the mesoscale simulations. This sequence shows the six-hour accumulation of precipitation from 1800 UTC 30 September to 0600 UTC 4 October.

\section{Event simulation}

The control simulation (Figure 6.27) generated scattered precipitation throughout the region from the start of the model run at 0000 UTC 1 October until 1800 UTC 4 October. There is heavier precipitation occurring at 0600 UTC 1 October and 0600 UTC 2 October which both coincide with observations. However, the hourly observation time series had almost no precipitation occurring after 0000 UTC 3 October, whereas the model was still predicting moderate precipitation throughout the area. 


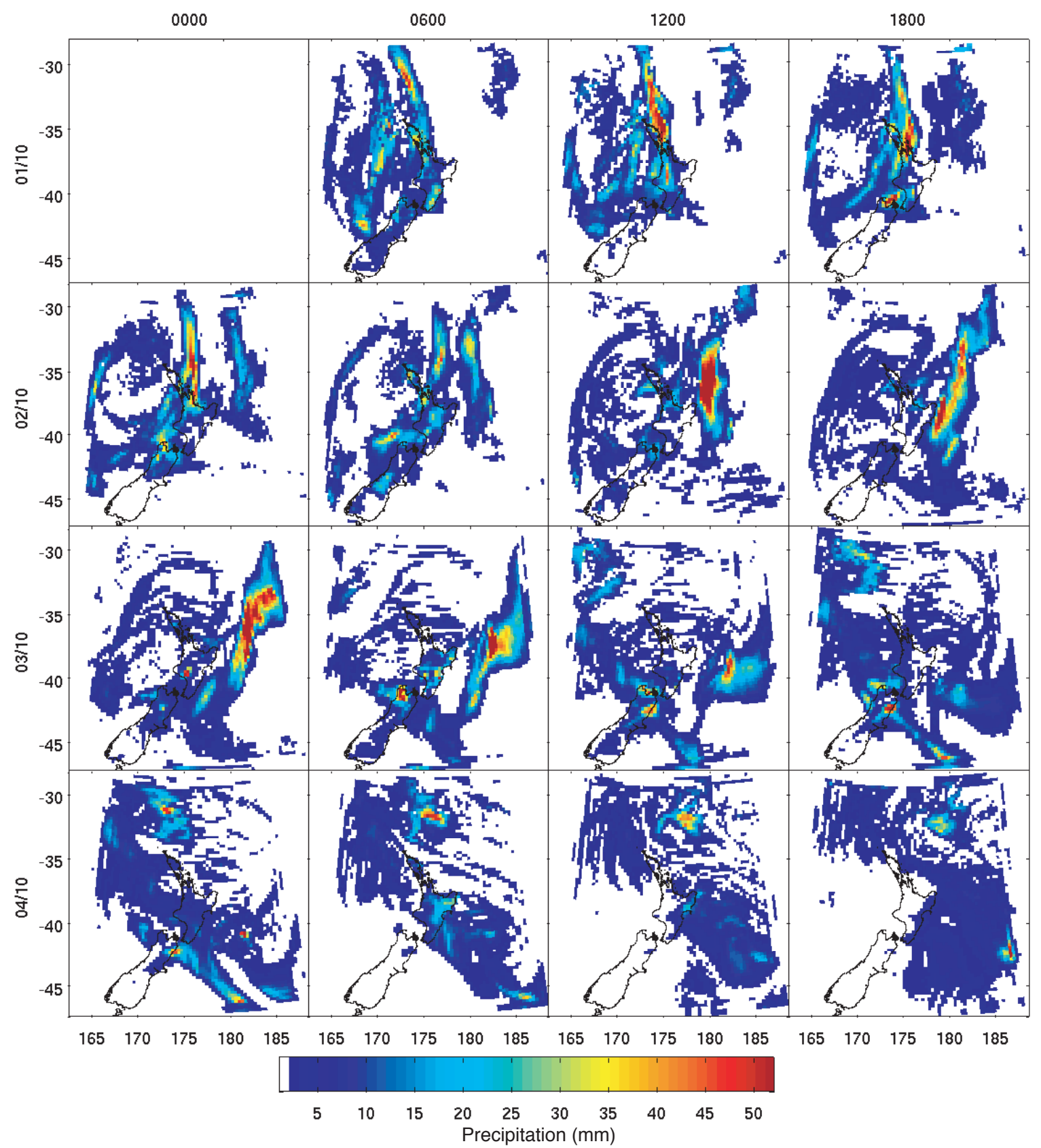

Figure 6.27 Six-hour precipitation accumulations from the WRF simulation for the October 2006 event. These maps are the combined convective and resolved precipitation in the outer domain.

When the total precipitation is accumulated over the event (Figure 6.28) it can be seen that observed precipitation near Auckland exceeded that which was modelled, while in most other locations the model has over-predicted the precipitation. The model has produced higher levels of precipitation on the western side of the topographic 

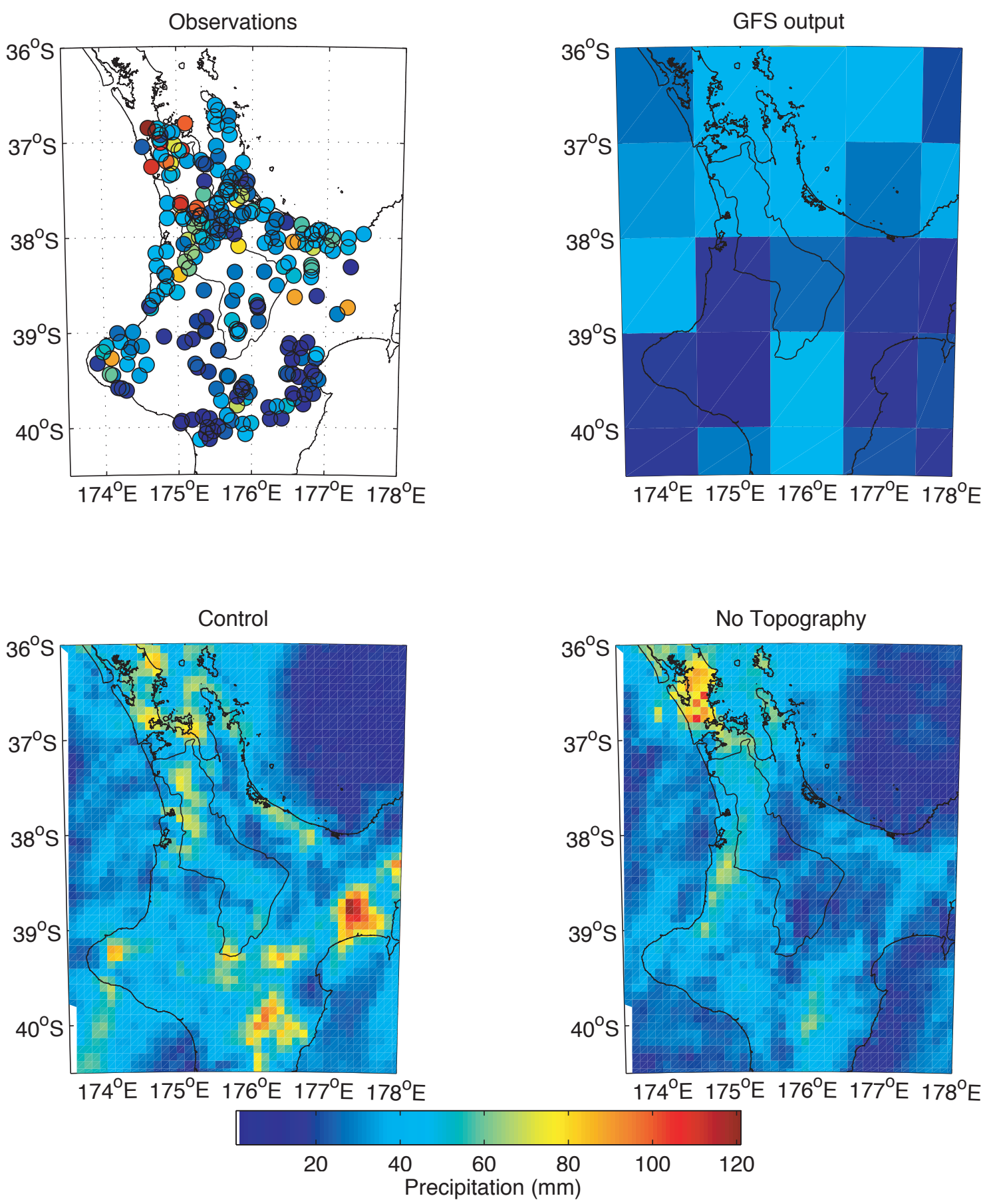

Figure 6.28 Accumulated precipitation over the October 2006 event. (a) Accumulated hourly precipitation, (b) total accumulated precipitation from the GFS output in Figure 6.26, (c) total accumulated precipitation from the Control run of the WRF model, and (d) total accumulated from the NoTopo model run. 
features that is consistent with the direction of the moving frontal band. The hourly time series (Figure 6.29) shows four periods of convective precipitation that coincided with local afternoon/evening. However, the simulated precipitation is less intense and
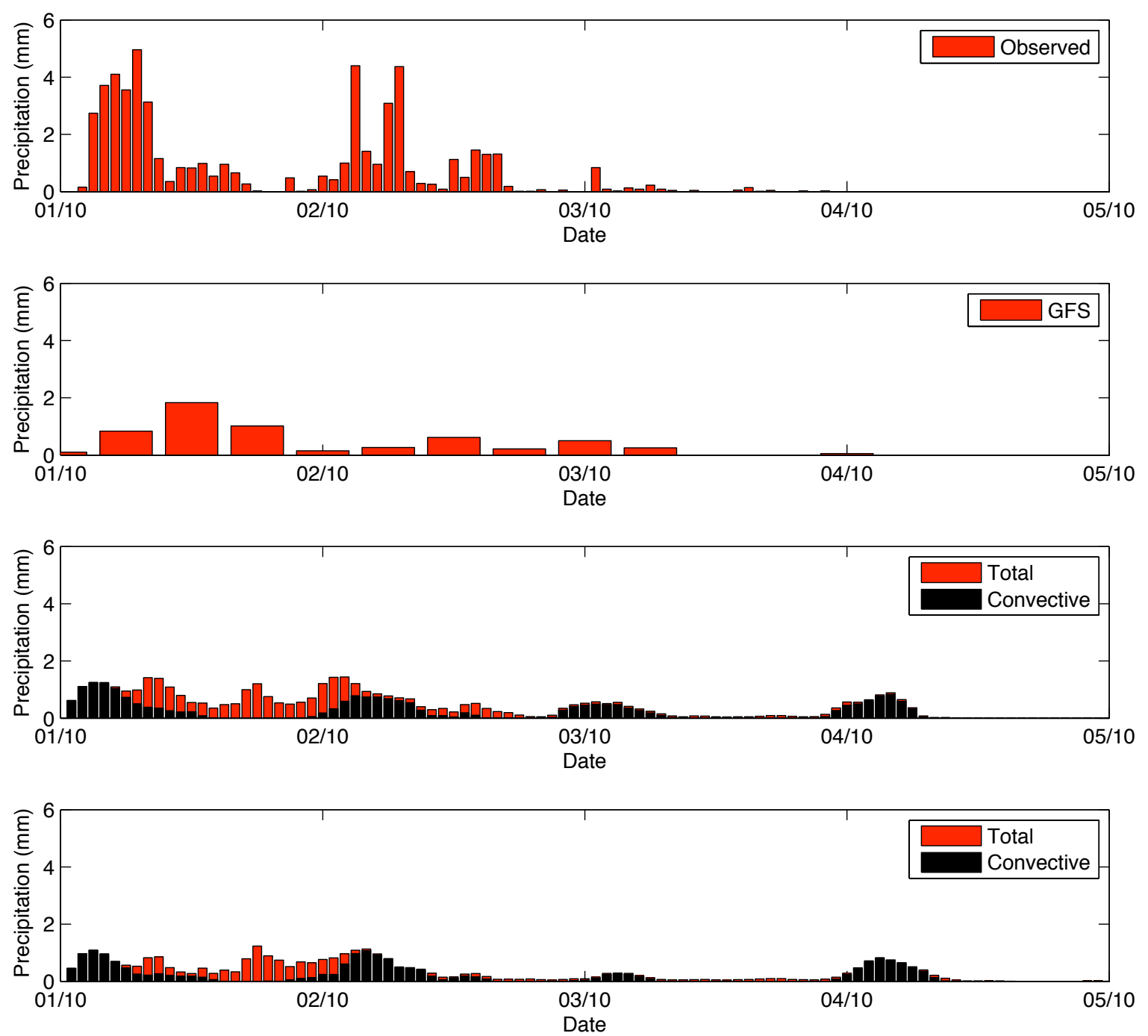

Figure 6.29 Area-averaged hourly resolved and convective precipitation time series for the nine-km grid of the October 2006 event for: (a) the observed regional hourly time series (b) the GFS 6 hour forecast (c) the Control model run and (d) the NoTopo model run.

more persistent than the observed totals.

The NoTopo simulation produced a smoother precipitation distribution over the majority of the North Island although it significantly increased the total precipitation predicted in Auckland. The NoTopo simulation only generated slightly less precipitation than the Control, but did reduce the total prediction from $78 \%$ to $60 \%$ of the 
observed value. In this event, convective processes accounted for $52 \%$ (or $59 \%$ in the NoTopo run) of the precipitation and it is the lack of physical constraint on convective precipitation (and caution when issuing a public forecast) that is likely to have led to the precipitation warnings issued for the Waikato by operational forecasters prior to this event.

\subsection{Parameter sensitivity}

As mentioned earlier, there are numerous options available in the WRF model that affect how precipitation is generated. This section discusses the precipitation generated in ten separate simulations of the January 2006 event to show the effects of some of these options. The multiple runs show the sensitivity of the precipitation generation to the model input and options, thereby showing the limitations of predicting precipitation with the mesoscale model. This event was chosen because it contained both of the major influences on simulated precipitation, i.e. it contained both frontal and convective precipitation, and the distribution of precipitation was dependent on the topographical resolution of the simulation; thereby testing multiple abilities of the model in a single event.

The Control simulation is as described in section 6.1.1. In each additional simulation one model option was altered. Their unique setting in the coming text identifies the simulations and the differences are shown in Table 6.2. The model runs are split into four categories, representing variations in topography, microphysics, cumulus parameterisation, and resolution respectively. For each of the four categories, the hourly precipitation time series are compared at six observation stations, the rms errors for each of the stations are plotted, and plots of the accumulated precipitation generated in the inner domain during the respective frontal and convective periods are presented. 


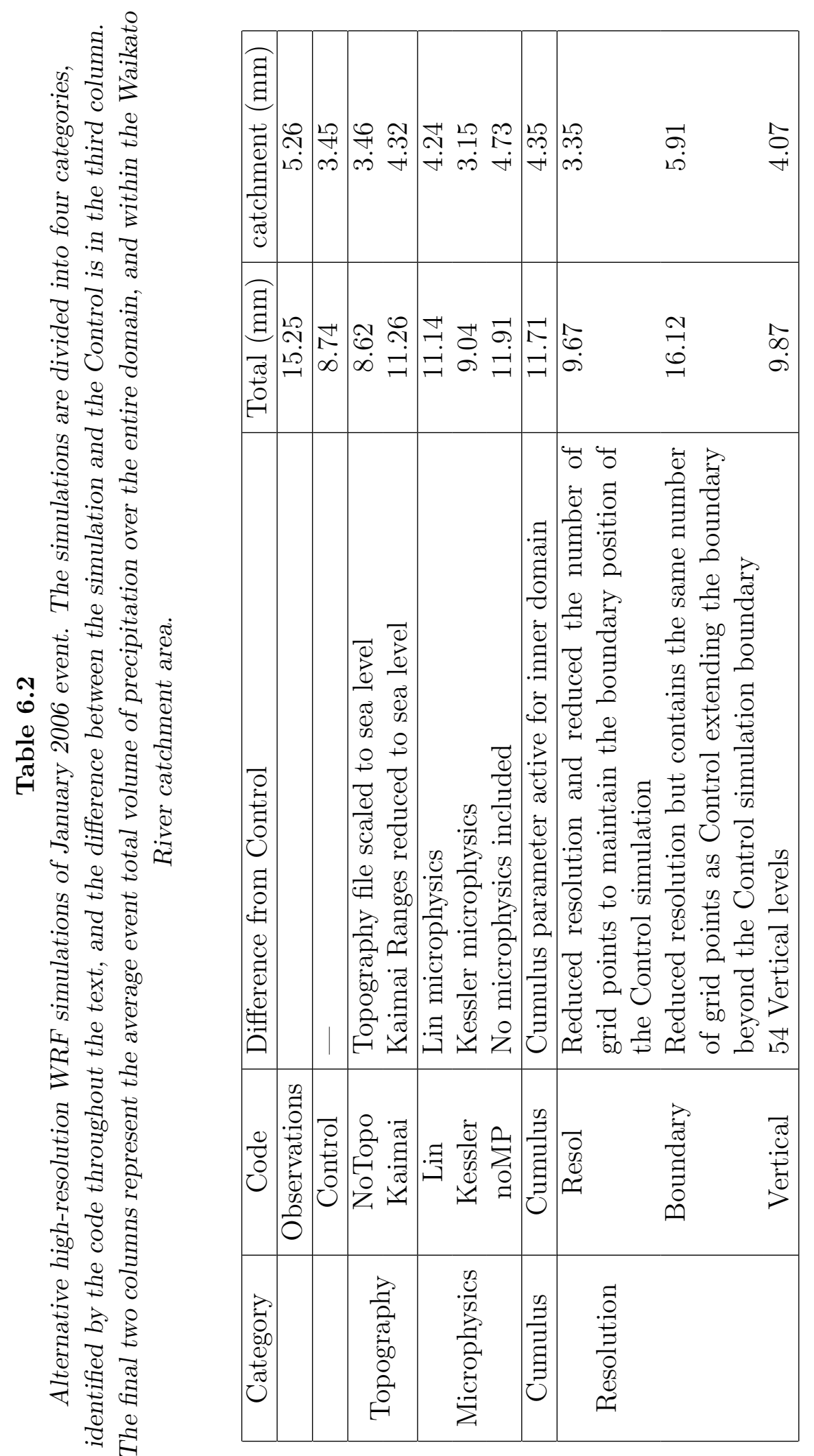




\subsubsection{Topography}

The largest precipitation errors are expected when complex topography is poorly represented (as in the global model) as topography is one of the most important parameters for determining the distribution of precipitation (Buzzi et al., 1998, Italy). The topographic effect is much more likely to be influenced by the type and direction of the approaching system. Therefore a NoTopo simulation was conducted for each of the case studies as shown above. Scaling all topography to sea level in the NoTopo simulations reduced the total precipitation generated in each case study (precipitation accumulations are shown in Table 6.3), and showed that the magnitude of the topographic effect

Table 6.3

The total average amount of precipitation within the catchment area in the middle domain of the model for both the Control and NoTopo simulations, with the resolved and convective precipitation described separately in $\mathrm{mm} /$ area/event.

\begin{tabular}{|c|c|c|c|c|c|c|c|}
\hline \multirow{2}{*}{ Event } & \multirow{2}{*}{ Observed } & \multicolumn{3}{|c|}{ Control } & \multicolumn{3}{c|}{ NoTopo } \\
& & Resv & Conv & total & Resv & Conv & total \\
\hline October 2005 & 96.10 & 40.88 & 47.73 & 88.61 & 19.81 & 35.95 & 55.76 \\
\hline January 2006 & 78.53 & 43.68 & 21.56 & 65.24 & 37.59 & 25.35 & 62.92 \\
\hline October 2006 & 55.00 & 20.49 & 22.28 & 42.77 & 13.64 & 19.49 & 33.12 \\
\hline
\end{tabular}

was dependent on the case. The dependence of this effect on the weather system was also noted by Zängl (2007), and Colle (2003) noted that terrain had a minor role in precipitation during Hurricane Floyd and radiative processes and latent heating had much more effect.

This discussion of the topographic effects also includes the simulation referred to as 'Kaimai', where just a parallelogram (see Figure 6.5) of topographic data covering the Coromandel and Kaimai Range was scaled to sea level. This simulation should show the extent of the sheltering effect by this mountain range during this event. Both Milbrant and Yau (2001) and Colle and Mass (2000) artificially scaled sections of topography to investigate the role of orographic forcing in high-resolution simulations. Specifically Colle and Mass (2000) removed the coastal range from a simulation to show the barrier 
effects on precipitation downwind over the Cascades (in the United States of America), and in their case, the quantity of low level ice was reduced. However, the reduction in precipitation seen in the case studies here are not related to ice as there was no low level ice in these events.

Hourly regional time series comparisons are shown in each of the case studies (Figures 6.13, 6.21, and 6.29). Additionally the hourly time series for the January 2006 event from the Control, NoTopo and Kaimai simulations are shown in Figure 6.30 for each of the six stations marked in Figure 6.5. The distribution of precipitation

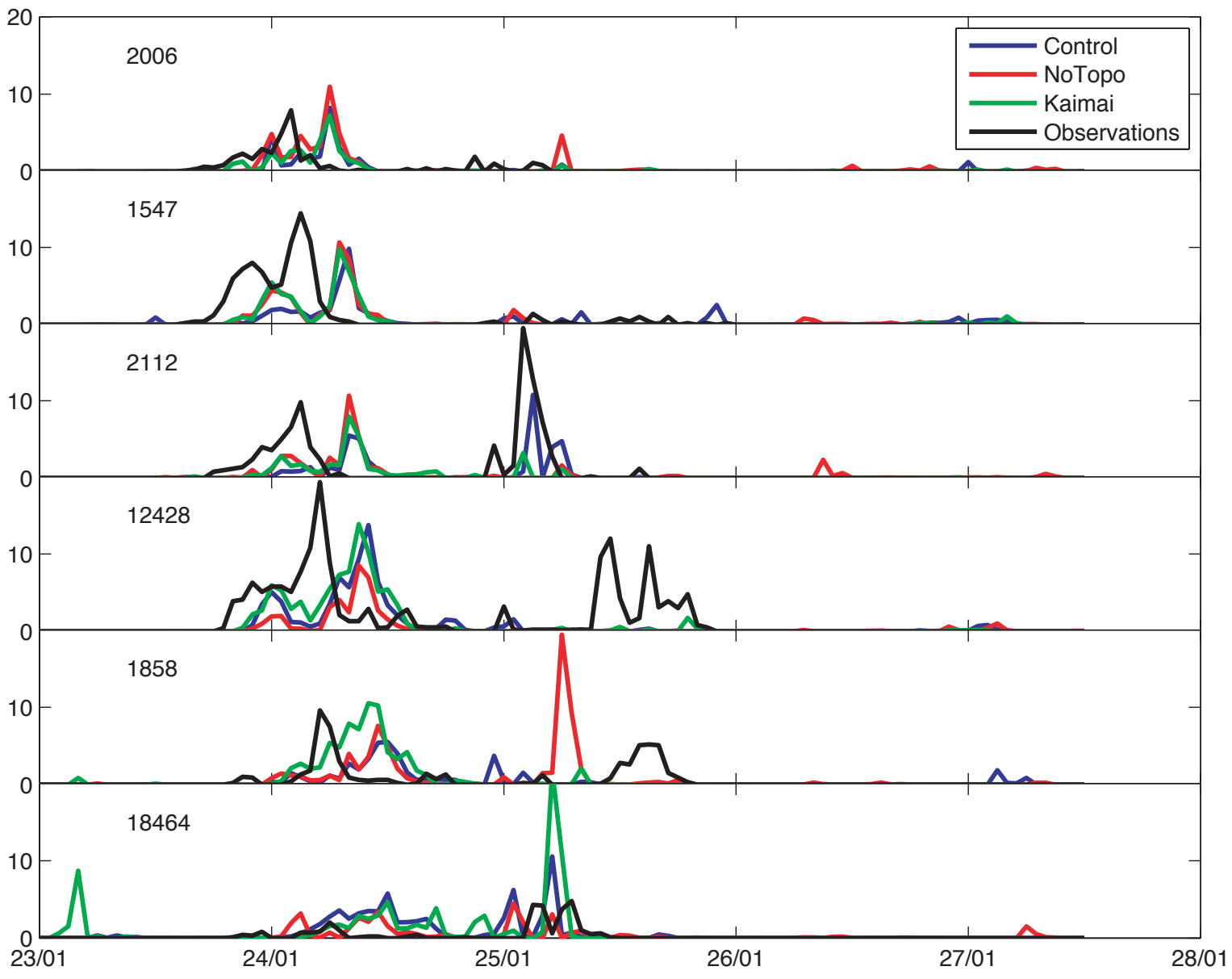

Figure 6.30 Hourly precipitation observations and simulated time series comparison from each of the topographic simulations at selected rain gauge locations identified in Figure 6.5.

during each of the precipitation periods are also mapped in Figures 6.32 and 6.33 . The simulated frontal precipitation peak was later than observed. The simulations 
under-predicted the precipitation at stations in the east in this frontal period but overprediction occurred on the west coast in the NoTopo run. The further northeast the site the larger the precipitation peak during the frontal period and the greater the difference between the observation and the simulation (ignoring the timing difference).

In the convective period of the event, there was more precipitation observed in the centre of the North Island (and therefore near the head of the Waikato River). Light to moderate precipitation was simulated at most stations, however, they all underpredicted the precipitation at station 2112. As expected, the Control run had the best agreement with observations for most stations. However, no simulation matched the precipitation observed at the two stations near the head of the Waikato River on 25 January. This could be due to the convective parameterisation placing the precipitation in an incorrect location as there were cells of precipitation throughout the region at the time.

The Kaimai simulation also generated additional precipitation in the western side of the domain during the frontal period, suggesting that this range sheltered the Waikato River catchment during this period. During the convective period the Kaimai simulation generated very little precipitation at most locations with the notable exception of station 18464 on Mt Ruapehu, where a large unobserved precipitation peak was simulated.

The rmse was calculated from a comparison of the hourly simulated time series and the observed time series at each of the 25 stations in the central North Island (Figure 6.31). In this point comparison of the simulation (even on the 3 -km resolution) it can be seen that most of the differences are smaller when topography is included in the model run (as expected). As the time series over any event is short a bootstrap confidence of the rmse value was calculated and the size of the dots in Figure 6.31 (and similar plots) are proportional to the confidence, i.e. larger dots indicate a less certain value. The confidence of the rmse at each station was similar between the simulations in this section. When the topography was excluded, the rms errors increased in the 

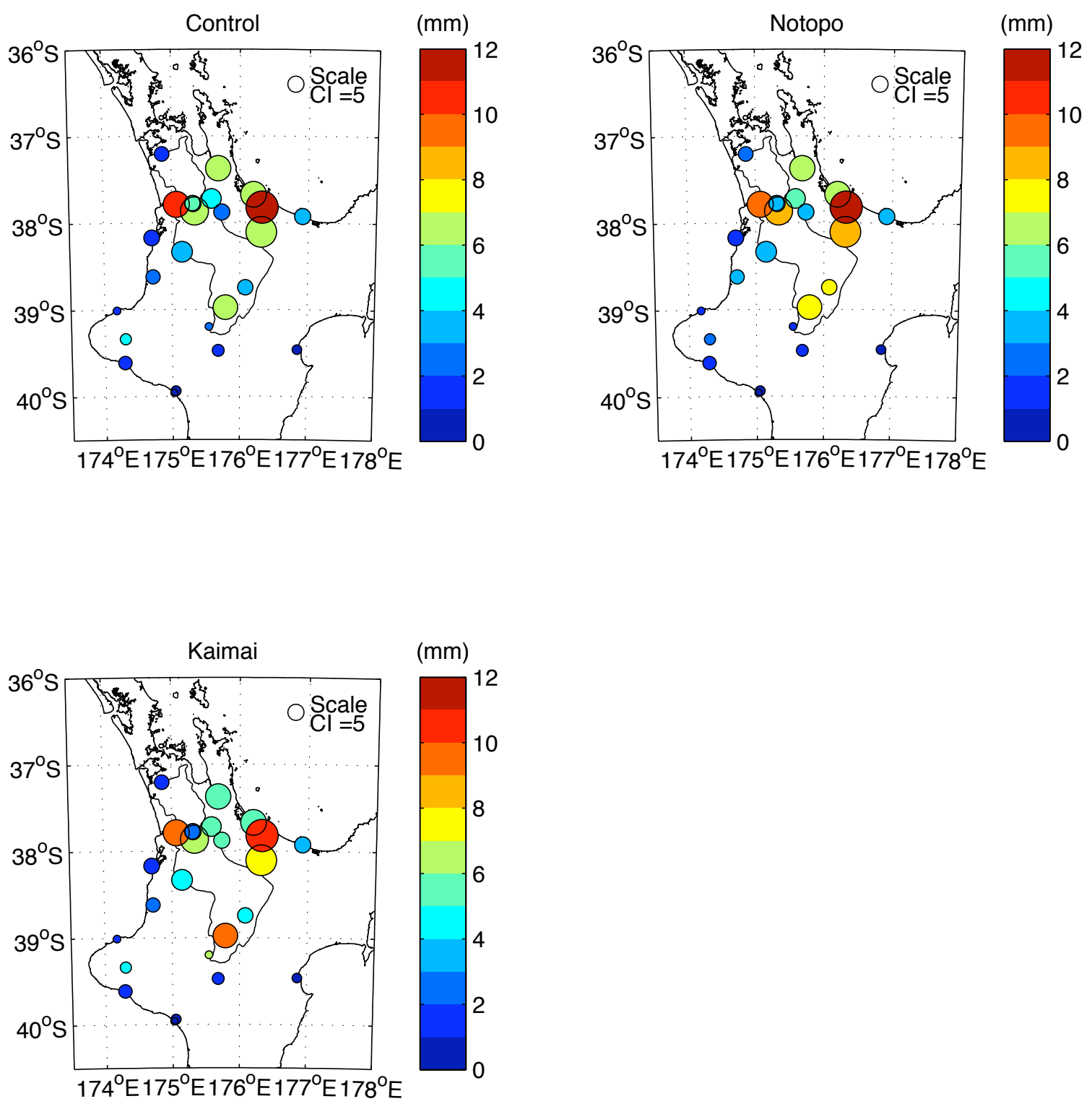

Figure 6.31 The rmse for individual hourly precipitation over the event for each of the topographic simulations. The size of the dots is proportional to the 0.05 confidence bootstrap interval.

central and western parts of the domain, but the rms errors were not altered in the south where precipitation totals were lower.

Figure 6.32 shows the total resolved and convective precipitation accumulated over the six-hour period ending at 0900 UTC 24 January in the inner domain, and Figure 6.33 is the 24-hour accumulation ending at 1200 UTC 25 January, representing the passage of the front and the convective periods respectively. The inner domain shows 

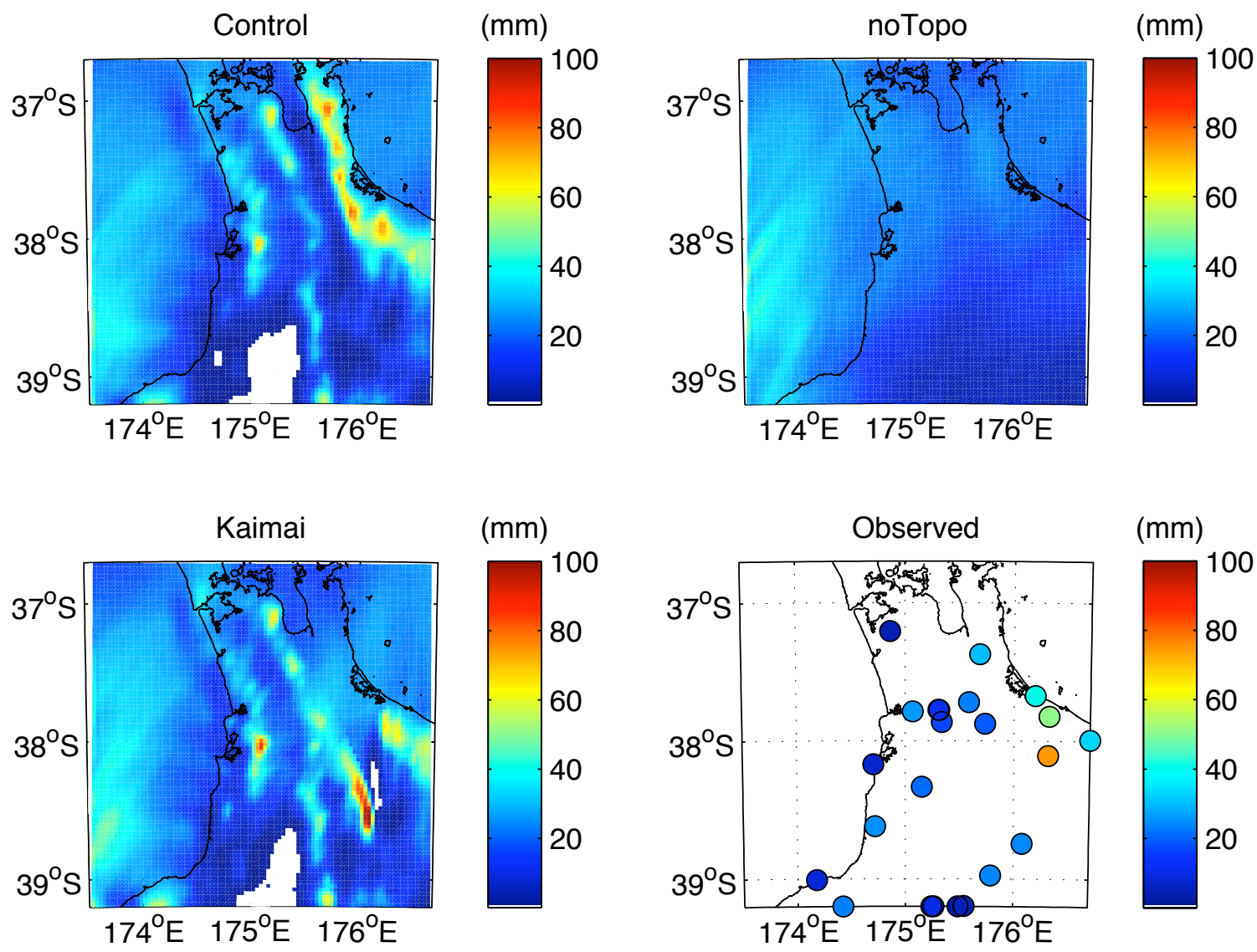

Figure 6.32 Six-hour precipitation accumulation during the frontal period for the topographical simulations. This shows the total precipitation generated in the six hours prior to 0900 UTC 24 January 2006 for: (a) the Control simulation, (b) the NoTopo simulation, (c) the Kaimai simulation, and (d) the observed precipitation for the corresponding period.

the greatest detail of the precipitation field structure. The middle domain has a smaller RMS error when compared to observations due to the smoothing of the field at lower resolutions.

During the frontal period there is significant orographic enhancement along the Kaimai Range to the northeast of the Waikato River catchment in the Control simulation. When the Kaimai Range was removed this enhancement moved to the next ridge of hilltops in the centre of the North Island (this range is much lower). The biggest difference in the Kaimai simulation was the unrealistic extreme enhancement at the bottom of the parallelogram where there was a sharp change in surface altitude in the model. In the NoTopo simulation, precipitation was evenly distributed throughout the 

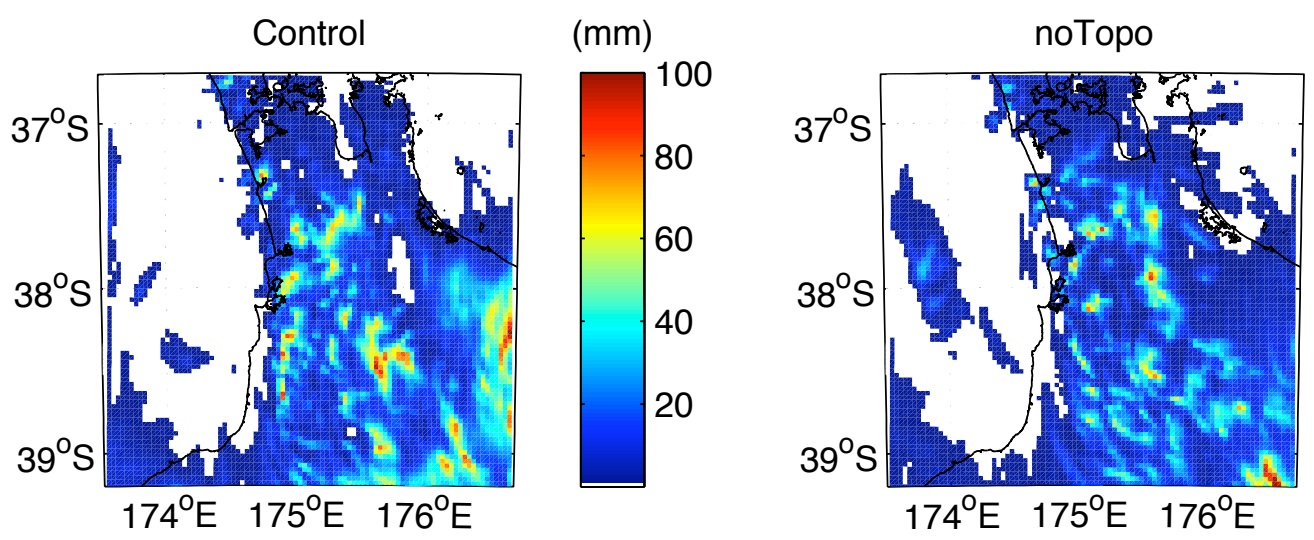

$(\mathrm{mm})$
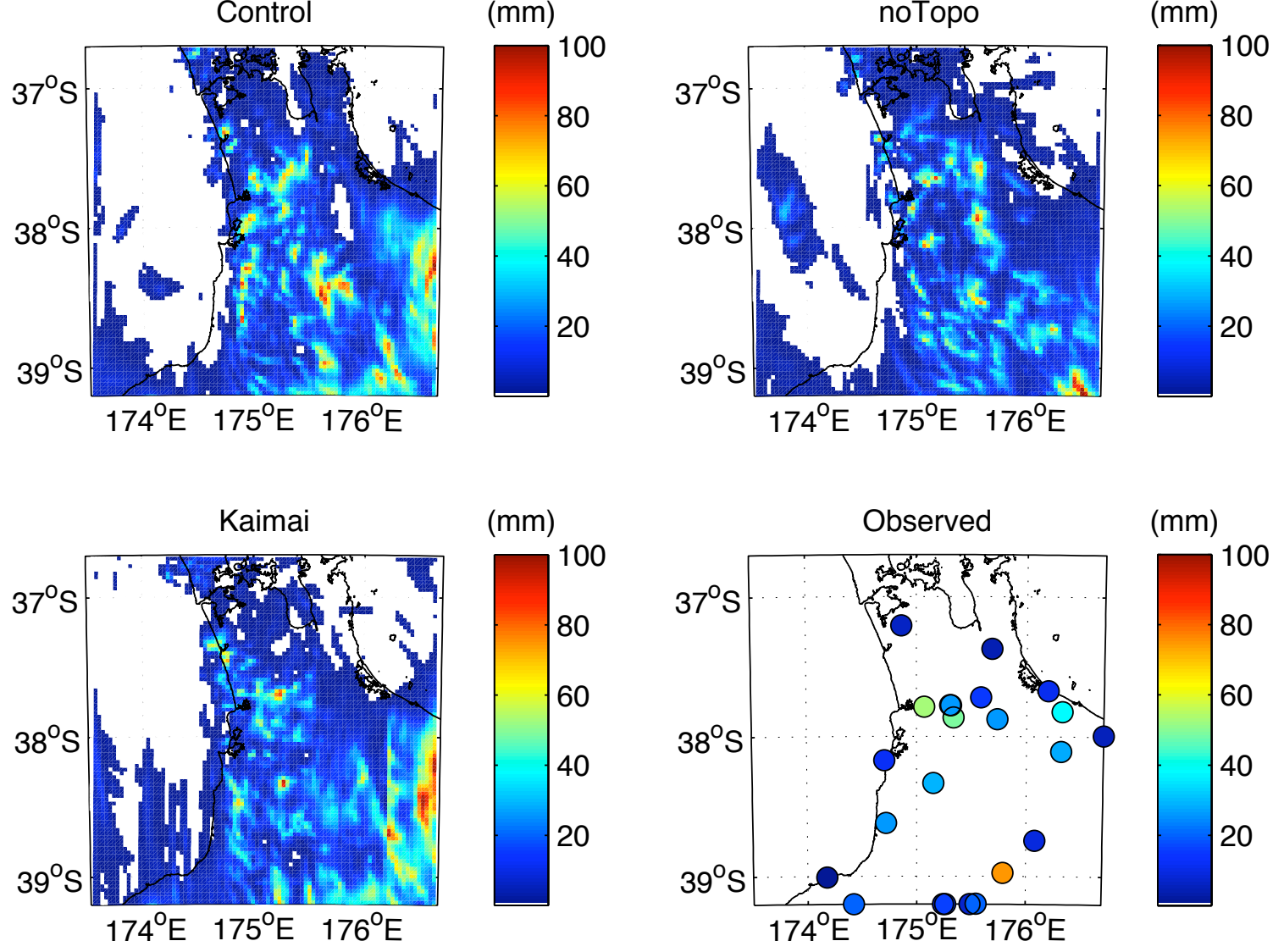

Figure 6.33 Twenty-four-hour precipitation accumulation during the convective period for the topographical simulations. This shows the total precipitation generated in the 24 hours prior to 1200 UTC 25 January 2006 for: (a) the Control simulation, (b) the NoTopo simulation, (c) the Kaimai simulation, and (d) the observed precipitation for the corresponding period.

whole domain area, whereas in the Control simulation there are precipitation peaks associated with topographical peaks throughout the North Island and sheltering in the Taumaranui area. Topography had less influence during the convective period. The artefacts of the edges of the parallelogram used in the Kaimai simulation are again clearly seen by the sudden increase in precipitation along the boundary.

Removing the Kaimai Range is clearly unrealistic but it showed that this range does shelter the central North Island from precipitation, even in the Taupo area and along the majority of the Waikato River when precipitation is occurring from the northeast. In terms of the river catchment this then means that if this range is not properly represented, then the sheltering effect within the catchment will not be correctly simulated 
and precipitation could be over-predicted.

Orographic enhancement and sheltering processes occur throughout the lower atmosphere. Therefore vertical transects of the model fields across the region at two specified times were created to see how the precipitation rates varied. There are two transects; a profile that crosses the lower Waikato and bisects the coastal hills in the west and the Kaimai Range in the east, and a perpendicular profile that runs from the west coast near Auckland to the Central Volcanic Plateau (Figure 6.34). Figure 6.34

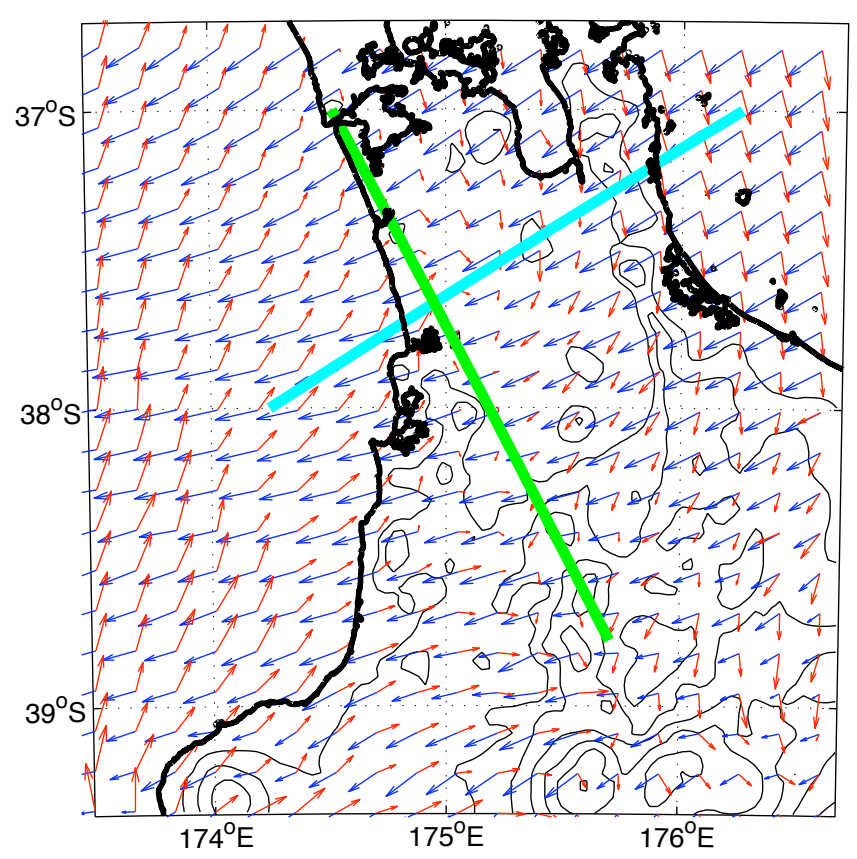

Figure 6.34 Settings for the vertical precipitation profiles. The relative surface winds (blue arrows in the direction that wind is travelling to) at 0300 UTC 24 January 2006 during the frontal period and location of the vertical profile (cyan) that bisects the coastal hills in the west and the Kaimai and Coromandel Range in the east in Figure 6.35; and the relative surface winds (red arrows) at 0600 UTC 25 January 2006 during the convective period of the vertical profile (green) that runs from the west coast near Auckland to the Central Volcanic Plateau in Figure 6.36.

also shows the surface wind fields at the time of the profiles; 0300 UTC 24 January as the frontal band was passing (Figure 6.35) and at 0600 UTC 25 January during the convective period (Figure 6.36).

So far, precipitation has been discussed in terms of the resolved and convective precipitation that is modelled to penetrate the bottom layer of the model. However, at other higher altitude model levels precipitation is defined by the instantaneous rate of falling water molecules, and is split into states of matter, water (q_rain is the rate of descending liquid water molecules), ice (q_ice), snow (q_snow) and graupel (q_graupel, heavily rimed snow particles, that can resemble tiny hail stones $\left.{ }^{\ddagger}\right)$. As ice or snow

\footnotetext{
${ }^{\ddagger}$ Glossary of Meteorology, http://amsglossary.allenpress.com/glossary/
} 


\section{Jan 20060300}

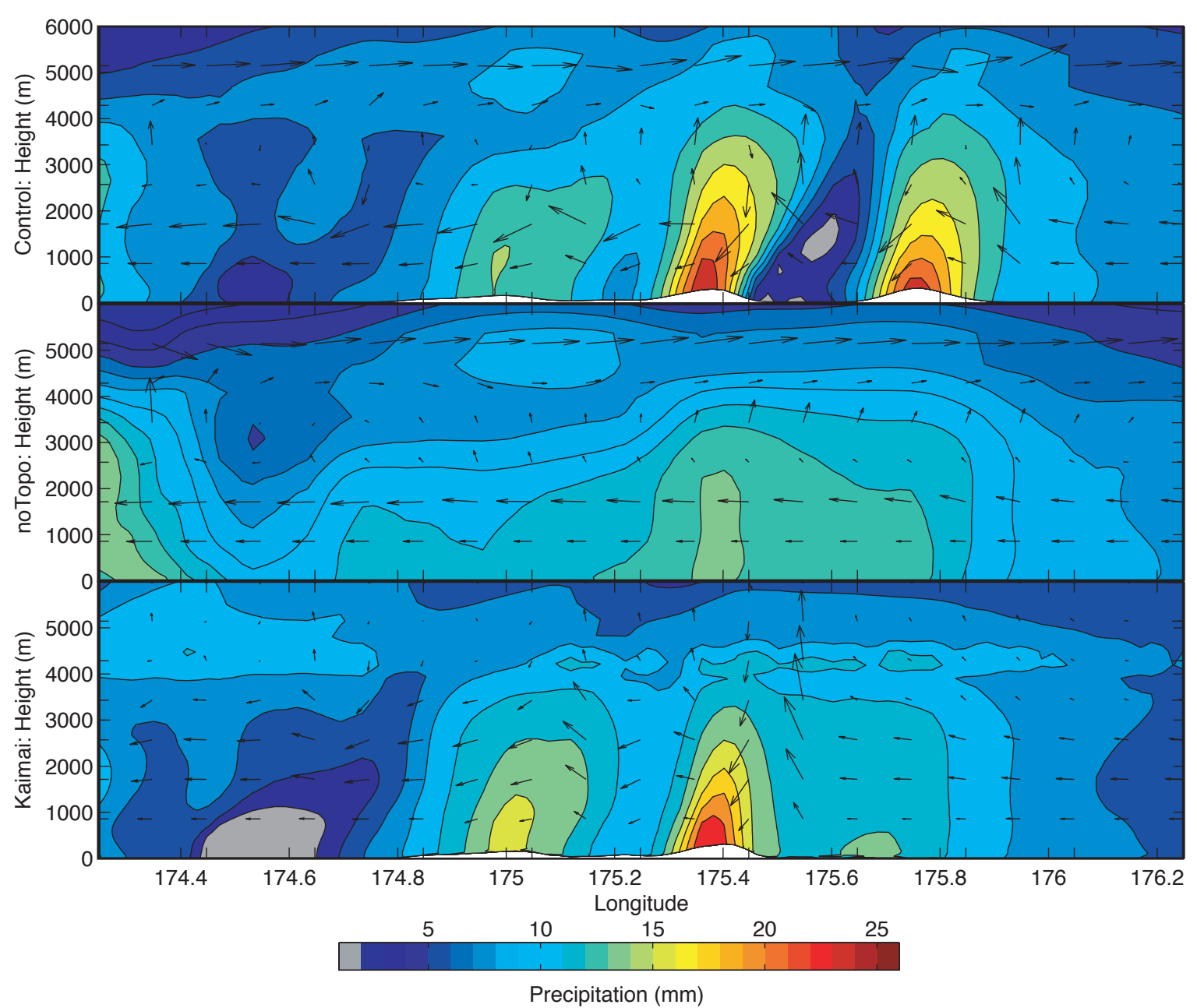

Figure 6.35 Vertical cross-section (cyan in Figure 6.34) of q_rate and relative wind speeds at 0300 UTC 24 January 2006.

molecules pass through the freezing level (the $0^{\circ} \mathrm{C}$ isotherm, which is approximately $3,000-5,000 \mathrm{~m}$ above the surface but is dependent on the season and air-mass) it melts to become liquid water. Therefore to show the downward movement of water molecules the q_rates are combined in Figures 6.35 and 6.36 and in the discussion below. These instantaneous precipitating rates are presented as $\mathrm{mm} /$ hour.

Directly above topographical peaks across the first profile line (Figure 6.35) heavier precipitation was falling in a 4,000 m column. There were strong northwesterly surface winds and strong vertical winds in the upper levels. Between the two dominant peaks and along the west coast there were dry areas. Without the topography the vertical 


\section{Jan 20060600}

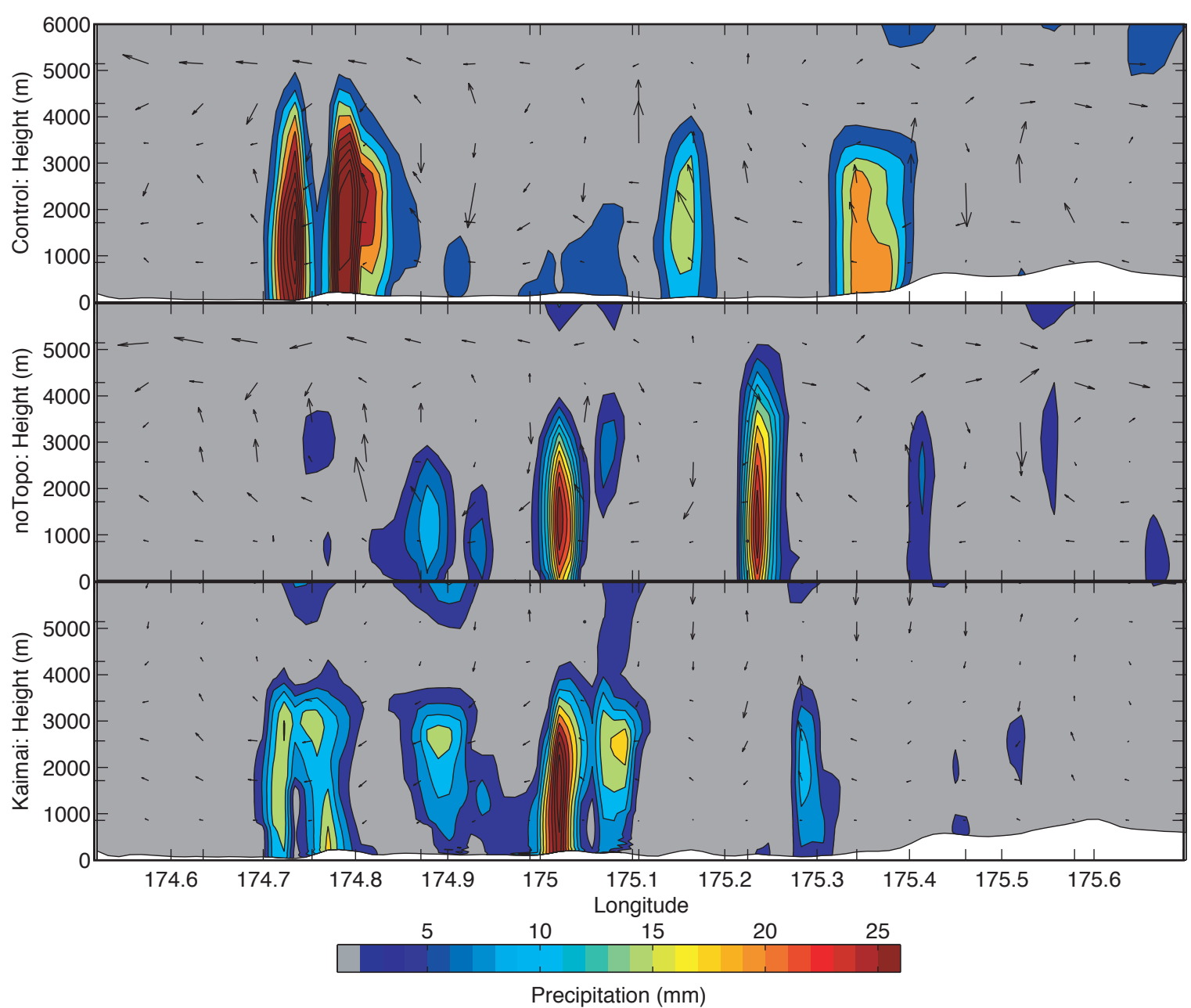

Figure 6.36 Vertical cross-section (green in Figure 6.34) of q_rate and relative wind speeds at 0600 UTC 25 January 2006.

components of the winds were significantly reduced. There was also a much wider, less intense precipitation peak over the entire land mass. In the Kaimai simulation the vertical component of the winds was reduced over the whole profile, and higher q_rates were observed above the remaining topographical features. This shows that the Kaimai Range sheltered the central North Island from the front and that it also enhanced vertical air movement over the whole area.

During the convective phase the winds in Figure 6.34 shows that there is a convergence line running from the northwest to the southeast which would have contributed to the development of convection during this period. The profile in Figure 6.36 shows 
numerous convective precipitation cells with the most intense cells (extending to 5,000 $\mathrm{m}$ above sea level) occurring on the northwest end of the profile, and another cell near the flank of Mt Ruapehu. There were fewer cells along the profile line in the NoTopo simulation and less intense cells in the Kaimai simulation, however Figure 6.33 shows that this is the result of where the transect was located. During this period of the simulations there was much less wind than during the frontal period.

Colle (2004) showed that the impact of a mountain barrier is related to the speed of the winds associated with a system. For two mountain barriers with the same slope (i.e. a low narrow barrier and a high wider barrier) the lower barrier will have more surface precipitation on the windward side than the high barrier in lighter winds. This is because the lower barrier will have more efficient warm rain processes occurring along the windward slope. If the winds are $>20 \mathrm{~ms}^{-1}$ the high barrier will receive more precipitation since it will have more extensive orographic cloud and the smaller barrier will advect more precipitation across the barrier. In the January 2006 event the winds were insufficient to carry the precipitation over the topographic barriers; however, they strongly influenced the distribution of precipitation throughout the domain area. In a cyclone with stronger winds, a greater spill-over effect could be observed.

These three simulations comparing the topographical representation in the domain area showed the effect topography has on the distribution of precipitation throughout the Waikato River catchment during a heavy precipitation event. Not surprisingly, the Control simulation generated the most realistic precipitation field during this event. However, the Kaimai simulation showed that the Kaimai Range influences precipitation generation throughout the model domain, especially the vertical winds during the frontal period. This section has shown that the topography of the catchment and surrounding areas needs to be accurately represented in mesoscale models in order to simulate realistic precipitation distributions. 


\subsubsection{Microphysics}

Resolved precipitation is generated in the WRF model by the microphysics scheme. Precipitation droplets form on a micro-scale so these sub-scale processes are generalised and parameterised for resolvable mesoscale features in the model. For example, Kessler (1969) assumed that all precipitation droplets are spherical. Since 1969 more complicated/sophisticated microphysics schemes have been developed and implemented, often optimising the scheme for a particular weather pattern (e.g. tropical convection) or a particular region (e.g. the United States of America).

There are nine microphysics schemes that are available in the WRF model. An abbreviated summary of the differences is available in Skamarock et al. (2007, page 85). Three of these schemes are frequently applied in the mid-latitudes and have been examined in this study; these are quoted as:

- Kess - Kessler scheme (Kessler, 1969): A warm rain (i.e. no ice) scheme used commonly in idealised cloud modelling studies.

- Lin - Lin et al. scheme (Lin et al., 1983): A sophisticated scheme that has ice, snow and graupel processes, suitable for real-data high-resolution simulations.

- Control - Thompson et al. scheme (Thompson et al., 2004): A new scheme with ice, snow and graupel processes suitable for high-resolutions.

The other schemes are simplified to allow for quick processing or are suitable for lower resolution simulations. However, Colle and Mass (2000) warned that using the most sophisticated and complicated microphysics scheme does not necessarily produce the most accurate precipitation distribution, especially if there is complex terrain that is not well resolved in the model.

A fourth simulation involving no microphysics (noMP) is also compared in this section. The only precipitation in this simulation is generated through the cumulus 
parameterisation. However, as there is no cumulus parameterisation in the inner domain of these simulations, the middle domain of the noMP simulation is used in the following comparisons.

The hourly precipitation time series for each simulation (Figure 6.37) were com-

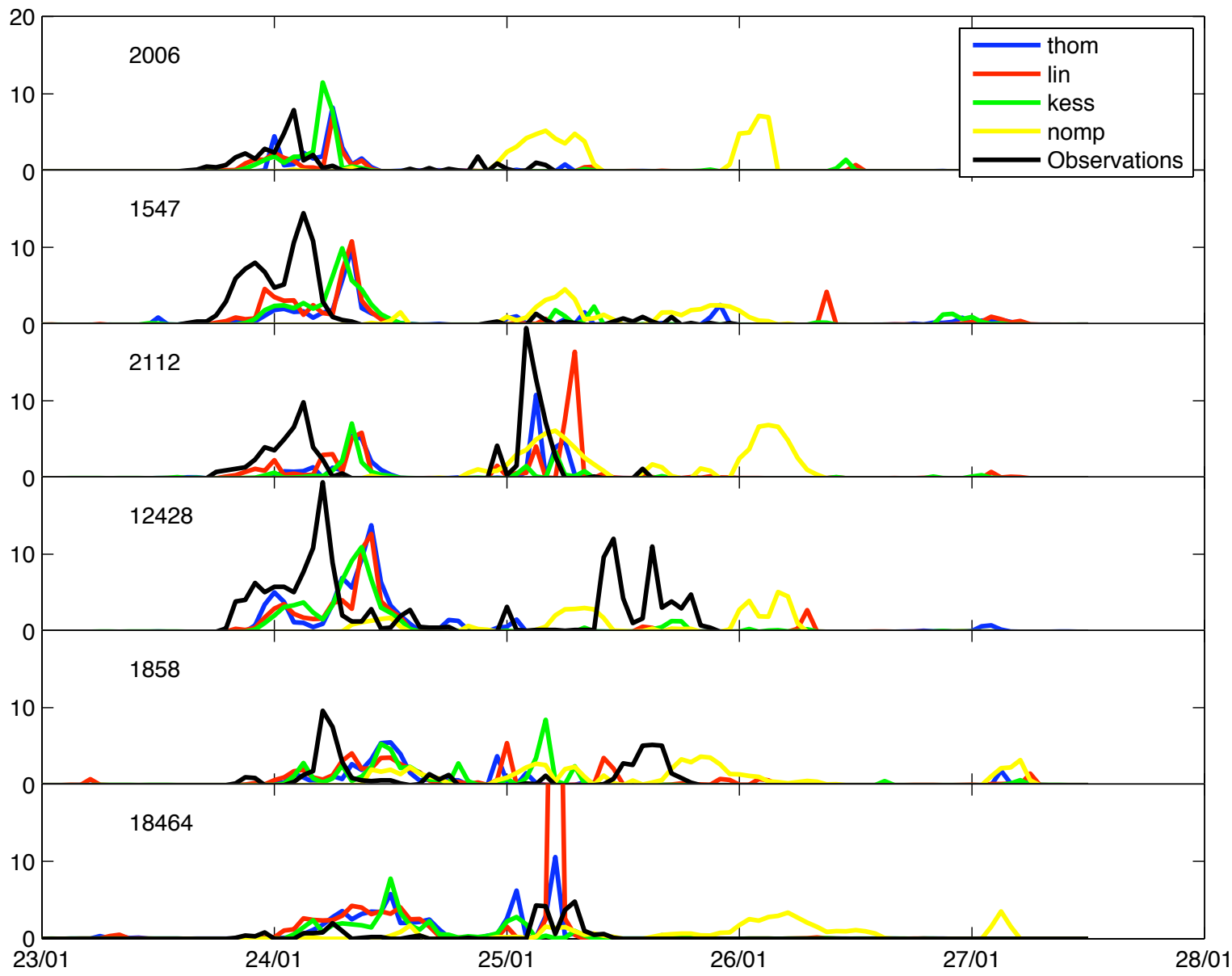

Figure 6.37 Hourly precipitation observations and simulated time series comparison from each of the microphysics simulations at selected rain gauge locations identified in Figure 6.5. Note: The Control and Observations time series are repeated from Figure 6.30 for ease of comparison.

pared, and obviously, the noMP simulation was the worst simulation. The noMP simulation contained almost no precipitation during the frontal period of the event, but produced excessive precipitation during the convective period and after the event had finished. This shows that without the microphysics more water vapour is left in the atmosphere for additional convective precipitation following the event. 
During the frontal period of the event the Control (Thompson) and Lin simulations produced very similar precipitation time series at all of the stations. However, during the convective period the Lin simulation produced more precipitation in the inland stations, and was closest to generating the peak precipitation volume at station 2112 in the lower Waikato catchment. However, at the Mt Ruapehu station the Lin simulation generated an extreme precipitation peak $(76 \mathrm{~mm})$ far in excess of the maximum measured value of $5 \mathrm{~mm}$. The Kessler microphysics produced more precipitation during the frontal period and smoother light precipitation during the convective period.

The rmse (and bootstrap confidence interval) was again calculated at each station (Figure 6.38). The most obvious feature of this figure is the extremely large confidence intervals required for two stations during the Lin simulation. The largest error is for the Chateau in the Lin simulation, which as we saw in Figure 6.37 was the result of a single hourly prediction during the convective period of the event. Apart from the few stations with especially large errors the Lin microphysics had smaller errors than the Control simulation. The Kessler simulation had slightly larger rms errors and the noMP simulation was particularly poor in the Bay of Plenty where there was heavy precipitation during the frontal period that contained no precipitation in this simulation (see Figure 6.37).

During the frontal period (Figure 6.39) and the 24-hour period prior to 1200 UTC 25 January in Figure 6.40. the Lin simulation produced more evenly distributed lighter precipitation, with almost no precipitation over Mt Egmont/Taranaki, and lighter precipitation on each of the two ridges near the northern side of the Waikato River catchment compared to the Control run. The Kessler simulation generated a similar precipitation distribution to the Control but with heavier precipitation along the ridges.

In the convective period (Figure 6.40) the noMP simulation produced widespread moderate precipitation over the entire North Island and to the northwest. When microphysics was included there was widespread light precipitation over the North Island 

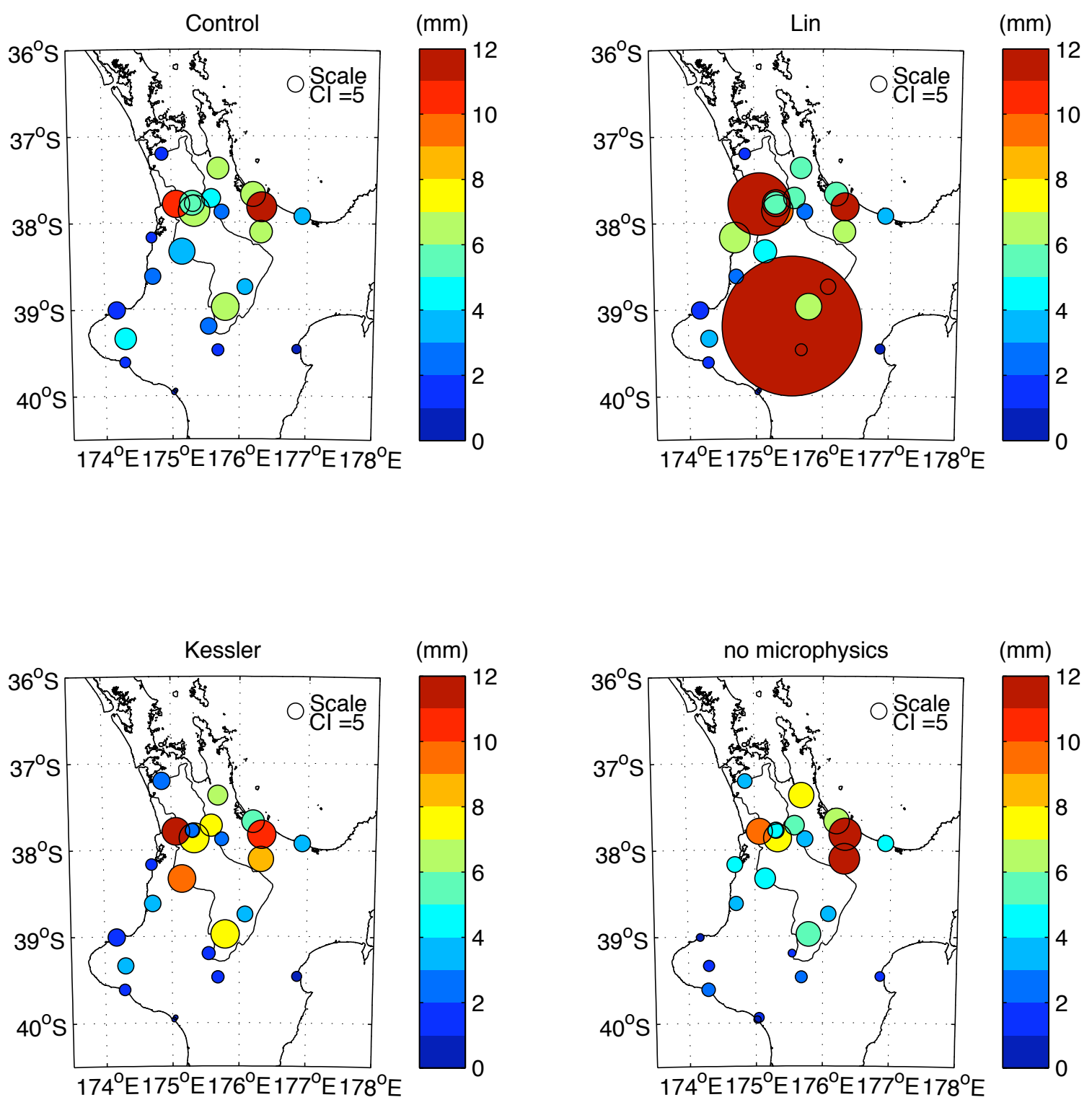

Figure 6.38 The rmse for individual hourly precipitation over the event for each of the four microphysics schemes. Note: the Control rmse is the same as Figure 6.31 but has been included for ease of comparison.

with small convective cells of heavy precipitation. In this period the Lin simulation produced the heaviest precipitation and the Kessler produced the smoothest distribution. The Lin simulation was the only simulation to simulate heavy precipitation southwest of the catchment area.

As expected overall, the noMP simulation performed the worst, completely missing the frontal periods and predicting evenly distributed widespread moderate precipita- 

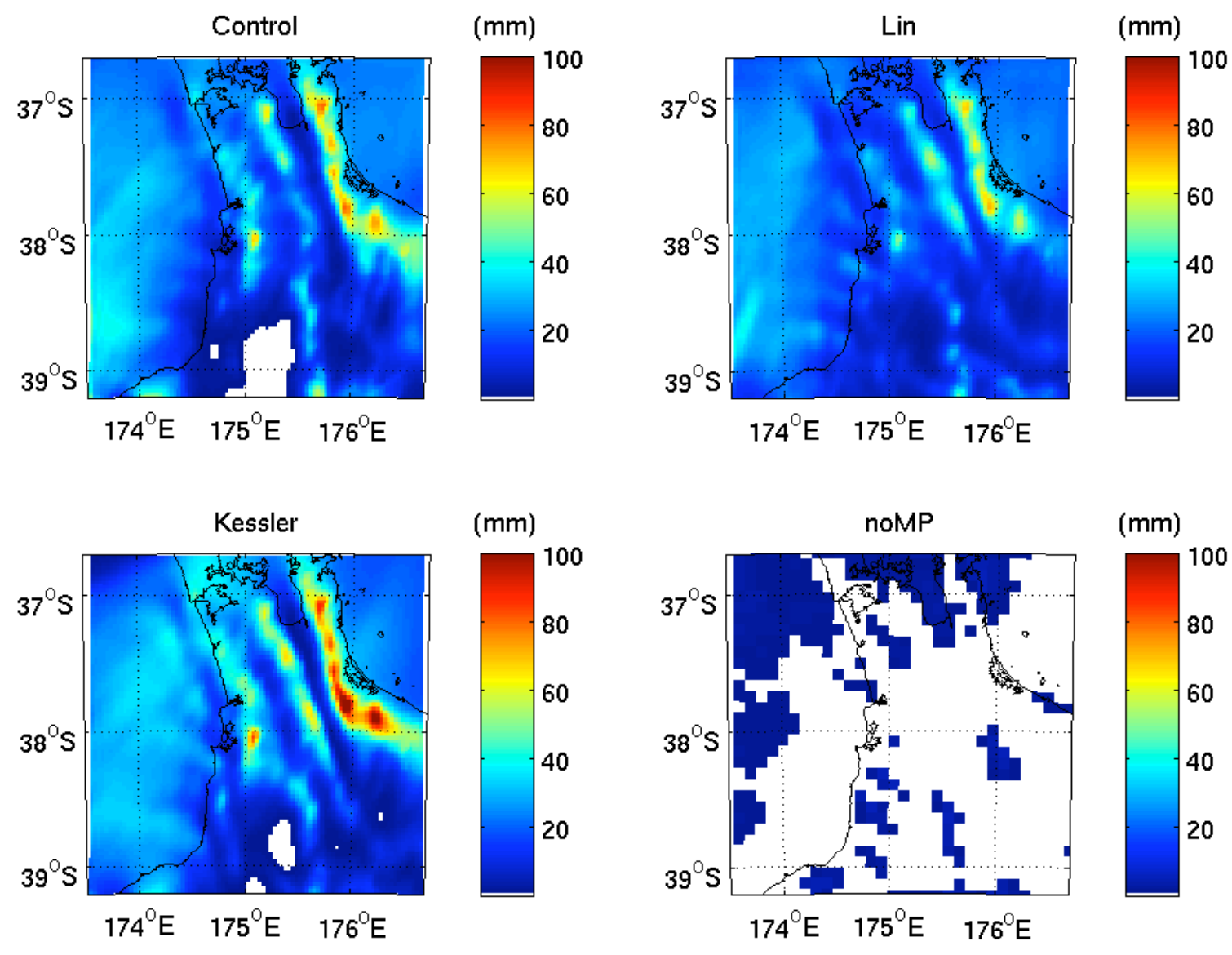

Figure 6.39 Six-hour precipitation accumulation during the frontal period for the microphysics simulations. This shows the total precipitation generated in the six hours prior to 0900 UTC 24 January 2006 for: (a) the Control simulation, (b) the Lin simulation, (c) the Kessler simulation, and (d) the noMP simulation. Note: The Control simulation is identical to Figure 6.32 but has been included here for ease of comparison.

tion throughout the convective period. This setting is not recommended for weather modelling but does illustrate how much of the precipitation is generated through the cumulus parameterisation.

The Thompson microphysics produced a precipitation field that appears to be a compromise between the Lin and the Kessler microphysics schemes. The Kessler simulation produced the heaviest precipitation over topographical features during the frontal period but also produced the smoothest distribution during the convective period. Meanwhile the Lin microphysics smoothed the frontal period (Colle et al., 2005, Lin microphysics apparently depletes cloud water over topographic crests by over- 

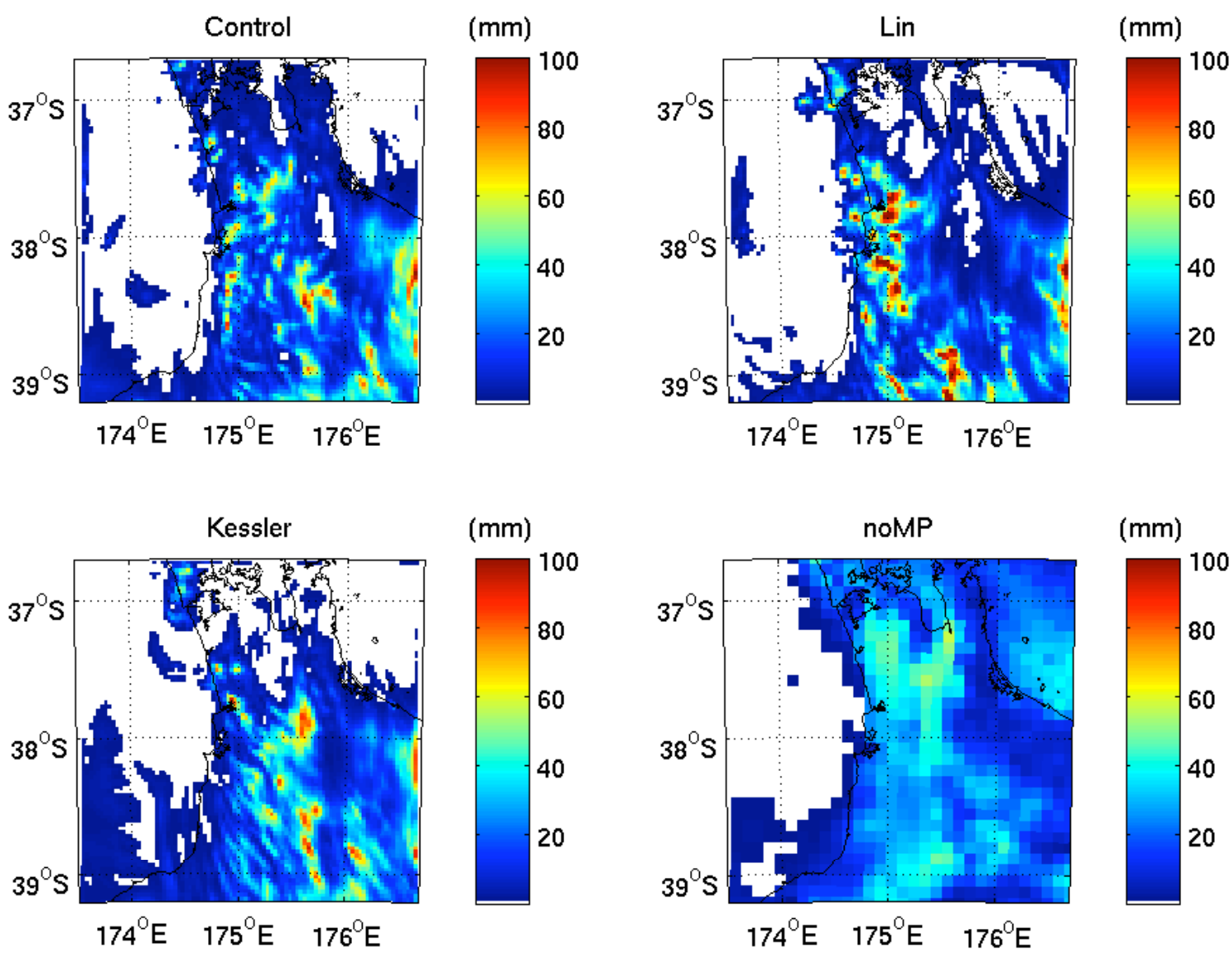

Figure 6.40 Twenty-four-hour precipitation accumulation during the convective period for the microphysics simulations. This shows the total precipitation generated in the 24 hours prior to 1200 UTC 25 January 2006 for: (a) the Control simulation, (b) the Lin simulation, (c) the Kessler simulation, and (d) the noMP simulation. Note: The Control simulation is identical to Figure 6.33 but has been included here for ease of comparison.

generation of snow) and produced the most cellular convection during the convective period. The Lin microphysics scheme also produced extremely large spot differences that were unrealistic.

In both periods, the Thompson microphysics in the Control simulation produced the intermediate amount of precipitation and has the most balanced scheme for generating precipitation during events. All schemes generated insufficient lagged precipitation in the frontal band and over-estimated precipitation during the convective period the following day. The difference between any two of these model runs is less than the difference between any of them and the observations. However, the warm rain Thompson 
scheme is the most appropriate microphysics scheme to use within this catchment.

\subsubsection{Cumulus parameterisation}

Cumulus convection also occurs at sub-grid scales and is normally parameterised in models. Unlike the precipitation described by the microphysics, this convective precipitation occurs in atmospheric structures that are unresolvable in the grid. In highresolution simulations $(<10 \mathrm{~km})$ it is expected that the convective eddy can be resolved explicitly and therefore cumulus parameters are not regularly applied (e.g. Colle and Mass (2000) did not use cumulus parameterisation in their 4- and 1.4-km nests). Cumulus parameters are not theoretically valid below 5-km resolution (Skamarock et al., 2007), but Deng and Stauffer (2006) noted that excluding the convective parameterisation meant that response to convection on grids of $4-\mathrm{km}$ as the forced updrafts are unrealistically strong.

To examine the effect of the cumulus parameterisation in the inner domain a ' $\mathrm{Cu}$ mulus' simulation was conducted. This only differs from the Control simulation in that there is cumulus parameterisation in the $3-\mathrm{km}$ inner domain. This means that the outputs in the 27- and 9-km domains are identical. All cumulus parameterisation in this study is the modified Kain-Fritsch parameterisation. There are numerous other cumulus parameterisations available; however, this is the most popular for mid-latitude locations where convection is less vigorous.

In complex terrain, it was suggested by Colle et al. (2003) that applying cumulus parameters in high-resolution domains $(3-\mathrm{km})$ could improve precipitation simulation better than increasing the resolution over a limited area. It was also noted that if convective processes are triggered too often within the outer domains, the troposphere stabilises and prevents resolved convection from developing within the inner nests (Colle et al., 2003; Deng and Stauffer, 2006). The convective schemes have since been updated to remove this problem (Anderson et al., 2006).

The inclusion of the cumulus parameterisation in the inner domain had almost 
no effect on the hourly time series of the non-convective frontal period (Figure 6.41). However, more precipitation was generated with the cumulus parameter during the

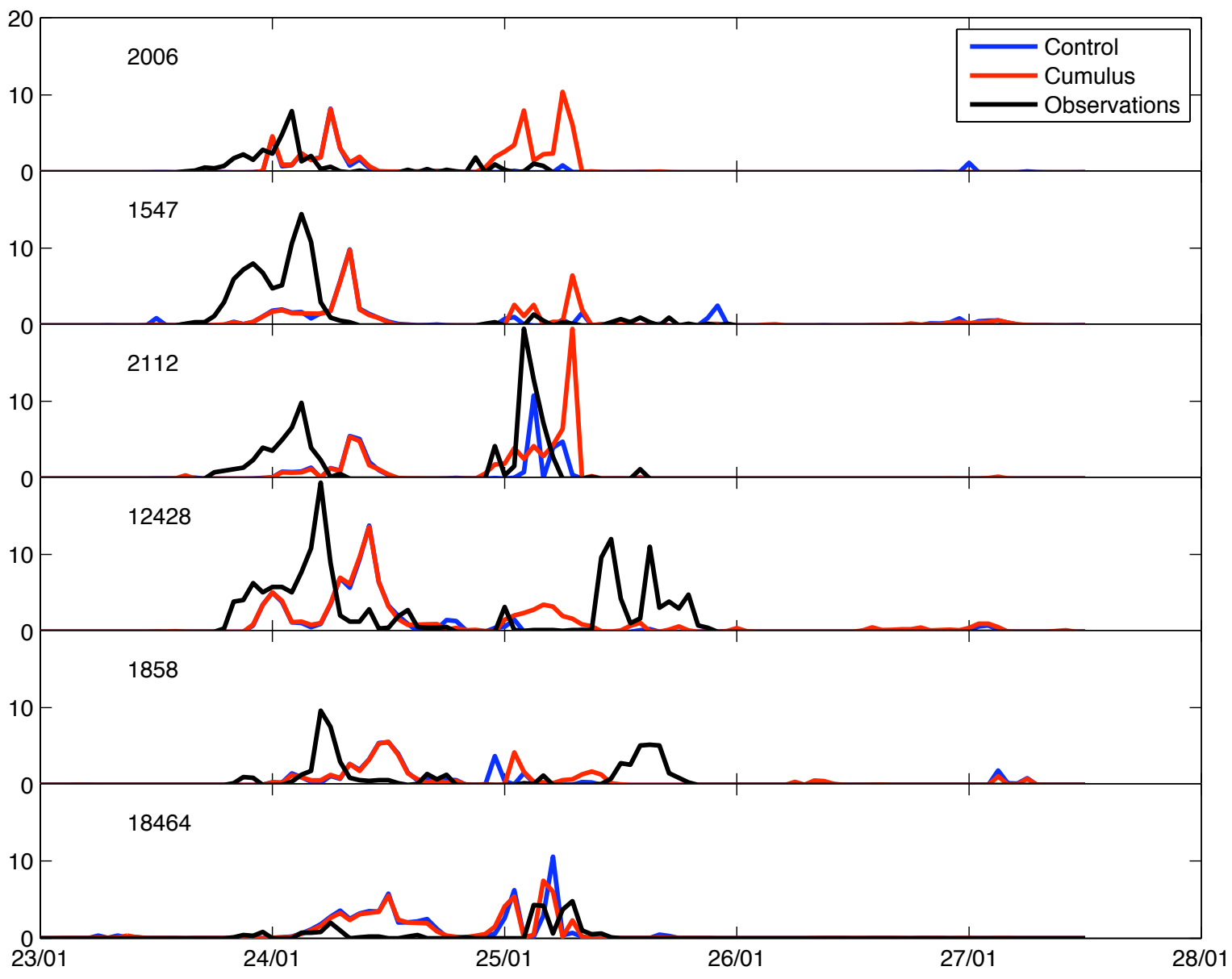

Figure 6.41 Hourly precipitation observations and simulated time series comparison from each of the cumulus simulation at selected rain gauge locations identified in Figure 6.5. Note: The Control and Observation time series are repeated from Figure 6.30 for ease of comparison.

convective period, with excessive precipitation generated in the north, and neither simulation generated the precipitation observed on Mt Ruapehu.

The rmse between the simulated hourly time series and observations is shown in Figure 6.42. This shows that the confidences of the rms errors for each station remain constant but at three stations the magnitude of the error is increased in the Cumulus simulation. At the remaining stations the rmse is the same from both simulations.

Statistically there is little difference between the precipitation generated in the 

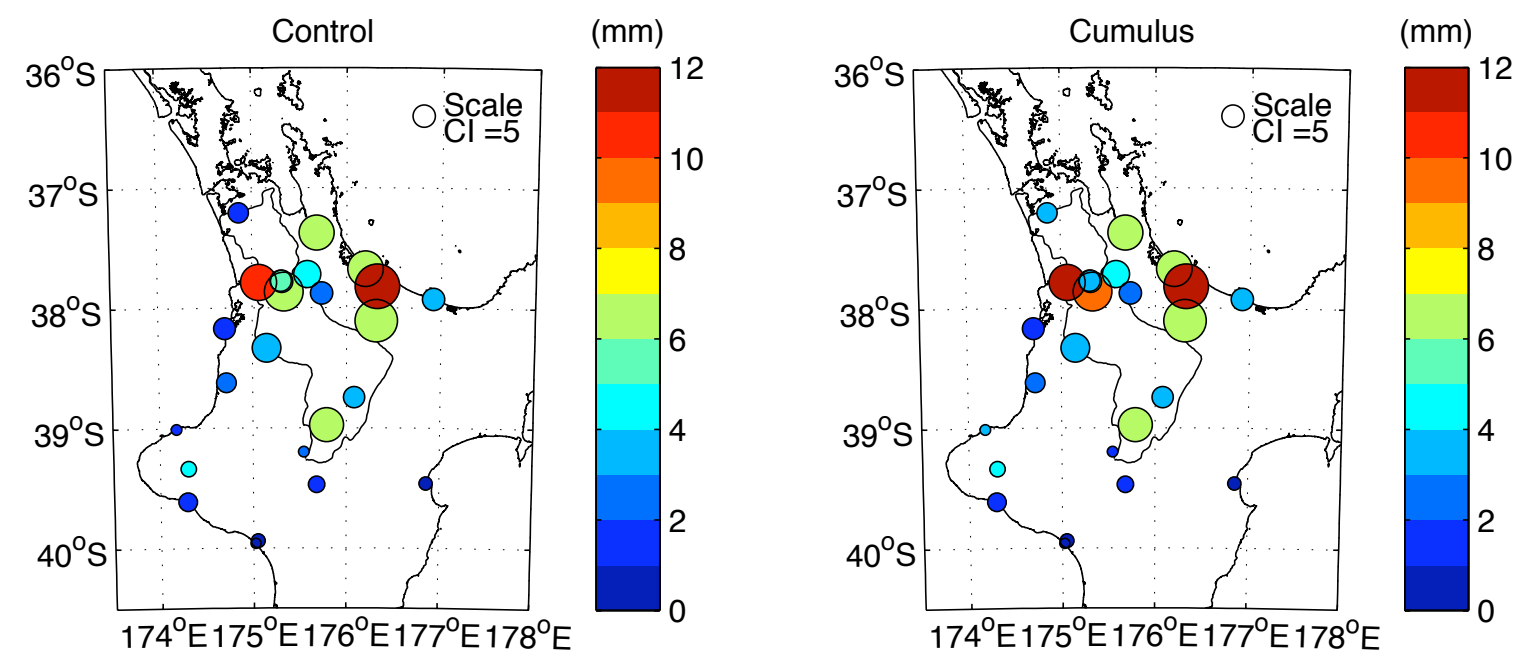

Figure 6.42 The rmse for individual hourly precipitation over the event for the Cumulus simulation. Note: the Control rmse is the same as Figure 6.31 but has been included for ease of comparision.

Control and Cumulus simulations. However, the precipitation accumulation has been plotted for the convective period in Figure 6.43 (the frontal period is not presented here
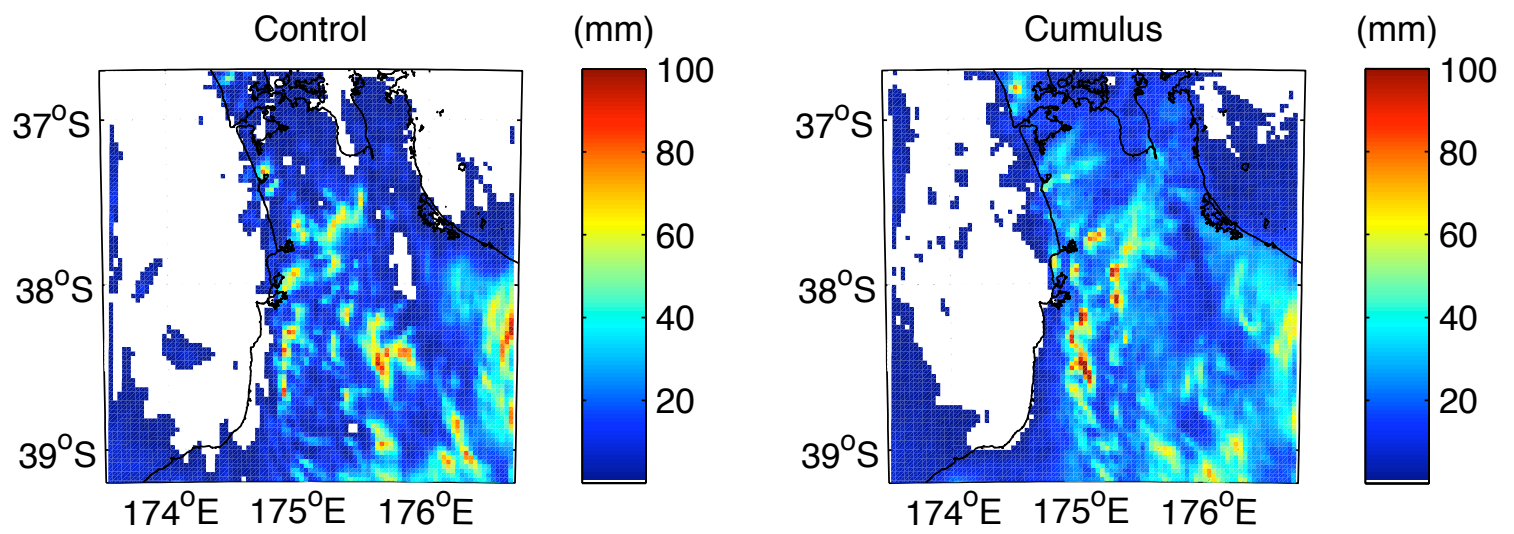

Figure 6.43 Twenty-four-hour precipitation accumulation during the convective period for the cumulus simulations. This shows the total precipitation generated in the 24 hours prior to 1200 UTC 25 January 2006 for (a) the Control simulation, (b) the Cumulus simulation. Note: The Control simulation is identical to Figure 6.33 but has been included here for ease of comparison.

as there is no visual difference). During the convective period the cumulus simulation places the larger volume precipitation cells closer to the west coast, and then generated lighter, more evenly distributed precipitation in the southeast of the domain area. The Control model has more high volume precipitation cells in the centre of the Waikato 
River catchment.

Including the cumulus parameterisation in a high-resolution domain does not alter the amount of precipitation generated during the frontal period of the event. It does, however, create narrower bands of heavy precipitation and more widespread light precipitation during convective conditions. In general little value is added to the simulation by including the extra cumulus parameterisation at high resolution, but it could be used to increase the precipitation totals if it is important to not under-predict the quantity of precipitation over an area, although the timing and location would remain physically unconstrained.

\subsubsection{Model resolution}

The first consideration when planning a weather simulation or forecast is the set-up of the model domain. This led to a small investigation of the differences in precipitation generated on slightly different domains. Over the period of this study there were four domains defined as in Table 6.4, and this section is a comparison of the precipitation generated in each of these for the January 2006 event.

Table 6.4

Domain details for model resolution simulations. This gives details of the number of vertical levels, the grid spacing in each of the three domains and the number of grid points in the inner domain for each of the four simulations in this subsection.

\begin{tabular}{|c|c|c|c|}
\hline Model Run & Vertical levels & Grid spacing & Grid points \\
\hline \hline Control & 27 & $21,9,3-\mathrm{km}$ & $99 \times 93$ \\
\hline Vertical & 54 & $21,9,3-\mathrm{km}$ & $99 \times 93$ \\
\hline Resolution & 27 & $36,12,4-\mathrm{km}$ & $74 \times 70$ \\
\hline Boundary & 27 & $36,12,4-\mathrm{km}$ & $99 \times 93$ \\
\hline
\end{tabular}

To estimate the value of increasing the vertical resolution (which will significantly increase the run time, but allow for smaller vertical shifts to air masses and water molecules) a Vertical simulation was run with 54 vertical levels instead of the Control's 27 vertical levels. The other simulations in this section have domain grids at 36-, 12-, 
and $4-\mathrm{km}$ to investigate the effect of this slight change in resolution. One of these simulations contains the same number of grid points as the Control extending the boundary beyond the Control simulation boundary (Boundary), and the other reduced the number of grid points to maintain the boundary position of the Control simulation (Resolution).

The realism of model output generally increases with resolution. However, when the skill is validated using methods such as the RMSE a better skill may be achieved on a lower resolution grid, although the forecast maybe less useful. During the convective period of these simulations the middle domain had a lower average RMSE than the inner domain as it produced widespread moderate precipitation instead of the intense precipitation cells shown in the inner domain (similar effects were also noted by Colle et al. (2000)). Therefore mis-location of convective cells was not penalised as harshly; however, the higher resolution grid provided more detail as to the likely structure of precipitation, i.e. heavy scattered showers versus widespread light precipitation. Colle et al. (2003) showed that the skill of different resolutions was dependent on the level of precipitation, i.e. lower resolution has better skill for light rain, and high resolution for heavy rain.

The hourly time series comparison (Figure 6.44) shows more variation between the simulations than seen in the previous sections. Changing the vertical resolution of the simulated domains had the smallest effect in this set of simulations but like the Kaimai and Lin simulations very heavy precipitation was generated on Mt Ruapehu during the convective period. The largest difference is in the Boundary simulation that generated more prefrontal precipitation at station 1547, and heavier precipitation in the convective period for stations 2006 and 2112. The Resolution simulation generated more prefrontal precipitation at station 1547 and more intense precipitation cells during the convective period in the central North Island.

Figure 6.45 compares the rmse for each of the simulations at each station. Increasing the vertical resolution increased the rmse at stations within the Waikato River catch- 


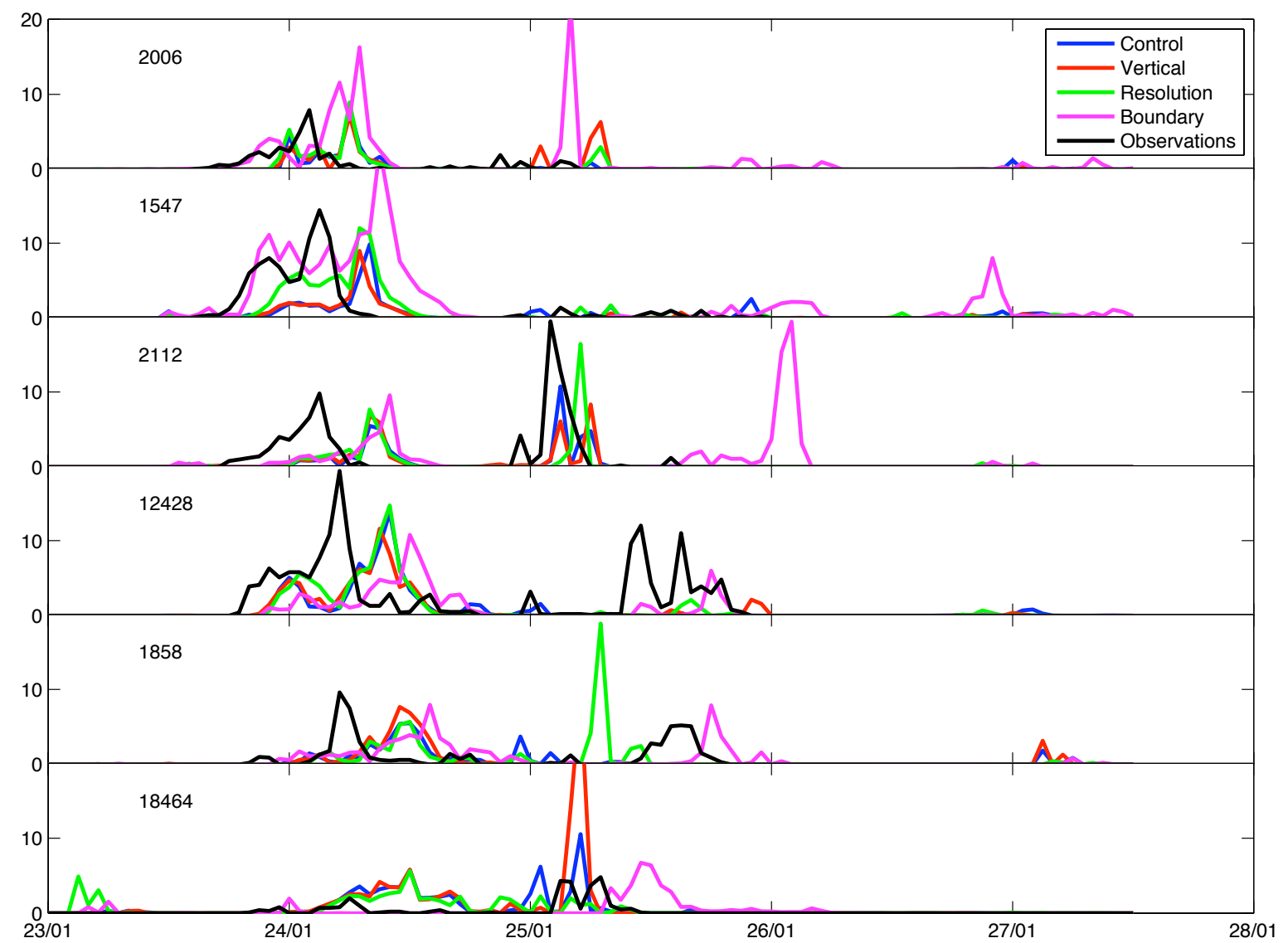

Figure 6.44 Hourly precipitation observations and simulated time series comparison from each of the domain resolution simulations at selected rain gauge locations identified in Figure 6.5. Note: The Control and Observation time series are repeated from Figure 6.30 for ease of comparison.

ment but did not affect the confidence interval. Changing the resolution of the model increased the errors at inland stations, but it lessened the errors in the Bay of Plenty. Changing the boundary position increased errors in the northern half of the domain area but still did not change the confidence interval. In general the smallest differences were in the Control simulation.

The Vertical simulation generated more precipitation over the Kaimai Ranges during the frontal period (Figure 6.46) than the Control run, and the Resolution simulation increased the spatial extent and intensity of generated precipitation during the frontal period. In the Boundary simulation there is more intense precipitation in a band across the northwest of the domain, and there is more enhancement over large topographic 

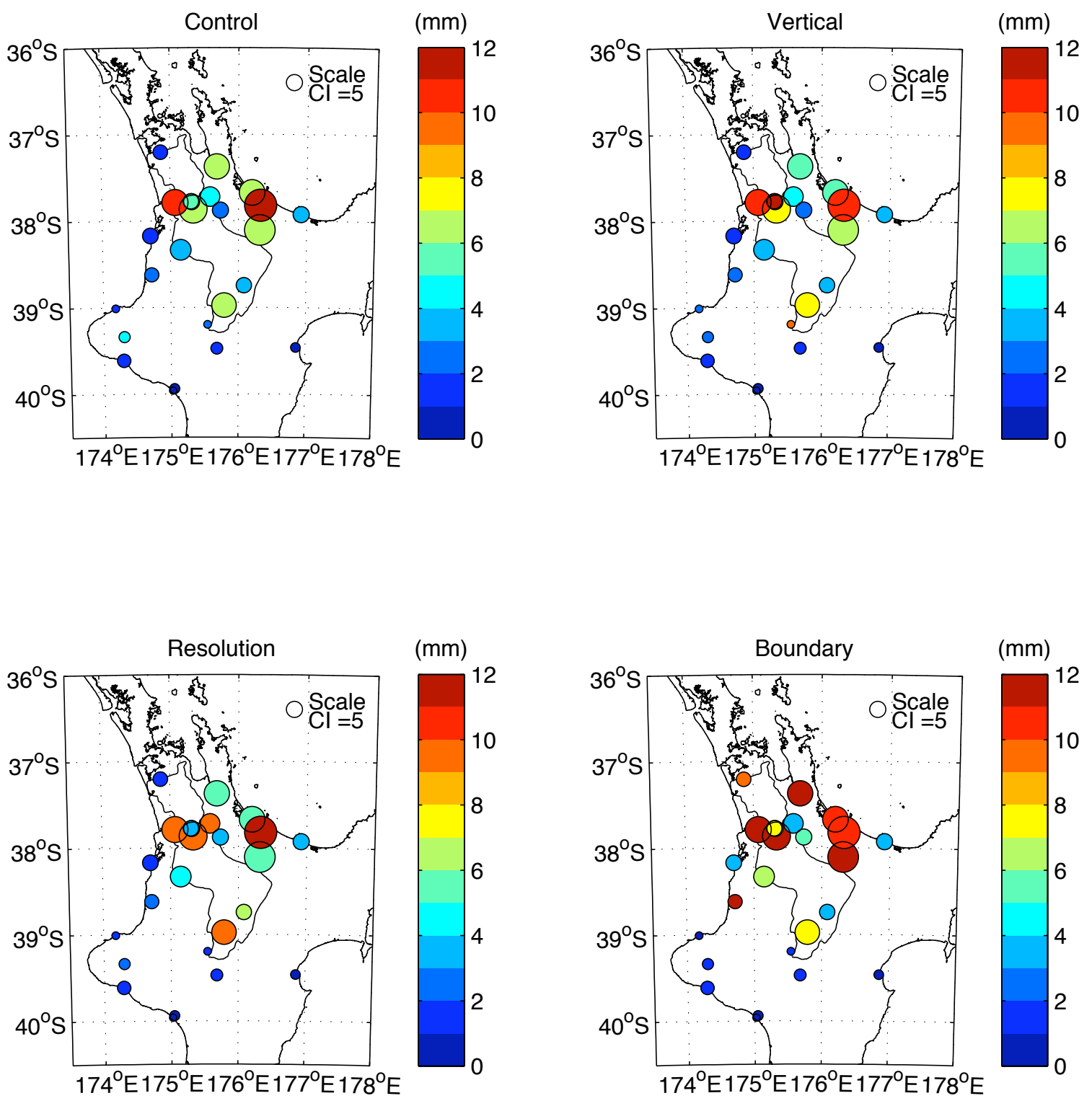

Figure 6.45 The rmse for individual hourly precipitation over the event for each of the domain resolution simulations. Note: the Control rmse is the same as Figure 6.31 but has been included for ease of comparison.

features in the south of the domain.

Again the Boundary simulation had the most significant difference during the convective period (Figure 6.47), generating multiple extensive cells of heavy precipitation in the southeast half of the domain. The Resolution simulation also generated more extensive smoother precipitation cells during this period, although the distribution is consistent with the Control simulation. Comparatively the Vertical simulation had 

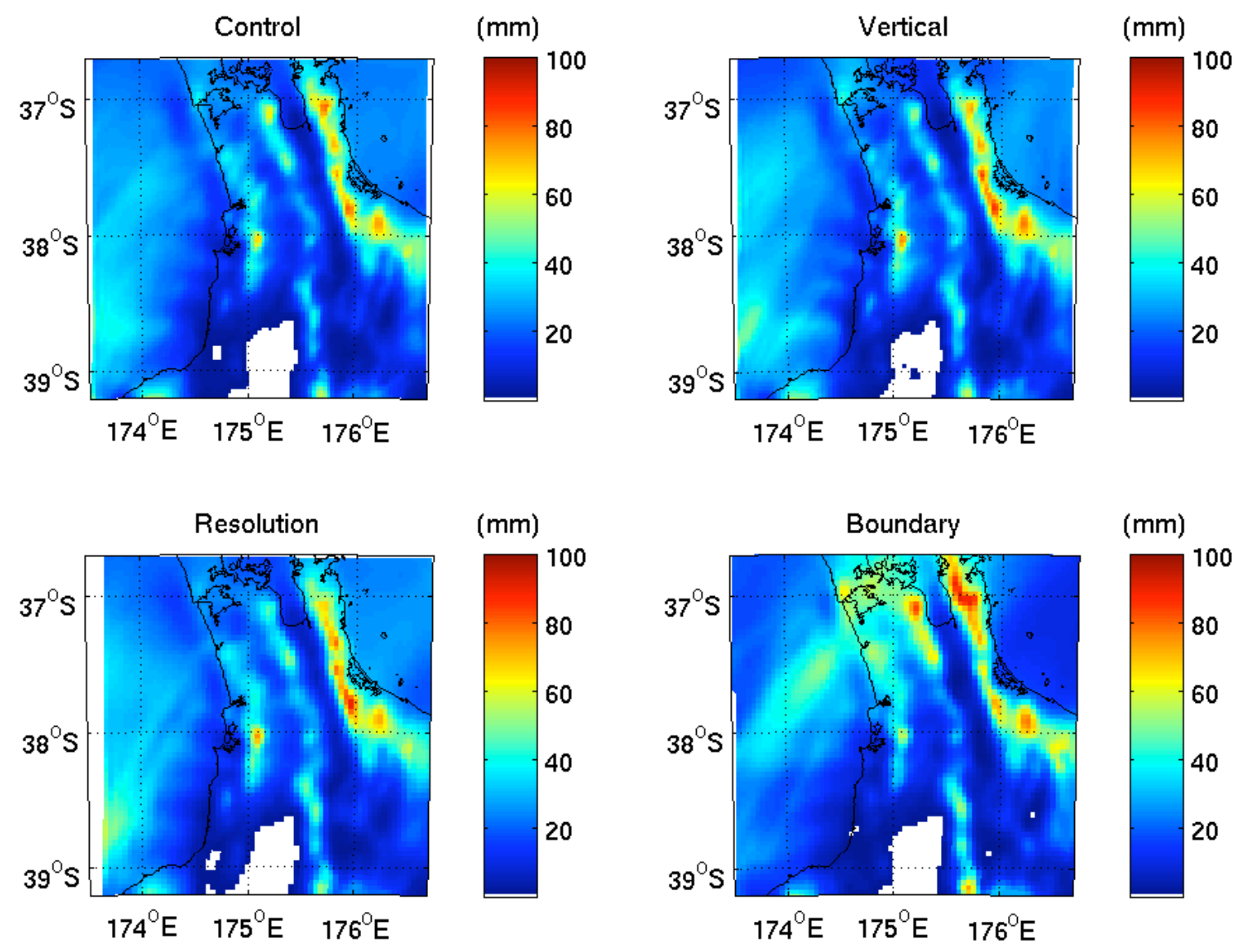

Figure 6.46 Six-hour precipitation accumulation during the frontal period for the domain resolution simulations. This shows the total precipitation generated in the six hours prior to 0900 UTC 24 January 2006 for (a) the Control simulation, (b) the Vertical simulation, (c) the Resolution simulation, and (d) the Boundary simulation. Note: The Control simulation is identical to Figure 6.32 but has been included here for ease of comparison.

smaller variations although more of the convective cells were located in the south of the domain.

The implication of this section is that the domain settings have more influence on precipitation generation than the various physics options available in the model. Increasing the vertical resolution increased the amount of orographic enhancement produced by the model. Colle and Mass (2000) identified that higher vertical resolution is useful when there is spill-over on a mountain range. In this case the small difference could mean that there was limited spill-over, or it could be part of the under-prediction error. 

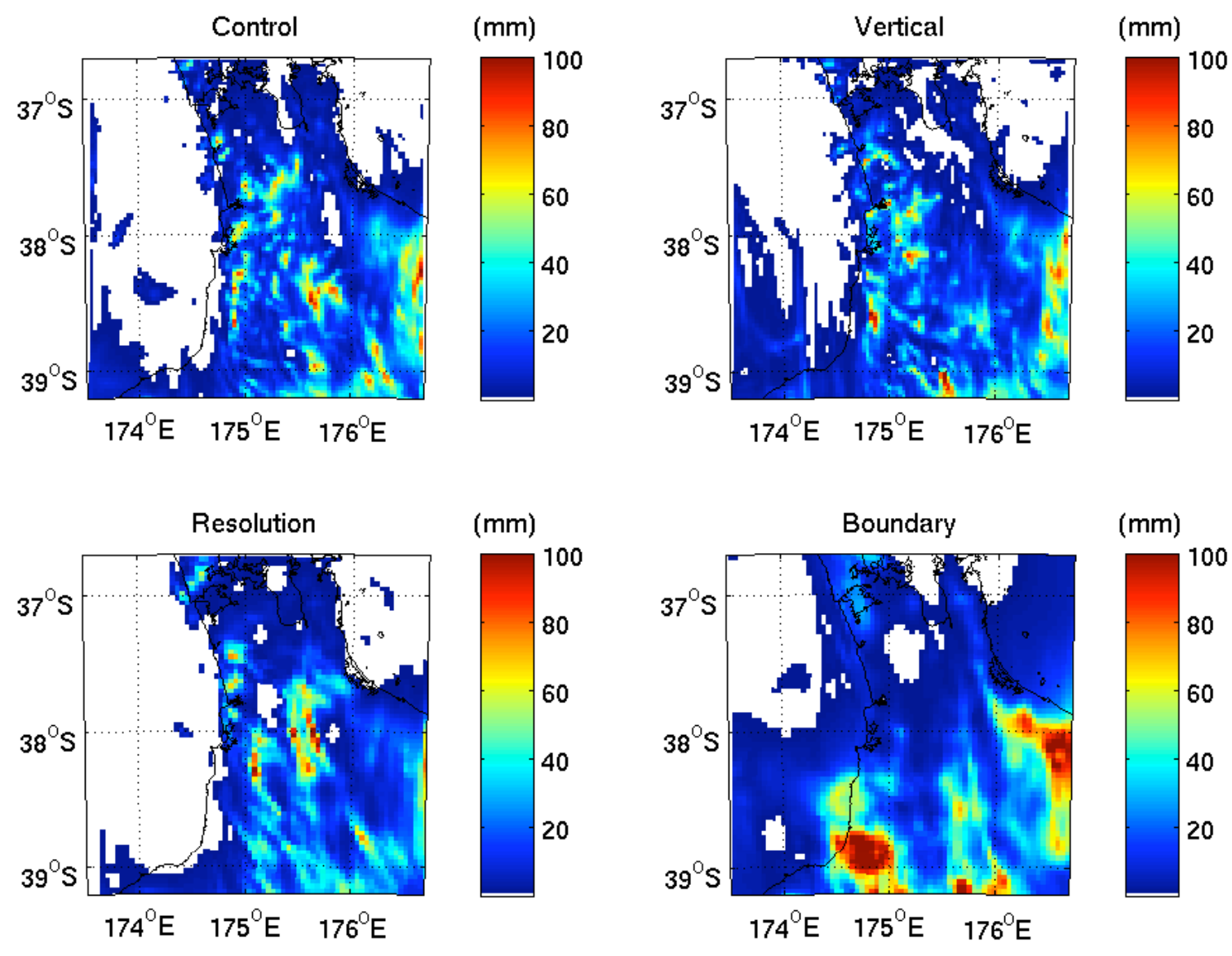

Figure 6.47 Twenty-four-hour precipitation accumulation during the convective period for the domain resolution simulations. This shows the total precipitation generated in the 24 hours prior to 1200 UTC 25 January 2006 for (a) the Control simulation, (b) the Vertical simulation, (c) the Resolution simulation, and (d) the Boundary simulation. Note: The Control simulation is identical to Figure 6.33 but has been included here for ease of comparison.

Increases to the grid size will have a smoothing effect on precipitation generation. The largest variations to the simulation were in the Boundary run. The larger domain altered the timing of the front, the pre-frontal precipitation and the structure and location of the convective cells. The only difference between this simulation and Resolution was the boundary constraints and this illustrates the importance of accurate global model input to constrain the mesoscale model, and the choice of a domain that is sufficiently large that boundary effects do not influence the development of precipitation in the inner domain. Unfortunately increasing the domain size increases the amount of data generated and the time needed to process the data. This effect could 
have been due to the nested domain decoupling from the parent domain as occurred in Drost et al. (2007). This discussion of the boundary placement during this event is continued in section 7.2.2.

\subsection{Discussion}

This chapter has shown that a mesoscale model can significantly improve the distribution and quantity of precipitation predicted during a heavy precipitation event. However, the skill at simulating the observed precipitation is dependent on the type of weather system approaching, the model options, the domain set up and the quality of the input data.

Precipitation is recognised as one of the most difficult atmospheric parameters to model. Precipitation is so difficult because of its spatial and temporal variability. This also means that a lot of research has been conducted on the best way to simulate precipitation in numerical weather models. There are many different schemes that have been developed and this research could not test every possibility. A selection of parameters were examined based on the literature and WRF user's guide (Skamarock et al., 2007) in an effort to determine the best options for future operational forecasts in the Waikato River catchment.

None of the simulations managed to generate the total amount of precipitation with the best simulation generating $92 \%$ of the observed precipitation. Zängl (2007) also noted that the WRF model generally under-predicted precipitation amounts in mountainous regions. Further examination of the January case study and altered topography files showed that when topography was scaled to sea level, the model generated widespread lighter precipitation, which is exactly what was seen in Chapter 5. The representation of the topography has more influence in the distribution of frontal precipitation than convective precipitation. However, in the events that contained a large portion (>50\%) of convective precipitation (October 2005 and October 2006) the representation of topography had a greater influence in increasing the total precipitation 
generated during the event.

Even with the 'perfect' GFS analysis files derived from data assimilation of real observations over the previous three hours, the timing of the cold front in the January 2006 event was four hours late. The mesoscale model could not compensate for this error, but the Boundary simulation showed that a slight alteration of the boundary conditions could significantly alter the development of the precipitation in this front for the inner domain. This illustrates that the selection of the model boundary can have a significant impact on the precipitation generated in the model run. Therefore, as stated in the operation manual, the boundary needs to encompass the full extent of influence of the approaching system. This timing error also indicates that the accuracy of the mesoscale model is ultimately limited by the accuracy of the global model.

The examination of microphysics and cumulus parameters showed that a small difference could be obtained by altering these options. Thompson microphysics gives the best representation of both the frontal and convective stages of the January event and therefore it could be expected to present the best average precipitation forecasts over all conditions observed in the Waikato river catchment. Gallus and Bresch (2006) identified that while the peak precipitation rates are dependent on the microphysics used, they thought that the total volume of precipitation might be more dependent on the dynamical core of the model, which was not tested in these experiments.

The mesoscale model adds significant value to quantitative precipitation forecasts during heavy precipitation events in the Waikato River catchment. However, sub-grid convection cannot be accurately positioned or timed within the domain as the model cannot physically constrain it. This accounted for up to $50 \%$ of the total precipitation in two of the case studies, yet the area averaged precipitation is still $10-20 \%$ below the observed precipitation value, similar to under-prediction values noted by Zängl (2007) and Milbrant and Yau (2001). Despite this, the mesoscale model significantly improves the quantitative precipitation forecasts provided from a global model by distinguishing between areas of the catchment that receive precipitation and taking into consideration 
the effects of topography that have been shown to significantly influence the distribution of precipitation. 
174

Dravitzki, 2009 
CHAPTER 7

\section{DYNAMIC DOWNSCALING}

The WRF mesoscale model was used to dynamically downscale a complete two-year period of weather to provide a model climatology in data-sparse subcatchments and validate the mesoscale model's ability to predict precipitation over the complete range of weather systems affecting the Waikato River catchment. Computer resources limited the simulation to a two-year period, but this should include most types of weather and also coincides with the time period of the GFS archive.

The climatology of the Waikato River catchment described in Chapter 3 is limited by the lack of observational data, particularly in high altitude and sparsely populated areas. The downscaling is used to approximate the precipitation climate in the datasparse regions, and therefore quantify the average amount of precipitation in each subcatchment. The simulation is validated against existing observational data. This technique of developing a climatology was also used in Ibbitt et al. (2001, a 29-day simulation over New Zealand's South Island) and Barros and Lettenmaier (1993) to in-fill precipitation values in remote mountainous areas using a dynamic model.

This type of simulation can be used to dynamically downscale global models for the purposes of understanding the development of weather systems and validating a forecast model's parameterisations or for defining a climatology in either a location of sparse data or under a different climate scenario or general circulation pattern. Longterm mesoscale simulations of seasonal weather were conducted by Comarazamy and González (2008), Gutowski et al. (2003), Colle et al. (2003) and (2000), Lenters (1995), and Giorgi (1990), ranging in length from a single season to a ten-year simulation. 
Downscaling techniques are often classified as dynamical or statistical. Statistical methods often require fewer resources and are faster to calculate so they are popular. For example, these were used in Anandhi et al. (2008), Hundecha and Bardossy (2008), Tait et al. (2006), Valverde Ramirez et al. (2006), Widmann et al. (2003), or Trigo and Palutikof (2001), who all conducted the downscaling for the purpose of estimating future climates under different climate change scenarios. Due to the nature of precipitation (see section 2.2), simple statistical relationships are not strictly applicable. Where the dynamics of a system are not well understood or modelled then statistical downscaling is a valuable technique.

Despite the nature of precipitation, statistical downscaling was used in Busuioc et al. (2008) to examine wintertime extreme precipitations, and Gangopadhyay et al. (2004) and Daly et al. (1994) used it for hydrological purposes. However, Gangopadhyay et al. (2004) stated that there was no valuable skill in statistically downscaling precipitation. Some of these statistical methods have become extremely sophisticated and have done a good job, for example Kioutsioukis et al. (2008) use of a 'multisite hidden Markov non-homogeneous Markov model' to downscale winter daily precipitation over Greece. Many more studies exist, some of which (e.g. Pandey et al. (2000)) have combined statistical methods with dynamic methods for downscaling. Where the resources exist and the dynamics are understood dynamical downscaling has a greater potential to provide valuable information, both in terms of accuracy and dynamical understanding.

\subsection{Model set up}

The upgraded version (2.1 to 2.2) of the WRF model used in Chapter 6 was used to dynamically downscale two consecutive years of global weather data. The WRF model upgrades did not change the physics used in the system* but the core of the system was upgraded which may affect the total volumes of precipitation generated (Gallus

*http://www.mmm.ucar.edu/wrf/users/ 
and Bresch, 2006). Version 3 of the WRF model was released in early 2009.

The initialisation data were changed to the final analysis data set (FNL), also available from NCEP. The final analysis is similar to the analysis files in the GFS data used in Chapter 6, but it is not released operationally so additional data can be included in the data assimilation. Instead of just using three hours of data prior to the analysis time, the FNL also considers data collected in the three hours afterwards. Additional remote data that are not available in real time can also be added. The extra data should make the analysis fields even more representative of the atmospheric conditions at each time step.

In addition to changing the global data, the domain and some model options were changed from the Control simulation. The changes were implemented to reduce the run-time and problems identified in Chapter 6, and are now consistent with operational forecasting resolutions. A summary of the settings is provided in Table 7.1, and the

Table 7.1

The WRF settings for the two-year dynamic downscaling simulation.

\begin{tabular}{|l|l|}
\hline Parameter & Setting \\
\hline \hline Initialisation and forcing & FNL six-hourly 1 degree model analysis \\
\hline Vertical levels & 27 \\
\hline Grid spacing & 36,12 \\
\hline Core & Advanced mass dynamical core \\
\hline Microphysics & Thompson \\
\hline Cumulus physics & Kain-Fritsch \\
\hline Topography & Standard from WPS scheme \\
\hline Surface wetness & full soil model \\
\hline Nesting & one way \\
\hline Nudging & to FNL every 6 hours \\
\hline
\end{tabular}

full namelist.input file is included in Appendix C. The model physics have been kept the same for this downscaling as during the Control simulations in Chapter 6 .

This simulation uses one-way instead of two-way nesting; this means that the inner domain does not influence the development of weather in the outer domain. To prevent 
the inner section of the outer domain from developing errors that could be propagated, the outer domain was 'nudged' (adjusted) to the FNL data every six hours. Qian et al. (2003) stated that frequent re-initialisation of a long-term simulation will result in smaller errors as it can compensate for systematic error, e.g. poor representation of moisture process in mountainous areas. However, Lo et al. (2009) have shown that full three-dimensional nudging is even more skilful, and that is what is used in this simulation. This means that the data at each degree point within the outer domain were nudged towards the FNL value every six hours if they were deviating. Nudging has been an accepted technique in data assimilation and forecasting for over 30 years (Stauffer and Seaman, 1989; Stauffer et al., 1990). As this model was being run for a continuous two-year period, nudging was required to prevent errors from compounding over the period.

Only two domains were used both covered the whole of New Zealand for verification purposes, so the data-set could later be applied to other projects. The two domain boundaries are shown in Figure 7.1, and have 36- and 12-km grids. The 12-km grid is

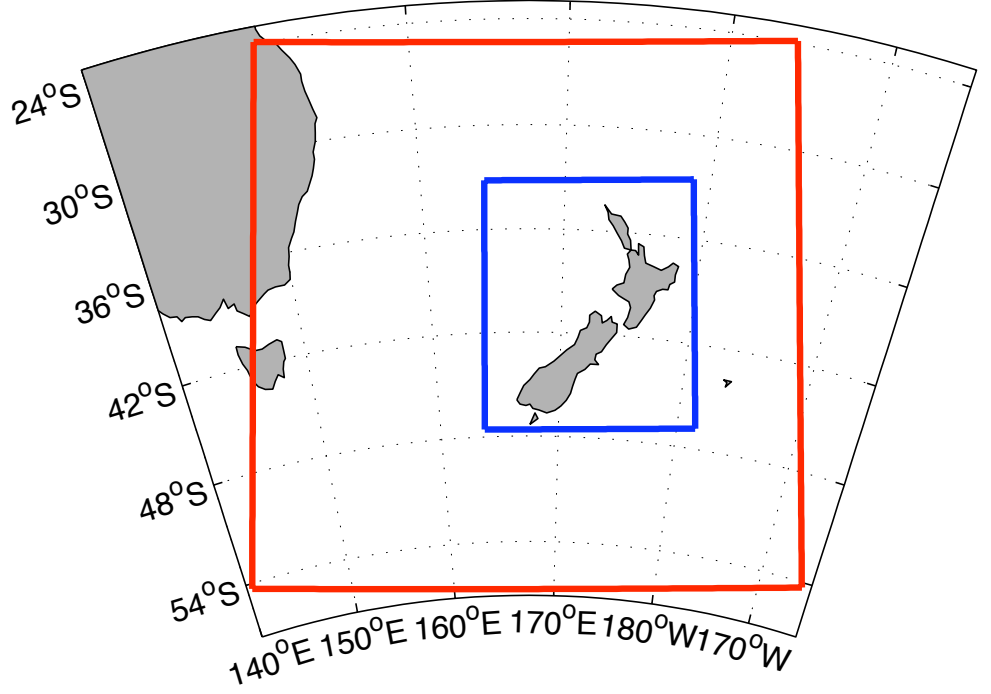

Figure 7.1 Domain boundaries for the WRF dynamic downscaling simulation.

equivalent to current operational forecasts in New Zealand and similar to the middle domain of the high-resolution simulations. The domain area was also extended to reduce boundary effects such as those seen in the 'Boundary' simulation in Chapter 6.

The two-year simulation can be used to calculate a precipitation climatology for 
each of the subcatchments along the Waikato River, which will provide greater detail of the climate for the purposes of managing the river. The grid squares that represent each of the subcatchments are shown in Figure 7.2. This shows that each of the

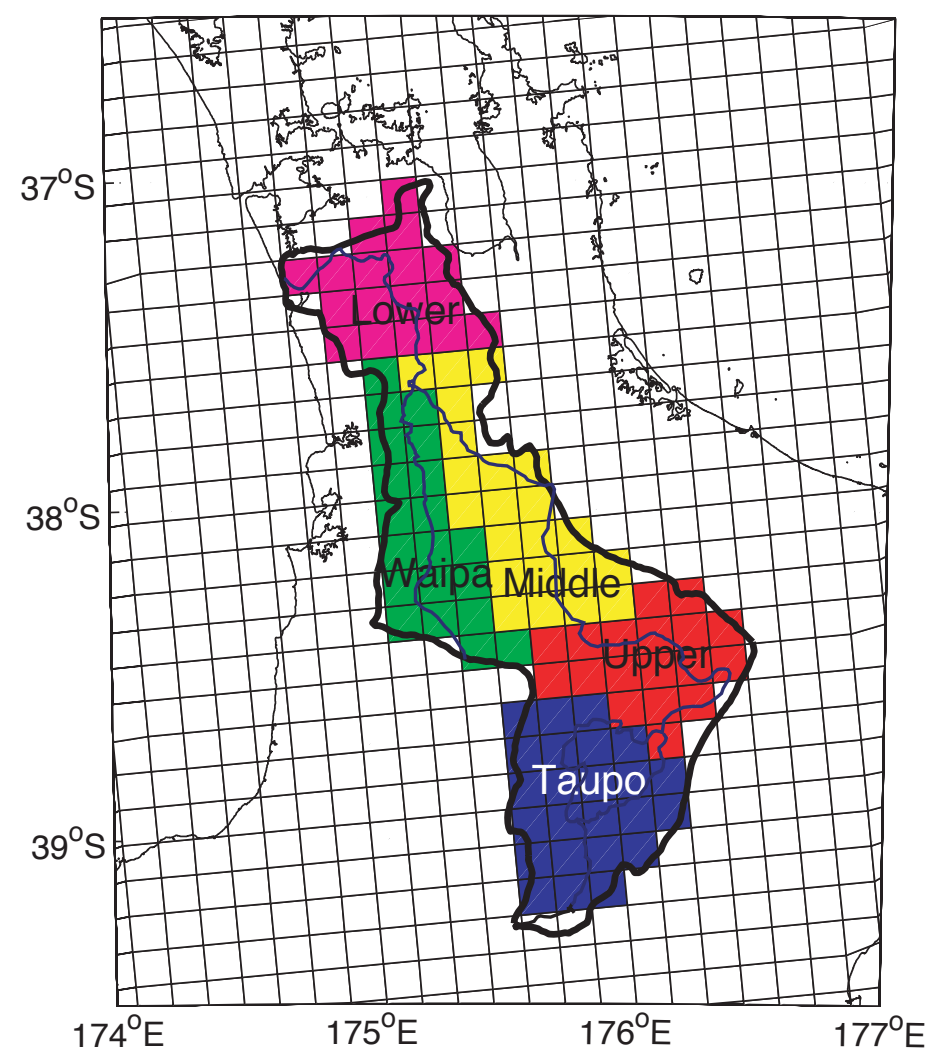

Figure $\quad 7.2$ The inner domain grid of the dynamic downscaling simulation showing which grid squares are in each subcatchment.

Waikato River subcatchments is represented by multiple grid squares in this downscaling simulation.

\subsection{Data comparison}

The downscaling simulation is first validated against existing observational data before being used to infer climate in data-sparse locations. For this comparison the total precipitation (observed and simulated) over the calendar year 2006 was accumulated at 644 rain gauge locations throughout New Zealand (Figure 7.3). Observed precipitation accumulations reached $10,000 \mathrm{~mm}$ along the west coast of the South Island. In the Waikato region the maximum accumulations were half of this amount and therefore this area has been enlarged in Figure 7.4. Validation has been conducted over all of 

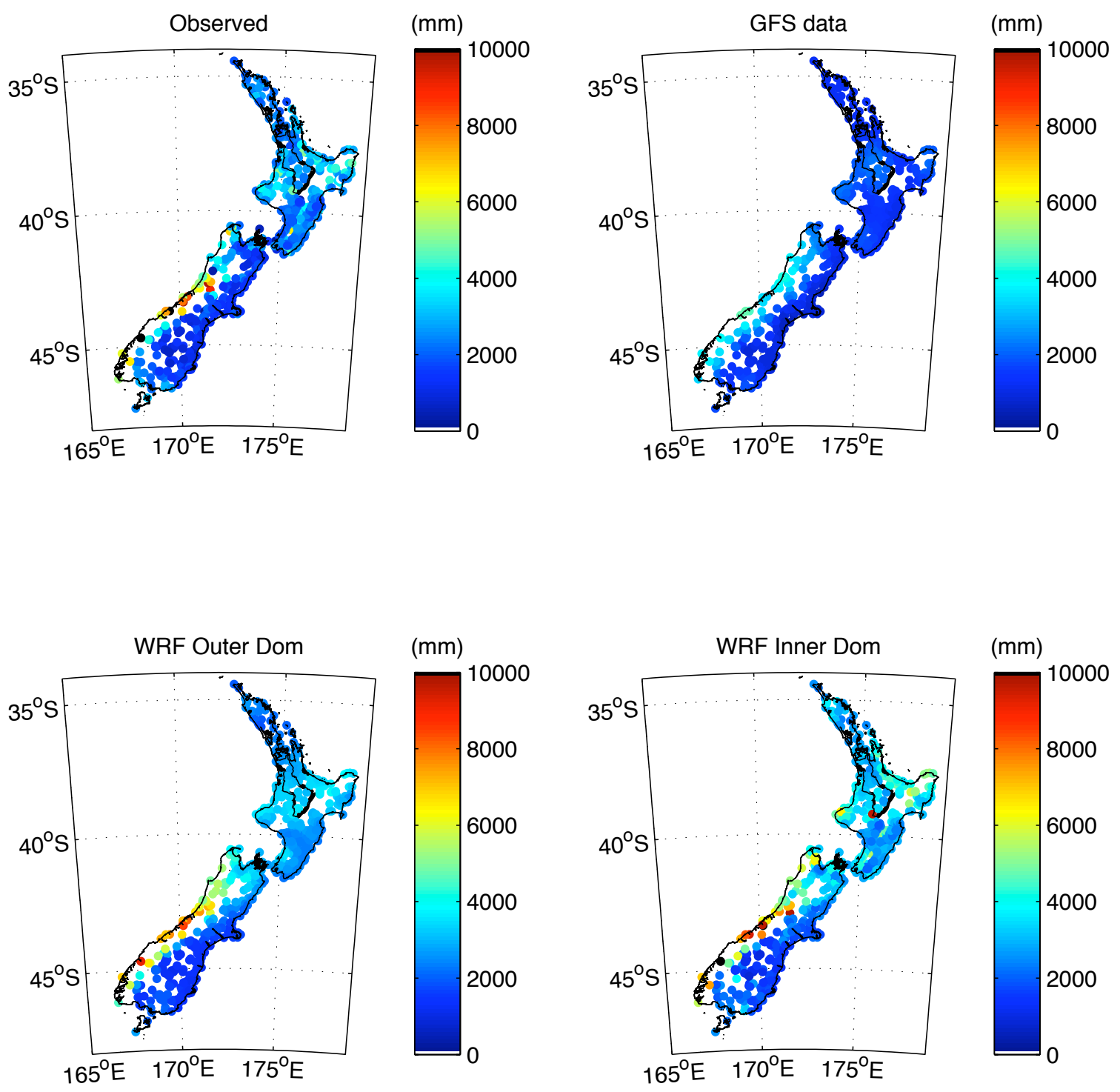

Figure 7.3 Comparison of the 2006 total accumulated precipitation for New Zealand; (a) observed values at each gauge station, (b) values interpolated from the GFS six-hour lag forecasts at each gauge station, (c) the outer domain of the dynamic downscaling simulation interpolated to each gauge location, and (d) the inner domain of the dynamic downscaling simulation interpolated to each gauge location.

New Zealand to show the general skill of the model as many of the events that effect the Waikato River catchment also have an impact on other locations in New Zealand.

The FNL data used to initialise this model simulation contain no precipitation data and therefore the six-hour lag forecasts from the GFS model have been used in the above figures to approximate the global model simulation of precipitation during this period. In both Figures 7.3 and 7.4 the global model produced a smooth precipitation 

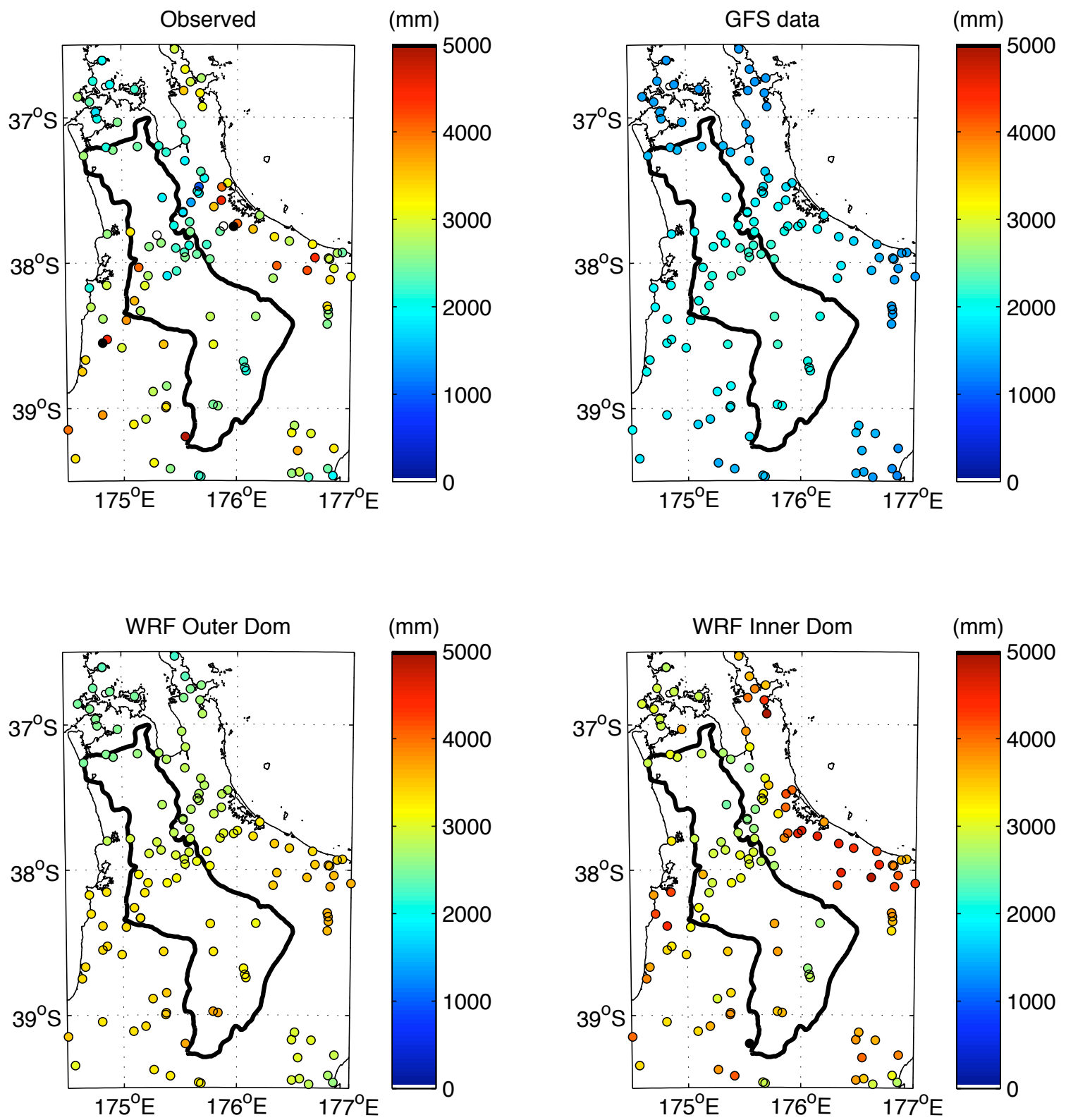

Figure 7.4 Comparison of the 2006 total accumulated precipitation for the Waikato; (a) observed values at each gauge station, (b) values interpolated from the GFS six-hour lag forecasts at each gauge station, (c) the outer domain of the dynamic downscaling simulation interpolated to each gauge location, and (d) the inner domain of the dynamic downscaling simulation interpolated to each gauge location.

distribution that substantially under-predicts the precipitation totals observed.

Although the 36-km grid significantly increases the variability and total volume of precipitation it is still unable to simulate the enhancement and sheltering provided by the topography. The higher resolution inner domain was unsurprisingly the best at 
representing the observed precipitation totals over this year-long period throughout New Zealand. This simulation has over-predicted precipitation around the coastline in many places but the distribution pattern indicates that the model has replicated the enhancement and sheltering effects of many topographical features. For example, the sheltering effect of the Kaimai Ranges is identified; and is required to estimate precipitation within the Waikato River catchment.

\subsubsection{Daily simulation comparison}

The distribution of annual precipitation totals in the inner domain of the simulation provided a good approximation to the totals observed in most parts of New Zealand. However, this aggregation did not show the temporal distribution of precipitation throughout the year. Therefore, the mean error and rmse were calculated between the daily precipitation totals (Observed and Downscaled) at each rain gauge station throughout New Zealand. The mean errors in Figure 7.5 show that for the majority of stations the mean error is approximately zero. There are negative errors in many locations where there are high rainfall totals, e.g. the west coast of the South Island, Mt Egmont/Taranaki and the Tararua Ranges. However, there are also locations with positive errors such as Mt Ruapehu or the north of the South Island. The positive errors represent an over-prediction of the model. Unlike Ibbitt et al. (2001) who had under-prediction on the west and over-prediction on the east of New Zealand's South Island, our simulation (on a updated model) now over-predicts precipitation on the west coast and has almost zero mean error on the east coast.

Figure 7.6 is an enlargement of the mean errors for the central North Island. This enlargement contains Mt Egmont/Taranaki, which had the largest negative error, and Mt Ruapehu, which had the largest positive error of the entire domain. The mean error at most stations is less than $\pm 2 \mathrm{~mm}$ indicating that at the majority of gauges there is little systematic bias in precipitation predictions. In some locations, such as near Taupo, there are two nearby gauges that have different errors indicating that there 


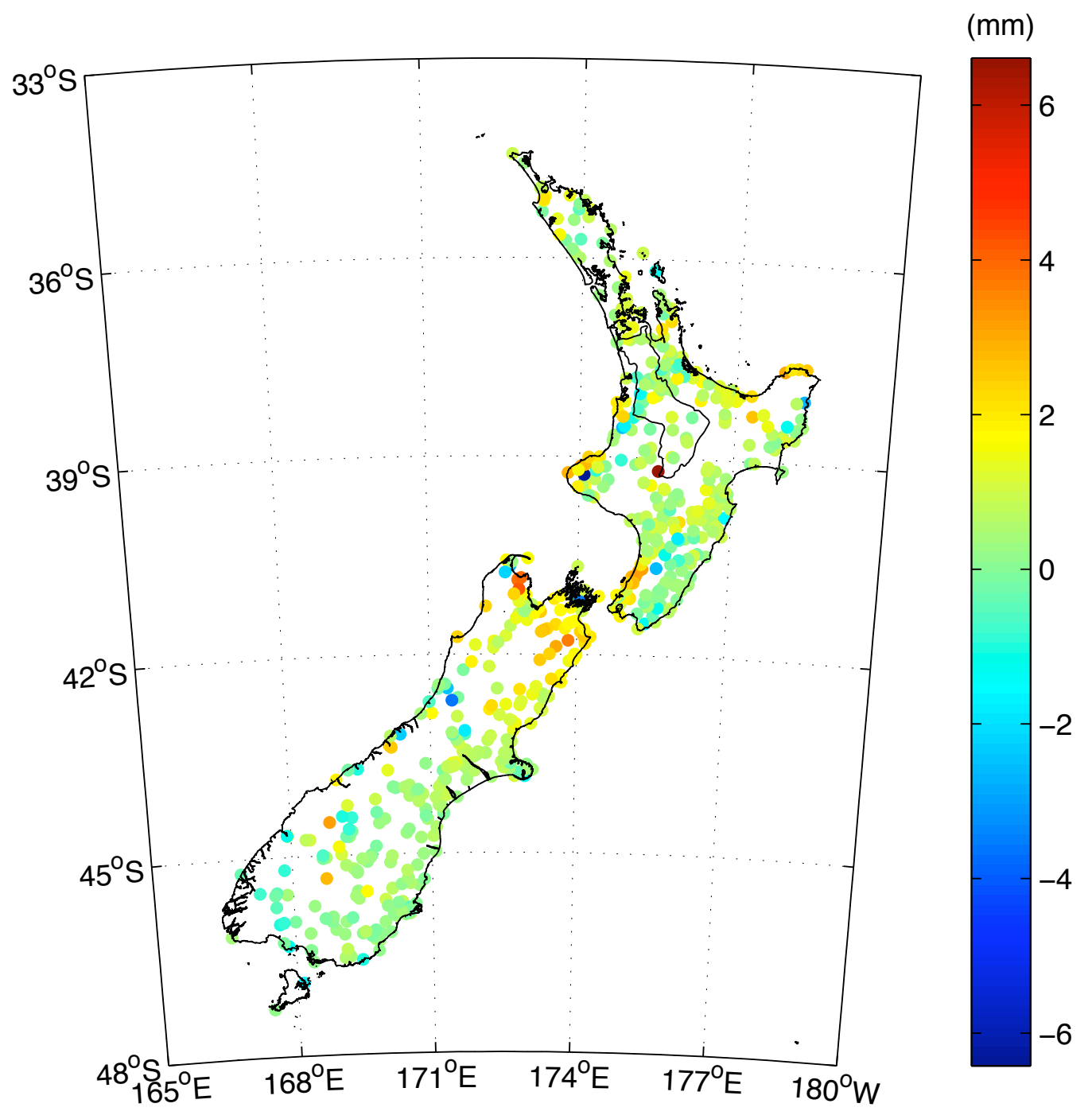

Figure 7.5 The mean error between the inner domain downscaling daily precipitation time series and observed precipitation at each of the gauges in New Zealand.

are localised effects or possible measuring errors altering the precipitation totals in at least one of these gauges. Throughout the Waikato River catchment the errors tend to be slightly positive, but are very small in magnitude. This indicates that the model was slightly over-predicting the precipitation on a daily basis within the catchment area. The small negative errors in the King Country suggests that this simulation has underestimated the enhancement effect along these hills and allowed excess moisture to spill over into the Waikato catchment.

The rmse for each station in New Zealand is shown in Figure 7.7. The same locations 


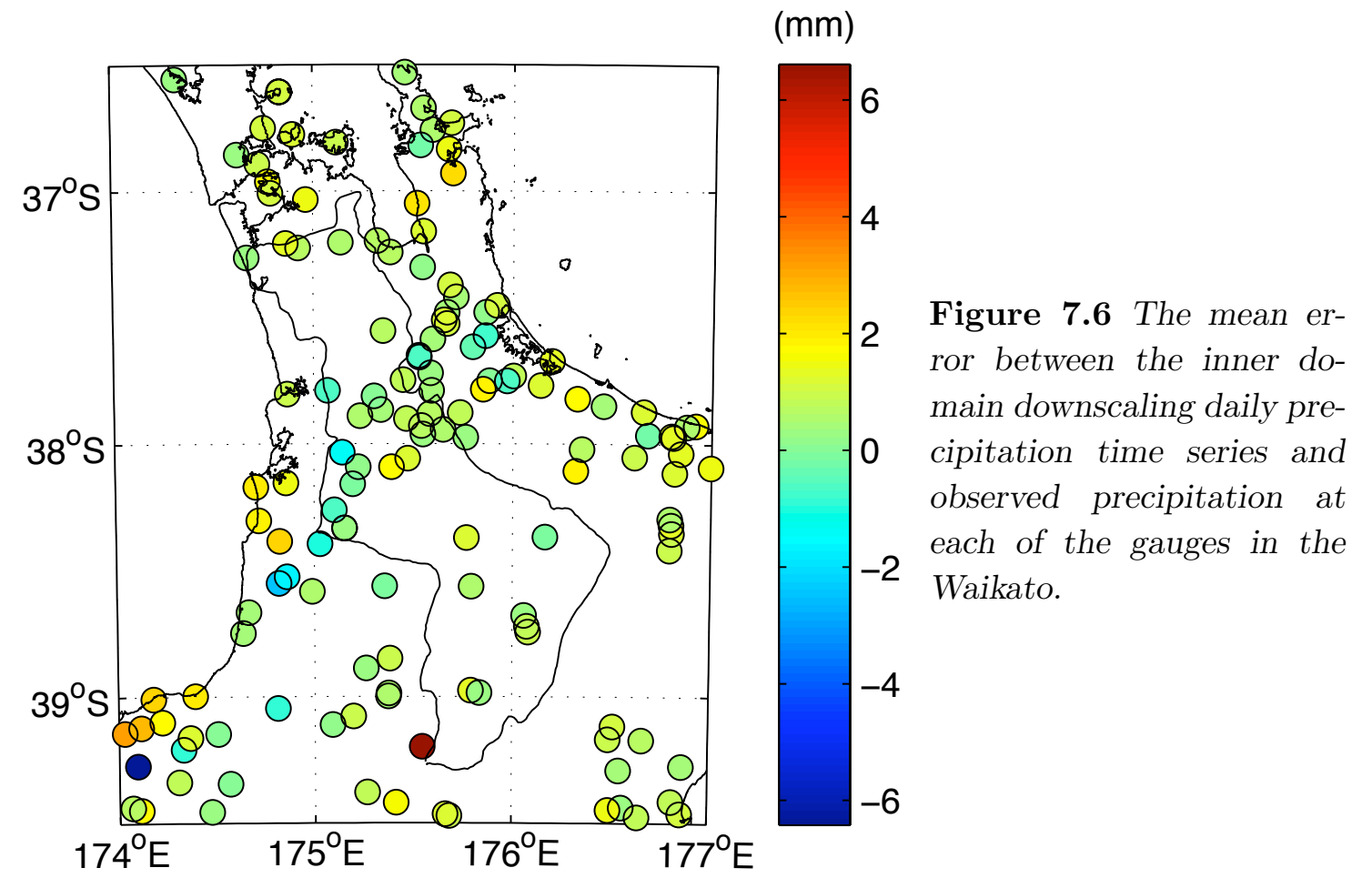

that had large magnitude mean errors (both positive and negative) have large rms errors as well. For the majority of stations the average error will be less than $5 \mathrm{~mm}$ of precipitation on any given day, although in the central North Island (Figure 7.8) the average errors are closer to $10 \mathrm{~mm}$ on any given day. The largest error is at $\mathrm{Mt}$ Egmont/Taranaki where the rmse is in excess of $30 \mathrm{~mm}$. The rmse was plotted as a function of mean error for all 644 stations in Figure 7.9. As expected the largest mean errors where recorded at stations that also had large RMSE values, and contain more complex topography. However there are also stations with a small magnitude mean error that still has RMSE values in excess of $20 \mathrm{~mm}$, indicating that these locations do not contain a systematic bias in the model.

The mean absolute percent error was also calculated for the stations around New Zealand. Figure 7.10 shows the mape at each of the stations that had a long enough record to calculate this error. The mape clearly show a latitudinal variation throughout New Zealand with larger map errors in the north and smaller errors in the south with the exception of a few locations such as the Canterbury plains that have relatively high map errors. The enlargement of the Waikato River catchment in Figure 7.11 


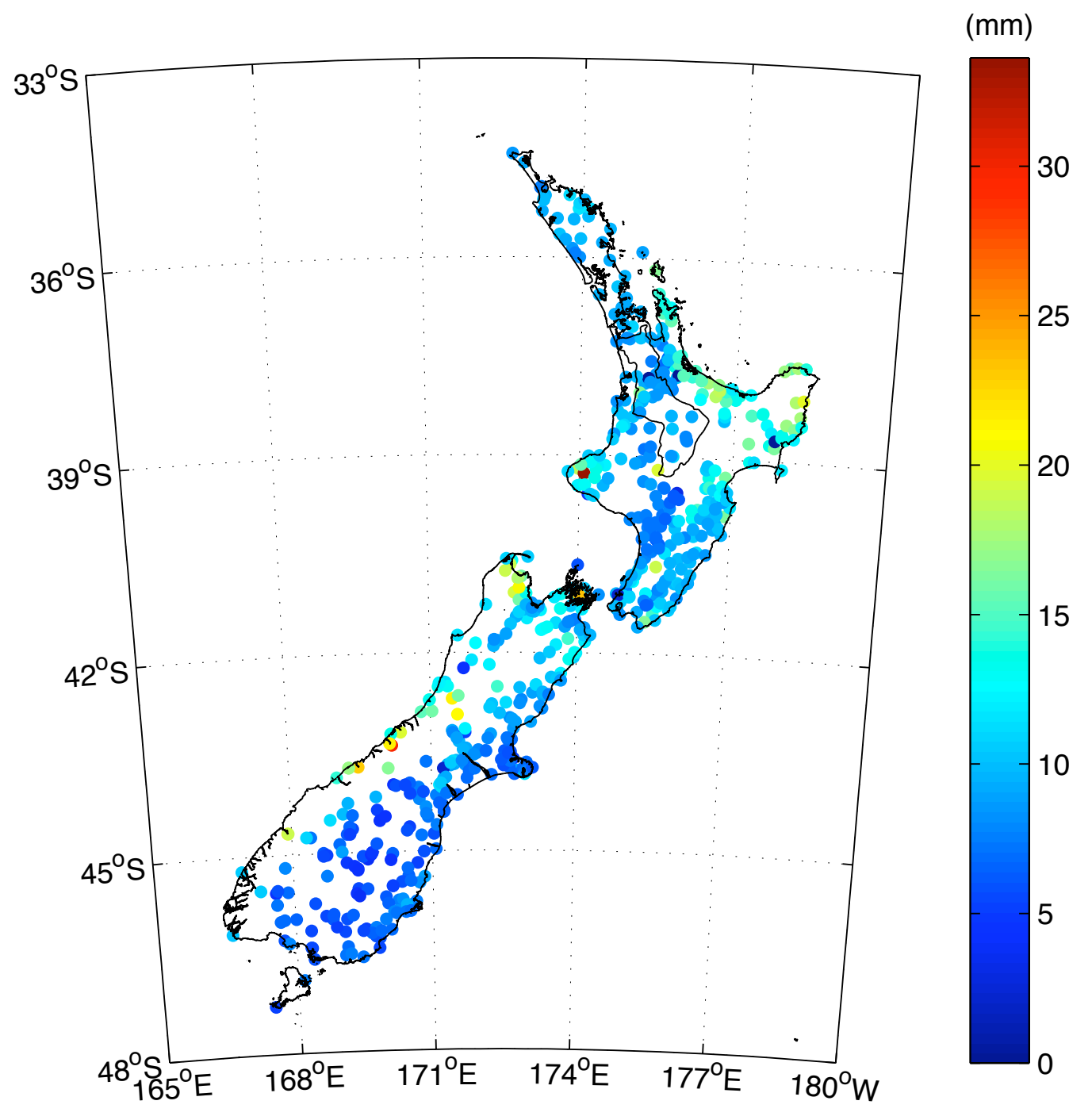

Figure 7.7 The rmse between the inner domain downscaling daily precipitation time series and observed precipitation at each of the gauges in New Zealand.

shows that there is considerable variation around the catchment. The inland locations generally have smaller map errors with larger errors in the Waipa subcatchment and near the mouth of the river. In both of these maps the mape are between six and $15 \%$ indicating that the model is generally producing realistic precipitation values.

Figure 7.9 clearly shows that most stations have a mean error between 0 and 2 indicating that the model has a tendency to over-predict precipitation throughout NewZ̃ealand. Figure 7.9 also shows that the gauges within the catchment boundary all have small, slightly positive mean errors. This contradicts the general under-prediction 
(mm)

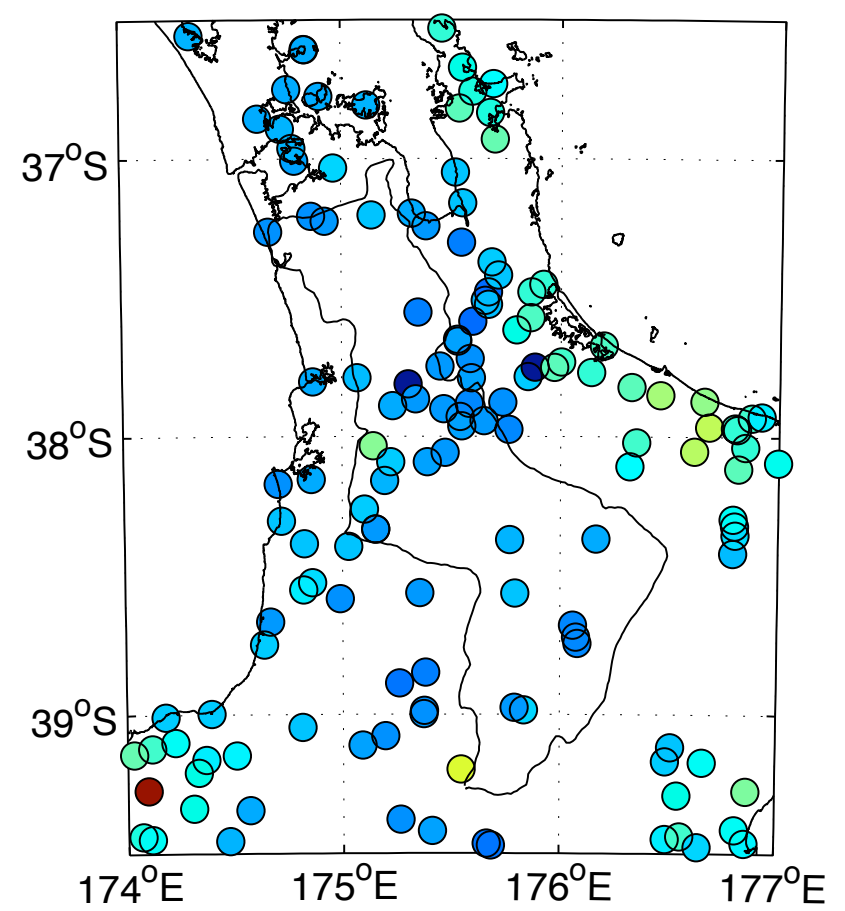

Figure 7.8 The rmse error between the inner domain downscaling daily precipitation time series and 15 observed precipitation at each of the gauges in the 10 Waikato.

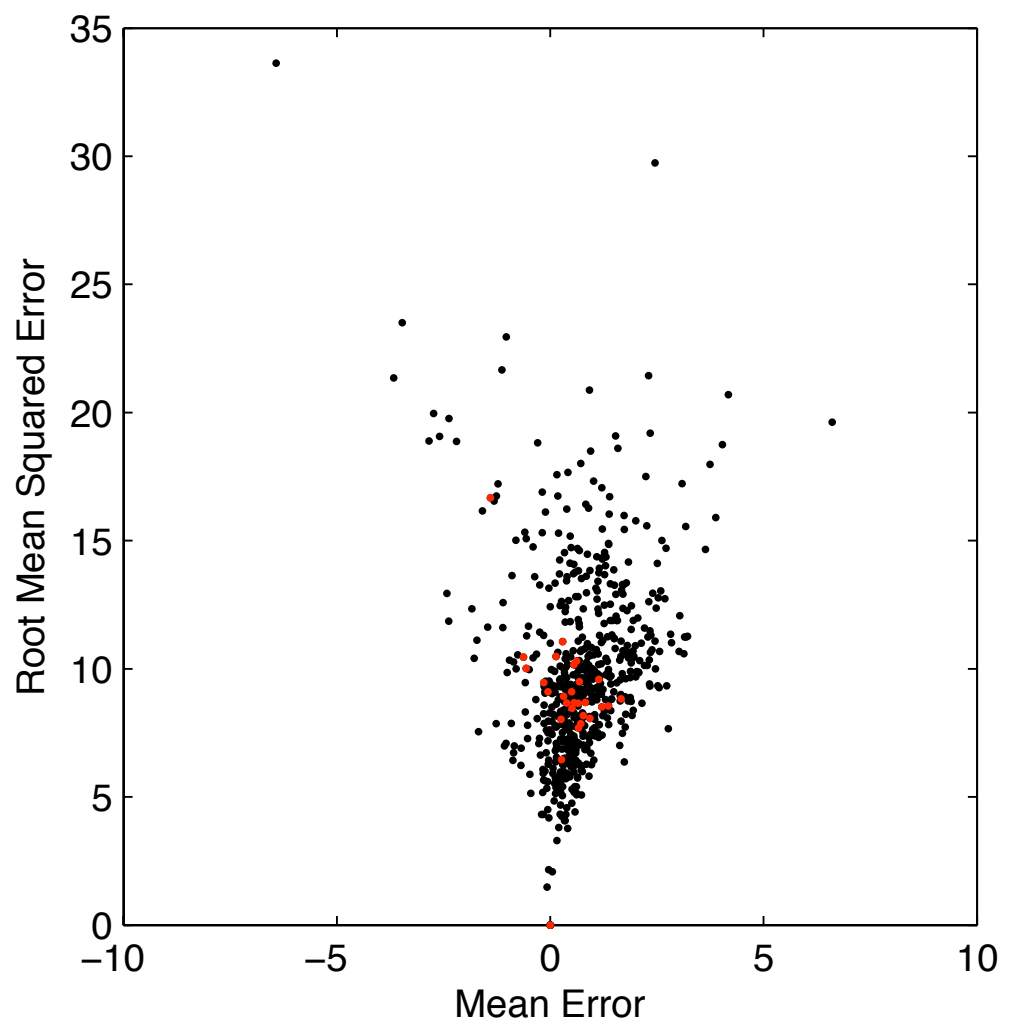

Figure 7.9 Comparing mean error and rmse at the 644 gauge locations throughout New Zealand in black, and for the stations within the Waikato River catchment in red. 


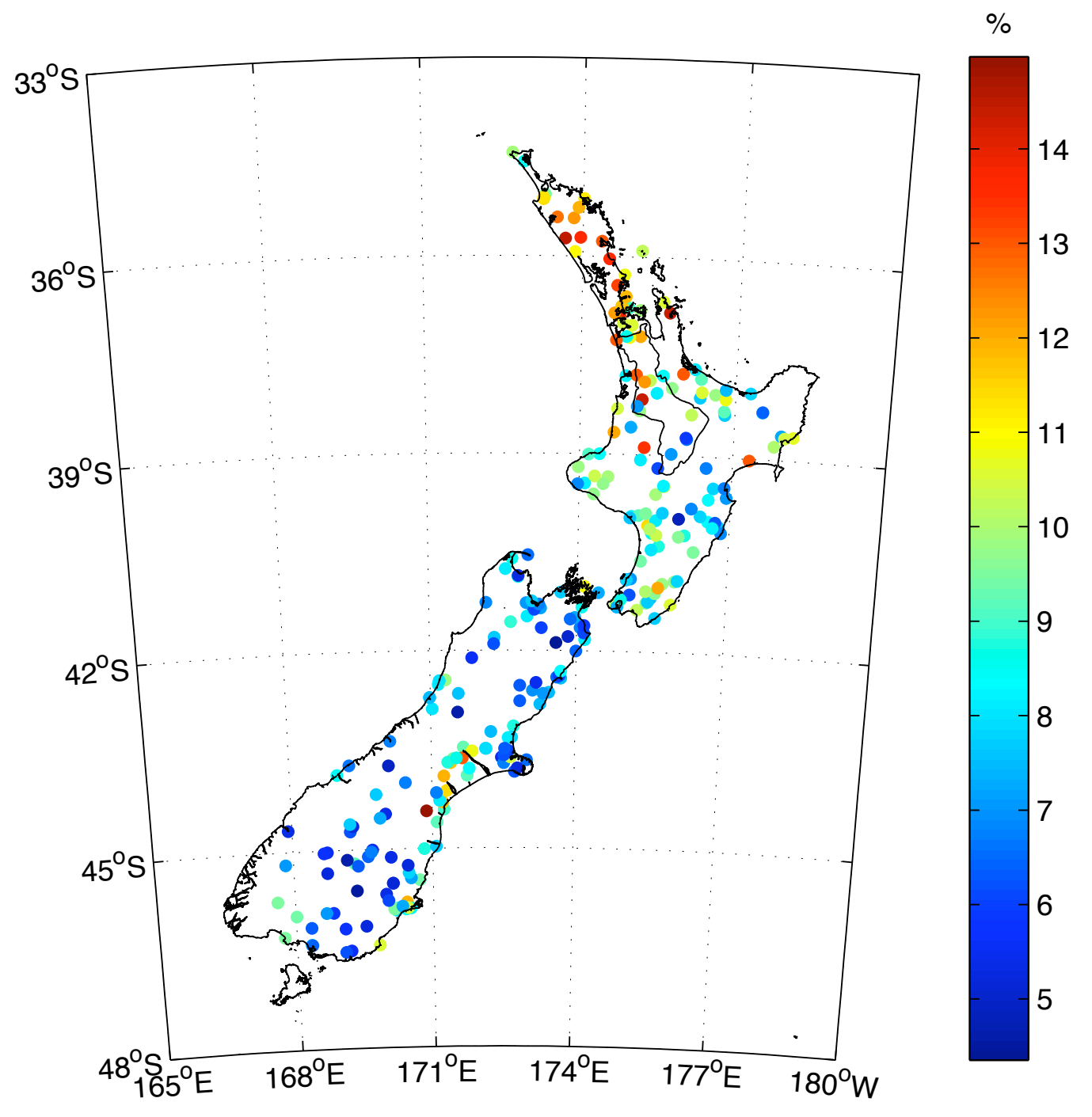

Figure 7.10 The mean absolute percentage error between the inner domain downscaling daily precipitation time series and observed precipitation at each of the gauges in New Zealand.

seen in Chapter 6 but could be due to either the upgrade of the model core or a preference to over-predict light precipitation and under-predict heavy precipitation (like the GFS model in Chapter 5).

\subsubsection{Case study comparison}

Chapter 6 showed that small differences in the model set-up could create differences in the total amount of precipitation generated by the model. To relate the results of the high-resolution simulations to this dynamic downscaled data, the hourly time series 


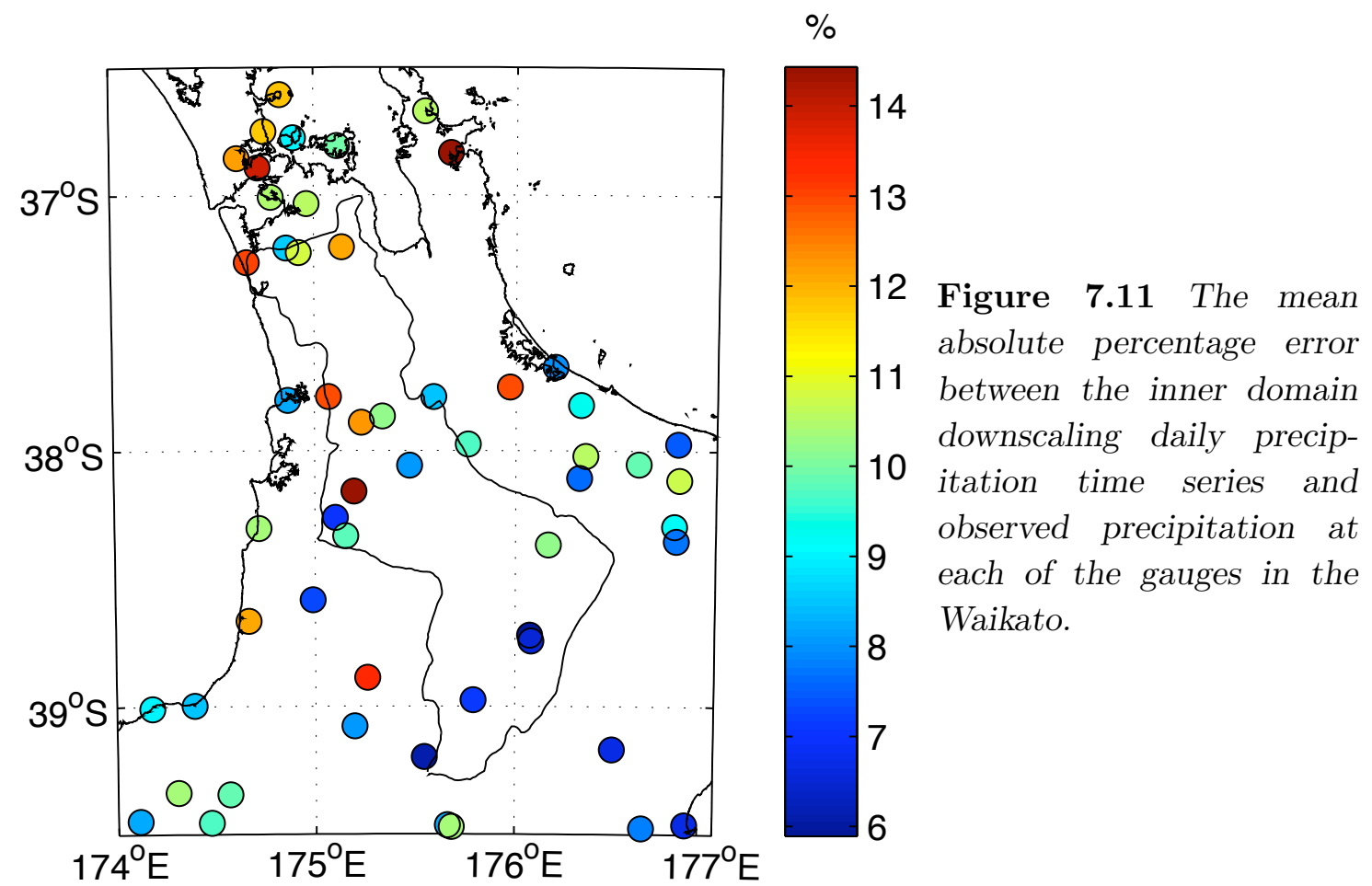

from both models are compared to the real data at each of the six observation stations shown in Figure 6.5 for each of the three cases studied in Chapter 6.

As described earlier, October 2005 (Figure 7.12) saw unstable tropical air entrained between two mid-latitude cyclones resulting in heavy showers and thunderstorms over the period of a week. All three time series (Observed, Control, and Downscaled) show multiple periods of precipitation over the event. At the northern stations, 2006 and 1547, the downscaling simulation produced more precipitation than was modelled by the high-resolution simulation and at station 1547 the downscaling simulation generated excessive precipitation compared to observations. The general shape of the time series is observed and the downscaling predicted $110 \%$ of the observed precipitation within the Waikato catchment, whereas the high-resolution simulation predicted only $92 \%$ of the total precipitation.

A subtropical cyclone and the front associated with a Southern Ocean cyclone were stalled over New Zealand by a blocking high in January 2006. This event had distinct frontal and convective precipitation periods that have been shown in detail in Chapters 
Oct 2005

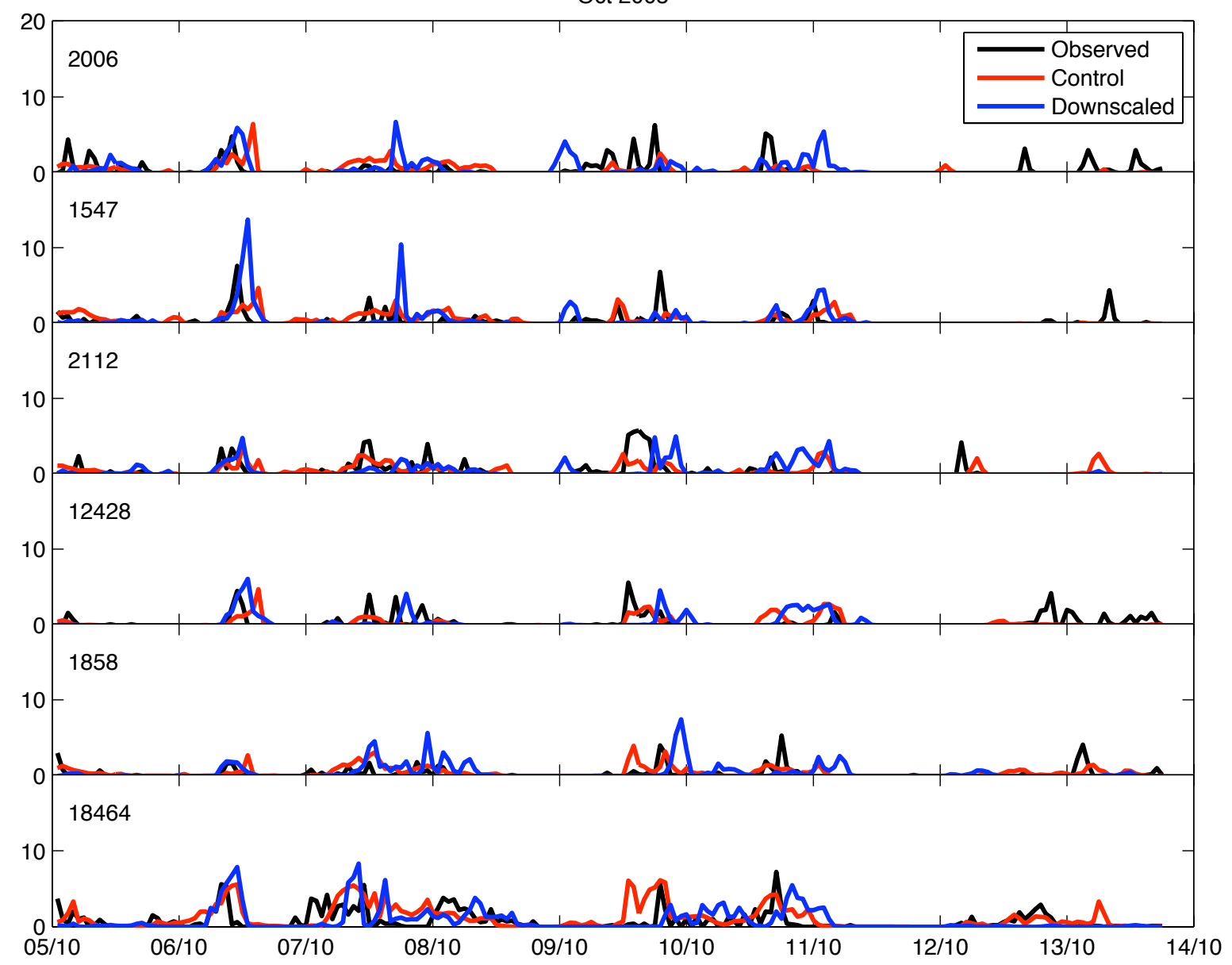

Figure 7.12 Hourly precipitation observations and time series from the dynamical downscaling, and high-resolution simulations for the event of October 2005.

4 and 6. The downscaled data (Figure 7.13) did not separate the distinct precipitation periods seen in Chapter 6, suggesting that in this simulation the two features arrived simultaneously. The downscaling domain boundary was also extended much further than the high-resolution domain as this was expected to reduce the effects of the domain boundary on the timing of the subtropical cyclone (as discussed in section 6.3.4). However, this increased boundary has delayed the arrival of the subtropical cyclone even more and advanced the arrival of the cold front to create a single precipitation period. The dynamic downscaling only managed to produce $61 \%$ of the total observed precipitation during this event, compared to the $83 \%$ in the high-resolution simulations.

The downscaled simulation of the October 2006 event (Figure 7.14) still failed to 


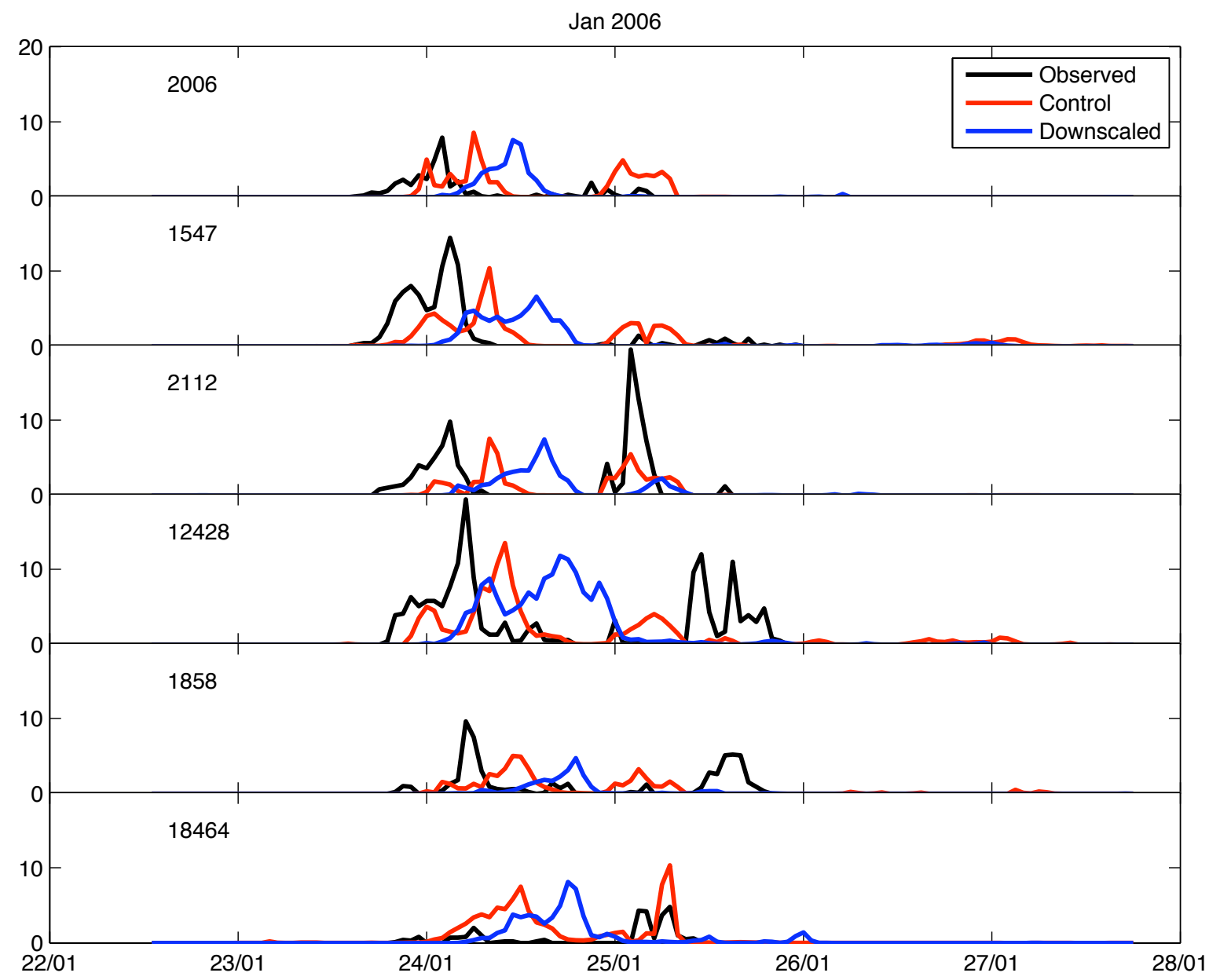

Figure 7.13 Hourly precipitation observations and time series from the dynamical downscaling, and high-resolution simulations for the event of January 2006.

simulate the first precipitation period of this event on 1 October; initially this was attributed to being too close to the start of the model run in the high-resolution simulations and being missed in the model spin-up. However, model spin-up cannot account for why the dynamic downscaling also missed this peak. The downscaling simulation generated a significant precipitation peak 20 hours later at station 1547 and another peak at station 18464 another 12 hours later again. Neither of these intense precipitation periods was observed. This event contained significant convective precipitation and, as seen in Chapter 6, this precipitation was not evenly distributed or physically constrained; meaning that the difference between these time series does not necessarily reflect the quality of the model. The total volume of precipitation 


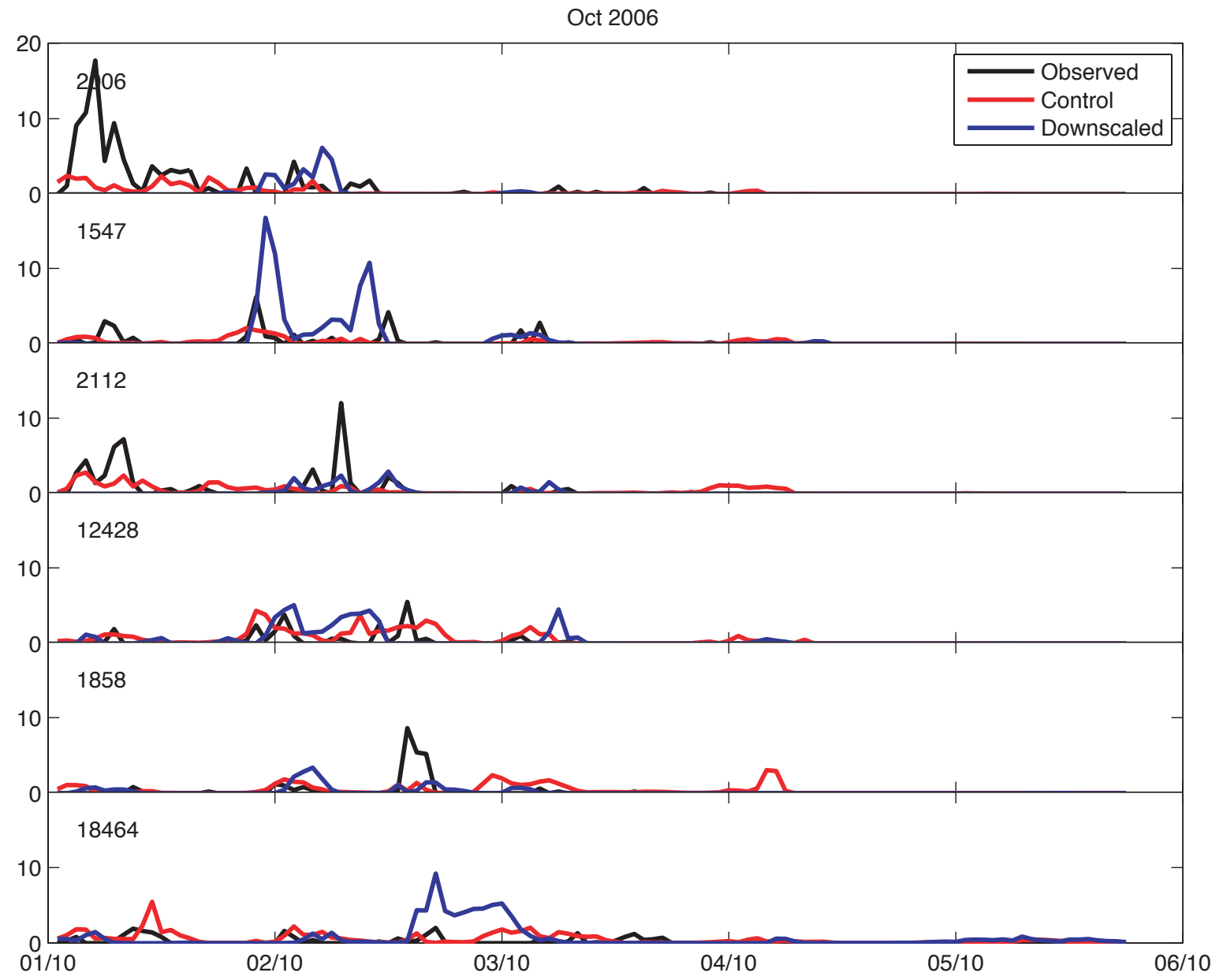

Figure 7.14 Hourly precipitation observations and time series from the dynamical downscaling, and high-resolution simulations for the event of October 2006.

predicted during this period over the whole catchment was $130 \%$ of the total observed precipitation compared to the $78 \%$ in the high-resolution simulations.

\subsection{Climatology}

The WRF mesoscale model upgrade between the high-resolution simulations and the downscaling simulation have led to an increase in the amount of convective precipitation generated, thereby increasing the total precipitation. The reduction in resolution has reduced the enhancement effect of the King Country meaning the precipitation totals simulated within the Waikato River catchment are slightly higher than observed. 
Similar to Giorgi et al. (1993), the general distribution of precipitation was representative and the precipitation totals were realistic for most, and it is considered appropriate to approximate the climate from this downscaling simulation.

Two years is insufficient to calculate a statistically significant climatology, but averaging over the two years should approximate the climate. Both of these years were warm and dry in general but neither exhibited extreme climate patterns ${ }^{\dagger}$. The average total annual precipitation for New Zealand (Figure 7.15) shows that the largest volumes of precipitation are over the Southern Alps and large topographical features in the North Island, as expected.

Convective precipitation is extremely difficult to predict or model as it cannot be physically constrained. Determining the proportion of the precipitation that was generated through the cumulus parameterisation (Figure 7.15b) should estimate the amount of convection that occurs in each area. The northern third of the domain has a high proportion of convective precipitation. Along the western coastlines, there is a high proportion of convective precipitation, which is likely driven from the land/sea temperature contrast and up-slope vertical motion when air is pushed inland. These are also areas of high total precipitation and they tended to be slightly over-estimated in the data comparison (Figures 7.7 and 7.8). This could imply that the increases in total precipitation in the upgraded model are due to an increase of convective precipitation.

In the central North Island (Figure 7.16) the minimum annual precipitation total was modelled as near 2,000 mm that coincides with values calculated in Chapter 3 . Regions known for high precipitation values, such as Mt Ruapehu, Mt Egmont/Taranaki and the Bay of Plenty all show higher precipitation values than surrounding areas. Chapter 4 identified that most heavy precipitation is associated with the passage of mid-latitude cyclones passing through the Tasman Sea so it is realistic for the simulation to have produced higher precipitation totals along the western coast.

Convective precipitation is again more prominent along the coastlines, in Lake

${ }^{\dagger}$ New Zealand National Climate Summary: http://www.niwa.cri.nz/ncc/cs/annual/aclimsum_07 


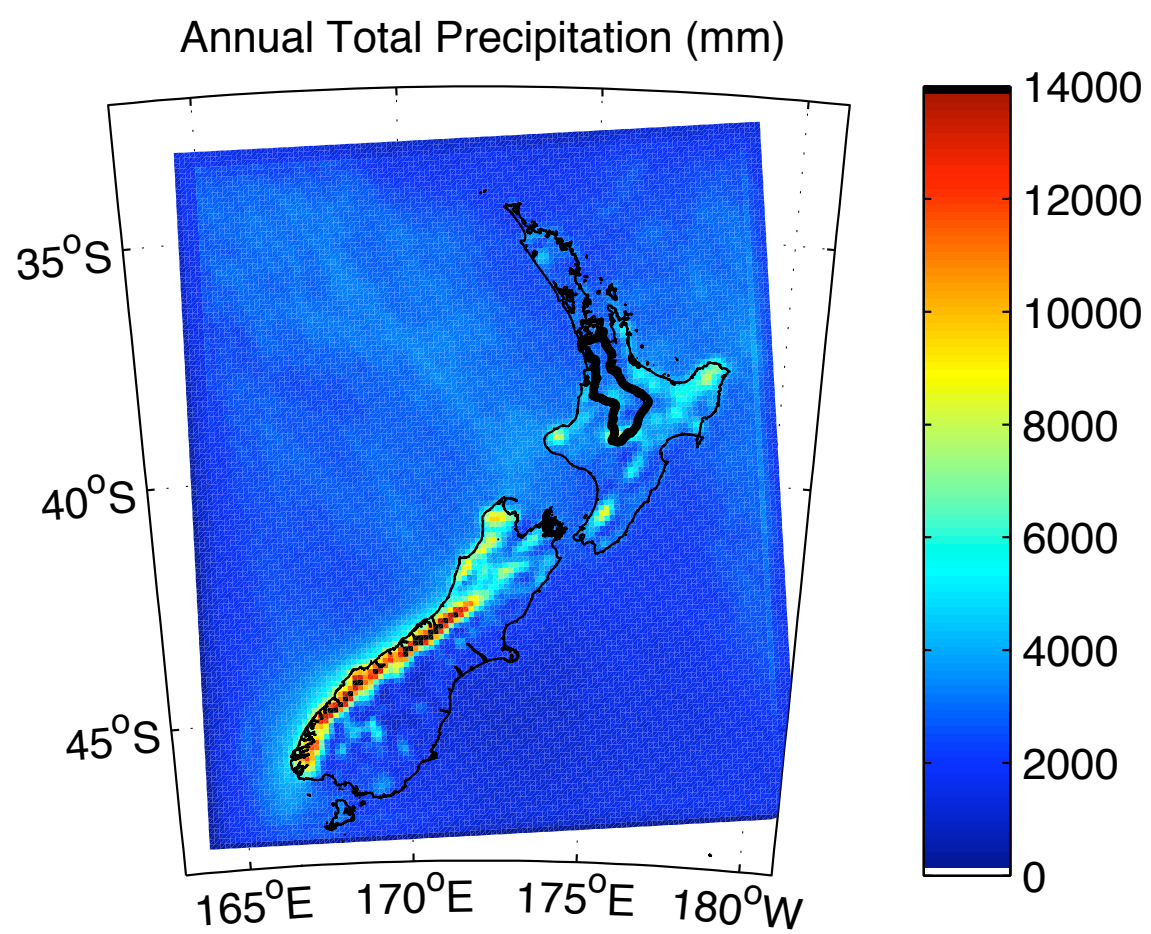

\section{Annual Proportion Convection (\%)}

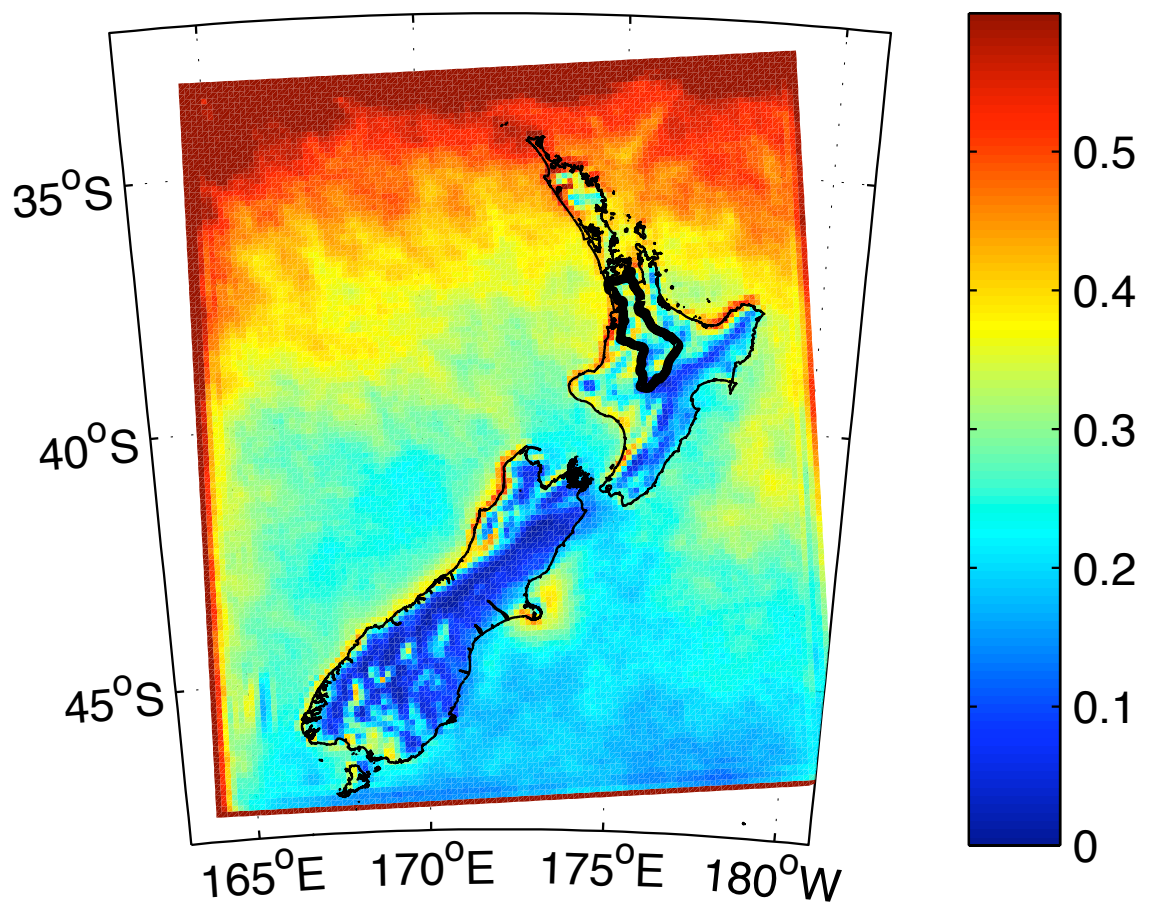

Figure 7.15 (a) Average 2005-2006 total annual precipitation in New Zealand according to the downscaling simulation, and (b) the proportion of the precipitation that was generated through cumulus parameterisation. 

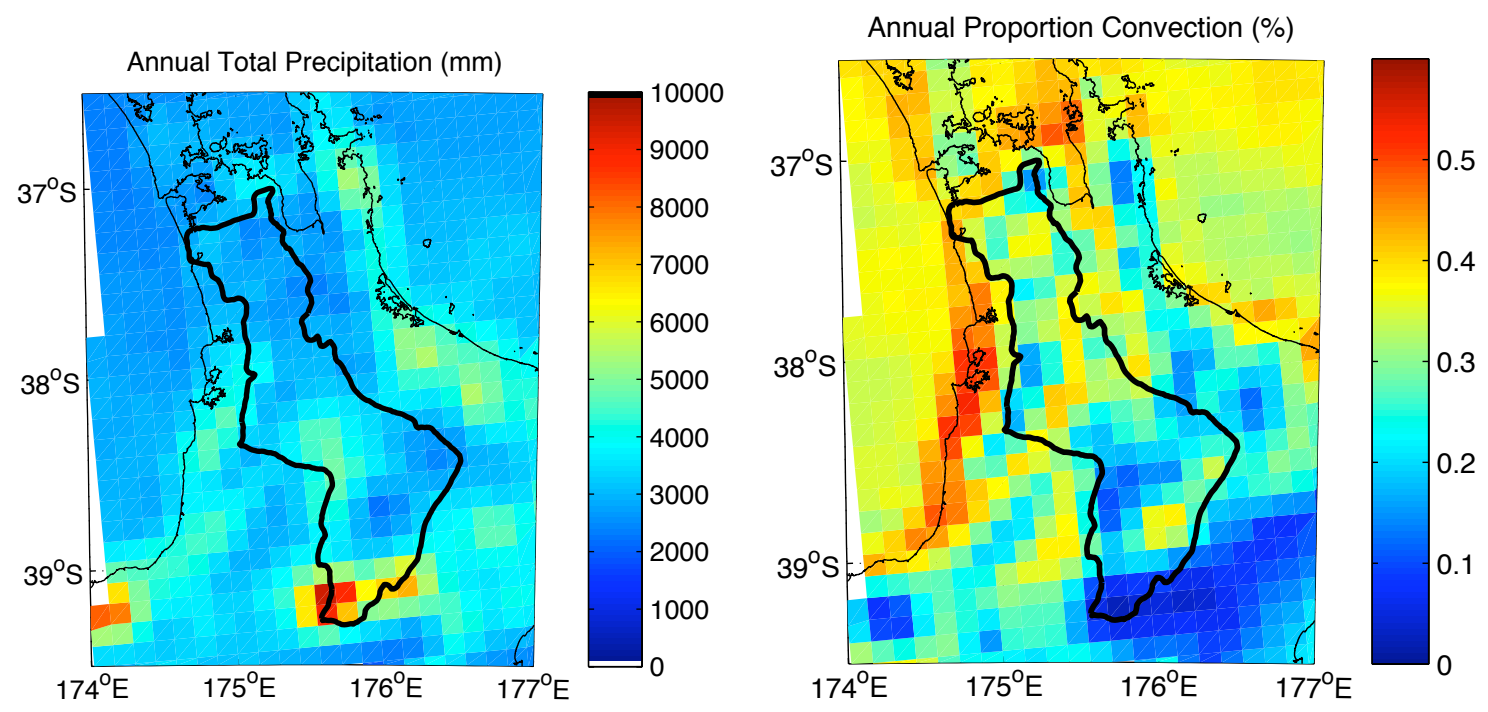

Figure 7.16 (a) Average 2005-2006 total annual precipitation in the Waikato according to the downscaling simulation, and (b) the proportion of the precipitation that was generated through cumulus parameterisation.

Taupo and in the Waipa and lower Waikato River subcatchments that are uncontrolled. The land/water surface temperature difference at Lake Taupo seems to produce more convective precipitation over the lake. At the head of the Waikato river on Mt Ruapehu there is very little convective precipitation. There is also a region east of Mt Ruapehu that contains very little convective precipitation despite both areas having high total precipitations exceeding 5,000 $\mathrm{mm}$ per year in this area. This indicates that at these higher altitude locations the model does not produce as much convection.

The Waikato River catchment has distinct seasons, so a seasonal climatology was calculated. Summer is traditionally the warmest and driest part of the year, but as was discussed in Chapter 4 some of the largest flooding events have occurred during this season. As expected, the largest precipitation total in summer is seen near Mt Ruapehu (Figure 7.17). There is also higher precipitation along the southern Kaimai Ranges and along the west coast, which is consistent with the more northerly approach of cyclones during this month (see Chapter 4). Very little precipitation was generated in the north and in the Lower Waikato subcatchment. A larger proportion of the precipitation generated in the Upper and Middle subcatchments were convective in 

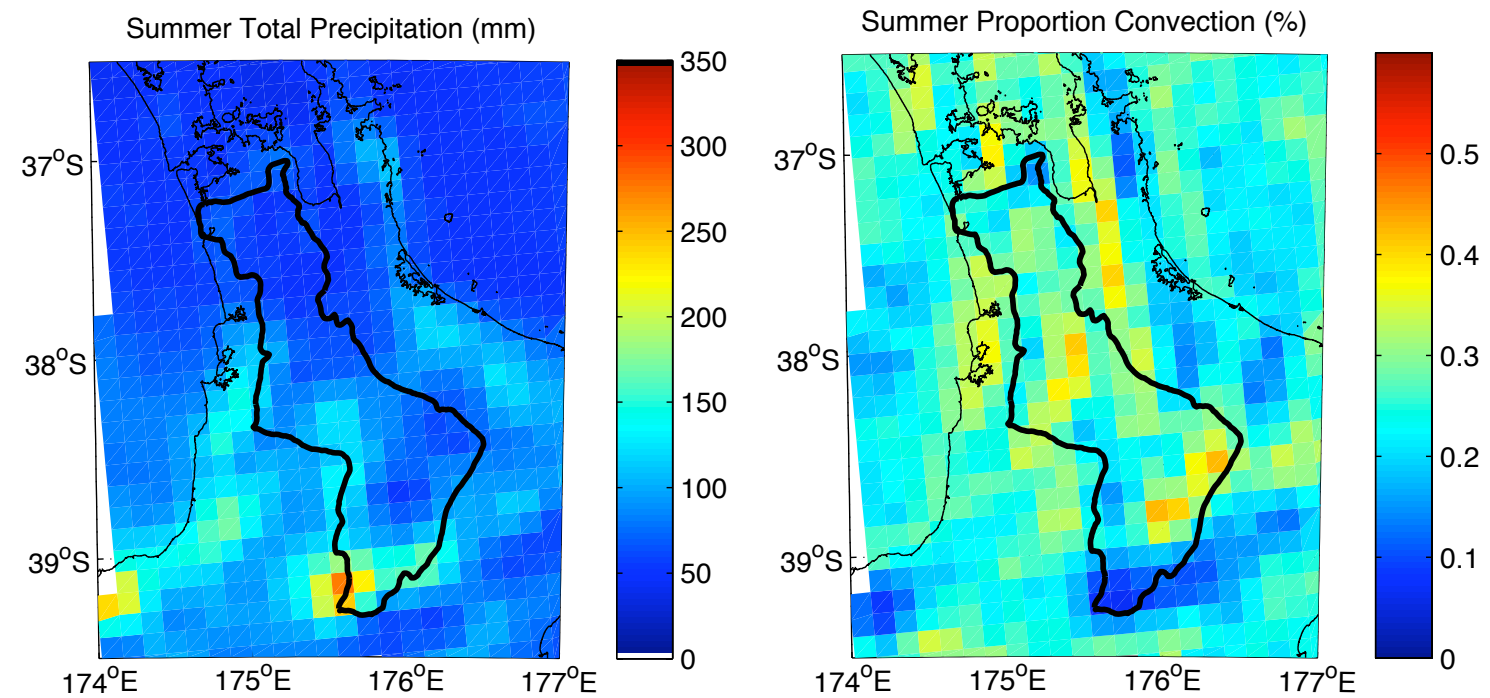

Figure 7.17 (a) Average total summer precipitation in the Waikato according to the downscaling simulation, and (b) the proportion of precipitation that was generated through cumulus parameterisation.

summer.

More precipitation was generated during Autumn (Figure 7.18), particularly con-
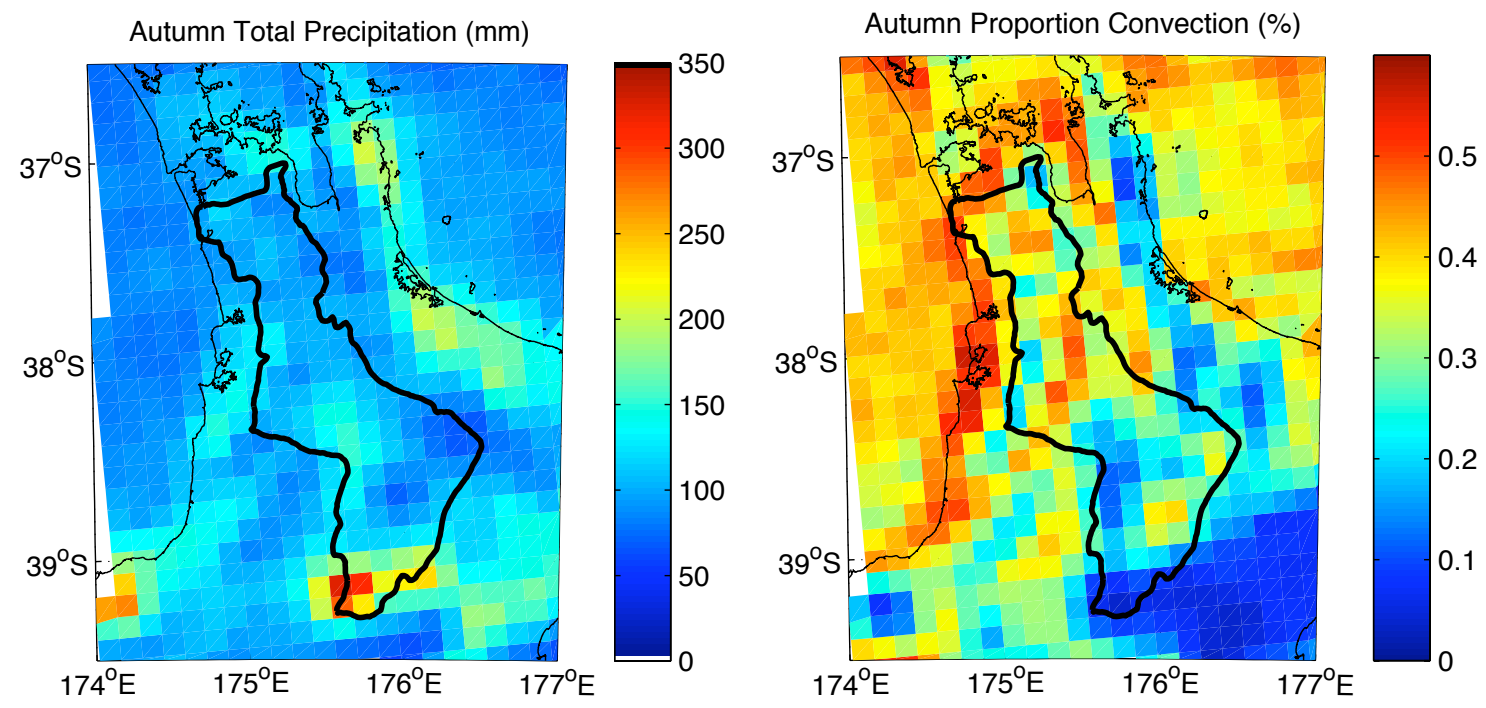

Figure 7.18 (a) Average total autumn precipitation in the Waikato according to the downscaling simulation, and (b) the proportion of the precipitation that was generated through cumulus parameterisation.

vective precipitation throughout the shown domain with the exception of the southeast corner. This coincides with an observed increased in thunderstorms during Autumn. In 
the Taupo and Upper Waikato subcatchments there is little difference in the proportion of the precipitation that is convection-related.

Precipitation totals were larger in winter (Figure 7.19, note that the scale of this figure is different to Figures 7.17 and 7.18). However, the distribution of precipitation
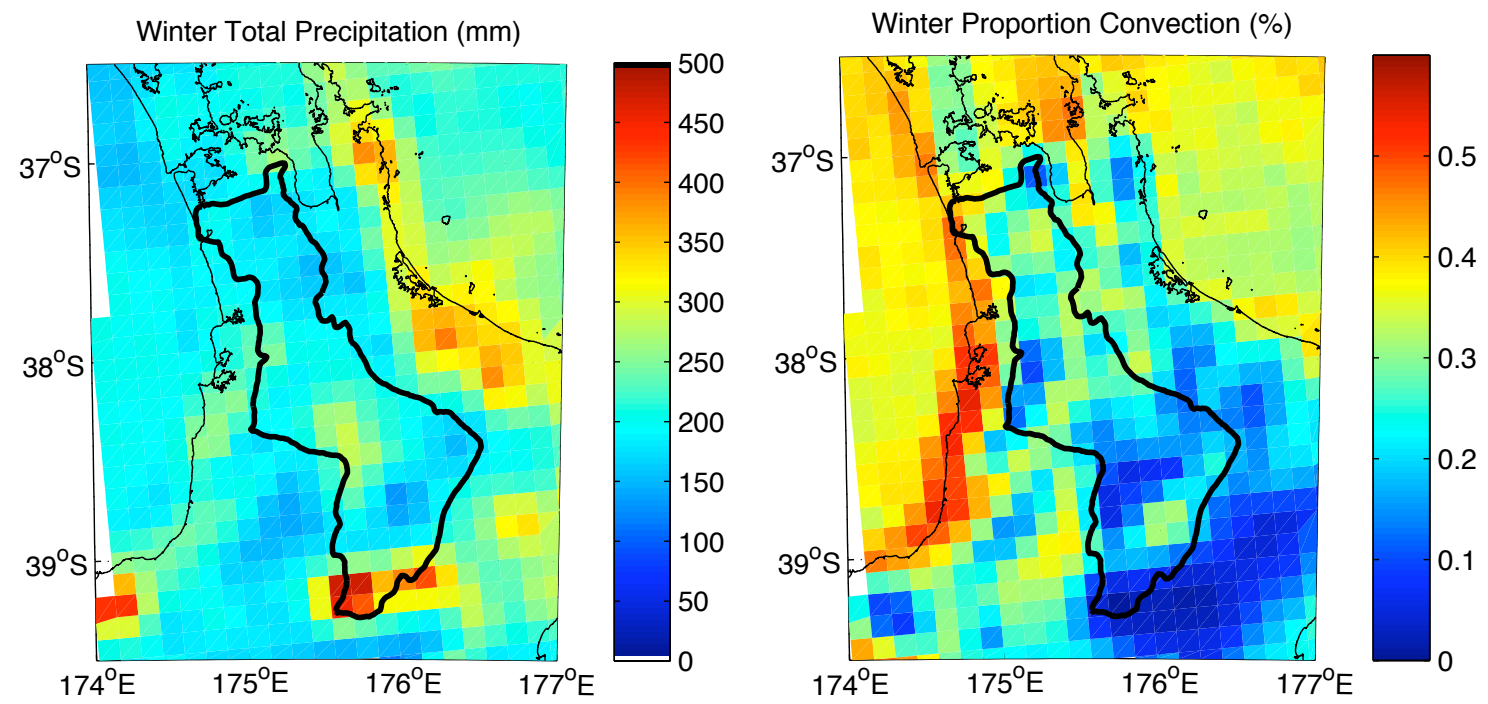

Figure 7.19 (a) Average total winter precipitation in the Waikato according to the downscaling simulation, and (b) the proportion of the precipitation that was generated through cumulus parameterisation.

also changed in winter. In previous figures the model generated similar precipitation totals for both the Kaimai Ranges and the west coast, whereas in winter there is distinctly more precipitation in the Kaimai Ranges. As the proportion of convective precipitation has not changed in the Kaimai Ranges, both the convective and resolved precipitation increased during the winter months. Convective precipitation slightly decreased throughout the Waikato River catchment in winter. The additional precipitation along this range is likely due to the entrainment of warm tropical air ahead of the cold fronts that frequently pass over the region during this season as seen in the case studies in Chapter 4.

During spring (Figure 7.20) more precipitation was generated along the west coast than the Kaimai Ranges, but total precipitation decreased across the region. Convective parameterisation is still generating much of the precipitation in the sea and 

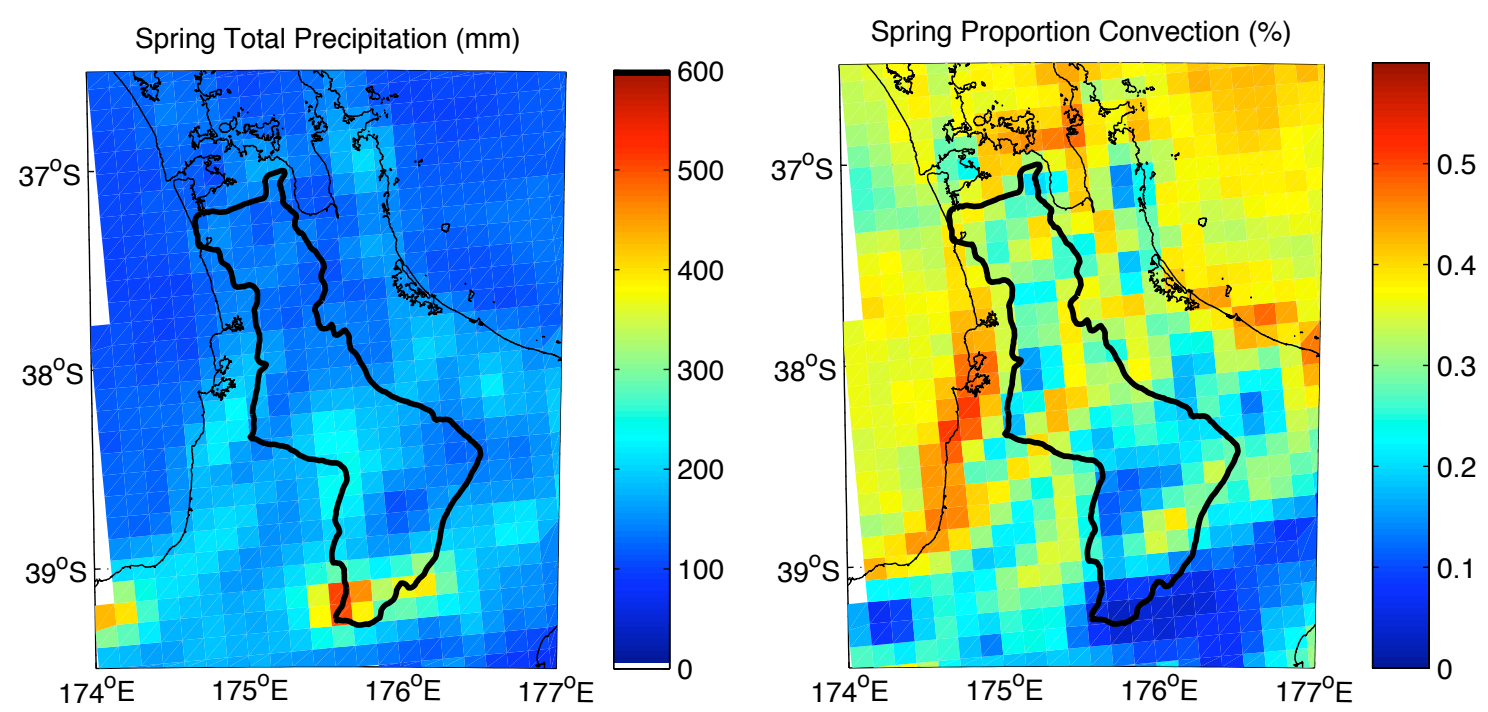

Figure 7.20 (a) Average total spring precipitation in the Waikato according to the downscaling simulation, and (b) the proportion of the precipitation that was generated through cumulus parameterisation.

along the coastlines. However, during spring there is less convective precipitation in the Waikato River catchment.

In summer, less of the precipitation is generated by cumulus parameterisation although convective precipitation is more likely to occur in the warmer air masses that are common at this time. This climatology also showed that precipitation occurs along the Kaimai Ranges more frequently in winter. This indicates that warm air is being entrained along frontal lines and is hitting New Zealand from the northeast (even if the cyclone is coming from the west). The west coast and volcanoes consistently had high precipitation levels with a small proportion of that precipitation being convective on the volcanoes.

\subsection{Subcatchment time series}

The distribution of rain gauges is uneven throughout the Waikato River catchment; in Chapter 3 an area average was used to approximate the precipitation across the catchment, but as the distribution was uneven, parts of the catchment may have been unfairly represented. The regional daily time series from Chapter 3 can be compared to 
the two-year weather simulation to show how representative the regional time series was in each subcatchment. Figure 7.21 shows that the Waikato River catchment receives

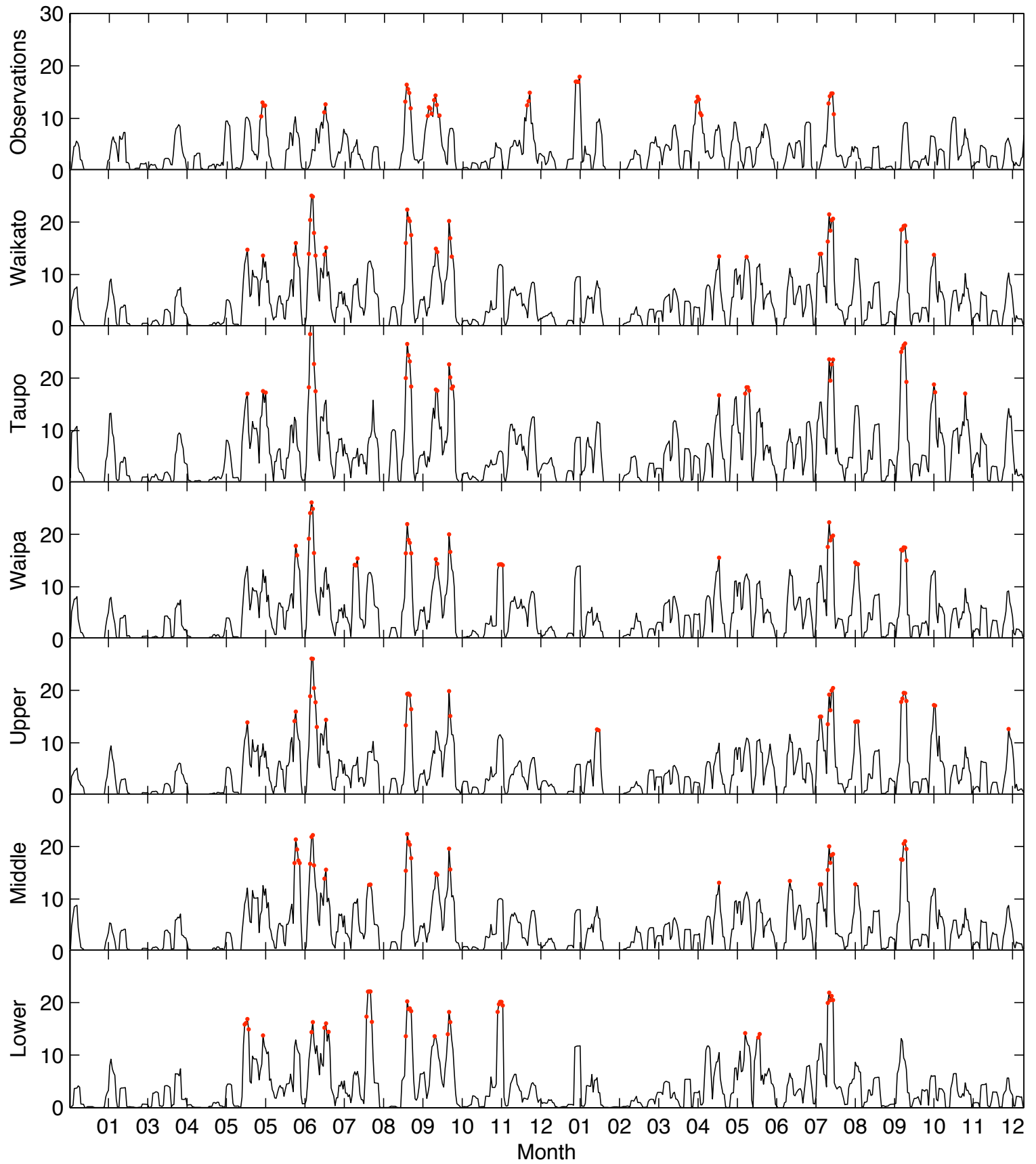

Figure 7.21 Simulated daily precipitation in each subcatchment of the Waikato River and the regionally averaged daily precipitation time series from available observation values. The red dots indicate values exceeding the 95th percentile.

larger volumes of precipitation more frequently than estimated by the regional time series based on rain gauge data alone. This was expected due to the lack of stations 
in higher altitude locations. In general the peaks occur simultaneously and therefore identify extreme events, although a percentile should have identified many of them.

Figure 7.21 also shows the time series for each of the subcatchments as defined in Figure 7.2. The heaviest precipitations are seen in the Taupo subcatchment that contains Mt Ruapehu, with the magnitude of precipitation peaks generally decreasing down river. The majority of observation gauges are located in the middle and lower subcatchments and the peaks in the regional time series are more comparable to these subcatchments. However, the peaks in each subcatchment generally line up, indicating that the heavy precipitation events affect the entire region and events affecting the Taupo and Upper catchment would still have been identified.

The downscaling simulation generates heavy precipitation more frequently than observed (Figure 7.22) as a result of fairer representation of high altitude locations in the simulation. As expected from Figure 7.21 the Taupo and Upper subcatchments, near the head of the river, have a higher occurrence of heavy precipitation days and a lower occurrence of dry days. Conversely, if the observed daily precipitation time series were gridded it would increase the occurrence of light precipitation at the expense of heavy precipitation (Ensor and Robeson, 2008) similar to the global model. This is the opposite to what is seen in the top two panels in Figure 7.22, where the model has over-predicted heavy precipitation days, possibly because of the under-simulation of precipitation over the King Country. Both of the time series (Simulated and Observed) involve assumptions and are of limited accuracy, and the real precipitation value is likely to be between the two approximations.

The daily differences between the observed time series and the simulated Waikato River catchment time series were more frequently positive indicating that generally, more precipitation was simulated than observed. The largest daily difference of $15 \mathrm{~mm}$ is only $1 \mathrm{~mm}$ below the 95th percentile threshold used to identify extremes in Chapters 3 and 4 . The daily differences are much smaller between simulated catchments.

A summary of the precipitation generated in each subcatchment from the downscal- 


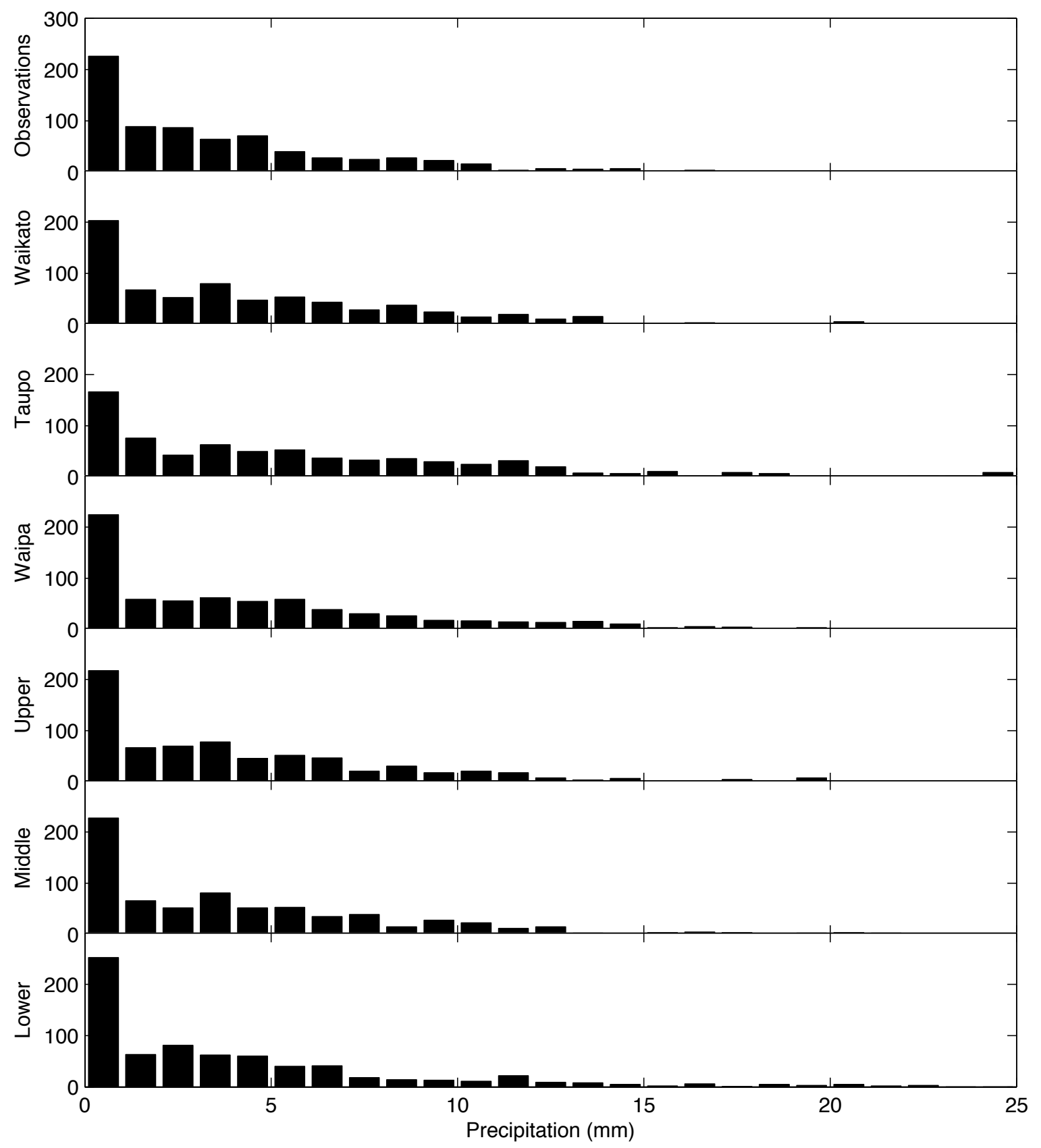

Figure 7.22 Histogram of daily precipitation values in the region and in each subcatchment of the Waikato River catchment.

ing simulation is shown in Table 7.2. For this table, the total volume of precipitation over each catchment was calculated in $\mathrm{km}^{3}$ of precipitation per year which should be proportional to the amount of water entering the river from each subcatchment. The exact volumes entering the river depend on the hydrology of the catchment, which will be determined by future researchers. This table also presents the amount of total 


\section{Table 7.2}

The total average volume of precipitation (in $\mathrm{km}^{3}$ ) in each subcatchment in each season, and the total amount of precipitation that was generated in resolved and convective model components.

\begin{tabular}{|c||c||c|c|c|c|c|}
\hline Statistic & Waikato & Taupo & Waipa & Upper & Middle & Lower \\
\hline \hline Total Annual Volume & 24.60 & 7.17 & 4.85 & 4.02 & 5.01 & 3.55 \\
\hline \hline Total Summer Volume & 3.61 & 1.18 & 0.71 & 0.60 & 0.68 & 0.44 \\
\hline Total Autumn Volume & 4.86 & 1.44 & 0.97 & 0.71 & 0.98 & 0.75 \\
\hline Total Winter Volume & 8.91 & 2.36 & 1.81 & 1.51 & 1.88 & 1.36 \\
\hline Total Spring Volume & 7.22 & 2.19 & 1.36 & 1.21 & 1.47 & 1.00 \\
\hline \hline Total Resolved & 18.45 & 5.89 & 3.48 & 3.11 & 3.48 & 2.49 \\
\hline Total convective & 6.15 & 1.28 & 1.37 & 0.91 & 1.53 & 1.07 \\
\hline
\end{tabular}

convective precipitation generated in each subcatchment per year to show the quantity of unconstrained precipitation.

In each season (and therefore over the whole year) the highest estimated precipitation volume is generated in the Taupo catchment, where Lake Taupo is used to store water for future hydroelectric generation along the Waikato River. In total, the Taupo subcatchment collects $30 \%$ of the estimated precipitation within the Waikato River catchment. Another $37 \%$ of the estimated precipitation occurs along the river in the subcatchments that feed into the power system above Lake Karapiro. The remaining estimated precipitation will enter the river system in the uncontrolled section below the power scheme.

In total, $25 \%$ of precipitation was generated through the cumulus parameterisation. This shows that only $75 \%$ of estimated precipitation can be resolved in a numerical weather model at a 12-km resolution. In the subcatchments at the head of the river this parameterisation generates $20 \%$ of the precipitation, whereas in the middle, lower and Waipa catchments it accounts for approximately $30 \%$ of the precipitation. Luckily the areas that have the highest percentage of estimated precipitation generated through parameterisation are also the areas that have lower annual totals of precipitation and less impact on the power scheme. 


\subsection{Discussion}

This chapter utilised dynamically downscaled data to provide a model climatology in data-sparse subcatchments; and to validate the mesoscale model's ability to predict precipitation over the complete range of weather systems affecting the Waikato River catchment. This data-set has no missing data or uneven gauge distribution in the region of interest. Another advantage of this downscaling simulation was that the precipitation could be split into resolved and convective precipitation. Convective precipitation represents precipitation that occurs on a sub-grid scale, the timing and location of which cannot be physically constrained by the model.

A comparison between observation data and model data interpolated to the same locations showed that the higher the resolution of the model the better the model matched the observations. Lower resolution models smoothed over areas of high precipitation on the sharp topographic features that make up New Zealand. The largest error occurred at Mt Ruapehu, where the simulation produced two to three times the quantity of precipitation. However the observation station located on Mt Ruapehu is at the Chateau, which is on the lower flanks of the volcano (the gauge is at 1,097 masl, while the volcano reaches 2,797 masl and the grid square is at $\sim 1,400$ masl) and it is expected that the precipitation levels at the top of the volcano would be higher although the tops of volcanoes (and other mountain ranges with perma-snow) are very difficult to physically gauge. These high-altitude stations had the largest mean errors and RMS errors both within the catchment and throughout New Zealand. In general the errors were small and overall the dynamic downscaling simulation is representative of the precipitation distribution within New Zealand.

The downscaling simulation differed from the high-resolution simulations, although a similar general precipitation structure existed. The model upgrade may have increased the convective precipitation generated during the downscaling simulation and merged the arrival of features involved in the January 2006 event. The timing of the cold front associated with the subtropical cyclone was delayed by four hours in the 
high-resolution simulations, and the Boundary simulation suggested that increasing the domain size could alter the timing of the front. However, the downscaling simulation had a considerably bigger domain and the delay in the arrival of the front was increased. This implies that this subtropical cyclone was poorly resolved in global models. The implication from the three simulations of this event over different domain boundaries highlights the importance of domain selection in the modelling process. However, no literature was found that investigated the importance of the boundary placement.

Unsurprisingly, more precipitation was generated during winter, and summer was driest throughout the region. Precipitation around the Kaimai Ranges was more seasonal than other areas with proportionally more precipitation during the winter months. Along the western coastline, a high proportion of the precipitation was generated through the cumulus parameterisation. In total the cumulus parameterisation accounted for approximately $25 \%$ of the precipitation in the Waikato River catchment. The implication of this is that $25 \%$ of the precipitation cannot be resolved by a numerical weather model at this resolution (which is similar to the resolution of available operational forecasts). Therefore the location where the precipitation will fall is unconstrained and cannot be relied on as in-flow into the river system.

A comparison of the regional time series used in Chapter 3 and a regional time series calculated from the downscaling simulation showed that the climate time series was representative of the Waipa, Lower and Middle subcatchments of the Waikato River, but did not represent the high precipitation values that occur over the Mt Ruapehu area. The precipitation extremes near the head of the Waikato River are larger than the extremes exhibited near the coast and in the observed time series. However, the timing of heavy precipitation is consistent and the same events are likely to be predicted as extreme in most cases, and in all of the cases where the extreme is really large (above the 98th percentile).

An implication of this dynamic downscaling is that even if the model is constrained 
by 'perfect' data (such as the FNL, an assimilation of observations) then the model cannot accurately replicate every feature of the precipitation. The model does, however, provide a good approximation to the weather conditions and the distribution of likely precipitation values. As with all weather modelling it is largely the resolution of the domain that affects how well the precipitation can be simulated. This is primarily because it affects how well the topography is represented and in a country like New Zealand where a large proportion of the precipitation pattern is influenced by topography, this is an important aspect to any modelling study. 
CHAPTER 8

\section{CONCLUSIONS}

This research investigated precipitation in the Waikato River catchment using observations and modelled data, with an additional focus on extreme precipitation events.

This was done through the statistical analysis of observational precipitation data, the use of numerical weather models, and case study analyses of weather charts and images. This chapter provides the overall summary of the thesis' aims, climate, weather forecasting, provides suggestions for further work in this area, and a final summary.

Chapter 3 investigated the precipitation climatology identifying that heavy precipitation events are uncorrelated to large-scale circulation oscillations. Additionally there was no evidence that annual precipitation totals or the magnitude and frequency of heavy precipitation events have changed since 1900. This means that the water resources within this catchment are likely to remain stable.

Chapter 4 analysed the meteorological scenarios that lead to heavy precipitation in the Waikato River catchment, identifying that the majority of heavy precipitation events coincide with the presence of a frontal band associated with a mid-latitude cyclone. Many of these cyclones undergo cyclogenesis in the Tasman Sea, particularly near the east coast of Australia.

Chapter 5 determined that the global model is skilful at predicting the occurrence of precipitation although it is not as skilful at identifying the amount or location of the precipitation. Additionally, the more frequently precipitation was predicted in subsequent model runs the more likely it was to occur. The model was more skilful 
at shorter lag times and, with a lag of less than $\sim 96$ hours, the global model provides valuable data for use as boundary constraints in a mesoscale model.

Chapter 6 investigated the WRF mesoscale model's ability to predict heavy precipitation within the Waikato River catchment. This identified that the skill of the model is dependent on the type of weather system that is approaching and is limited by the accuracy of the global model providing the boundary conditions. Multiple simulations of a single event using different model settings showed that the domain boundary had the largest impact on the generation of precipitation within the domain and that the differences between any two of the simulations was less than the difference between the control simulation and the observations, despite the control simulation providing the best simulation of the event.

Chapter 7 compared the precipitation from a continuous two-year weather simulation to daily precipitation observations around New Zealand and the Waikato. The downscaling simulation showed that the areas of higher precipitation are not well represented in the observation network. The simulation managed to generate realistic precipitation values and distributions throughout the region and was used to approximate the total volume of precipitation entering the Waikato River catchment.

\subsection{Climatology}

The climate of the Waikato River catchment is temperate, with precipitation being predominantly caused by baroclinically-driven mid-latitude cyclones. Precipitation climatologies exist in the literature, especially with the recent concerns about climate change. Most previous studies used data from a single rain gauge to represent a region. In this study we used data from multiple stations to create a regionally representative precipitation time series that approximates the volume of water entering the catchment through precipitation.

This study used three data-sets to investigate precipitation climatologies. Utilising the available data, a long-term time series was used to examine long-term trends and 
associations with large scale circulation changes, an 11-year period was used to investigate the weather associated with extreme precipitation totals, and a two-year model simulation was used to provide data in the data sparse regions of the catchment.

An analysis of the long-term precipitation climatology showed no evidence that precipitation climatology in the Waikato River catchment (specifically annual totals) has changed over the last 100 years. Large-scale circulations may affect the types and dynamics of the events causing heavy precipitation, but these are not sufficient to affect the quantity and magnitude of extreme events. Due to the sheltering effects of topography to the south, west and northeast (the directions from which cyclones approach New Zealand), the Waikato River catchment has some degree of protection from very intense storms.

Rain gauges are unevenly distributed throughout the Waikato River catchment. Dynamically downscaled data were used to determine the climatology in each of the subcatchments and approximate the total quantity of water entering the system. Downscaling is increasingly regarded as a valuable tool, especially in climate change simulations and hydrology or for testing the general skill of mesoscale models. In this case, it showed that the gauge-based regional time series likely under-estimated the precipitation values, as actual precipitation was more intense in the upper subcatchment of the river that has fewer rain gauges. Therefore the regional time series, which has few upper catchment stations represents a lower bound on precipitation volumes entering the catchment. The dynamic downscaling that had a slight tendency to over-predict may provide a better estimate of the upper bounds of precipitation volumes.

There were 99 synoptic scale weather features identified in the 63 extreme precipitation events that occurred during the 11-year period used to analyse them. The heavy precipitation in the catchment for each feature occurred under frontal bands; $98 \%$ of these frontal bands are associated with a cyclone (ex-tropical, subtropical or mid-latitude). Each cyclone developed a cold core before reaching New Zealand and had a cut-off pressure minimum in the $500 \mathrm{mb}$ geopotential height field, although the 
presence of the minima does not necessarily indicate that the precipitation will be extreme. It could be used as an indicator that the approaching cyclone may result in heavy precipitation. Cyclones are the most common cause of extreme precipitation events and are most likely to lead to flooding if the catchment is already saturated. The largest events occurred during periods of enhanced baroclinicity or when the eastward migration of a cyclone was prevented by a blocking high to the east of New Zealand. The remaining $2 \%$ of features were fronts within troughs.

Previously the ENSO has been related to the severity of ex-tropical cyclones (Sinclair, 2002), and blocking frequency (Renwick and Wallace, 1996). Pezza and Ambrizzi (2003) found that while on a hemispheric scale there was little difference in the numbers of cyclones and anticyclones, near New Zealand there was a high occurrence of anticyclone systems in La Niña years (positive MEI) and an excess of cyclonic systems in El Niño (negative MEI) years. As extreme events are uncorrelated to large-scale circulations this may mean that the weather features resulting in extremes have different causes, or are approaching from different directions. It could also mean that cyclone sequences are more common during El Niño and blocking highs and single cyclones during La Niña, although this could not be answered with the current data.

\subsection{Precipitation forecasting}

If extreme events are to be accurately forecast in the future then forecast models must be able to realistically represent the precipitation distribution caused by these events. However, it is also important that the model can represent the general weather conditions in the area to be of value as a forecasting tool.

This thesis presented the investigation into the value of NCEP numerical weather prediction models, GFS and WRF. The global data from GFS is used to initialise and constrain the WRF model, and as seen in the simulations of the January 2006 event (Chapters 6 and 7) the constraints at the boundary of the model domain can alter the development of the weather system (the timing of arrival of the fronts associated with 
the subtropical cyclone). The WRF mesoscale model can downscale the approaching synoptic features and show how they interact with the New Zealand land mass. However, errors in the GFS forecast may be propagated through to the mesoscale model, which provided motivation for including a study of the GFS forecast.

On a global scale the river catchment is small and contains very few data points in the global model used to represent this area. The abundance of dry periods in six-hour data led to a higher statistical skill score for assuming a forecast of no precipitation. Although considered to be more skilful, this has no forecasting value and the GFS forecast is more valuable when the forecasts are used together in an ensemble as a probability of precipitation forecast. The global model can predict the general synoptic scale features, and the more frequently the system appears in subsequent forecasts the more likely it is to actually occur. However, the exact timing and location of precipitation varies between model runs and precipitation totals were significantly smoothed and consistently under-predicted.

The global forecast can be used to identify the likelihood of heavily precipitating features. McBride and Ebert (2000) noted that model skill falls dramatically for heavy precipitation $(>10 \mathrm{~mm})$ and models are better at predicting the occurrence of rain than predicting the magnitude and location of peak values. Often global models predict widespread light precipitation and significant value can be added to the forecast by using a mesoscale model to determine the system's interaction with the surface. The improved topographical and land/sea boundary representation of the mesoscale model can determine how a weather system will interact with the surface and therefore generate a more realistic representation of the quantities and distribution of precipitation. However, it was found that physically unconstrained convective precipitation accounts for $\sim 25 \%$ of precipitation in this area. This is parameterised, not resolved, in operational mesoscale forecasts and can account for over $50 \%$ of precipitation during a heavy precipitation event.

The exact amount of precipitation generated was dependent on the physics pa- 
rameters of the mesoscale model (or the core during an upgrade). The Thompson microphysics (used in the Control simulations and Downscaling) generates both resolved and large-scale convective precipitation at moderate levels in both frontal and convective conditions. Without a microphysics the model did not generate scheme frontal precipitation. The other options investigated showed that Lin microphysics smoothed frontal precipitation but enhanced convection while Kessler microphysics generated more orographic enhancement and less convective precipitation. The difference between the physics schemes was less than the differences between simulations and ground observations.

To forecast precipitation in the Waikato River catchment for the purposes of estimating river flows it is recommended to use the Control simulation set up of version 2.2 of the WRF model. That is, the Thompson microphysics and the improved Kain-Fritsch cumulus parameterisation in domains with resolutions greater than 9-km. However, the domain for the model should be dependent on the direction from which the system is travelling and uncertainties, particularly from the placement of convective precipitation, should be passed through to the hydrologic models.

\subsection{Further work}

This project is part of a larger research project aimed at estimating river in-flows into the Waikato River for electricity generation purposes. Other aspects of this research are already underway, including the use of ensemble mesoscale weather predictions, and developing an appropriate hydrological model. This thesis has focused on the meteorological development and forecasting of precipitation, not examining what happens after the precipitation has reached the ground.

The climatology of extreme events over the 20th century showed that to date there have been no statistically significant trends evident in the data. Dynamical downscaling can produce representative climatologies within the Waikato River catchment that could be used with some climate change scenarios to see if the frequency and/or mag- 
nitude of extreme events are likely to change in the future or to in-fill the historical climatology.

The storm tracking system used here could be improved by including vorticity and minima in the $500 \mathrm{mb}$ geopotential height in the tracking script, and for the tracks to be conducted over a larger area. Further investigation of the possible relationship between the $500 \mathrm{mb}$ geopotential height field and the occurrence of heavy precipitation would be interesting.

It would be useful to be able to repeat validation of global forecasting data with the new GFS global ensemble data. However, the data have only been available for a short period and an archive has not been established yet. Internationally it is important to improve the representation of quickly developing systems such as sub-tropical cyclones in the data assimilation used to initiate the global models. It would also be valuable to reduce the over-prediction of the global model after model spin-up so that more accurate boundary conditions can be used to initiate mesoscale models.

With the continual development of the numerical prediction models and technology it is expected that future forecasts will improve, especially in terms of the accuracy of the input data that constrains the model. Assimilation of the new weather radar data into the models should improve the accuracy of forecasts. The new version of the WRF model has recently been released, and the new version may reduce the general over-prediction of convective precipitation and/or the under-prediction of heavy precipitation events.

As many of the heavy precipitation events contained a large proportion of convective precipitation, it would be valuable for the forecasting of precipitation into a restricted area such as a river catchment if the convective precipitation could be constrained to a specific location.

Increasing the dynamic downscaling simulation period to at least ten years would increase the statistical significance of the climatology. This data-set could be used to create finer detail model output statistics to determine the distribution of precipitation 
around the region dependent on the synoptic type and on wind direction.

Although not relevant to the aims of this thesis, the downscaled data can be used to develop a complete model climatology of New Zealand, including winds, temperatures and precipitation.

\subsection{Final summary}

The climatological analysis provided in this thesis showed that the probability of an extreme event was uncorrelated to circulation variations and that there is no evidence that the precipitation climatology has changed over the last 100 years. Seasonal forecasts may be able to predict saturation levels that will alter the probability of extreme precipitation leading to flooding but they cannot predict if extreme precipitation is likely to occur.

On a shorter time scale, numerical weather models were shown to provide valuable information out to at least a 96-hour lag. However, there is still useful information in the global forecast out to 168 hours. This study has provided useful insights into the value, reliability and shortcomings of such forecasts. The global model provides the synoptic-scale situation that can be compared to the features this research identified as having previously caused problems within the Waikato River catchment.

If a 'problematic' feature or sequence is being consistently forecast by the global model then a mesoscale model can be used to determine the quantity and distribution of precipitation from the weather system. Again, this thesis provides insight into the reliability and limitations of these forecasts. The precipitation generated in the mesoscale model can then be compared to historical values (Figure 3.8) to determine the consequences compared to previous events of similar magnitude, or fed into a hydrology model to determine river flows and flooding potential. 


\section{REFERENCES}

Alexander, L.V. and Zhang, X. and Peterson, T.C. and Caesar, J. and Gleason, B. and Klein Tank, A.M.G. and Haylock, M. and Collins, D. and Trewn, B. and Rahimzadeh, F. and Tagipour, A. and Rupa Kumar, K. and Revadekar, J. and Griffiths, G. and Vincent, L. and Stephenson, D.B. and Burn, J. and Aguilar, E. and Brunet, M. and Taylor, M. and New, M. and Zhai, P. and Rusticucci, M. and Vazquez-Aguirre, J.L. (2006), Global observed changes in daily climate extremes of temperature and precipitation, Journal of Geophysical Research 111(D05109): doi:10.1029/2005JD006290.

Anandhi, A. and Srinivas, V. and Nanjundiah, R. and Kumar, D.N. (2008), Downscaling precipitation to river basin in India for IPCC SRES scenarios using support vector machine, International Journal of Climatology 28: 401-420.

Anderson, C.J. and Arritt, R.W. and Kain, J.S. (2006), A revised version of the Kain-Fritsch Convective Parameterization and its effects in seasonl simulations, Journal of Hydrometeorology p. manuscript.

Andreoli, R.V. and Kayano, M.T. (2005), ENSO-related rainfall anomalies in South America and associated circulation features during warm and cold Pacific Decadal oscillation regimes, International Journal of Climatology 25: 20172030, DOI: 10.1002/joc.1222.

Atger, F (2001), Verification of intense precipitation forecasts from single models and ensemble prediction systems, Nonlinear processes in Geophysics 8: 401-417.

Baer, F. and Boudra, D. (1977), Numerical prediction and modification of cyclonescale precipitation, Monthly Weather Review 105: 603-617.

Barros, A.P. and Lettenmaier, D.P. (1993), Dynamic modeling of the spatial distribution of precipitation in remote mountainous areas, Monthly Weather Review 121: $1195-1214$.

Basher, R.E. and Thompson, C.S. (1996), Relationship of air temperatures in New Zealand to regional anomalies in sea-surface temperature and atmospheric circulation, International Journal of Climatology 16: 405-425.

Bradley, A.A. and Smith, J.A. (1994), The hydrometeorological environment of extreme rainstorms in the southern plains of the United States, Journal of Applied Meteorology 33(12): 1418-1431.

Brenstrum, E. (1998), The New Zealand weather book, Craig Potton Publishing.

Brier, G.W. (1950), Verification of forecasts expressed in terms of probability, Monthly Weather Review 78(1): 1-3. 
Busuioc, A. and Tomozeiu, R. and Cacciamani, C. (2008), Statistical downscaling model based on canonical correlation analysis for winter extreme precipitation events in the Emilia-Romagna region, International Journal of Climatology 28: 449-464.

Buzzi, A. and Tartaglione, N. and Malguzzi, P. (1998), Numerical simulations of the 1994 Piedmont flood: role of orography and moist processes, Monthly Weather Review 126: 2369-2383.

Carlson, T.N. (1998), Mid-latitude weather systems, The American Meteorological Society.

Chang, H-I. and Kumar, A. and Niyogi, D. and Mohanty, U.C. and Chen, F. and Dudhia, J. (2008), Impact of convection and land surface parameterizations on the simulation of the July 26, 2005 heavy rain event over Mumbai, India, Global and Planetary Change in press.

Cherubini, T. and Ghelli, A. and Lalaurette, F. (2002), Verification of precipitation forecasts over the alpine region using high-density observing network, Weather and Forecasting 17: 238-249.

Colle, B.A. (2003), Numerical simulations of the extratropical transition of Floyd (1999): structural evoluation and responsible mechanisms for the heavy rainfall over the Northeast United States, Monthly Weather Review 131: 2905-2926.

Colle, B.A. (2004), Sensitivity of orographic precipitation to changing ambient conditions and terrain geometries: an idealized modeling perspective, Journal of Atmospheric Sciences 61: 588-606.

Colle, B.A. and Mass, C.F. (2000), The 5-9 Feburary 1996 flooding event over the Pacific Northwest: sensitivity studies and evaluation of MM5 precipitation forecasts, Monthly Weather Review 128: 593-617.

Colle, B.A. and Mass, C.F. and Westrick, K. (2000), MM5 Precipitation verification over the Pacific Northwest during the 1997-99 cool seasons, Weather and Forecasting 15: 730-744.

Colle, B.A. and Olson, J.B. and Tongue, J.S. (2003), Multiseason verification of the MM5. Part II: evaluation of high-resolution precipitation forecasts over the Northeastern United States, Weather and Forecasting 18: 458-480.

Colle, B.A. and Wolfe, J.B. and Steenburgh, W.J. and Kingsmill, D.E. and Cox, J.A.W. and Shafer, J.C. (2005), High-resolution simulations and microphysical validation of an orographic precipitation event over the Wasatch mountains during IPEX IOP3, Monthly Weather Review 133: 2947-2971.

Comarazamy, D.E. and González, J.E. (2008), On the validation of the simulation of early season precipitation on the island of Puerto Rico using mesoscale atmospheric model, Journal of Hydrometeorology 9: 507-520.

Dai, A. and Fung, I.Y. and Del Genio, A. (1997), Surface observed global land precipitation variations during 1900-88, Journal of Climate 10: 2943-1962.

Dalchér, A. and Kalnay, E. and Hoffman, R.N. (1988), Medium range lagged average forecasts, Monthly Weather Review 116: 402-416. 
Daly, C. and Neilson, R.P. and Phillips, D.L. (1994), A statistical-topographic model for mapping climatological precipitation over mountainous terrain, Journal of Applied Meteorology 33: 140-158.

Day, P.C. (1926), Precipitation in the drainage area of the Great Lakes, 1874-1924, Monthly Weather Review 54(3): 85-90.

de Lisle, J.F. (1967), The climate of the Waikato basin, Earth Science Journal 1: 2-16.

Deng, A. and Stauffer, D.R. (2006), On improving 4-km mesoscale model simulations, Journal of Applied Meteorology and Climatology 45: 361-381.

Drost, F. and Renwick, J.A. and Bhaskaran, B. and Oliver, H. and McGregor, J. (2007), A simulation of New Zealand's climate during the last glacial maximum, Quaternary Science Reviews 26: 2505-2525 DOI:10.1016/j.quascirev.2007.06.005.

Du, J. and Mullen, S.L. and Sanders, F. (1997), Short-range ensemble forecasting of quantitative precipitation, Monthly Weather Review 125: 2427-2459.

Ebert, E.E. and McBride, J.L. (2000), Verification of precipitation in weather systems: determination of systematic errors, Journal of Hydrology 239: 179-202.

Ensor, L.A. and Robeson, S.M. (2008), Statistical characteristics of daily precipitation: comparisions of gridded and point datasets, Journal of Applied Meteorology and Climatology 47: 2468-2476.

Falvey, M. and Garreaud, R. (2007), Wintertime precipitation episodes in central Chile: associated meteorological conditions and orographic influences, Journal of Hydrometeorology 8: 171-193.

Friederichs, P. and Hense, A. (2008), A probabilistic forecast approach for daily precipitation totals, Weather and Forecasting 23: 659-673.

Gallus, W.A. and Baldwin, M.E. and Elmore, K.L. (2007), Evaluation of probabilistic precipitation forecasts determined from Eta and AVN forecasted amounts, Weather and Forecasting 22: 207-215 DOI:10.1175/WAF976.1.

Gallus, W.A. and Bresch, J.F. (2006), Comparision of impacts of WRF dynamic core, physics package, and initial conditions on warm season rainfall forecasts, Monthly Weather Review 134: 2632-2641.

Gangopadhyay, S. and Clark, M. and Werner, K. and Brandon, D. and Rajagopalan, B. (2004), Effects of spatial and temporal aggregation on the accuracy of statistically downscaled precipitation estimates in the upper Colorado River basin, Journal of Hydrometeorology 5: 1192-1206.

Garnier, B.J. (1950), New Zealand weather and climate, Whitcombe and Tombs Ltd.

Gershunov, A. and Cayan, D. (2003), Heavy daily precipitation frequency over the contiguous United States: sources of climate variability and seasonal predictability, Journal of Climate 16: 2752-2765.

Giorgi, F. (1990), Simulation of regional climate using a limited area model nested in a general circulation model, Journal of Climate 3: 941-963. 
Giorgi, F. and Bates, G.T. and Nieman, S.J. (1993), The multiyear surface climatology of a regional atmospheric model over the western United States, Journal of Climate 6: 75-95.

Gong, D. and Wang, S. (1999), Definition of Antartic Oscillation Index, Geophysical Research Letters 26: 459-462.

Griffiths, G.M. (2007), Changes in New Zealand daily rainfall extremes 1930-2004, Weather and Climate 27: 3-44.

Griffiths, G.M. and Salinger, M.J. and Leleu, I. (2003), Trends in extreme daily rainfall across the South Pacific and relationship to the South Pacific Convergence Zone, International Journal of Climatology 23: 847-869.

Grubišić, V. and Vellore, R.K. and Huggins, A.W. (2005), Quantitative precipitation forecasting of wintertime storms in the Sierra Nevada: senstivity to the microphysical parameterization and horizontal resolution, Monthly Weather Review 133: $2834-2859$.

Gutowski, W.J. and Decker, S.G. and Donavon, R.A. and Pan, Z. and Arritt, R.W. and Takle, E.S. (2003), Temporal-spatial scales of observed and simulated precipitation in central U.S. climate, Journal of Climate 16: 3841-3847.

Hamill, T.M. (1999), Hypothesis tests for evaluating numerical precipitation forecasts, Weather and Forecasting 14: 155-167.

Hamill, T.M. and Hagedorn, R. and Whitaker, J.S. (2008), Probabilistic forecast calibration using ECWMF and GFS ensemble reforecasts. Part II: precipitation, Monthly Weather Review 136: 2620-2632.

Hess, G.D. (1990), Numerical simulation of the August 1986 Heavy Rainfall Event in the Sydney Area, Journal of Geophysical Research 95 D3: 2073-2082.

Holton, J.R. (2004), An introduction to dynamic meteorology, 4 edn, Academic Press, Inc.

Hong, S.-Y. (1999), New global orography data sets, Office note 424, National Centers for Environmental Prediciton.

Houze, R.A. and Smull, B. F. and Dodge, P. (1990), Mesoscale organization of springtime rainstorms in Oklahoma, Monthly Weather Review 118: 613-654.

Hundecha, Y. and Bardossy, A. (2008), Statistical downscaling of extremes of daily precipitation and temperature and construction of their future scenarios, International Journal of Climatology 28: 589-610.

Ibbitt, R.P. and Henderson, R.D. and Copeland, J. and Wratt, D.S. (2001), Simulating mountain runoff with meso-scale weather model rainfall estimates: a New Zealand experience, Journal of Hydrology 239: 19-32.

IPCC (2007), Climate Change 200\%, Cambridge University Press.

Iredell, M. (2006), GFS upgrade implementation scheduled August 22, 2006 scientific decision briefing.

Iredell, M. (2007), GFS upgrade implementation scheduled May 1, 2007 scientific decision briefing. 
Jones, P.D. and Hulme, M. (1996), Calculating regional climatic time series for temperature and precipitation: methods and illustrations, International Journal of Climatology 16: 361-377.

Kain, J.S. and Fritsch, J.M. (1993), Convective parameterization for mesoscale models: The Kain-Fritcsh scheme, The representation of cumulus convection in numerical models, American Meteorological Society.

Kain, J.S. and Fritsch, M. (1990), A one-dimensional entraining/detraining plume model and its application in convective parameterization, Journal of the Atmospheric Sciences 47: 2784-2802.

Kalb, M.W. (1985), Results from a limited area mesoscale numerical simulation for 10 April 1979, Monthly Weather Review 113: 1644-1662.

Kalnay, E. and Kanamitsu, M. and Baker, W.E. (1990), Global numerical weather prediction at the National Meteorological Center, Bulletin of the American Meteorological Society 71(10): 1410-1428.

Kalnay, E. and Kanamitsu, M. and Kistler, R. and Collins, W. and Deaven, D. and Gandin, L. and Iredell, M. and Saha, S. and White, G. and Woollen, J. and Zhu, Y. and Chelliah, M. and Ebisuzaki, W. and Higgins, W. and Janowiak, J. and Mo, K.C. and Ropelewski, C. and Wang, J. and Leetmaa, A. and Reynolds, R. and Jenne, R. and Joseph, D. (1996), The NCEP/NCAR 40-year reanalysis project, Bulletin of the American Meteorological Society 77: 437-472.

Kanamitsu, M. (1989), Description of the NMC global data assimilation and forecast system, Weather and Forecasting 4: 335-342.

Kanamitsu, M. and Alpert, J.C. and Campana, K.A. and Caplan, P.M. and Deaven, D.G. and Iredell, M. and Katz, B. and Pan, H.-L. and Sela, J. and White, G.H. (1991), Recent changes implemented in to the Global Forecast System at NMC, Weather and Forecasting 6: 425-435.

Katzfey, J.J. (1995), Simulation of extreme New Zealand precipitation events. Part II: mechanisms of precipitation development, Monthly Weather Review 123: 755775 .

Kessler, E. (1969), On the distribution and continuity of water substance in atmospheric cirulation, Meteorological Monograph, Vol. 32, American Meteorological Society.

Kidson, J.W. (1994), Relationship of New Zealand daily and monthly weather patterns to synoptic weather types, International Journal of Climatology 14: 723-737.

Kidson, J.W. (2000), An analysis of New Zealand synoptic types and their use in defining weather regimes, International Journal of Climatology 20: 299-316.

Kidston, J. and Renwick, J.A. and McGregor, J. (2009), Hemispheric scale seasonality of the southern annular mode and impacts on the climate of New Zealand, Journal of Climate p. DOI:10.1175/2009JCLI2640.1.

Kincer, J.B. (1919), The seasonal distribution of precipitation and its frequency and intensity in the United States, Monthly Weather Review September: 624-631. 
Kioutsioukis, I. and Melas, D. and Zanis, P. (2008), Statistical downscaling of daily precipitation over Greece, International Journal of Climatology 28: 679-691.

Koning, A.J. and Franses, P.H. (2005), Are precipitation levels getting higher? Statistical evidence for the Netherlands, Journal of Climate 18: 4701-4714.

Konrad, C.E. (2001), The most extreme precipitation events over eastern United States from 1950-1996: considerations of scale, Journal of Hydrometeorology 2: 309-325.

Kuo, Y-H. and Cheng, L. and Bao, J-W. (1988), Numerical simulation of the 1981 Sichuan flood. Part 1: evolution of a mesoscale southwest vortex, Monthly Weather Review 116: 2481-2504.

Lenters, J.D. adn Cook, K.H. (1995), Simulation and diagnosis of the regional summertime precipitation climatology of South America, Journal of Climate 8: 29883005 .

Leslie, L.M and Holland, G. (1991), Predicting regional forecast skill using single and ensemble forecast techniques, Monthly Weather Review 119: 425-435.

Lin, Y. and Farley, R.D. and Orville, H.D. (1983), Bulk parameterization of the snow field in a cloud model, Journal of Climate and Applied Meteorology 22: 10651092.

Lo, J.C-F. and Yang, Z-L. and Pielke, R.A. (2009), Assessment of dynamical climate downscaling methodologies using the Weather Research and Forecasting (WRF) model, Journal of Geophysical Research p. manuscript.

Lovejoy, S. and Schertzer, D. (2006), Multifractuals, cloud radiances and rain, Journal of Hydrology 322: 59-88.

Lovejoy, S. and Schertzer, D. and Tsonis, A.A. (1987), Functional box-counting and multiple elliptical dimensions in rain, Science 235: 1036-1038.

Mailier, P.J. and Jolliffe, I.T. and Stephenson, D.B. (2006), Quality of weather forecasts: review and recommendations, Technical report, Royal Meteorological Society.

Manton, M.J. and Della-Marta, P.M. and Haylock, M.R. and Hennessy, K.J. and Nicholls, N. and Chamber, L.E. and Collins, D.A. and Daw, G. and Finet, A. and Gunawan, D. and Inape, K. and Isobe, H. and Kestin, T.S. and Lefale, P. and Leyu, C.H. and Lwin, T. and Maitrepierre, L. and Ouprasitwong, N. and Page, C.M. and Pahalad, J. and Plummer, N. and Salinger, M.J. and Suppiah, R. and Tran, V.L. and Trewin, B. and Tibig, I. and Yee, D. (2001), Trends in extreme daily rainfall and temperature in Southeast Asia and the South Pacific: 1961-1998, International Journal of Climatology 21: 269-284.

Mantua, N.J. and Hare, S.R. and Zhang, Y. and Walllace, J.M. and Francis, R.C. (1997), A Pacific Inderdecadal climate oscillation with impacts on Salmon Production, Bulletin of the American Meteorological Society 78: 1069-1079.

Marzban, C. and Sandgathe, S. (2006), Cluster analysis for verification of precipitation fields, Weather and Forecasting 21: 824-838. 
McBride, J.L. and Ebert, E.E. (2000), Verification of quantitative precipitation forecasts from operational numerical weather prediction models over Australia, Weather and Forecasting 15: 103-121.

McGavin, T. (Quarterly Newsletter), Notable Weather, Meteorological Society of New Zealand.

Milbrant, J.A. and Yau, M.K. (2001), A mesoscale modelling study of the 1996 Saguenay flood, Monthly Weather Review 129: 1419-1440.

Mölders, N. (2008), Suitability of the Weather Research and Forecasting (WRF) model to predict the June 2005 fire weather for interior Alaska, Weather and Forecasting 23: 953-973.

Mullan, A.B. (1995), On the linearity and stability of southern oscillation-climate relationships for New Zealand, International Journal of Climatology 15: 13651386.

Mullan, A.B. and Porteous, A. and Wratt, D.S. and Hollis, M. (2005), Changes in drought risk with climate change, Technical report, NIWA client report WLG2005-23 for Ministry for the Environment.

Munro, A.J. (1998), The Waikato regional flood event of 9-20 July 1998, The Australasian Journal of Disaster and Trama Studies 1998-2.

Munro, A.J. (2002), The Weather Bomb: 21 June 2002, Technical report, Environment Waikato.

Munro, A.J. (2004), Taupo, Waikato and Waipa management zones leap day flood event: February 29 to March 5, 2004, Technical report, Environment Waikato Regional Council.

Murphy, A.H. (1993), What is a good forecast? an essay on the natural of goodness in weather forecasting, Weather and Forecasting 8: 281-293.

Murphy, A.H. and Wilks, D.S. (1998), A case study of the use of statistical models in forecast verification: precipitation probability forecasts, Weather and Forecasting 13: 795-810.

Murphy, A.H. and Winkler, R.L. (1987), A general framework for forecast verification, Monthly Weather Review 115: 1330-1338.

Newman, M. and Compo, G. and Alexander, M. (2003), ENSO-forced variability of the Pacific decadal oscillation, Journal of Climate 16: 3853-3857.

Palecki, M.A. and Angel, J.R. and Hollinger, S.E. (2005), Storm precipitation in the United States. Part I: meteorological characteristics, Journal of Applied Meteorology 44: 933-946.

Pandey, G.R. and Cayan, D. and Dettinger, M.D. and Georgakakos, K.P. (2000), A hybrid orographic plus statistical model for downscaling daily precipitation in Northern California, Journal of Hydrometeorology 1: 491-506.

Penny, A.C. (2003), Climate Database (CLIDB) user's manual, NIWA.

Pezza, A.B. and Ambrizzi, T. (2003), Variability of southern hemisphere cyclone and anticyclone behavior: further analysis, Journal of Climate 16: 1075-1086. 
Pezza, A.B. and Simmonds, I. (2005), The first south Atlantic hurricane: unprecedented blocking, low shear and climate change, Geophysical Research Letters 32: L15712, doi:10.1029/2005GL023390.

Qian, J-H. and Seth, A. and Zebiak, S. (2003), Reinitialized versus continuous simulations for regional climate downscaling, Monthly Weather Review 131: 28572874 .

Renwick, J.A. (2004), Trends in the Southern Hemisphere polar vortex in NCEP and ECMWF reanalyses, Geophysical Research Letters pp. L07209, doi:10.1029/2003GL019302.

Renwick, J.A. and Thompson, D. (2006), The southern annular mode and New Zealand climate, Water and Atmosphere 14(2): 24-25.

Renwick, J.A. and Wallace, J.M. (1996), Relationships between north Pacific wintertime blocking, El Nino, and the PNA pattern, Monthly Weather Review pp. 2071-2076.

Ridall, G.T. (1967), Notes on the hydrology of the Waikato River, Earth Science Journal 1: 75-81.

Roper, D. (2001), Taupo/Waikato resource consents assessment of environmental effects, Technical report, Mighty River Power.

Salinger, M.J. and Basher, R.E. and Fitzharris, B.B. and Hay, J.E. and Jones, P.D. and Macveigh, J.P. and Schmidely-Leleu, I. (1995), Climate trends in the SouthWest Pacific, International Journal of Climatology 15: 285-302.

Salinger, M.J. and Griffiths, G.M. (2001), Trends in New Zealand daily temperature and rainfall extremes, International Journal of Climatology 21: 1437-1452.

Salinger, M.J. and Mullan, A.B. (1999), New Zealand climate: temperature and precipitation variations and their links with atmospheric circulation 1930-1994, International Journal of Climatology 19: 1049-1071.

Salinger, M.J. and Renwick, J.A. and Mullan, A.B. (2001), Interdecadal Pacific oscillation and South Pacific climate, International Journal of Climatology 21: 17051721, DOI 10.1002/joc.691.

Sandwell, D.T. (1987), Biharmonic spline interpolation of GEOS-3 and SEASAT altimeter data, Geophysical Research Letters 14(2): 139-142.

Schröder, S.S. (2009), Cyclones of subtropical origin in the Southwest Pacific a climatology and aspects of movement and development, $\mathrm{PhD}$ thesis, Victoria Uiversity of Wellington.

Schumacher, R.S. and Johnson, R.H. (2006), Characteristics of U.S. extreme rain events during 1999-2003, Weather and Forecasting 21: 69-85.

Selby, M.J. (1967), Erosion by high intensity rainfalls in the lower Waikato, Earth Science Journal 1: 153-156.

Seo, K.-H. and Schemm, J.-K.E. and Jones, C. and Moorthi, S. (2005), Forecast skill of the tropical intraseasonal oscillation in the NCEP GFS dynamical extended range forecasts, Climate Dynamics 25: 265-284, DOI: 10.1007/s00382-0050035-2. 
Sinclair, M.R. (1993), A diagnostic study of the extratropical precipitation resulting from Tropical Cyclone Bola, Monthly Weather Review 121: 2690-2707.

Sinclair, M.R. (1994), An objective cyclone climatology for the Southern Hemisphere, Monthly Weather Review 122: 2239-2256.

Sinclair, M.R. (1995a), A climatology of cyclogenesis for the Southern Hemisphere, Monthly Weather Review 123: 1601-1619.

Sinclair, M.R. (1995b), An extended climatology of extratropical cyclones over the Southern Hemisphere, Weather and Climate 15: 21-32.

Sinclair, M.R. (2002), How often is New Zealand hit by tropical cyclones?, Water and Atmosphere 10(1): 12-13.

Skamarock, W.C. and Klemp, J.B. and Dudhia, J. and Gill, D.O. and Barker, D.M. and Wang, W. and Powers, J.G. (2007), A description of the advanced research WRF version 2, NCAR.

Smith, J.A. and Bradley, A.A. and Baeck, M.L. (1994), The space-time structure of extreme storm rainfall in the Southern Plains, Journal of Applied Meteorology 33(12): 1402-1417.

Stauffer, D.R. and Seaman, N. (1989), Use of four-dimensional assimilation in a limited-area mesoscale model part I: experiments with synoptic-scale data, Monthly Weather Review 118: 1250-1277.

Stauffer, D.R. and Seaman, N. and Binkowski, F.S. (1990), Use of four-dimensional assimilation in a limited-area mesoscale model part II: effects of data assimilation within the planetary boundary layer, Monthly Weather Review 119: 734-754.

Sturman, A.P. and Tapper, N. (2005), The weather and climate of Australia and New Zealand, 2 edn, Oxford University Press.

Sundqvist, H. and Berge, E. and Kristjánsson, J.E. (1989), Condensation and cloud parameterization studies with a mesoscale numerical weather prediction model, Monthly Weather Review 117: 1641-1657.

Tait, A. and Henderson, R. and Turner, R. and Zheng, X. (2006), Thin plate smoothing spline interpolation of daily rainfall for New Zealand using a climatological rainfall surface, International Journal of Climatology 26: 2097-2115 doi:10.1002/joc.1350.

Thompson, G. and Rasmussen, R.M. and Manning, K. (2004), Explicit forecasts of winter precipitation using an improved bulk microphysics scheme. Part I: description and sensitivity analysis, Monthly Weather Review 132: 519-542.

Trenberth, K.E. (1991), Storm Tracks in the Southern Hemisphere, Journal of the Atmospheric Sciences 48: 2159-2178.

Trenberth, K.E. (1997), The definition of El Nino, Bulletin of the American Meteorological Society 78: 2771-2777.

Trenberth, K.E. (1999), Conceptual framework for changes of extremes of the hydrological cycle with climate change, Climatic Change 42: 327-339. 
Trenberth, K.E. and Dai, A. and Rasmussen, R.M. and Parsons, D.B. (2003), The changing character of precipitation, Bulletin of the American Meteorological Society pp. DOI: 10.1175/BAMS-84-9-1205.

Trenberth, K.E. and Mo, K.C. (1985), Blocking in the Southern Hemisphere, Monthly Weather Review 113: 3-21.

Trewin, B.C. and Trevitt, A.C.F. (1996), The development of composite temperature records, International Journal of Climatology 16: 1227-1242.

Trigo, R.M. and Palutikof, J.P. (2001), Precipitation scenarios over Iberia: a comparsion between direct GCM output and different downscaling techniques, Journal of Climate 14: 4422-4446.

Türkes, M. (1996), Spatial and temporal analysis of annual rainfall variations in Turkey, International Journal of Climatology 16: 1057-1076.

Valverde Ramirez, M. and Ferreira, N. and Fraga de Campos Velho, H. (2006), Linear and Nonlinear statistical downscaling for rainfall forecasting over southeastern Brazil, Weather and Forecasting 21: 969-989.

Wacker, U. (1995), Competition of precipitation particles in a model with parameterized cloud microphysics, Journal of Atmospheric Sciences 52(14): 2577-2589.

Widmann, M. and Bretherton, C.S. and Salathé, E.P. (2003), Statistical precipitation downscaling over the northwestern United States using numerically simulated precipitation as a predictor, Journal of Climate 16: 799-816.

Wilks, D.S. (1995), Statistical methods in atmospheric sciences, Academic Press.

Wolter, K and Timlin, M.S (1998), Measuring the strength of ENSO events: how does 1997/98 rank?, Weather 53: 315-324.

You, J. and Hubbard, K.G. and Nadarajah, S. and Kunkel, K.E. (2007), Performance of quality assurance procedures on daily precipitation, Journal of Atmospheric and Oceanic Technology 24: 821-834.

Zängl, G. (2007), Interaction between dynamics and cloud microphysics in orographic precipitation enhancement: a high-resolution modeling study of two north alpine heavy-precipitation events, Monthly Weather Review 135: 2817-2840.

Zhao, Q. and Carr, F.H. (1997), A prognostic cloud scheme for operational NWP models, Monthly Weather Review 125: 1931-1953.

Zheng, X. and Basher, R.E. and Thompson, C.S. (1997), Trend detection in regionalmean temperature series: maximum, minimum, mean, diurnal range, and SST, Journal of Climate 10(2): 317-326. 


\section{CLIMATE STATIONS}

A summary of climate stations in and around the Waikato River catchment that were used in this thesis. This provides the location of each station and the maximum time range for which data was obtained. The mean annual precipitation and mean of the monthly mean temperature (over the time period 1996 to 2005) are also included for all stations shown in Figures 3.2 and 3.3.

\begin{tabular}{|r|r|r|r|r|r|r|r|}
\hline Agent No & Latitude & Longitude & Height & Start & End & Precip. & Temp. \\
\hline 1423 & -36.86 & 174.62 & 7 & 1985 & 2007 & 1369 & 15.29 \\
\hline 1425 & -36.86 & 174.75 & 75 & 1990 & 1996 & 1888 & 15.37 \\
\hline 1427 & -36.85 & 174.77 & 49 & 1900 & 1994 & 1203 & - \\
\hline 1468 & -36.89 & 174.73 & 41 & 1949 & 2007 & 1244 & 15.36 \\
\hline 1471 & -36.93 & 174.79 & 3 & 1996 & 2000 & 1145 & - \\
\hline 1496 & -36.81 & 175.11 & 34 & 1985 & 2007 & 1288 & 15.13 \\
\hline 1500 & -37.05 & 174.54 & 237 & 1984 & 2005 & 1099 & 14.60 \\
\hline 1509 & -36.62 & 175.51 & 26 & 1996 & 2004 & 1741 & - \\
\hline 1510 & -36.67 & 175.55 & 4 & 1996 & 2007 & 1765 & - \\
\hline 1513 & -36.74 & 175.50 & 100 & 1978 & 2005 & 2016 & 15.07 \\
\hline 1516 & -36.73 & 175.69 & 10 & 1996 & 2007 & 1527 & - \\
\hline 1518 & -36.82 & 175.54 & 79 & 1996 & 2007 & 2139 & - \\
\hline 1520 & -36.83 & 175.68 & 5 & 1990 & 2007 & 1750 & 14.79 \\
\hline 1523 & -36.93 & 175.70 & 16 & 1996 & 2007 & 1884 & - \\
\hline 1524 & -37.05 & 175.52 & 1 & 1996 & 2007 & 1245 & - \\
\hline 1529 & -37.16 & 175.55 & 3 & 1946 & 2007 & 1207 & 15.07 \\
\hline 1538 & -37.24 & 175.57 & 2 & 1901 & 1987 & & - \\
\hline 1544 & -37.22 & 175.87 & 0 & 1996 & 1997 & 1287 & - \\
\hline 1545 & -37.30 & 175.55 & 9 & 1996 & 2007 & 1412 & - \\
\hline 1547 & -37.37 & 175.68 & 18 & 1990 & 2007 & 1286 & 14.73 \\
\hline 1549 & -37.34 & 175.78 & 305 & 1996 & 2001 & 1851 & - \\
\hline 1551 & -37.39 & 175.85 & 114 & 1990 & 2001 & 1628 & 14.51 \\
\hline 1554 & -37.48 & 175.67 & 16 & 1996 & 2007 & 1199 & - \\
\hline 1556 & -37.42 & 175.71 & 60 & 1996 & 2007 & 1643 & - \\
\hline 1558 & -37.42 & 175.94 & 8 & 1996 & 2000 & 1327 & - \\
\hline 1563 & -37.59 & 175.60 & 15 & 1996 & 2007 & 1073 & - \\
\hline 1564 & -37.51 & 175.65 & 12 & 1996 & 2007 & 1135 & - \\
\hline 1565 & -37.55 & 175.72 & 18 & 1907 & 1999 & 1902 & 14.44 \\
\hline & & & & & & \\
\hline
\end{tabular}




\begin{tabular}{|c|c|c|c|c|c|c|c|}
\hline Agent No & Latitude & Longitude & Height & Start & End & Precip. & Temp. \\
\hline 1567 & -37.57 & 175.86 & 132 & 1996 & 2007 & 2169 & - \\
\hline 1569 & -37.55 & 175.95 & 2 & 1980 & 2004 & 1222 & 14.55 \\
\hline 1573 & -37.66 & 175.53 & 29 & 1996 & 2007 & 1238 & - \\
\hline 1574 & -37.65 & 175.53 & 29 & 1996 & 2007 & 1090 & - \\
\hline 1579 & -37.62 & 175.80 & 55 & 1996 & 2007 & 1710 & - \\
\hline 1581 & -37.75 & 175.45 & 46 & 1996 & 2007 & 1136 & - \\
\hline 1586 & -37.78 & 175.85 & 43 & 1996 & 2007 & 1283 & - \\
\hline 1587 & -37.75 & 175.88 & 253 & 1996 & 2007 & 1806 & - \\
\hline 1589 & -37.75 & 175.97 & 392 & 1984 & 2007 & 2538 & 12.94 \\
\hline 1591 & $\begin{array}{c}-37.81 \\
\end{array}$ & 175.67 & 91 & 1996 & 2001 & 1637 & - \\
\hline 1612 & -37.67 & 176.20 & 0 & 1910 & 1996 & 1281 & - \\
\hline 1615 & -37.67 & 176.20 & 4 & 1990 & 2007 & 1203 & 14.97 \\
\hline 1617 & -37.73 & 176.00 & 255 & 1996 & 2007 & 2050 & - \\
\hline 1625 & -37.77 & 176.14 & 77 & 1996 & 2007 & 1647 & - \\
\hline 1646 & -37.82 & 176.32 & 91 & 1996 & 1996 & 1631 & 14.62 \\
\hline 1648 & -37.85 & 176.46 & 64 & 1996 & 2007 & 1401 & - \\
\hline 1651 & -37.87 & 176.66 & 63 & 1996 & 2000 & 1368 & - \\
\hline 1656 & -37.90 & 176.27 & 335 & 1996 & 2000 & 2047 & - \\
\hline 1663 & $\begin{array}{l}-37.97 \\
\end{array}$ & 176.80 & 7 & 1996 & 2007 & 1545 & - \\
\hline 1664 & -37.97 & 176.81 & 7 & 1996 & 2007 & 1402 & - \\
\hline 1666 & -37.93 & 176.87 & 3 & 1996 & 2007 & 1240 & - \\
\hline 1673 & -37.93 & 176.92 & 7 & 1990 & 2007 & 1190 & 14.24 \\
\hline 1704 & -37.96 & 177.49 & 27 & 1996 & 2002 & 1505 & - \\
\hline 1734 & -38.07 & 176.17 & 361 & 1996 & 2005 & 1742 & - \\
\hline 1742 & -38.01 & 176.27 & - & 1996 & 1996 & 1648 & - \\
\hline 1746 & -38.02 & 176.35 & 297 & 1996 & 2007 & 1951 & - \\
\hline 1751 & -38.05 & 176.55 & 405 & 1996 & 2003 & 1984 & - \\
\hline 1753 & -38.08 & 176.72 & 30 & 1954 & 1996 & 1899 & 15.78 \\
\hline 1756 & -38.04 & 176.85 & 100 & 1996 & 2007 & 1374 & - \\
\hline 1770 & -38.11 & 176.32 & 283 & 1992 & 2007 & 1330 & 12.72 \\
\hline 1774 & -38.12 & 176.81 & 19 & 1996 & 2007 & 1509 & - \\
\hline 1784 & -38.27 & 176.49 & 381 & 1996 & 2002 & 1483 & - \\
\hline 1786 & -38.30 & 176.79 & 158 & 1996 & 2007 & 1619 & - \\
\hline 1792 & -38.37 & 176.16 & 305 & 1948 & 2006 & 1258 & - \\
\hline 1797 & -38.31 & 176.42 & 435 & 1901 & 1997 & 1230 & - \\
\hline 1801 & -38.32 & 176.80 & 164 & 1996 & 2007 & 1347 & - \\
\hline 1802 & -38.35 & 176.80 & 177 & 1996 & 2007 & 1257 & - \\
\hline 1819 & -38.41 & 176.56 & 544 & 1930 & 1999 & 1303 & 8.00 \\
\hline 1824 & -38.42 & 176.79 & 189 & 1996 & 2007 & 1208 & - \\
\hline 1833 & -38.51 & 176.33 & 286 & 1996 & 2003 & 1164 & - \\
\hline 1848 & -38.64 & 176.58 & 380 & 1996 & 2005 & 1638 & - \\
\hline 1856 & -38.74 & 176.08 & 396 & 1996 & 1997 & 1615 & - \\
\hline 1857 & -38.72 & 176.07 & 351 & 1996 & 2007 & 968 & - \\
\hline
\end{tabular}




\begin{tabular}{|c|c|c|c|c|c|c|c|}
\hline Agent No & Latitude & Longitude & Height & Start & End & Precip. & Temp. \\
\hline 1858 & -38.74 & 176.08 & 400 & 1990 & 2007 & 940 & 11.82 \\
\hline 1866 & -38.03 & 177.07 & 12 & 1996 & 2007 & 1560 & - \\
\hline 1867 & -38.09 & 177.00 & 17 & 1996 & 2007 & 1571 & - \\
\hline 1874 & -38.01 & 177.29 & 5 & 1947 & 2007 & 1315 & 14.33 \\
\hline 1876 & -38.10 & 177.29 & 29 & 1996 & 1998 & 1909 & - \\
\hline 1883 & -38.15 & 177.08 & 37 & 1965 & 2002 & 1576 & 14.17 \\
\hline 1916 & -38.31 & 177.32 & 213 & 1996 & 2007 & 2056 & - \\
\hline 1945 & -36.97 & 174.78 & 2 & 1959 & 1998 & 1269 & 15.69 \\
\hline 1950 & -36.90 & 174.88 & 30 & 1996 & 2002 & 1268 & - \\
\hline 1955 & -36.90 & 174.95 & 30 & 1996 & 1999 & 1246 & - \\
\hline 1962 & -37.01 & 174.79 & 33 & 1962 & 2007 & 1075 & 15.62 \\
\hline 1965 & -37.03 & 174.96 & 23 & 1969 & 2007 & 1254 & 14.62 \\
\hline 1971 & -37.13 & 174.79 & 9 & 1996 & 1996 & 1280 & - \\
\hline 1974 & -37.26 & 174.67 & 119 & 1996 & 2007 & 1352 & - \\
\hline 2006 & -37.21 & 174.86 & 88 & 1986 & 2007 & 1153 & 14.75 \\
\hline 2009 & -37.22 & 174.92 & 59 & 1996 & 2007 & 1286 & - \\
\hline 2014 & -37.34 & 174.94 & 46 & 1996 & 2001 & 1264 & - \\
\hline 2015 & -37.35 & 174.90 & 207 & 1996 & 1999 & 1450 & - \\
\hline 2021 & -37.64 & 174.87 & 61 & 1996 & 2002 & 1311 & - \\
\hline 2027 & -37.80 & 174.87 & 7 & 1996 & 2007 & 1284 & - \\
\hline 2033 & -37.09 & 175.03 & 230 & 1996 & 2001 & 1304 & - \\
\hline 2034 & -37.09 & 175.07 & 120 & 1996 & 2003 & 1299 & - \\
\hline 2066 & -37.20 & 175.14 & 35 & 1996 & 2007 & 1222 & - \\
\hline 2068 & -37.23 & 175.36 & 2 & 2001 & 2006 & 1327 & - \\
\hline 2069 & -37.24 & 175.39 & 4 & 2002 & 2007 & 1313 & - \\
\hline 2070 & -37.20 & 175.32 & 19 & 1996 & 2007 & 1128 & - \\
\hline 2084 & -37.42 & 175.36 & 168 & 1996 & 2000 & 1608 & - \\
\hline 2090 & -37.55 & 175.35 & 23 & 1996 & 2007 & 1002 & - \\
\hline 2092 & -37.64 & 175.05 & 122 & 1996 & 2005 & 1488 & - \\
\hline 2097 & -37.70 & 175.27 & 37 & 1996 & 2006 & 1106 & - \\
\hline 2100 & -37.74 & 175.23 & 23 & 1996 & 2003 & 1180 & - \\
\hline 2101 & -37.78 & 175.31 & 40 & 1906 & 1997 & 1823 & 14.40 \\
\hline 2103 & -37.81 & 175.09 & 104 & 1952 & 2004 & 1580 & 14.47 \\
\hline 2107 & -37.89 & 175.23 & 45 & 1935 & 2006 & 1307 & - \\
\hline 2108 & -37.81 & 175.21 & 61 & 1996 & 2000 & 1347 & - \\
\hline 2111 & -37.81 & 175.30 & 34 & 1996 & 2007 & 1127 & - \\
\hline 2112 & -37.87 & 175.34 & 53 & 1990 & 2007 & 1201 & 13.85 \\
\hline 2118 & -37.88 & 175.58 & 235 & 1996 & 2007 & 1044 & - \\
\hline 2120 & -37.79 & 175.59 & 137 & 1996 & 2007 & 1231 & - \\
\hline 2122 & -37.98 & 175.47 & 61 & 1917 & 1992 & - & - \\
\hline 2125 & -37.90 & 175.47 & 44 & 1996 & 2007 & 1183 & - \\
\hline 2126 & -37.93 & 175.54 & 56 & 1948 & 2006 & 1183 & - \\
\hline 2129 & -37.96 & 175.55 & 183 & 1996 & 2007 & 1327 & - \\
\hline
\end{tabular}




\begin{tabular}{|c|c|c|c|c|c|c|c|}
\hline Agent No & Latitude & Longitude & Height & Start & End & Precip. & Temp. \\
\hline 2131 & -38.39 & 174.83 & 35 & 1905 & 2006 & 1401 & - \\
\hline 2136 & -38.17 & 174.71 & 27 & 1982 & 2007 & 1196 & 15.27 \\
\hline 2141 & -38.15 & 174.86 & 46 & 1996 & 2007 & 1640 & - \\
\hline 2145 & -38.30 & 174.72 & 5 & 1996 & 2007 & 1466 & - \\
\hline 2149 & -38.41 & 174.85 & 152 & 1996 & 2004 & 2435 & - \\
\hline 2154 & -38.53 & 174.86 & 170 & 1996 & 2007 & 2292 & - \\
\hline 2155 & -38.55 & 174.82 & 95 & 1996 & 2007 & 2561 & - \\
\hline 2160 & -38.67 & 174.67 & 30 & 1996 & 2007 & 1755 & - \\
\hline 2165 & -38.72 & 174.62 & 46 & 1969 & 1997 & 1854 & 14.36 \\
\hline 2183 & -38.08 & 175.09 & 305 & 1996 & 1999 & 1778 & - \\
\hline 2184 & -38.03 & 175.14 & 170 & 1996 & 2007 & 2378 & - \\
\hline 2187 & -38.01 & 175.34 & 37 & 1996 & 1997 & 1832 & - \\
\hline 2190 & -38.07 & 175.64 & 123 & 1923 & 1994 & - & - \\
\hline 2195 & -38.16 & 175.19 & 40 & 1996 & 2007 & 1442 & - \\
\hline 2197 & -38.09 & 175.39 & 46 & 1977 & 2007 & 1058 & 13.93 \\
\hline 2203 & -38.26 & 175.10 & 91 & 1996 & 2007 & 1844 & - \\
\hline 2204 & -38.22 & 175.23 & 37 & 1996 & 1999 & 1328 & - \\
\hline 2212 & -38.33 & 175.15 & 54 & 1959 & 2007 & 1450 & 14.03 \\
\hline 2214 & -38.34 & 175.40 & 320 & 1937 & 2006 & - & - \\
\hline 2220 & -38.40 & 175.03 & 219 & 1996 & 2007 & 1975 & - \\
\hline 2230 & -38.58 & 174.99 & 107 & 1996 & 2007 & 1602 & - \\
\hline 2233 & -38.56 & 175.36 & 488 & 1931 & 2007 & 1808 & - \\
\hline 2234 & -38.52 & 175.55 & 549 & 1947 & 1994 & - & - \\
\hline 2238 & -38.68 & 175.73 & 525 & 1996 & 1999 & 1668 & - \\
\hline 2250 & -38.89 & 175.26 & 171 & 1913 & 2007 & 1545 & 12.64 \\
\hline 2255 & -38.85 & 175.38 & 280 & 1996 & 2007 & 1329 & - \\
\hline 2260 & -38.88 & 175.73 & 427 & 1996 & 1997 & 1101 & - \\
\hline 2264 & -38.99 & 175.38 & 457 & 1996 & 2007 & 1791 & - \\
\hline 2271 & -38.89 & 176.09 & 493 & 1996 & 2005 & 1662 & - \\
\hline 2274 & -39.20 & 173.98 & 366 & 1996 & 2007 & 3221 & - \\
\hline 2283 & -39.01 & 174.18 & 30 & 1996 & 2007 & 1425 & - \\
\hline 2311 & -39.16 & 174.36 & 97 & 1996 & 2007 & 1886 & - \\
\hline 2312 & -39.15 & 174.51 & 175 & 1996 & 2007 & 2163 & - \\
\hline 2321 & -39.27 & 174.10 & 955 & 1996 & 2007 & - & - \\
\hline 2325 & -39.21 & 174.33 & 200 & 1996 & 2007 & 1959 & - \\
\hline 2333 & -39.08 & 175.20 & 219 & 1966 & 2007 & 1522 & 12.63 \\
\hline 2334 & -39.00 & 175.37 & 440 & 1996 & 2007 & 1913 & - \\
\hline 2343 & -39.02 & 175.81 & 411 & 1929 & 2006 & 1772 & - \\
\hline 2347 & -39.00 & 175.81 & 375 & 1968 & 1996 & 1645 & 12.00 \\
\hline 2351 & -39.11 & 175.09 & 125 & 1996 & 2007 & 1734 & - \\
\hline 2357 & -39.20 & 175.54 & 1097 & 1981 & 2000 & 2176 & 7.75 \\
\hline 2818 & -38.74 & 177.29 & 405 & 1996 & 2007 & 1788 & - \\
\hline 2833 & -38.81 & 177.15 & 274 & 1996 & 2005 & 1926 & - \\
\hline
\end{tabular}




\begin{tabular}{|c|c|c|c|c|c|c|c|}
\hline Agent No & Latitude & Longitude & Height & Start & End & Precip. & Temp. \\
\hline 2914 & -39.17 & 176.49 & 762 & 1996 & 2007 & 1105 & - \\
\hline 2917 & -39.17 & 176.66 & 430 & 1996 & 2007 & 1182 & - \\
\hline 2936 & -39.29 & 176.54 & 564 & 1996 & 2007 & 1593 & - \\
\hline 2937 & -39.12 & 176.51 & 455 & 1996 & 2007 & 1151 & - \\
\hline 2938 & -39.27 & 176.68 & 368 & 1996 & 1998 & 1585 & - \\
\hline 2946 & -39.23 & 176.88 & 201 & 1900 & 2004 & 1712 & - \\
\hline 2947 & -39.27 & 176.87 & 430 & 1996 & 2007 & 1466 & - \\
\hline 2967 & -39.45 & 176.49 & 351 & 1996 & 2007 & 1107 & - \\
\hline 2970 & -39.43 & 176.47 & 416 & 1996 & 2005 & 1342 & - \\
\hline 2972 & -39.44 & 176.56 & 213 & 1996 & 2007 & 1332 & - \\
\hline 2973 & -39.48 & 176.64 & 131 & 1996 & 2007 & 926 & - \\
\hline 2979 & -39.41 & 176.81 & 34 & 1996 & 2007 & 960 & - \\
\hline 2980 & -39.46 & 176.86 & 3 & 1990 & 2007 & 819 & 14.13 \\
\hline 2993 & -39.55 & 176.78 & 45 & 1996 & 2007 & 876 & - \\
\hline 2997 & -39.50 & 176.91 & 2 & 1905 & 2007 & 780 & 14.75 \\
\hline 3011 & -39.65 & 176.56 & 171 & 1996 & 2007 & 756 & - \\
\hline 3013 & -39.66 & 176.72 & 38 & 1996 & 2007 & 736 & - \\
\hline 3017 & -39.65 & 176.84 & 16 & 2005 & 2007 & 1787 & - \\
\hline 3036 & -39.78 & 176.34 & 457 & 1996 & 2007 & 1333 & - \\
\hline 3037 & -39.78 & 176.48 & 244 & 1996 & 2007 & 1034 & - \\
\hline 3042 & -39.70 & 176.58 & 223 & 1996 & 2007 & 913 & - \\
\hline 3044 & -39.76 & 176.70 & 48 & 2005 & 2007 & 1780 & - \\
\hline 3056 & -39.80 & 176.23 & 480 & 1996 & 2007 & 1682 & - \\
\hline 3095 & -39.92 & 176.25 & 396 & 2005 & 2007 & 1615 & - \\
\hline 3098 & -39.91 & 176.41 & 214 & 2005 & 2007 & 1797 & - \\
\hline 3152 & -40.01 & 175.39 & 211 & 2005 & 2007 & 1865 & - \\
\hline 3154 & -40.07 & 175.38 & 158 & 2005 & 2007 & 1069 & - \\
\hline 3171 & -40.04 & 175.47 & 165 & 2005 & 2007 & 1054 & - \\
\hline 3174 & -40.05 & 175.63 & 320 & 2005 & 2007 & 1833 & - \\
\hline 3178 & -40.07 & 175.91 & 300 & 2005 & 2007 & 1916 & - \\
\hline 3189 & -40.13 & 175.42 & 114 & 2005 & 2007 & 1822 & - \\
\hline 3535 & -39.44 & 174.07 & 261 & 2005 & 2007 & 1964 & - \\
\hline 3550 & -39.59 & 174.18 & 50 & 2005 & 2007 & 1854 & - \\
\hline 3554 & -39.59 & 174.23 & 65 & 2005 & 2007 & 1867 & - \\
\hline 3595 & -39.34 & 175.23 & 323 & 1996 & 2005 & 1810 & - \\
\hline 3596 & -39.38 & 175.27 & 560 & 1996 & 2007 & 1674 & - \\
\hline 3629 & -39.46 & 175.66 & 806 & 1950 & 2007 & 1033 & 9.23 \\
\hline 3632 & -39.47 & 175.68 & 820 & 1996 & 2007 & 1071 & 9.07 \\
\hline 3652 & -39.58 & 175.87 & 503 & 1996 & 2007 & 878 & - \\
\hline 3654 & -39.53 & 175.93 & 805 & 1996 & 2007 & 944 & - \\
\hline 3670 & -39.60 & 175.85 & 488 & 1996 & 2001 & 1402 & - \\
\hline 3671 & -39.68 & 175.80 & 433 & 1972 & 2007 & 988 & 11.54 \\
\hline 3673 & -39.63 & 175.97 & 520 & 1996 & 2007 & 1008 & \\
\hline
\end{tabular}




\begin{tabular}{|c|c|c|c|c|c|c|c|}
\hline Agent No & Latitude & Longitude & Height & Start & End & Precip. & Temp. \\
\hline 3678 & -39.72 & 175.58 & 505 & 1996 & 2007 & 1066 & - \\
\hline 3685 & -39.77 & 175.80 & 457 & 1996 & 2007 & 1246 & - \\
\hline 3689 & -39.70 & 175.91 & 422 & 1996 & 1999 & 998 & - \\
\hline 3694 & -39.89 & 175.33 & 82 & 2005 & 2007 & 1823 & - \\
\hline 3700 & -39.88 & 175.66 & 290 & 2005 & 2007 & 1823 & - \\
\hline 3715 & -39.94 & 175.04 & 15 & 2005 & 2007 & 1817 & - \\
\hline 3719 & -39.96 & 175.02 & 8 & 2005 & 2007 & 1813 & - \\
\hline 3720 & -39.93 & 175.19 & 153 & 2005 & 2007 & 1837 & - \\
\hline 3731 & -39.91 & 175.42 & 118 & 2005 & 2005 & 1820 & - \\
\hline 3737 & -40.00 & 175.54 & 182 & 2005 & 2007 & 1813 & - \\
\hline 3744 & -39.97 & 175.88 & 467 & 2005 & 2007 & 1124 & - \\
\hline 3748 & -39.96 & 175.99 & 777 & 2005 & 2007 & 1948 & - \\
\hline 7450 & -37.95 & 176.99 & 5 & 1992 & 2003 & 1299 & 15.07 \\
\hline 7556 & -37.51 & 175.67 & 13 & 1996 & 2007 & 1152 & - \\
\hline 8662 & -38.99 & 175.83 & 375 & 1996 & 2007 & 1808 & - \\
\hline 9355 & -39.42 & 175.41 & 610 & 1993 & 2007 & 1365 & 10.66 \\
\hline 9925 & -39.80 & 177.08 & 5 & 1996 & 2005 & 1235 & - \\
\hline 10768 & -38.05 & 176.61 & 415 & 2005 & 2007 & 1706 & - \\
\hline 11960 & -39.10 & 174.22 & 170 & 2005 & 2007 & 1983 & - \\
\hline 12067 & -40.13 & 175.30 & 90 & 2006 & 2006 & 1281 & - \\
\hline 12325 & -36.99 & 174.87 & 18 & 1995 & 2004 & - & 15.30 \\
\hline 12326 & -36.93 & 174.80 & 5 & 1994 & 2004 & - & 15.73 \\
\hline 12327 & -36.86 & 174.63 & 35 & 1994 & 2004 & - & 15.19 \\
\hline 12328 & -36.79 & 174.74 & 20 & 1994 & 2007 & 1923 & 16.31 \\
\hline 12428 & -37.82 & 176.32 & 91 & 1996 & 2007 & - & 14.06 \\
\hline 12432 & -39.00 & 175.81 & 375 & 1996 & 2003 & - & 12.09 \\
\hline 12453 & -37.98 & 175.76 & 115 & 2005 & 2007 & 1873 & - \\
\hline 12478 & -38.37 & 175.77 & 225 & 2005 & 2007 & 1901 & - \\
\hline 12558 & -38.10 & 175.82 & 100 & 2005 & 2007 & 1629 & - \\
\hline 12616 & -37.78 & 175.31 & 40 & 1996 & 2007 & 1824 & 13.93 \\
\hline 14045 & -39.60 & 175.52 & 480 & 2005 & 2007 & 1857 & - \\
\hline 15670 & -39.11 & 176.75 & 740 & 2005 & 2006 & 1728 & - \\
\hline 15891 & -38.00 & 175.29 & 60 & 2005 & 2005 & 1631 & - \\
\hline 16410 & -39.45 & 174.11 & 190 & 2005 & 2007 & 1940 & - \\
\hline 17030 & -37.88 & 175.74 & 106 & 1999 & 2007 & 1841 & 13.23 \\
\hline 17425 & -38.75 & 174.64 & 8 & 2005 & 2007 & 1984 & - \\
\hline 17532 & -39.93 & 175.72 & 0 & 2005 & 2007 & 1821 & - \\
\hline 18040 & -38.06 & 175.47 & 140 & 2005 & 2007 & 1848 & - \\
\hline 18055 & -37.87 & 176.66 & 70 & 2005 & 2007 & 1943 & - \\
\hline 18056 & -37.96 & 176.68 & 321 & 2005 & 2007 & 2076 & - \\
\hline 18195 & -36.85 & 174.90 & 18 & 2000 & 2004 & - & 15.46 \\
\hline 18464 & -39.20 & 175.54 & 1097 & 2000 & 2007 & 2176 & 7.70 \\
\hline 18638 & -37.45 & 175.92 & 4 & 2005 & 2007 & 1976 & - \\
\hline
\end{tabular}




\begin{tabular}{|r|r|r|r|r|r|r|r|}
\hline Agent No & Latitude & Longitude & Height & Start & End & Precip. & Temp. \\
\hline 21866 & -38.04 & 176.75 & 18 & 2001 & 2001 & - & 14.31 \\
\hline 22146 & -37.94 & 175.65 & 140 & 2005 & 2007 & 1866 & - \\
\hline 22148 & -39.32 & 173.90 & 242 & 2005 & 2006 & 1916 & - \\
\hline 22164 & -36.87 & 174.77 & 81 & 2001 & 2004 & - & 15.53 \\
\hline 22166 & -36.87 & 174.63 & 25 & 2001 & 2004 & - & 15.08 \\
\hline 22167 & -36.91 & 174.89 & 15 & 2001 & 2004 & - & 15.16 \\
\hline 22178 & -39.05 & 174.81 & 190 & 2005 & 2007 & 1989 & - \\
\hline 22254 & -36.91 & 174.82 & 30 & 2001 & 2004 & - & 16.11 \\
\hline 22370 & -39.14 & 174.03 & 185 & 2005 & 2007 & 2026 & - \\
\hline 22582 & -39.46 & 174.47 & 142 & 2005 & 2007 & 1914 & - \\
\hline 22583 & -39.35 & 174.57 & 132 & 2005 & 2007 & 1940 & - \\
\hline 22719 & -36.96 & 174.78 & 5 & 2002 & 2004 & - & 14.69 \\
\hline 23872 & -39.34 & 174.30 & 300 & 2005 & 2007 & 1973 & - \\
\hline 23899 & -38.33 & 175.15 & 62 & 2003 & 2007 & 1890 & 12.96 \\
\hline 23908 & -37.72 & 175.59 & 48 & 2002 & 2007 & 1853 & 13.92 \\
\hline 24694 & -39.00 & 174.39 & 30 & 2005 & 2007 & 1903 & - \\
\hline 25040 & -38.68 & 176.06 & 418 & 2005 & 2007 & 1563 & - \\
\hline 25162 & -37.79 & 175.07 & 118 & 2003 & 2007 & 1964 & 13.67 \\
\hline 25222 & -39.61 & 174.29 & 98 & 2005 & 2007 & 1846 & - \\
\hline 25643 & -38.98 & 175.79 & 350 & 2003 & 2007 & 1883 & 10.86 \\
\hline 25726 & -38.62 & 174.72 & 403 & 2005 & 2007 & 2004 & - \\
\hline 25760 & -39.61 & 175.87 & 462 & 2005 & 2007 & 1809 & - \\
\hline 25877 & -37.49 & 175.87 & 290 & 2005 & 2007 & 2048 & - \\
\hline 25927 & -37.67 & 175.04 & 155 & 2005 & 2007 & 1947 & - \\
\hline 26108 & -37.98 & 177.08 & 2 & 2005 & 2007 & 1287 & - \\
\hline 26117 & -37.78 & 175.31 & 45 & 2005 & 2007 & 1816 & - \\
\hline 26510 & -38.56 & 175.79 & 460 & 1996 & 2007 & 1535 & - \\
\hline 26719 & -38.62 & 176.87 & 701 & 2006 & 2007 & 1728 & - \\
\hline & & & & & & & \\
\hline
\end{tabular}


APPENDIX B

STATISTICS

\section{B.1 Event definitions}

- An event is the consecutive days that exceed the 95th percentile threshold of a five-day smoothed area integrated time series.

- The frequency of occurrence is the number of events identified per year.

- The duration is the number of consecutive days exceeding the threshold for the event, in days.

- The volume is the cumulative regional time series value over the days identified in the event, in mm per event.

- The mean intensity of an event is the total precipitation divided by the duration, in $\mathrm{mm}$.

\section{B.2 Trend calculation (including confidence calculation)}

Unless otherwise stated trends in this thesis are linear trends calculated through a least squares error method. In these linear trends the time vector $\underset{\sim}{t}$ is mapped to the variable vector $\underset{\sim}{x}$ by the linear equation,

$$
\underset{\sim}{x}=\alpha \underset{\sim}{t}+\beta
$$

where the coefficients $\alpha$ and $\beta$ are the scaling factor and shift respectively. The data can be represented by a matrix $(\mathbf{M})$ where

$$
\mathbf{M}=[\underset{\sim}{1} t]
$$

and the coefficients by the vector $\underset{\sim}{p}$, where

$$
\underset{\sim}{p}=\left[\begin{array}{l}
\beta \\
\alpha
\end{array}\right]
$$

Therefore equation B.1 can be represented by

$$
\mathbf{M} p \underset{\sim}{p}=\underset{\sim}{x}+\sigma \underset{\sim}{\zeta}
$$


where $\sigma$ is the standard deviation and $\underset{\sim}{\zeta}$ is the vector of errors. Using the least squares method* and a pseudo inverse, $\mathbf{M}^{-}$.

$$
\underset{\sim}{\hat{p}}=\mathbf{M}^{-} \underset{\sim}{x} \text { where } \mathbf{M}^{-}=\left(\mathbf{M}^{\mathbf{T}} \mathbf{M}\right)^{-1} \mathbf{M}^{\mathbf{T}}
$$

Therefore the covariance is

$$
\operatorname{cov}(\underset{\sim}{\hat{p}})=\sigma^{2}\left(\mathbf{M}^{\mathbf{T}} \mathbf{M}\right)^{-1}
$$

and the variance can be calculated by

$$
\operatorname{var}(\underset{\sim}{\hat{p}})=\sigma^{2} * \operatorname{diag}\left[\left(\mathbf{M}^{\mathbf{T}} \mathbf{M}\right)^{-1}\right] .
$$

Then calculate the trend line $(\underset{\sim}{\vartheta})$ and the residual $\underset{\sim}{\varepsilon})$.

$$
\begin{aligned}
& \underset{\sim}{\vartheta}=\underset{\sim}{\mathbf{M} \hat{p}} \\
& \underset{\sim}{\varepsilon}=\underset{\sim}{x}-\underset{\sim}{\vartheta}
\end{aligned}
$$

Now calculate the standard deviation of the residuals through

$$
\hat{\sigma}=\sqrt{\frac{{\underset{\varepsilon}{\underline{\varepsilon}}}^{T} \times \underset{\sim}{\varepsilon}}{n-2},}
$$

and calculate the covariance of the trend line

$$
\operatorname{cov}(\underset{\sim}{\vartheta})=\mathbf{M} \operatorname{cov}(\underset{\sim}{\hat{p}}) \mathbf{M}^{\mathbf{T}}
$$

and finally calculate the standard deviation,

$$
\underset{\sim}{s d}=\operatorname{diag}[\operatorname{cov}(\vartheta)]^{\frac{1}{2}} \times \hat{\sigma} .
$$

\section{B.3 Cluster analysis}

Clustering is the classification of objects into different groups, or more precisely, the partitioning of a data set into subsets (clusters), so that the data in each subset (ideally) share some common trait - often proximity according to some defined distance measure.

In k-means cluster the objective is to minimize total intra-cluster variance, or, the squared error function

$$
V=\sum_{i=1}^{k} \sum_{x_{j} \in S_{i}}\left(x_{j}-\mu_{i}\right)^{2}
$$

where there are k clusters $S_{i}, i=1,2, \ldots, k$ and $\mu_{i}$ is the centroid or mean point of all the points $x_{j} \in S_{i}$.

*The^symbol refers to an estimated value. 


\section{B.4 Forecast verification}

\section{B.4.1 Definitions}

- A model run is a single continuous computer simulation, e.g. a weather forecast showing the development of coming weather.

- The initialisation time is the time corresponding to the beginning of the model run.

- The valid time is the time when the situation was, is, or will be valid in the modelled reality, i.e. the initial time plus lag time.

- The lag is the number of hours between the initialisation time and the valid time in a model run.

\section{B.4.2 Contingency tables}

Contingency tables provide a binary comparison between forecast and observational data. This is frequently a simple yes/no question such as 'did it rain?' and can be presented in the form:

Table B.1

Contingency table format.

\begin{tabular}{cc|c|c|c}
\multicolumn{4}{c}{} & \multicolumn{3}{c}{ Observed } \\
& & yes & no & Total \\
\cline { 2 - 5 } Forecast & yes & $\mathrm{a}$ & $\mathrm{b}$ & $\mathrm{a}+\mathrm{b}$ \\
\cline { 2 - 5 } & no & $\mathrm{c}$ & $\mathrm{d}$ & $\mathrm{c}+\mathrm{d}$ \\
\cline { 2 - 5 } & Total & $\mathrm{a}+\mathrm{c}$ & $\mathrm{b}+\mathrm{d}$ & $\mathrm{n}$
\end{tabular}

When there are multiple categories, only two parameters can be calculated directly. The categorical hit rate and bias.

- Categorical Hit Rate

$$
\text { Hit Rate }=\frac{\text { number of correct predictions }}{\text { total number of predictions }}
$$

- Categorical Bias (of any category)

$$
\text { Bias }=\frac{\text { number of yes forecasts }}{\text { number of yes observations }}
$$

For all other parameters the contingency table needs to be collapsed into the 2x2 contingency table, and the following statistics can be calculated. 
- Hit Rate $(\mathrm{H})$

$$
H=(a+d) / n
$$

- Threat Score (TS) or Critical Success index (CSI)

$$
T S=a /(a+b+c)
$$

- False Alarm Rate (FAR)

$$
F A R=b /(a+b)
$$

- Probability of Detection (POD)

$$
P O D=a /(a+c)
$$

- $\operatorname{Bias}(\mathrm{BS})$

$$
B S=(a+b) /(a+c)
$$

\section{B.4.3 Time series validation}

- Mean Error (ME)

$$
M E=\frac{1}{n} \sum_{i=1}^{n}[F(i)-O(i)]
$$

where $F$ is the forecast time series and $O$ is the observation time series, both of length $n$.

- Root Mean Square Error (RMSE)

$$
R M S E=\sqrt{\frac{1}{n} \sum_{i=1}^{n}[F(i)-O(i)]^{2}}
$$

- Mean Absolute Percentage Error (MAPE)

$$
M A P E(t)=\frac{1}{n} \sum_{i=1}^{n}\left[\frac{\left|F_{i}(t)-O_{i}(t)\right|}{O_{i}(t)}\right]
$$

- Skill Score (RSS)

$$
R S S(i)=\frac{R M S E_{\text {norm }}(i)-R M S E(i)}{R M S E_{\text {norm }}(i)} * 100
$$


Where $R M S E_{\text {norm }}(i)$ is the RMSE of the comparison model (when used here the comparison model is assuming the climatological mean).

- Cross Correlation (CC)

$$
C C(i)=E[F(i+1)-F(i), O(i+1)-O(i)]
$$

where $E[\cdot]$ is the expected value operator. 
APPENDIX C

\section{WRF CONTROL FILES}

A namelist.input file that described the model parameters controls each simulation using the WRF modelling system. This appendix contains the namelist.input files for the control simulation of the January 2006 event used in Chapter 6, and the last month of the dynamic downscaling simulation used in Chapter 7.

These files are provided as they are for the simulation, and interpretation of the namelist.input files is as described in Skamarock et al. (2007).

\section{January 2006 simulation namelist.input file}

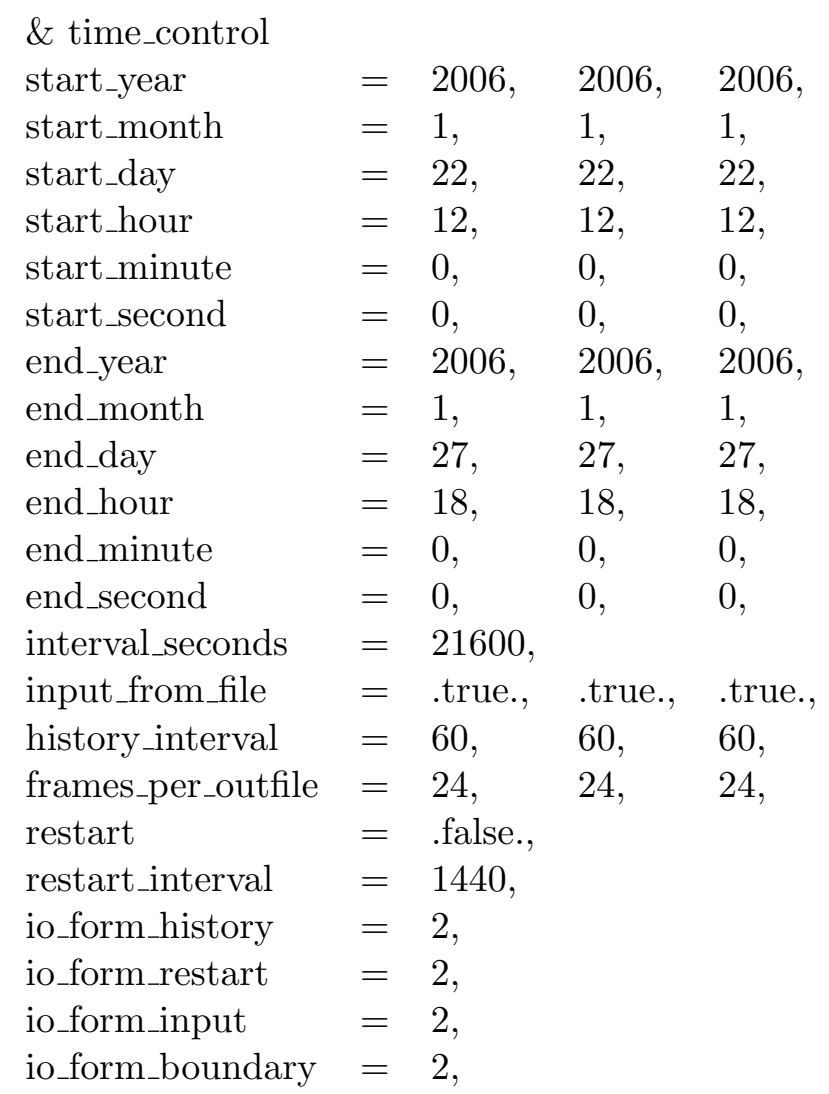




\begin{tabular}{|c|c|c|c|c|}
\hline io_form_auxinput5 & $=$ & 2 & & \\
\hline debug_level & $=$ & 0 & & \\
\hline run_days & $=$ & 0 & & \\
\hline run_hours & $=$ & 0 & & \\
\hline run_minutes & $=$ & 0 , & & \\
\hline run_seconds & $=$ & 0 & & \\
\hline / \& domains & & & & \\
\hline time_step & $=$ & 180 & & \\
\hline time_step_fract_num & $=$ & 0 & & \\
\hline time_step_fract_den & $=$ & 1 & & \\
\hline max_dom & $=$ & 3 , & & \\
\hline S_we & $=$ & 1, & 1, & 1, \\
\hline e_we & $=$ & 76 & 76 & 94, \\
\hline S_sn & $=$ & 1 & 1 & 1 \\
\hline e_sn & $=$ & 79 & 79 & 100 , \\
\hline s_vert & $=$ & 1, & 1, & 1, \\
\hline e_vert & $=$ & 28 & 28 & 28 \\
\hline$d x$ & $=$ & 27000 & 9000 & 3000, \\
\hline dy & $=$ & 27000 & 9000 & 3000 \\
\hline grid_id & $=$ & 1, & 2 & 3 \\
\hline parent_id & $=$ & 1 & 1, & 2 \\
\hline i_parent_start & $=$ & 1 & 25 & 22 , \\
\hline j_parent_start & $=$ & 1, & 27 & 23, \\
\hline parent_grid_ratio & $=$ & 1, & 3 & 3 \\
\hline parent_time_step_ratio & $=$ & 1, & 3 & 3 \\
\hline feedback & $=$ & 0 & & \\
\hline smooth_option & $=$ & 1 , & & \\
\hline \& physics & & & & \\
\hline mp_physics & $=$ & 8 & 8 & 8 \\
\hline ra_lw_physics & $=$ & 1, & 1, & 1 , \\
\hline ra_sw_physics & $=$ & 1, & 1 & 1 \\
\hline radt & $=$ & 30 & 30 & 30, \\
\hline sf_sfclay_physics & $=$ & 2 & 2 & 2 \\
\hline sf_surface_physics & $=$ & 2 & 2 & 2 \\
\hline bl_pbl_physics & $=$ & 2 & 2 & 2 , \\
\hline bldt & $=$ & 0 & 0 & 0 \\
\hline cu_physics & $=$ & 1, & 1 & 0 \\
\hline cudt & $=$ & 5 , & 5 & 5 \\
\hline isfflx & $=$ & 1 , & & \\
\hline ifsnow & $=$ & 1 , & & \\
\hline icloud & $=$ & 1, & & \\
\hline
\end{tabular}




\begin{tabular}{|c|c|c|c|c|}
\hline surface_input_source & $=$ & 1, & & \\
\hline num_soil_layers & $=$ & 4 , & & \\
\hline mp_zero_out & $=$ & 2 , & & \\
\hline maxiens & $=$ & 1 , & & \\
\hline maxens & $=$ & 3, & & \\
\hline maxens2 & $=$ & 3, & & \\
\hline maxens3 & $=$ & 16 & & \\
\hline ensdim & $=$ & 144 & & \\
\hline chem_opt & $=$ & 0 & 0 & 1, \\
\hline \& dynamics & & & & \\
\hline dyn_opt & $=$ & 2 , & & \\
\hline rk_ord & $=$ & 3, & & \\
\hline w_damping & $=$ & 0 & & \\
\hline diff_opt & $=$ & 1, & & \\
\hline km_opt & $=$ & 4 & & \\
\hline damp_opt & $=$ & 0 & & \\
\hline dampcoef & $=$ & 0.200000 & 0.200000 , & 0.200000 , \\
\hline $\begin{array}{l}\text { zdamp } \\
\text { base_temp }\end{array}$ & $\begin{array}{l}= \\
=\end{array}$ & $\begin{array}{l}5000 \\
290\end{array}$ & 5000 & \\
\hline khdif & $=$ & 750 & 750 & 750 \\
\hline kvdif & $=$ & 0 & 0 & 0 \\
\hline smdiv & $=$ & 0.100000 & 0.100000 & 0.100000 \\
\hline emdiv & $=$ & 0.010000 & 0.010000 & 0.010000 \\
\hline epssm & $=$ & 0.100000 & 0.100000 & 0.100000 \\
\hline time_step_sound & $=$ & 4 & 4 & 4 \\
\hline h_mom_adv_order & $=$ & 5 & 5 & 5 \\
\hline v_mom_adv_order & $=$ & 3 & 3 & 3 \\
\hline h_sca_adv_order & $=$ & 5 , & 5 & 5 \\
\hline v_sca_adv_order & $=$ & 3 , & 3 & 3 \\
\hline \& bdy_control & & & & \\
\hline spec_bdy_width & $=$ & 5 , & & \\
\hline spec_zone & $=$ & 1 , & & \\
\hline relax_zone & $=$ & 4 & & \\
\hline specified & $=$ & .true., & false., & false., \\
\hline periodic_x & $=$ & .false., & false., & false., \\
\hline symmetric_xs & $=$ & false., & false., & false., \\
\hline symmetric_xe & $=$ & false., & .false., & false., \\
\hline open_xs & $=$ & .false., & false., & false., \\
\hline open_xe & $=$ & false., & false., & false., \\
\hline periodic_y & $=$ & .false., & false., & false., \\
\hline symmetric_ys & $=$ & .false., & false., & false., \\
\hline
\end{tabular}




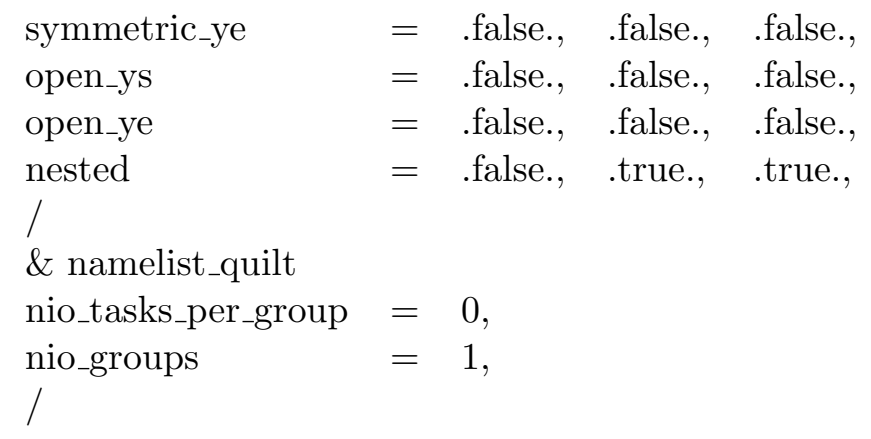

\section{Downscaling simulation namelist.input file}

This model was restarted each model month from a restart file for management of data storage. This namelist.input file is for the last month of the simulation.

\begin{tabular}{|c|c|c|c|}
\hline \multicolumn{4}{|l|}{ \& time_control } \\
\hline run_days & $=$ & 38 & \\
\hline run_hours & $=$ & 0 & \\
\hline run_minutes & $=$ & 0 & \\
\hline run_seconds & $=$ & 0 & \\
\hline start_year & $=$ & 2006 & 2006 \\
\hline start_month & $=$ & 12 & 12 \\
\hline start_day & $=$ & 01 & 01 \\
\hline start_hour & $=$ & 00 & 00 \\
\hline start_minute & $=$ & 00 & 00 \\
\hline start_second & $=$ & 00 & 00 \\
\hline end_year & $=$ & 2007 & 2007 \\
\hline end_month & $=$ & 01 & 01 \\
\hline end_day & $=$ & 06 & 06 \\
\hline end_hour & $=$ & 23 & 23 \\
\hline end_minute & $=$ & 00 & 00 \\
\hline end_second & $=$ & 00 & 00 \\
\hline interval_seconds & $=$ & 21600 & \\
\hline input_from_file & $=$ & .true., & .true., \\
\hline history_interval & $=$ & 180 & 60 \\
\hline frames_per_outfile & $=$ & 8 & 24 \\
\hline restart & $=$ & .true., & \\
\hline restart_interval & $=$ & 5760 & \\
\hline io_form_history & $=$ & 2 & \\
\hline io_form_restart & $=$ & 2 & \\
\hline io_form_input & $=$ & 2 & \\
\hline io_form_boundary & $=$ & 2 & \\
\hline debug_level & $=$ & 0 & \\
\hline
\end{tabular}




\begin{tabular}{|c|c|c|c|}
\hline \& domains & & & \\
\hline time_step & $=$ & 180 & \\
\hline time_step_fract_num & $=$ & 0 & \\
\hline time_step_fract_den & $=$ & 1 & \\
\hline max_dom & $=$ & 2 & \\
\hline S_we & $=$ & 1, & 1, \\
\hline e_we & $=$ & 100, & 115 \\
\hline S_sn & $=$ & 1 & \\
\hline e_sn & $=$ & 100 & 136 \\
\hline S_vert & $=$ & 1, & 1 \\
\hline e_vert & $=$ & 27 & 27 \\
\hline num_metgrid_levels & $=$ & 27 & \\
\hline $\mathrm{dx}$ & $=$ & 36000 & 12000 \\
\hline dy & $=$ & 36000 & 12000 \\
\hline grid_id & $=$ & 1, & 2 \\
\hline parent_id & $=$ & 1, & 1 \\
\hline i_parent_start & $=$ & 1 , & 46 \\
\hline j_parent_start & $=$ & 1, & 29 , \\
\hline parent_grid_ratio & $=$ & 1 , & 3 \\
\hline parent_time_step_ratio & $=$ & 1 , & 3 \\
\hline feedback & $=$ & 0 & \\
\hline smooth_option & $=$ & 1 & \\
\hline / & & & \\
\hline \& physics & & & \\
\hline mp_physics & $=$ & 8 & 8 \\
\hline ra_lw_physics & $=$ & 1 & 1 \\
\hline ra_sw_physics & $=$ & 1 & 1 \\
\hline radt & $=$ & 30 & 10 \\
\hline sf_sfclay_physics & $=$ & 1, & 1 \\
\hline sf_surface_physics & $=$ & 1 & 1 \\
\hline bl_pbl_physics & $=$ & 1, & 1 \\
\hline bldt & $=$ & 0 , & 0 , \\
\hline cu_physics & $=$ & 1 & 1, \\
\hline cudt & $=$ & 5 & 5 \\
\hline isfflx & $=$ & 1 & \\
\hline ifsnow & $=$ & 0 & \\
\hline icloud & $=$ & 1, & \\
\hline surface_input_source & $=$ & 1 & \\
\hline num_soil_layers & $=$ & 5 & \\
\hline ucmcall & $=$ & 0 & \\
\hline mp_zero_out & $=$ & 0 & \\
\hline maxiens & $=$ & 1, & \\
\hline maxens & $=$ & 3, & \\
\hline
\end{tabular}




\begin{tabular}{|c|c|c|c|}
\hline maxens2 & $=$ & 3 & \\
\hline maxens3 & $=$ & 16 & \\
\hline \multirow{2}{*}{\multicolumn{4}{|c|}{ / }} \\
\hline & & & \\
\hline \multicolumn{4}{|l|}{ \& fdda } \\
\hline grid_fdda & $=$ & 1 & 0 \\
\hline gfdda_inname & $=$ & "wrffdda_d01", & \\
\hline gfdda_end_h & $=$ & 168 & 168 \\
\hline gfdda_interval_m & $=$ & 360 & 360 \\
\hline fgdt & $=$ & 0 & 0 \\
\hline if_no_pbl_nudging_uv & $=$ & 0 & 0 \\
\hline if_no_pbl_nudging_t & $=$ & 1 & 1 \\
\hline if_no_pbl_nudging_q & $=$ & 1 & 1 \\
\hline if_zfac_uv & $=$ & 1, & 1 \\
\hline k_zfac_uv & $=$ & 10 & 10 \\
\hline if_zfac_t & $=$ & 0 & 0 \\
\hline k_zfac_t & $=$ & 10 & 10 \\
\hline if_zfac_q & $=$ & 0 & 0 \\
\hline k_zfac_q & $=$ & 10 & 10 \\
\hline guv & $=$ & 0.0003 & 0.0003 \\
\hline gt & $=$ & 0.0003, & 0.0003, \\
\hline gq & $=$ & 0.0003, & 0.0003, \\
\hline if_ramping & $=$ & 1, & \\
\hline dtramp_min & $=$ & 60.0 & \\
\hline io_form_gfdda & $=$ & 2 & \\
\hline \multirow{2}{*}{\multicolumn{4}{|c|}{ \& dynamics }} \\
\hline & & & \\
\hline w_damping & $=$ & 0 & \\
\hline diff_opt & $=$ & 1, & \\
\hline km_opt & $=$ & 4 & \\
\hline diff_6th_opt & $=$ & 0 & \\
\hline diff_6th_factor & $=$ & 0.12 & \\
\hline base_temp & $=$ & 290. & \\
\hline damp_opt & $=$ & 0 & \\
\hline zdamp & $=$ & 5000. & 5000., \\
\hline dampcoef & $=$ & 0.01 & 0.01 \\
\hline khdif & $=$ & 0 & 0 \\
\hline kvdif & $=$ & 0 & 0 \\
\hline non_hydrostatic & $=$ & .true., & .true., \\
\hline pd_moist & $=$ & false., & false., \\
\hline pd_scalar & $=$ & false., & .false., \\
\hline / & & & \\
\hline \& bdy_control & & & \\
\hline
\end{tabular}




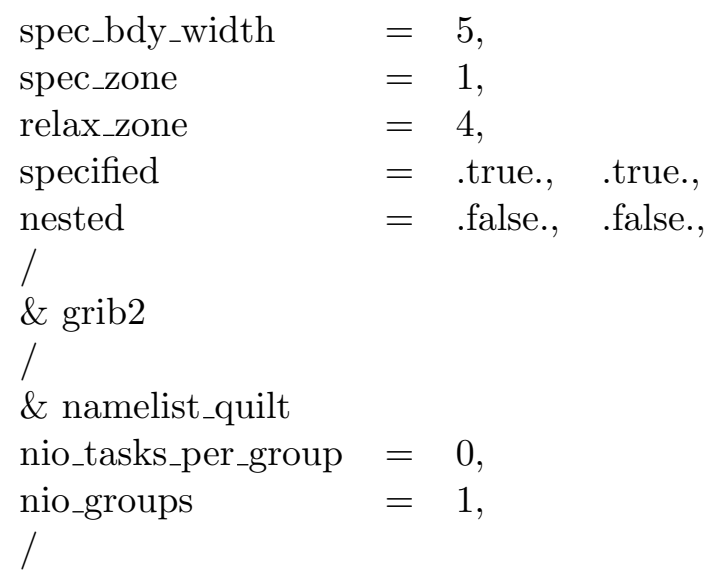

